\title{
Evaluation of the Validity of Groundwater Samples Obtained Using the Purge Water Management System at SRS
}

by

C. C. Beardsley

Westinghouse Savannah River Company

Savannah River Site

Aiken, South Carolina 29808

DOE Contract No. DE-AC09-96SR18500

This paper was prepared in connection with work done under the above contract number with the U.S.

Department of Energy. By acceptance of this paper, the publisher and/or recipient acknowledges the U.S.

Government's right to retain a nonexclusive, royalty-free license in and to any copyright covering this paper, along with the right to reproduce and to authorize others to reproduce all or part of the copyrighted paper. 
United States Department of Energy

Savannah River Site

\section{Evaluation of the Validity of Groundwater Samples Obtained Using the Purge Water Management System (PWMS) at the Savannah River Site (U)}

WSRC-TR-99-00042

Revision 0

March 1999

Westinghouse Savannah River Company/

Bechtel Savannah River Inc.

Savannah River Site

Aiken, South Carolina 29808

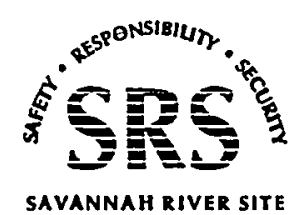


United States Department of Energy

Savannah River Site

\section{Evaluation of the Validity of Groundwater Samples Obtained Using the Purge Water Management System (PWMS) at the Savannah River Site (U)}

WSRC-TR-99-00042

Revision 0

March 1999

Westinghouse Savannah River Company/

Bechtel Savannah River Inc.

Savannah River Site

Aiken, South Carolina 29808

Prepared for the U.S. Department of Energy under Contract No. DE-AC09-96SR18500 


\section{DISCLAIMER}

This report was prepared as an account of work sponsored by an agency of the United States Government. Neither the United States Government nor any agency thereof, nor any of their employees, makes any warranty, express or implied, or assumes any legal liability or responsibility for the accuracy, completeness, or usefulness of any information, apparatus, product, or process disclosed, or represents that its use would not infringe privately owned rights. Reference herein to any specific commercial product, process, or service by trade name, trademark, manufacturer, or otherwise does not necessarily constitute or imply its endorsement, recommendation, or favoring by the United States Government or any agency thereof. The views and opinions of authors expressed herein do not necessarily state or reflect those of the United States Government or any agency thereof.

This report has been reproduced directly from the best available copy.

Available to DOE and DOE contractors from the Office of Scientific and Technical Information, P.O. Box 62, Oak Ridge, TN 37831; prices available from (615) 576-8401.

Available to the public from the National Technical Information Service, U.S. Department of Commerce; 5285 Port Royal Road, Springfield, VA 22161. 


\section{DISCLAIMER}

Portions of this document may be illegible in electronic image products. Images are produced from the best available original document. 


\section{TABLE OF CONTENTS}

LIST OF FIGURES ___ ii

LIST OF TABLES __ ii

LIST OF ACRONYMNS AND ABBREVIATIONS ___ iii

EXECUTIVE SUMMARY ___ _ 1

1.0 INTRODUCTION _ 2

2.0 METHODOLOGY _ 4

3.0 RESULTS _ 9

3.1 Comparison of Analytical Data 9

3.1.1 ABP-1A Sample Analysis Results

3.1.2 ABP-4 Sample Analysis Results__ 10

3.1.3 ARP-3 Sample Analysis Results

3.1.4 BGO-33C Sample Analysis Results _

3.2 Statistical Evaluation _ 15

3.3 Tracer Study _ 19

4.0 CONCLUSIONS _ 22

5.0 REFERENCES _ _ _ 24

APPENDIX A

APPENDIX B 


\section{LIST OF FIGURES}

Figure 1. Schematic drawing of the PWMS. 3

Figure 2. Location of wells ABP-1A, ABP-4, and ARP-3 at the Savannah River Site. 5

Figure 3. Location of well BGO-33C at the Savannah River Site. $\quad 6$

Figure 4. Plots of concentrations and activities of analytes collected at well ABP-1A by the PWMS compared to the standard collection program.

Figure 5. Plots of concentrations and activities of analytes collected at well ABP-4 by the PWMS compared to the standard collection program.

Figure 6. Plots of concentrations and activities of analytes collected at well ARP-3 by the PWMS compared to the tandard collection program.

Figure 7. Plots of concentrations and activities of analytes collected at well BGO-33C by the PWMS compared to the standard collection program.

Figure 8. Plot of tritium activities collected at well BGO-33C by the PWMS compared to the standard collection program.

Figure 9. Regression plots of concentrations and activities of analytes collected at well BGO-33C by the PWMS compared to the standard collection program.

Figure 10. Plot of bromide concentrations collected at well BGO-33C by the PWMS comparing the concentration of bromide injected into the aquifer vs. concentrations collected from the aquifer before and after injection.

Figure 11. Plot of total recoverable potassium concentrations collected at well BGO-33C comparing the concentration injected into the aquifer vs. concentrations collected historically and with the PWMS from the aquifer before and after injection.

\section{LIST OF TABLES}

Table 1. Results of screening calculations for PWMS wells.

Table 2. Schedule of sampling events at PWMS wells.

Table 3. Data codes used in the well sample analysis results.

Table 4. Frequency of sampling of SRS monitoring wells. 


\section{EXECUTIVE SUMMARY}

As part of the demonstration testing of the Purge Water Management System (PWMS) technology at the Savannah River Site (SRS), four wells were equipped with PWMS units in 1997 and a series of sampling events were conducted at each during 1997-1998. Three of the wells were located in A/M Area while the fourth was located at the Old Radioactive Waste Burial Ground in the General Separations Area.

The PWMS is a "closed-loop," non-contact, system used to collect and return purge water to the originating aquifer after a sampling event without having significantly altered the water quality. One of the primary concerns as to its applicability at SRS, and elsewhere, is whether the PWMS might resample groundwater that is returned to the aquifer during the previous sampling event.

The purpose of the present investigation was to compare groundwater chemical analysis data collected at the four test wells using the PWMS vs. historical data collected using the standard monitoring program methodology to determine if the PWMS provides representative monitoring samples.

The analysis of the groundwater chemical concentrations indicates that the PWMS sampling methodology acquired representative groundwater samples at monitoring wells $A B P-1 A, A B P-4$, ARP-3 and BGO-33C. Representative groundwater samples are achieved if the PWMS does not resample groundwater that has been purged and returned during a previous sampling event. Initial screening calculations, conducted prior to the selection of these four wells, indicated that groundwater velocities were high enough under the ambient hydraulic gradients to preclude resampling from occurring at the time intervals that were used at each well. Corroborating evidence included a tracer test that was conducted at BGO-33C, the high degree of similarity between analyte concentrations derived from the PWMS samples and those obtained from historical protocol sampling, as well as the fact that PWMS data extend all previously existing concentration trends to the present time. The latter line of evidence is considered to be the most powerful in demonstrating that representative samples are being acquired by the PWMS because it is highly unlikely that previously existing concentration trends would continue if resampling had occurred. 


\subsection{INTRODUCTION}

Standard procedure for obtaining protocol groundwater monitoring samples at the Savannah River Site (SRS) calls for extracting or "purging" sufficient quantities of groundwater to allow removal of stagnant water and to allow certain key indicator parameters to stabilize prior to collection of samples. The water extracted from a well prior to sample collection is termed "purge water" and must be managed in an approved fashion if it contains hazardous and/or radiological constituents that exceed specified healthbased limits described in the Investigation Derived Waste Management Plan (WSRC, 1994). Typical management practices include containerization, transportation, treatment, and disposal via Clean Water Act-permitted facilities.

A technology for handling purge water that eliminates the need to containerize and transport this water to a disposal facility has been developed. This technology, termed the Purge Water Management System (PWMS), is currently under pilot stage deployment at SRS. The PWMS is a "closed-loop," non-contact system used to collect and return purge water to the originating aquifer after a sampling event without significantly altering the water quality. A schematic drawing of the PWMS is in Figure 1. The system has been successfully demonstrated at both a "clean" well, P-26D, and a "contaminated" well, MCB-5, by comparing chemical concentration data obtained by PWMS sampling against the historical data record for each of these wells (Hiergesell et al., 1996). In both cases the PWMS was found to yield sample results that were indistinguishable from the results of the historical protocol sampling conducted at those same wells.

For any method used to sample groundwater, sample representativeness is a primary concern. Suitability of the PWMS to collect representative monitoring samples, while allowing for the extraction and return of large volumes of purge water back to the aquifer, is the chief issue to address for the successful demonstration of the technology. The purpose of the present investigation was to evaluate the results of groundwater sampling using PWMS units deployed at four additional, "typical" SRS wells monitoring groundwater contamination. If the groundwater samples collected using the PWMS system can be demonstrated to be representative of the groundwater flow system, then the samples are considered valid. 


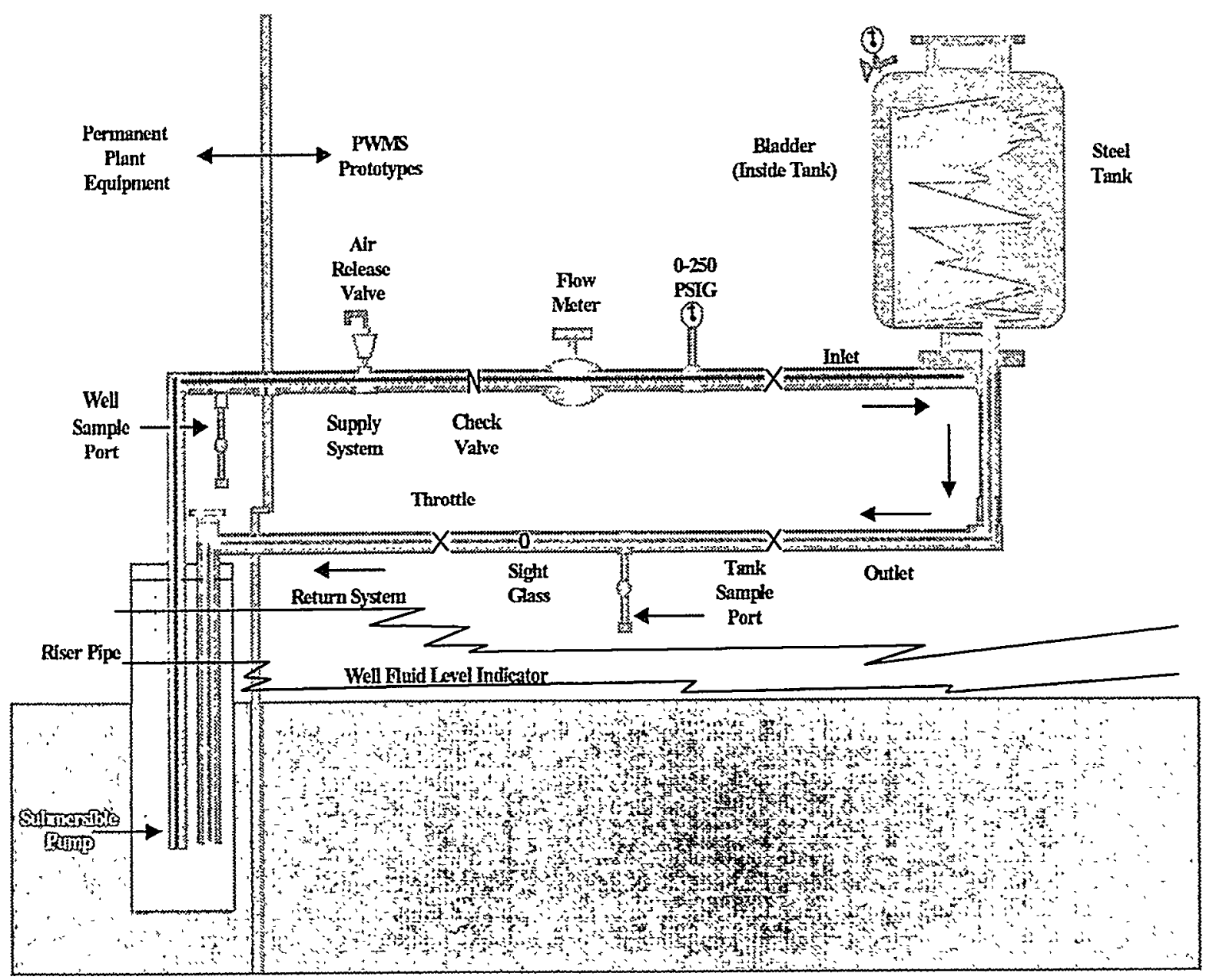

Figure 1. Schematic drawing of the purge water management system. 


\subsection{METHODOLOGY}

Three wells in the A/M Area and one well in the General Separations Area (GSA) were selected to demonstrate use of the PWMS (Figures 2 and 3):

ABP-1A Located at the A-Area Metals Burning Pit, A/M Area

ABP-4 Located at the A-Area Metals Burning Pit, A/M Area

ARP-3 Located at the A-Area Burning/Rubble Pits, A/M Area

BGO-33C Located at the Old Radioactive Waste Burial Ground, GSA

Initially, these wells were evaluated using a screening method to determine whether they were good candidates for the PWMS technology. The first part of the screening method was to determine the size of the aquifer volume from which groundwater originates during typical sampling events at each well. This volume is later refilled when water is returned from the PWMS tank. The geometric shape of the aquifer volume is assumed to be a cylinder having its axis coincident with the borehole axis and a height equal to the well screen length. Using an assumed porosity and a purged volume based upon well records, the radius of the cylindrical volume can be calculated.

The second part of the screening involved calculating how far the groundwater in the cylinder migrates between sampling events at each of the wells. Theoretically, groundwater must migrate at least twice the length of the radius of the cylinder to avoid being drawn back into the well during a second sample event. The groundwater velocity near each well screen was determined and the distance traveled during the period between sample events calculated. It was assumed that sampling events would be separated by a minimum 90-day period.

Suitability was determined by evaluating whether the pore water in the cylinder migrated far enough between 90-day sampling events to prevent groundwater from being resampled during the second sampling event. All four wells evaluated in this investigation were determined to have natural groundwater velocities sufficiently high to prevent resampling from occurring, including BGO-33C, the well with the lowest hydraulic conductivity. Specific data from the screening calculations for each well are presented in Table 1. 


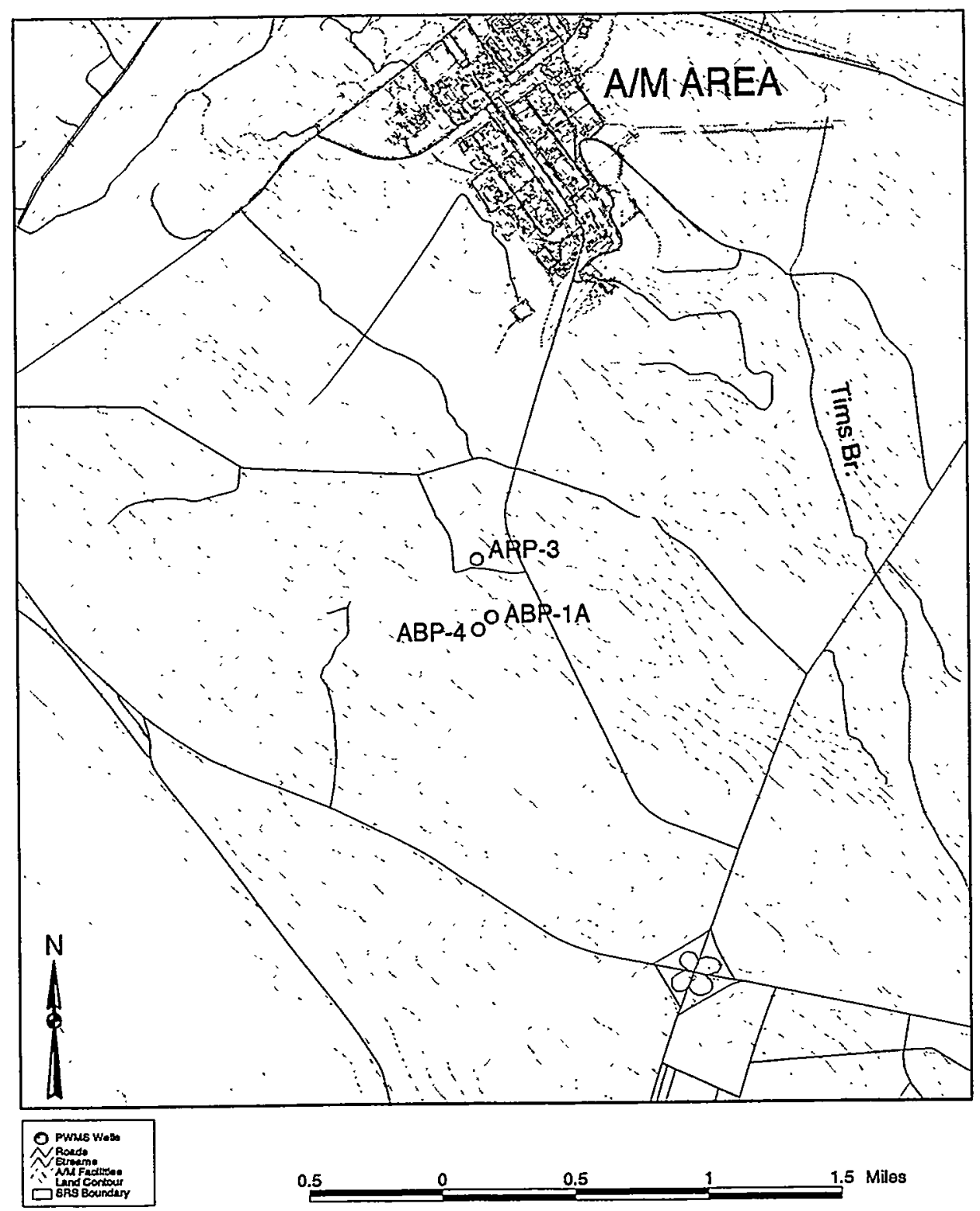

Figure 2. Location of wells ABP-1A, ABP-4, and ARP-3 at the Savannah River Site. 


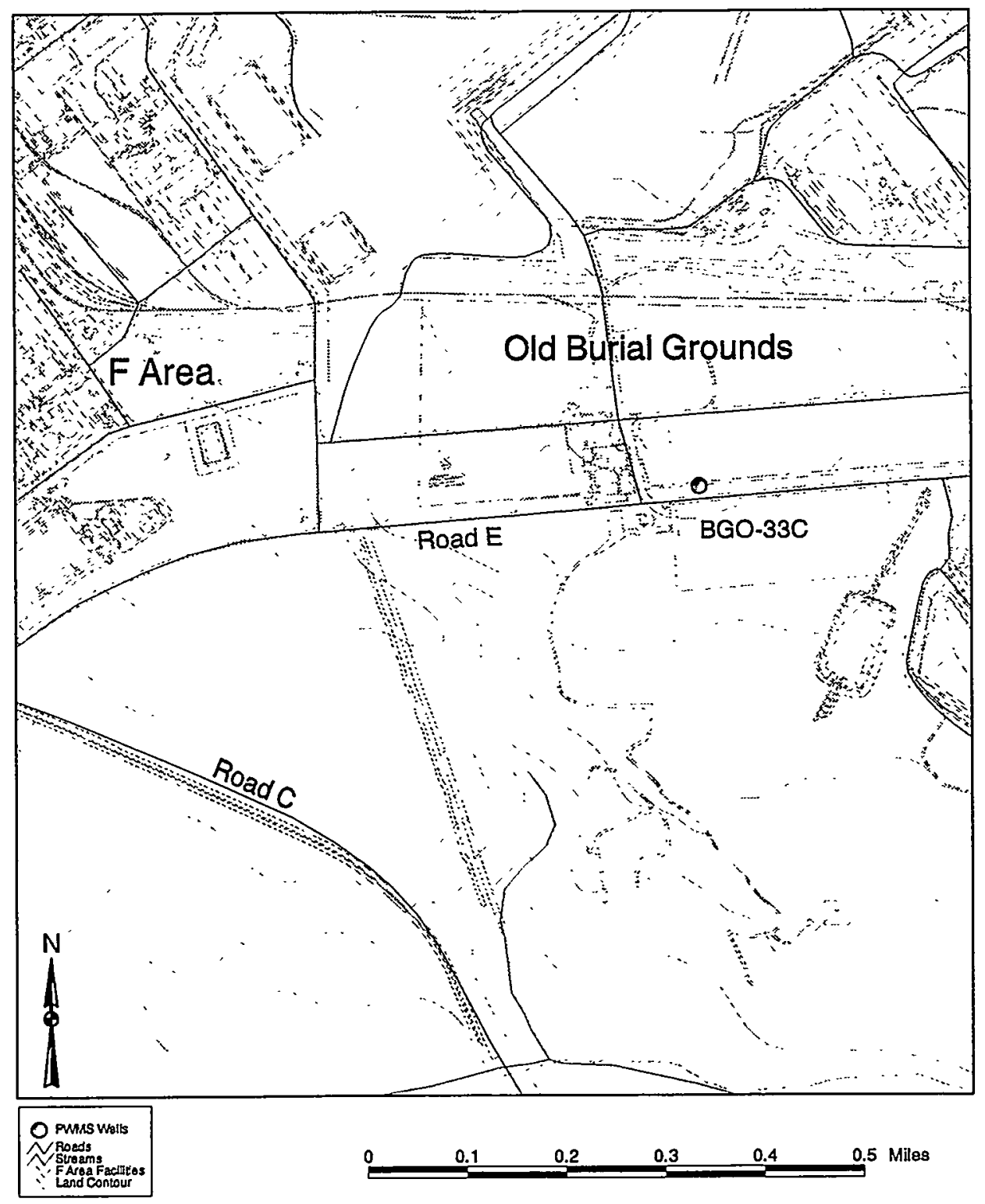

Figure 3. Location of well BGO-33C at the Savannah River Site. 
Table 1. Results of screening calculations for PWMS wells.

\begin{tabular}{|c|c|c|c|}
\hline Well & $\begin{array}{c}\text { Aquifer Material Cylinder } \\
\text { Radius (ft) }\end{array}$ & $\begin{array}{c}\text { Minimum 90-Day } \\
\text { Groundwater Migration } \\
\text { Distance (ft) }\end{array}$ & $\begin{array}{c}\text { Calculated 90-Day } \\
\text { Groundwater Migration } \\
\text { Distance (ft) }\end{array}$ \\
\hline ABP-1A & 0.87 & 1.73 & 18 \\
\hline ABP-4 & 0.74 & 1.49 & 18 \\
\hline ARP-3 & 0.65 & 1.30 & 18 \\
\hline BGO-33C & 1.90 & 3.80 & 7.4 \\
\hline
\end{tabular}

The sampling schedule.followed for evaluation of the use of the PWMS at the four wells is presented in Table 2.

Table 2. Schedule of sampling events at PWMS wells.

\begin{tabular}{|c|c|c|c|}
\hline Well & Sampling Event No. 1 & Sampling Event No. 2 & Sampling Event No. 3 \\
\hline ABP-1A & Nov. 11, 1997 & Aug. 13, 1998 & - \\
\hline ABP-4 & Nov. 11, 1997 & Sept. 28, 1998 & - \\
\hline ARP-3 & Nov. 12, 1997 & Aug. 13, 1998 & - \\
\hline BGO-33C & Dec. 16, 1997 & June 15, 1998 & Dec. 17, 1998 \\
\hline
\end{tabular}

The chemical analysis data from these sample collections were compared with analyte concentration data from protocol samples collected previously at the same wells. The historical data were obtained as part of the routine SRS groundwater-monitoring program.

The analytes selected for comparison of the PWMS sampling with the historical collections included naturally occurring analytes as well as chemical and radiological contaminants known to exist in the vicinity of the wells. The analytes, which were also evaluated in the previous study (Hiergesell et al., 1996), included:

$\mathrm{pH}$

Specific conductance

Calcium, total recoverable

Potassium, total recoverable

Sodium, total recoverable
Chromium, total recoverable

Iron, total recoverable

Manganese, total recoverable

Aluminum, total recoverable

Lead, total recoverable
Chloride

Carbon tetrachloride

Tetrachloroethylene

Trichloroethylene

Gross alpha

For BGO-33C, tritium concentrations in the PWMS-collected samples and in the historical samples were also compared. And, because of the lower hydraulic conductivity at BGO-33C, a tracer study was 
conducted at that well. Potassium bromide $(\mathrm{KBr})$ was injected into the aquifer on the second PWMS sampling event (June 15, 1998) at BGO-33C, after a "background" sample was first collected from the aquifer. Then, 180 days after the potassium bromide injection, the aquifer was sampled again using the PWMS unit to determine if any of the tracer remained.

Sampling of each of the four wells installed with a PWMS unit was identical and followed SRS standard groundwater sampling procedure (WSRC, 1992). Accordingly, groundwater was pumped at a flow rate of approximately one gallon per minute (gpm) into the PWMS unit bladder tank (Figure 1). After a minimum of two well volumes of purge water was pumped into the tank, the field indicator parameters, $\mathrm{pH}$, water temperature, specific conductance, and turbidity, were monitored at the well sampling port to determine the point at which their levels stabilized (i.e., when the measurements for each parameter varied by no more than ten percent of each other). Once stabilization occurred, a groundwater sample was collected using the PWMS at a location ahead or "influent" to the PWMS tank while tank filling continued. This sample is, thus, considered to be a "snapshot" of the groundwater unaffected by stagnant well conditions that might have occurred between sampling events.

Samples were shipped to an off-site analytical laboratory under contract to SRS for the performance of analytical services related to the ongoing groundwater monitoring program. A rigorous laboratory quality assurance process is a major component of this program. Once chemical analysis results were returned from the laboratory, the field and analytical data were validated.

Data processing for the comparison of the PWMS and historical sampling involved downloading the relevant data for each well from the SRS Geochemical Information Management System (GIMS) database to facilitate the comparison of historical and PWMS sample analyses. Scatter diagrams were constructed for each of the analytes selected to make the comparison for each of the wells. The data from each well were then examined to determine: 1) if PWMS samples fall within historical ranges and 2) if any discernible historical trends could be identified in the historical record and whether PWMS samples reflect a continuation of concentration trends. A statistical evaluation was made of the two data sets for each analyte to confirm comparisons made using the scatter diagrams. 


\subsection{RESULTS}

The results of the components of the comparison study, including comparison of analytical data by well, the statistical evaluation, and the tracer study, are presented below.

\subsection{Comparison of Analytical Data}

The results of the scatter diagram comparison of the PWMS sample analyses with the record of historical analyses are presented by well in Figures 4 to 7 . The data used in the comparison for each well are provided in Appendix A. The results are discussed below for each well.

Data codes used in the figures and in the data presented in Appendix A are identified in Table 3.

Table 3. Data codes used in the well sample analysis results.

\begin{tabular}{|c|c|}
\hline \multicolumn{2}{|c|}{ Analyte Codes } \\
\hline Code & Analyte \\
\hline FPH & Field pH \\
\hline CAND & Specific conductance \\
\hline KTOT & Calcium, total recoverable \\
\hline NATOT & Potassium, total recoverable \\
\hline CRTOT & Sodium, total recoverable \\
\hline FETOT & Chromium, total recoverable \\
\hline MNTOT & Iron, total recoverable \\
\hline ALTOT & Manganese, total recoverable \\
\hline PBTOT & Aluminum, total recoverable \\
\hline CL & Lead, total recoverable \\
\hline CCLA & Chloride \\
\hline TCLEE & Carbon tetrachloride \\
\hline TRCLE & Tetrachloroethylene \\
\hline ALPHAG & Trichloroethylene \\
\hline TRITU & Gross alpha \\
\hline BR & Tritium \\
\hline Analytical Result Qualifier Codes \\
\hline Code & Qualification \\
\hline$\#$ & Result has no qualifier - interpret as reported \\
\hline J & Result is estimated quantity \\
\hline U, UI & Result is less than sample quantitation limit \\
\hline
\end{tabular}




\subsubsection{ABP-1A Sample Analysis Results (Figure 4)}

Among the potential contaminants for which PWMS analyses were conducted, clear concentration trends at well ABP-1A are evident in the historical data for the organic compounds tetrachloroethylene, trichloroethylene, and carbon tetrachloride, but not for total chromium, gross alpha, or total lead. Synchronous "spikes" in concentration are observed for the organic compounds, with peaks occurring in late 1993, followed by steady declines in concentration. In all cases, the PWMS samples are highly consistent with the continuation of these trends to the present time. Very few historical measurements exist for total chromium concentration but substantially more have been conducted for gross alpha and total lead, even though many of the results are less than the method detection limit. In all cases, the PWMS analyses are highly consistent with the historical measurements.

Among the naturally occurring analytes, total aluminum, total iron, total calcium, chloride, total manganese, total potassium, and total sodium, no historical concentration trends are observed. The PWMS concentrations are very similar to the historical concentrations for all of these analytes, although few historical analyses have been conducted for total calcium, total iron, total potassium, and total sodium, and certain results are reported as "estimated values" or as "less than the method detection limit." Specific conductance and pH levels obtained from PWMS samples are both highly similar to historical measurements of those parameters and fall well within the historical range.

\subsubsection{ABP-4 Sample Analysis Results (Figure 5)}

As was the case with ABP-1A, the organic compounds tetrachloroethylene, trichloroethylene, and carbon tetrachloride exhibit historical concentration trends; however other potential contaminants, total chromium, gross alpha, and total lead, do not. Synchronous "spikes" in concentration are observed for the organic compounds, with peaks occurring in late 1993, followed by steady declines in concentration. The "spike" is less distinct for carbon tetrachloride due to a number of the concentrations being reported as estimated concentrations. In all cases, though, the PWMS samples are highly consistent with the continuation of the historical concentration trends to the present time. For the other potential contaminants, no concentration trends are seen; however, the PWMS measurements are highly consistent with the historical measurements. 

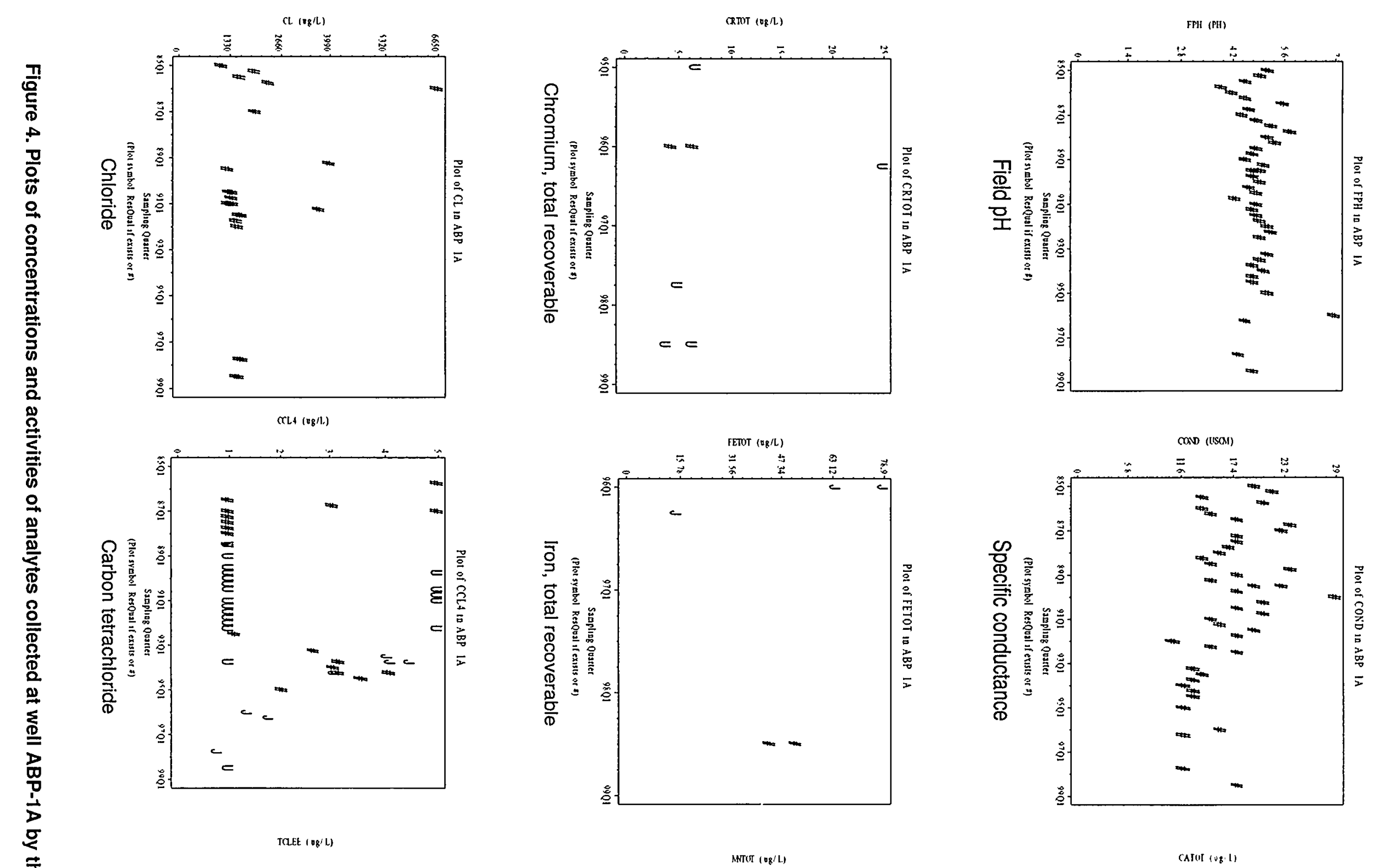

TCEE $\log /$ L)
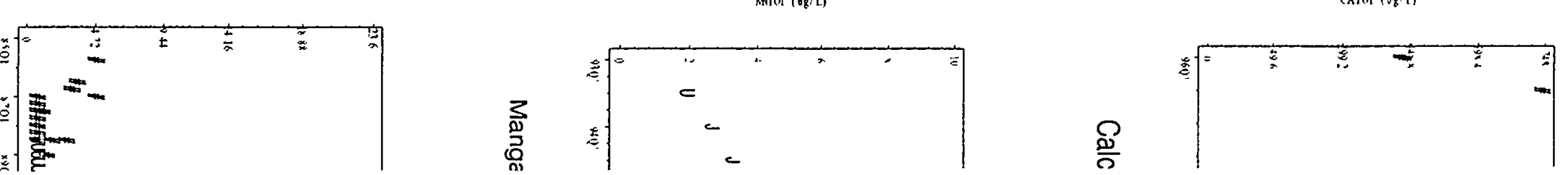
Evaluation of the Validity of Groundwater Samples Obtained Using the PWMS at SRS

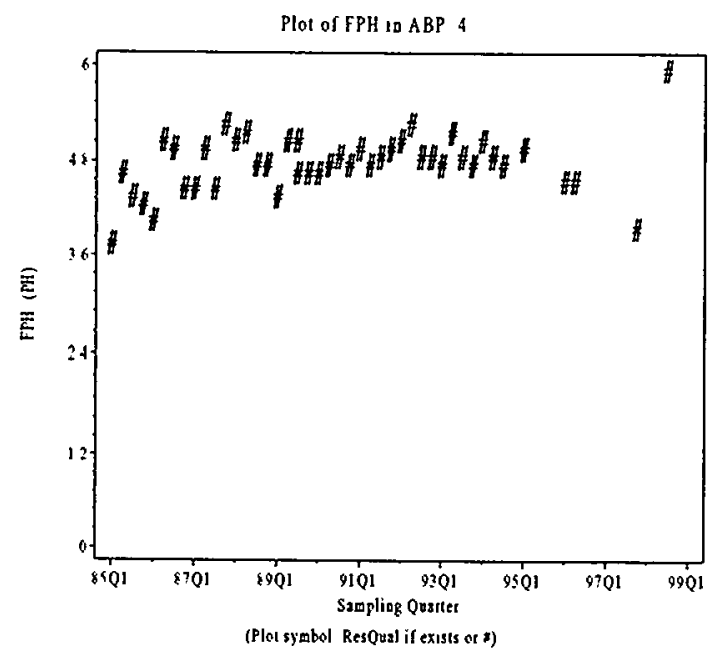

Field $\mathrm{pH}$

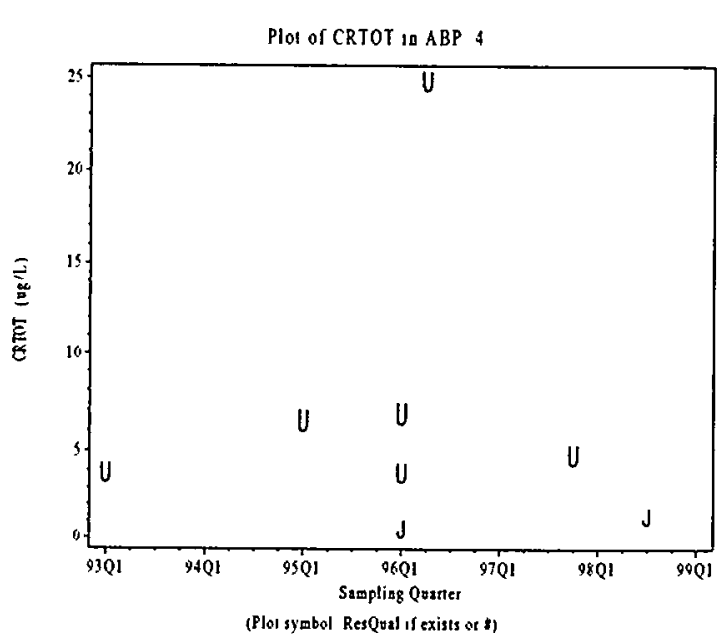

Chromium, total recoverable

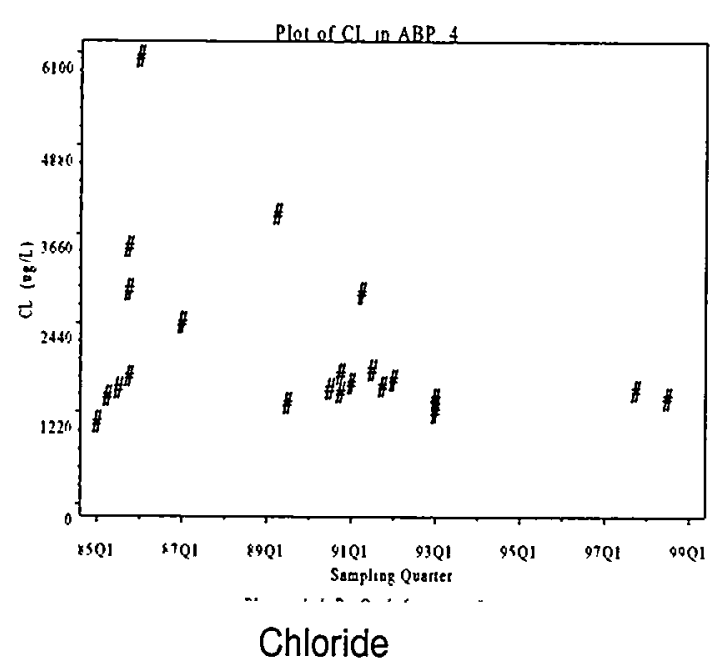

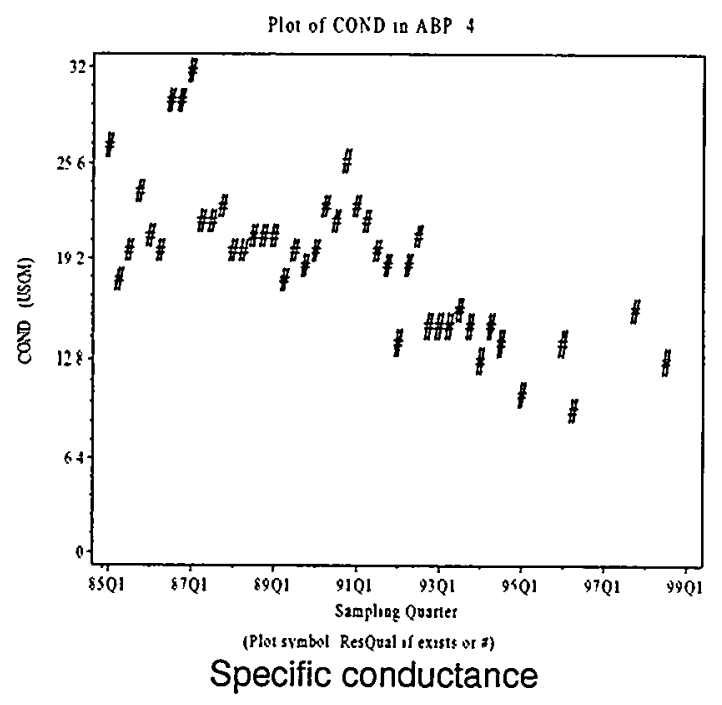
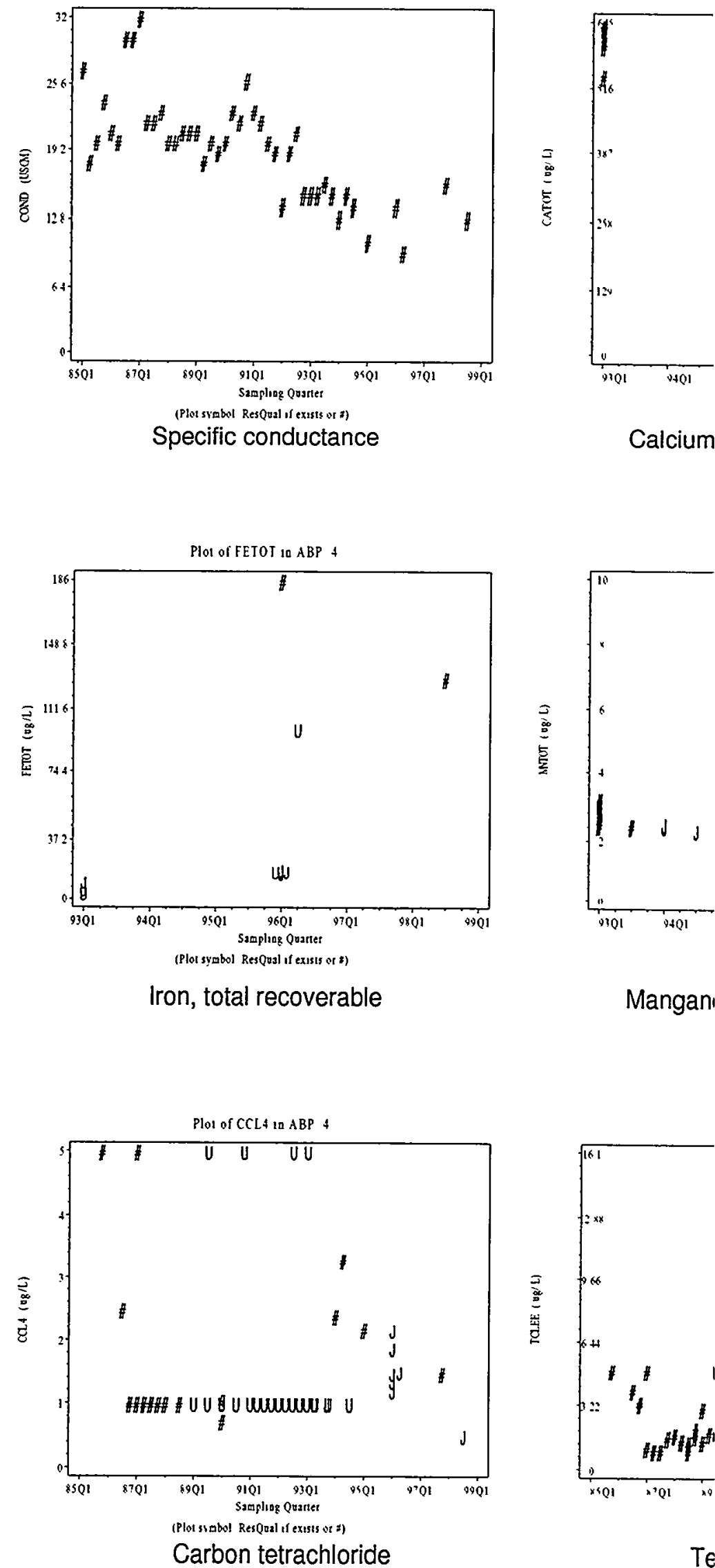

Figure 5. Plots of concentrations and activities of analytes collected at well ABP-4 by the PWMS (in rec 
Among the naturally occurring analytes, total aluminum, total iron, total calcium, chloride, total manganese, total potassium, and total sodium, no historical concentration trends are evident. The PWMS concentrations are all highly similar to the historical concentrations, falling within the range of historical concentrations. In the case of total aluminum, all historical concentrations are reported as "less than the method detection limit;" however, the PWMS results are consistent with these measurements. Specific conductance measurements obtained from PWMS samples are highly similar to historical measurements. With field $\mathrm{pH}$, one of the PWMS sample measurements falls within the historical range while the other is slightly above the highest recorded $\mathrm{pH}$.

\subsubsection{ARP-3 Sample Analysis Results (Figure 6)}

Among the potential contaminants for which PWMS analyses were conducted, no clear concentration trends are occurring at the present time. Elevated concentrations for tetrachloroethylene and trichloroethylene occurred in distinct "spikes" that reached their peak levels in 1990. After 1991, however, concentrations returned to lower levels. The other potential contaminants carbon tetrachloride, total chromium, gross alpha, and total lead do not exhibit historical concentration trends. The PWMS analysis results for tetrachloroethylene and trichloroethylene are highly similar to the lower historical concentrations observed after 1991, while results for the other potential contaminants are also very similar to historical concentrations.

Among the naturally occurring analytes, total aluminum, total iron, total calcium, chloride, total manganese, total potassium, and total sodium, no historical concentration trends are evident. Although only a few historical analyses have been conducted to determine concentrations of total calcium, total iron, total potassium, and total sodium at this well, PWMS analytical results are quite similar to these historical values. Only total sodium and total calcium have concentrations that fell slightly out of the ranges for the few historical concentration values measured. Specific conductance and $\mathrm{pH}$ levels obtained from PWMS samples are both highly similar to historical measurements of those parameters and fall well within the historical range. 


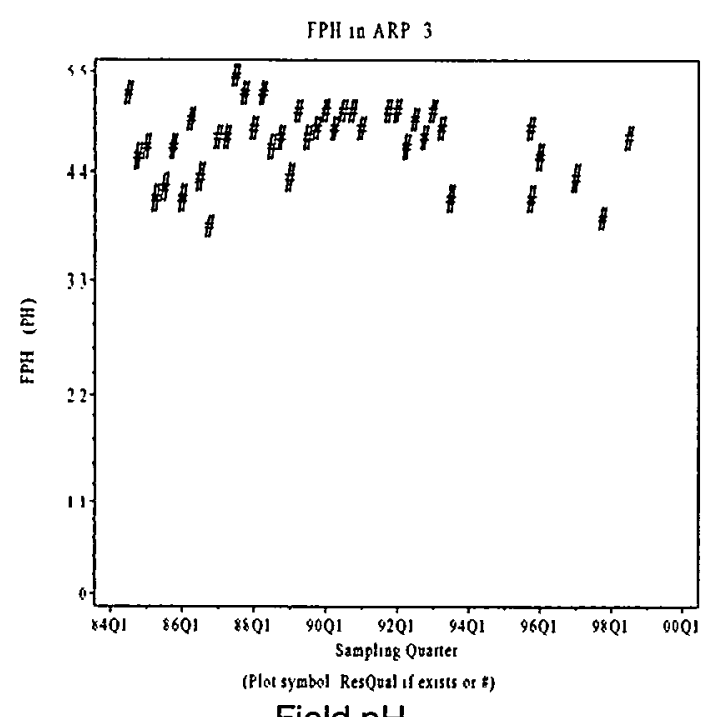

Field pH

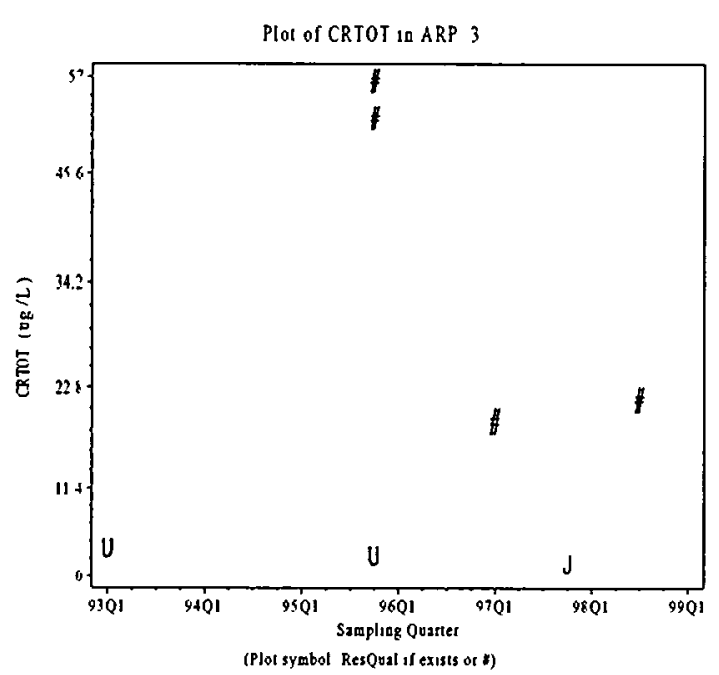

Chromium, total recoverable

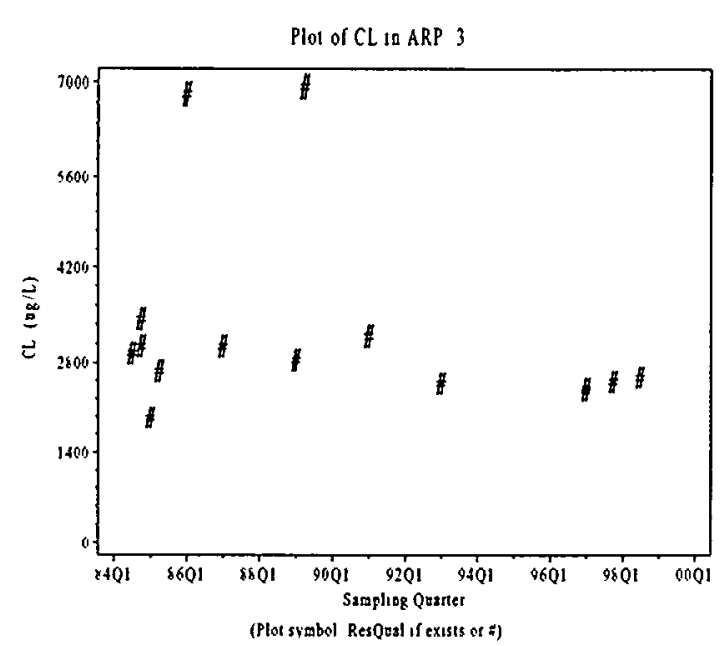

Chloride
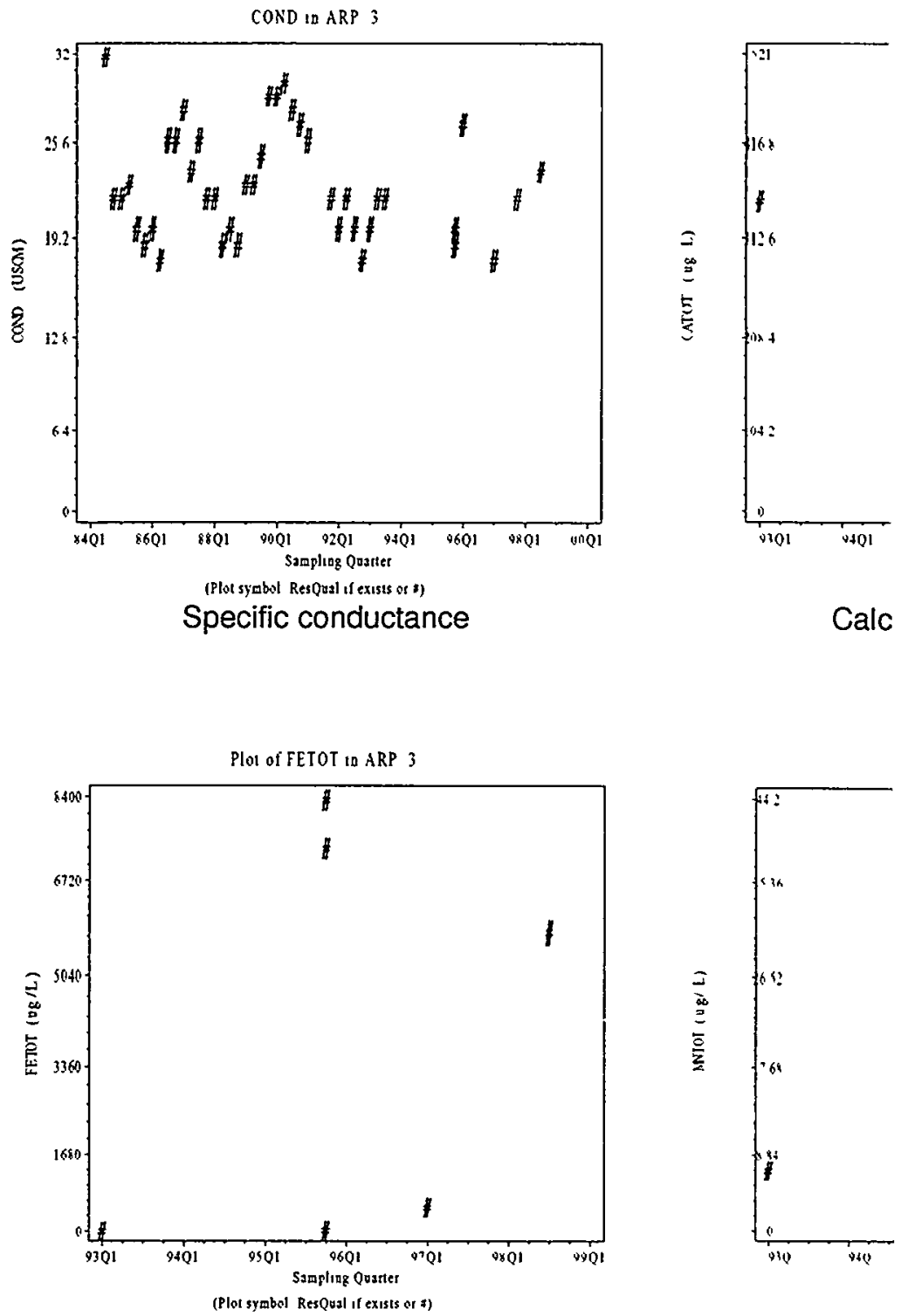

Iron, total recoverable

Mangan
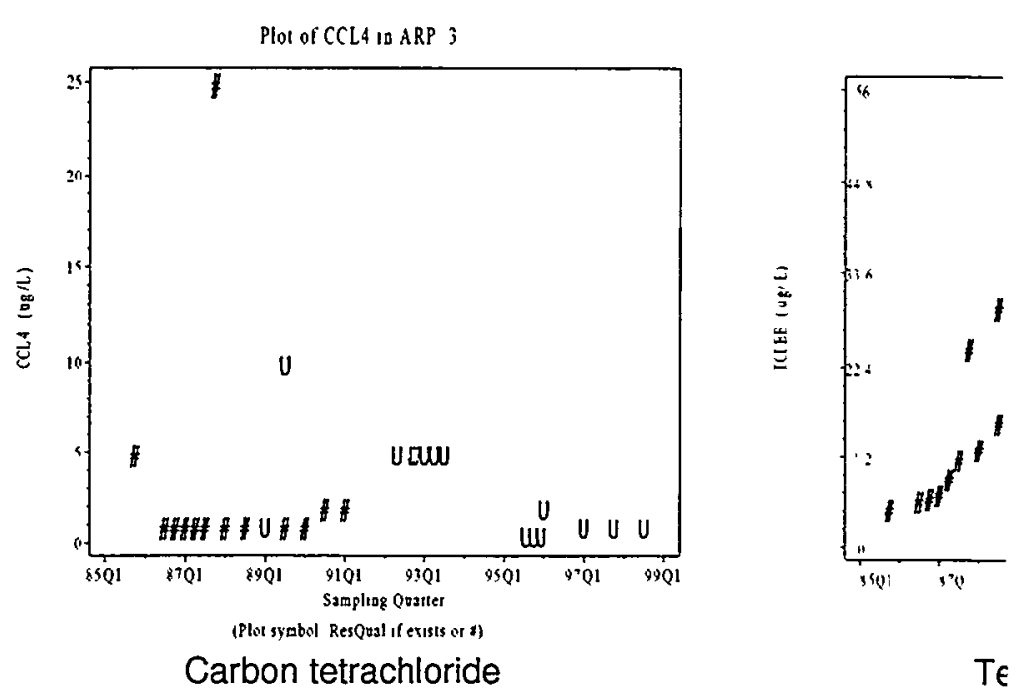

Figure 6. Plots of concentrations and activities of analytes collected at well ARP-3 by the PWMS (in res 


\subsubsection{BGO-33C Sample Analysis Results (Figures 7, 8, and 9)}

Among the potential contaminants for which PWMS analyses were conducted, clear concentration trends are evident for the organic compounds tetrachloroethylene, trichloroethylene (Figure 7), and tritium (Figure 8) but not for total chromium, gross alpha, total lead, or carbon tetrachloride (Figure 7). PWMS sample analyses extended the concentration trends to the present time for tetrachloroethylene, trichloroethylene, and tritium as can be seen on the regression plots of Figure 9. The PWMS concentrations fall almost exactly on the projected line for tetrachloroethylene and tritium but fall slightly above this line for trichloroethylene. For the other potential contaminants, carbon tetrachloride, total chromium, total lead, and gross alpha, the PWMS concentrations are very consistent with historical concentrations (Figure 7).

Among the naturally occurring analytes, total aluminum, total iron, total calcium, chloride, total manganese, total potassium, and total sodium (Figures 7 and 9), historical concentrations suggest that trends exist for total calcium, total sodium, chloride, and total manganese (see Figure 9). Once again, PWMS-derived concentrations appear to fall upon the line that projects these trends to the present and are thus regarded as highly consistent with the trends. PWMS analyses provided total iron concentrations that are estimated or "less-than-the-method-detection-limit," but appear to be consistent with the historical concentrations. PWMS concentrations for total potassium are quite similar to the historical concentrations, although a single analysis had a concentration that was slightly lower. Specific conductance and $\mathrm{pH}$ levels obtained from PWMS samples are both highly similar to historical measurements of those parameters and fall well within the historical range.

\subsection{Statistical Evaluation}

A statistical evaluation was performed to provide validation for the graphical comparisons described in Section 3.1. A report detailing the statistical evaluation including data used for the comparison and numerical results is provided in Appendix B. The PWMS data evaluated included analytical results from samples collected on the influent side of the PWMS after stabilization of field parameters (representative of aquifer conditions) and also "effluent" samples collected when well purge water from the PWMS tank was draining back to the aquifer. As the PWMS tank contained all water purged from the well, analytical results are representative of both the groundwater in the aquifer being sampled as well as stagnant groundwater remaining in the well between sampling events. 


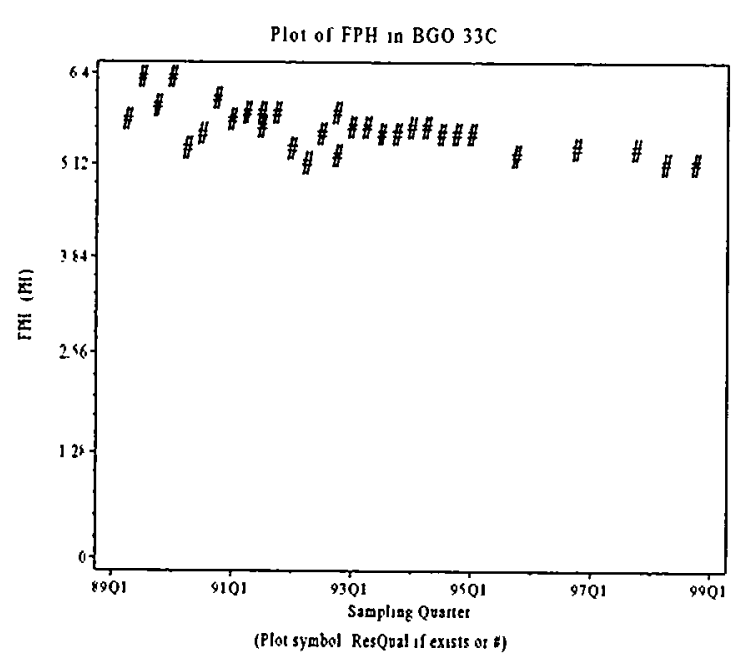

Field $\mathrm{pH}$

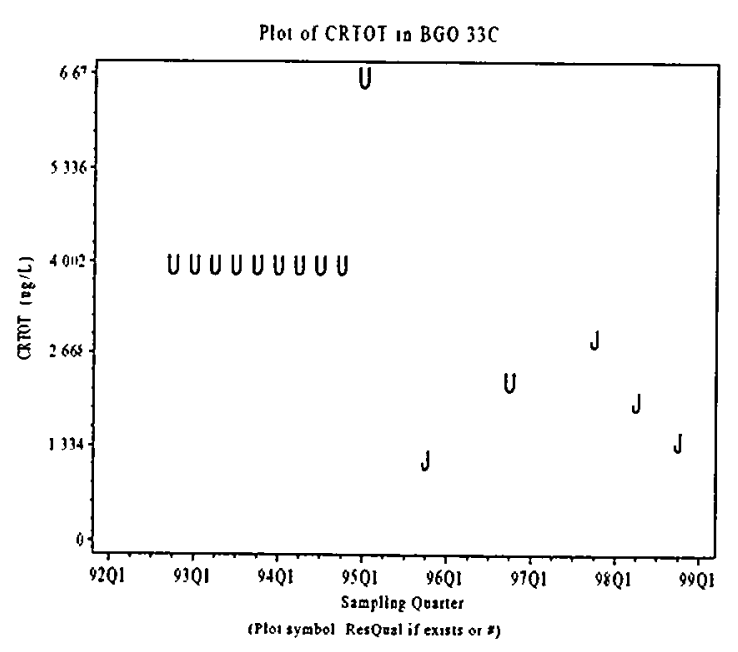

Chromium, total recoverable

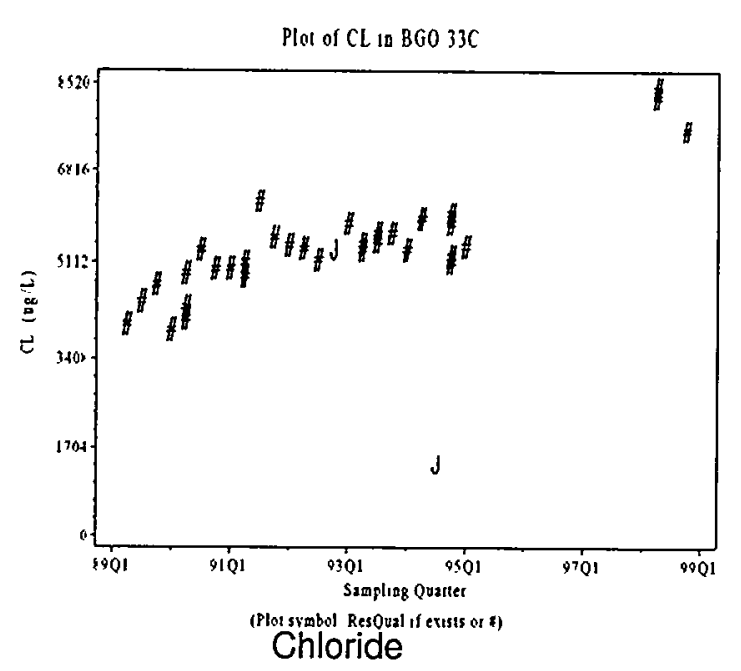

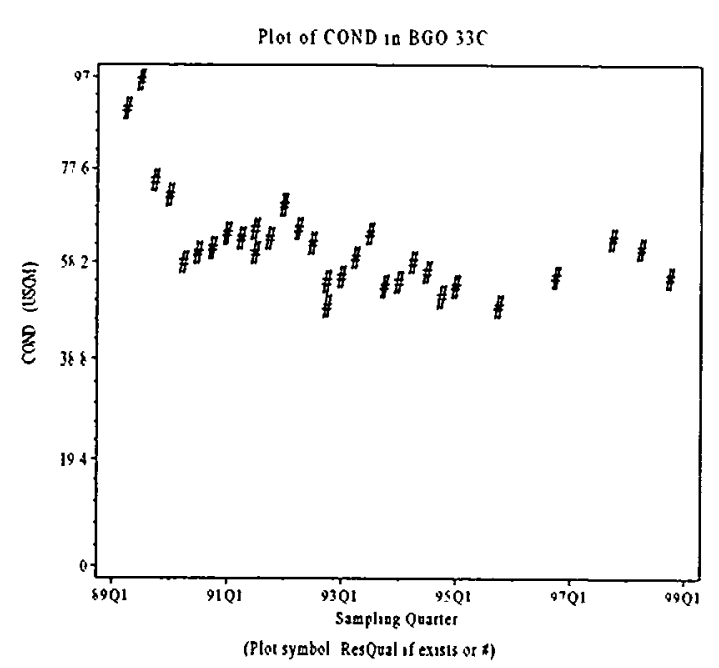

Specific conductance

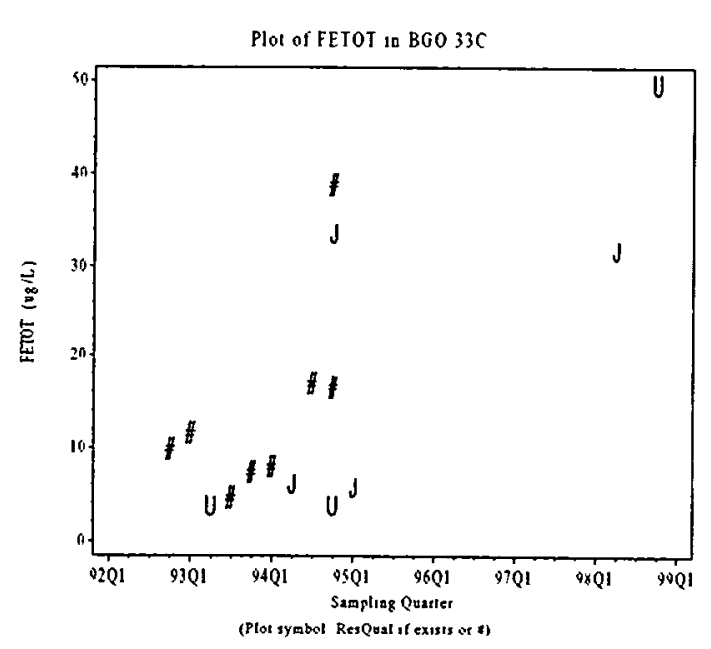

Iron, total recoverable

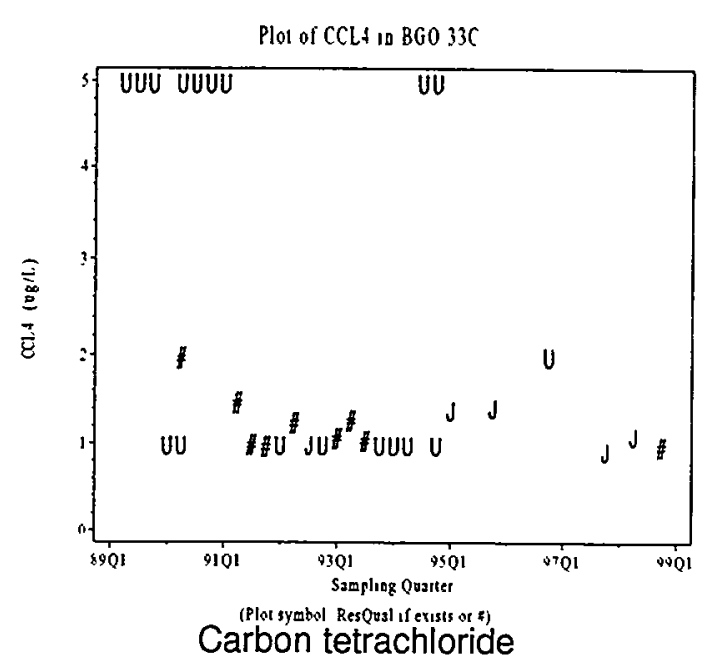

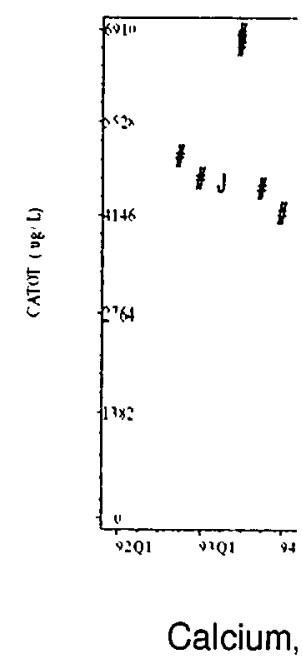

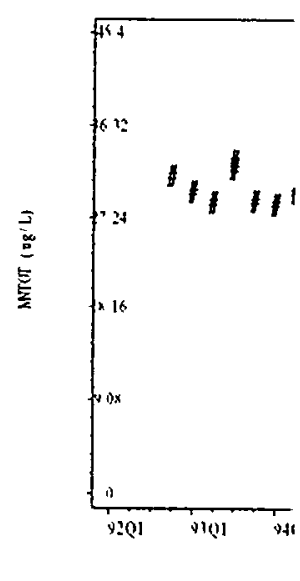

Mangane

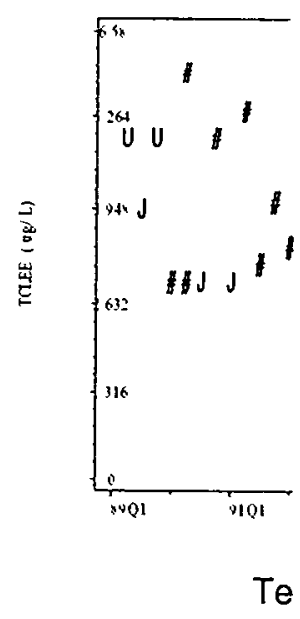

Figure 7. Plots of concentrations and activities of analytes collected at well BGO-33C by the PWMS (in 


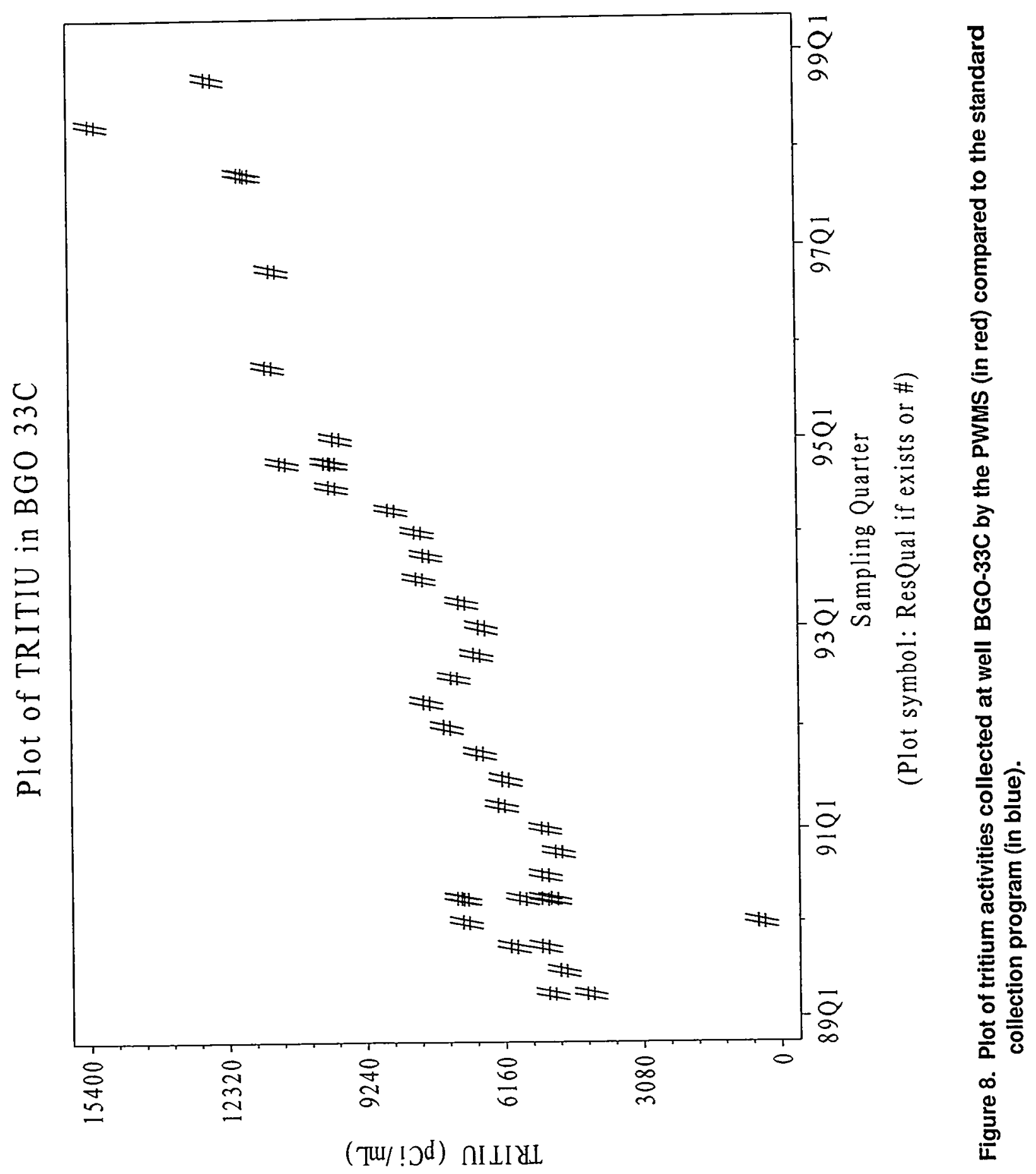



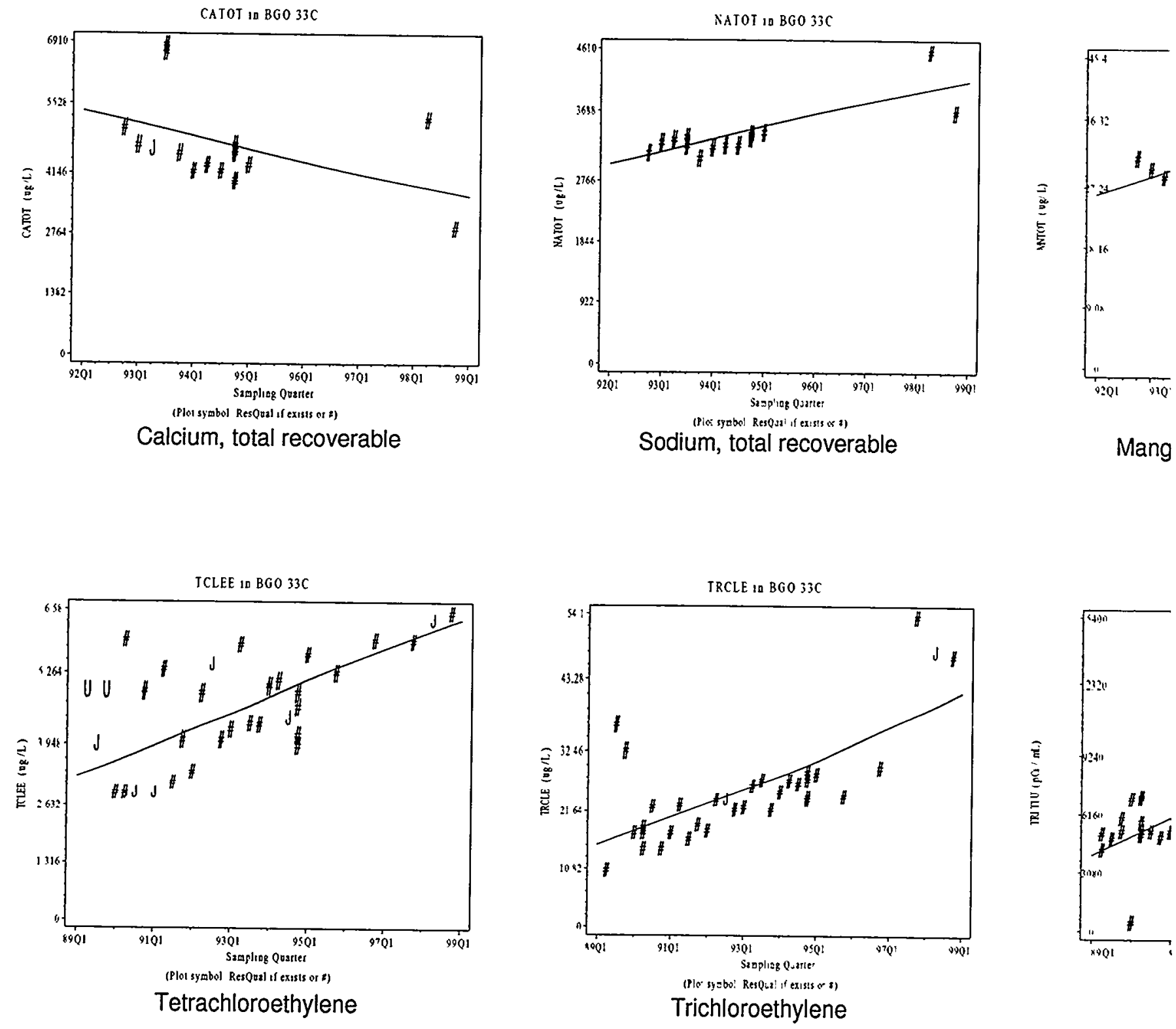

Figure 9. Regression plots of concentrations and activities of analytes collected at well BGO-33C by thi 
The statistical evaluation found that, over all the 15 analytes compared, there is no difference between analytical results from samples collected with the PWMS vs. collections made with the standard sampling program for wells ABP-1A, ABP-4, and ARP-3. There was no statistical difference found between analytical results collected with the two sampling methods for well BGO-33C except for one analyte, total recoverable manganese. This result for manganese for BGO-33C is most likely explained by the fact that the analytical results of PWMS sampling appear to extend an historical trend (see Figures 7 and 9) but there is a sampling gap of three to four years (1995-1998) between the PWMS and historic analytical results for this analyte.

\subsection{Tracer Study}

The Potassium bromide tracer was introduced into well BGO-33C in June 1998 (Figures 10 and 11 and Appendix A). Initial concentrations were $3,300 \mu \mathrm{g} / \mathrm{L}$ for bromide and $2,090 \mu \mathrm{g} / \mathrm{L}$ for potassium. The resulting tracer ion bromide, which is mobile and conservative in the groundwater flow system, was not detected above the method detection limit at the subsequent sampling event that was conducted 180 days later in December 1998 (Figure 10 and Appendix A). It is not known whether the bromide concentration returned to background levels because the sample quantitation limit for that analysis was greater than the (estimated, J qualifier) background level of December 1997. On the other hand, the tracer ion potassium, which was injected at a concentration of $2,090 \mu \mathrm{g} / \mathrm{L}$ was detected at a concentration of $768 \mu \mathrm{g} / \mathrm{L}$ in the sample collected 180 days later, a level highly consistent with background potassium concentrations for that well (Figure 11 and Appendix A). These results support the conclusion that natural groundwater flow carried the tracer solution sufficiently far away from the screen zone at BGO-33C that it was not drawn back into the well during the next sampling event that occurred 180 days later. 


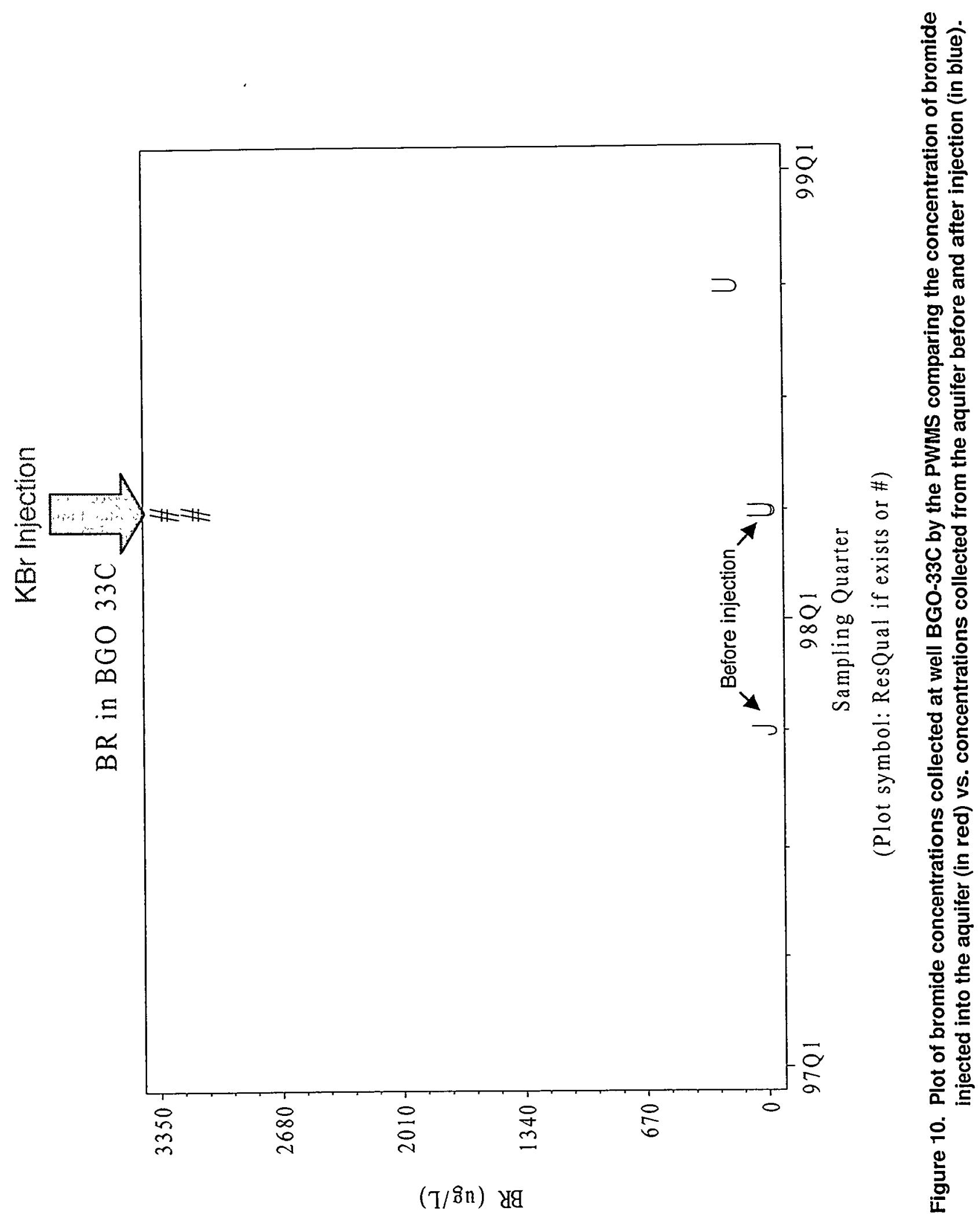




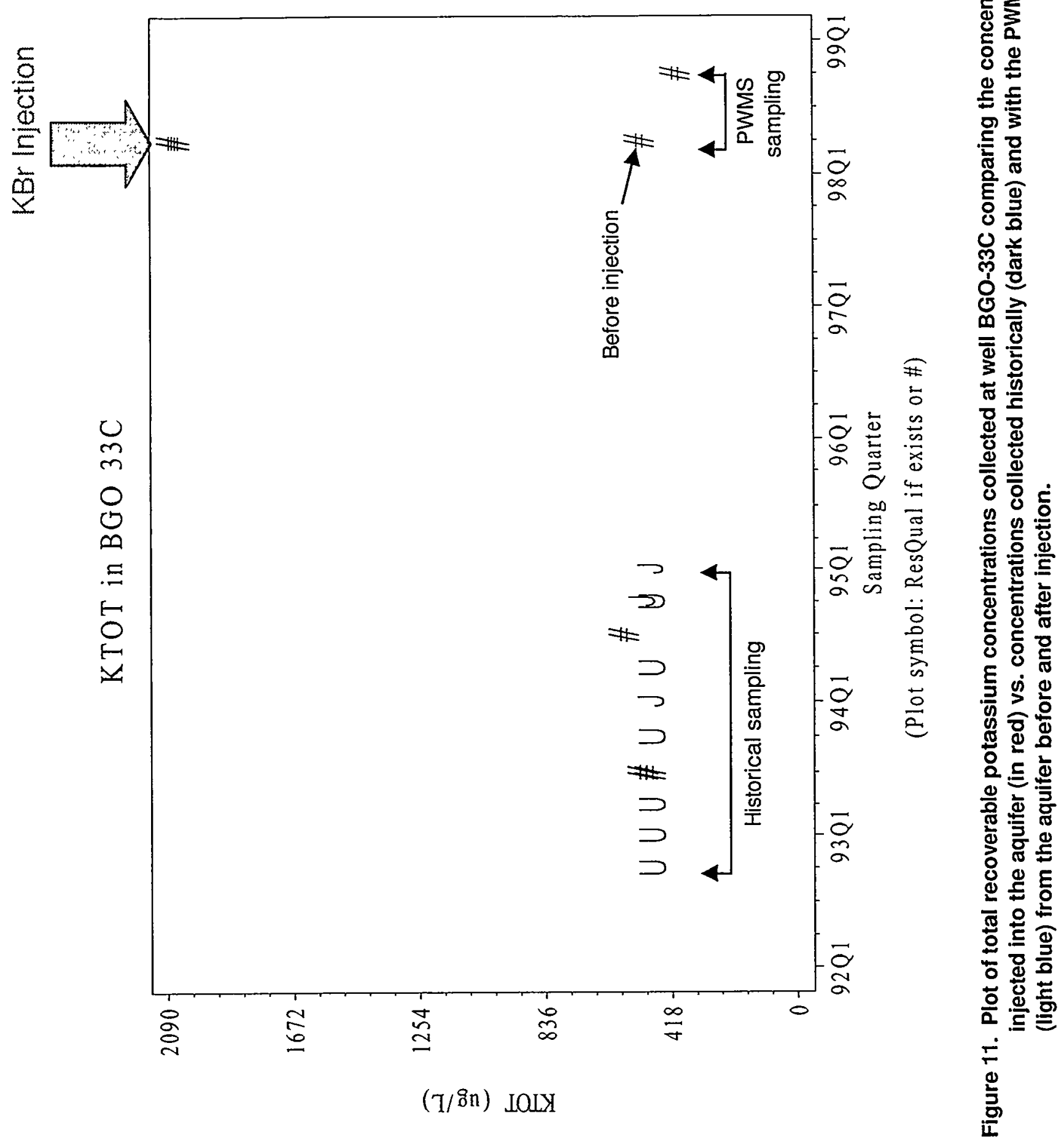




\subsection{CONCLUSIONS}

The analysis of the groundwater chemical concentrations indicates that the PWMS sampling methodology acquired representative groundwater samples at monitoring wells $A B P-1 A, A B P-4, A R P-3$ and $B G O-$ 33C. Representative groundwater samples are achieved if the PWMS does not resample groundwater that has been purged and returned during a previous sampling event. Initial screening calculations, conducted prior to the selection of these four wells, indicated that groundwater velocities were high enough under natural hydraulic gradients to preclude resampling from occurring at the time intervals that were used at each well. Corroborating evidence included the high degree of similarity between analyte concentrations derived from the PWMS samples and from the historical analyte concentrations as well as the fact that PWMS data extend all previously existing concentration trends to the present time. The latter line of evidence is the most powerful in demonstrating that representative samples are being acquired by the PWMS because it is highly unlikely that previously existing concentration trends would continue if resampling had occurred.

The fact that potassium concentrations returned to background levels in the PWMS sampling event that occurred 180 days after the introduction of the concentrated potassium bromide tracer in well BGO-33C indicates that groundwater was not resampled during that event. The sharp drop-off in bromide concentrations is also consistent with this contention.

The PWMS can collect valid and representative groundwater samples if a sufficient amount of time is allowed for natural groundwater movement to sweep water that is returned to the aquifer sufficiently far away from the well such that it is not drawn into the well during subsequent sampling events. For wells completed in aquifers having unusually low groundwater velocities the minimum amount of time allowed to pass between samples may have to be longer to ensure this does not occur. In most wells at SRS a 90day period between wells appears to be sufficient. In other wells a period of 180 days may be the minimum time period between subsequent sampling events. Most wells routinely sampled in the SRS groundwater monitoring are sampled on an annual or semi-annual basis (Table 4); therefore, the use of PWMS is likely to be appropriate at those wells. Other wells are sampled on a quarterly basis and will require an evaluation on an individual basis to determine the suitability of PWMS for deployment. 
Evaluation of the Validity of Groundwater Samples Obtained

WSRC-TR-99-00042

Using the Purge Water Management System (PWMS) at SRS

Revision 0

March 1999

Table 4. Frequency of sampling of SRS monitoring wells.

\begin{tabular}{|c|c|}
\hline Number of Wells & Sampling Frequency \\
\hline 366 & $1 \mathrm{x} /$ year \\
\hline 435 & $2 \mathrm{x} /$ year \\
\hline 49 & $3 \mathrm{x} /$ year \\
\hline 312 & $4 \mathrm{x} /$ year \\
\hline
\end{tabular}




\subsection{REFERENCES}

Hiergesell, R.A., J.E. Cardoso-Neto, and D.W. Williams, 1996. Evaluation of the Purge Water Management System (PWMS) Monitor Well Sampling Technology at SRS, WSRC-TR-96-0416, Westinghouse Savannah River Company, Aiken, South Carolina.

WSRC, 1994. Investigation Derived Waste Management Plan, WSRC-RP-94-1227, Revision 2, Westinghouse Savannah River Company, Aiken, South Carolina.

WSRC, 1992. Hydrogeologic Data Collection, Chapter 15, Sampling Groundwater Monitoring Wells, Procedure Manual WSRC-3Q5, Westinghouse Savannah River Company, Aiken, South Carolina. 


\section{DATA CODES USED IN THE WELL SAMPLE ANALYSIS RESULTS}

\begin{tabular}{|c|c|}
\hline \multicolumn{2}{|c|}{ Analyte Codes } \\
\hline Code & Analyte \\
\hline FPH & Field pH \\
\hline COND & Specific conductance \\
\hline CATOT & Calcium, total recoverable \\
\hline KTOT & Potassium, total recoverable \\
\hline NATOT & Sodium, total recoverable \\
\hline CRTOT & Chromium, total recoverable \\
\hline FETOT & Iron, total recoverable \\
\hline MNTOT & Manganese, total recoverable \\
\hline ALTOT & Aluminum, total recoverable \\
\hline PBTOT & Lead, total recoverable \\
\hline CL & Chloride \\
\hline CCLA & Carbon tetrachloride \\
\hline TCLEE & Tetrachloroethylene \\
\hline TRCLE & Trichloroethylene \\
\hline ALPHAG & Gross alpha \\
\hline TRITIU & Tritium \\
\hline BR & Bromide \\
\hline \multicolumn{2}{|c|}{ Analytical Result Qualifier Codes } \\
\hline Code & Qualification \\
\hline \# & Result has no qualifier - interpret as reported \\
\hline J & Result is estimated quantity \\
\hline U, UI & Result is less than sample quantitation limit \\
\hline
\end{tabular}


WELL ABP-1A DATA 
FPH Results for ABP 1A

Data Retrieved by AWSA v1 from GIMS on 04MAR99 at 10:55

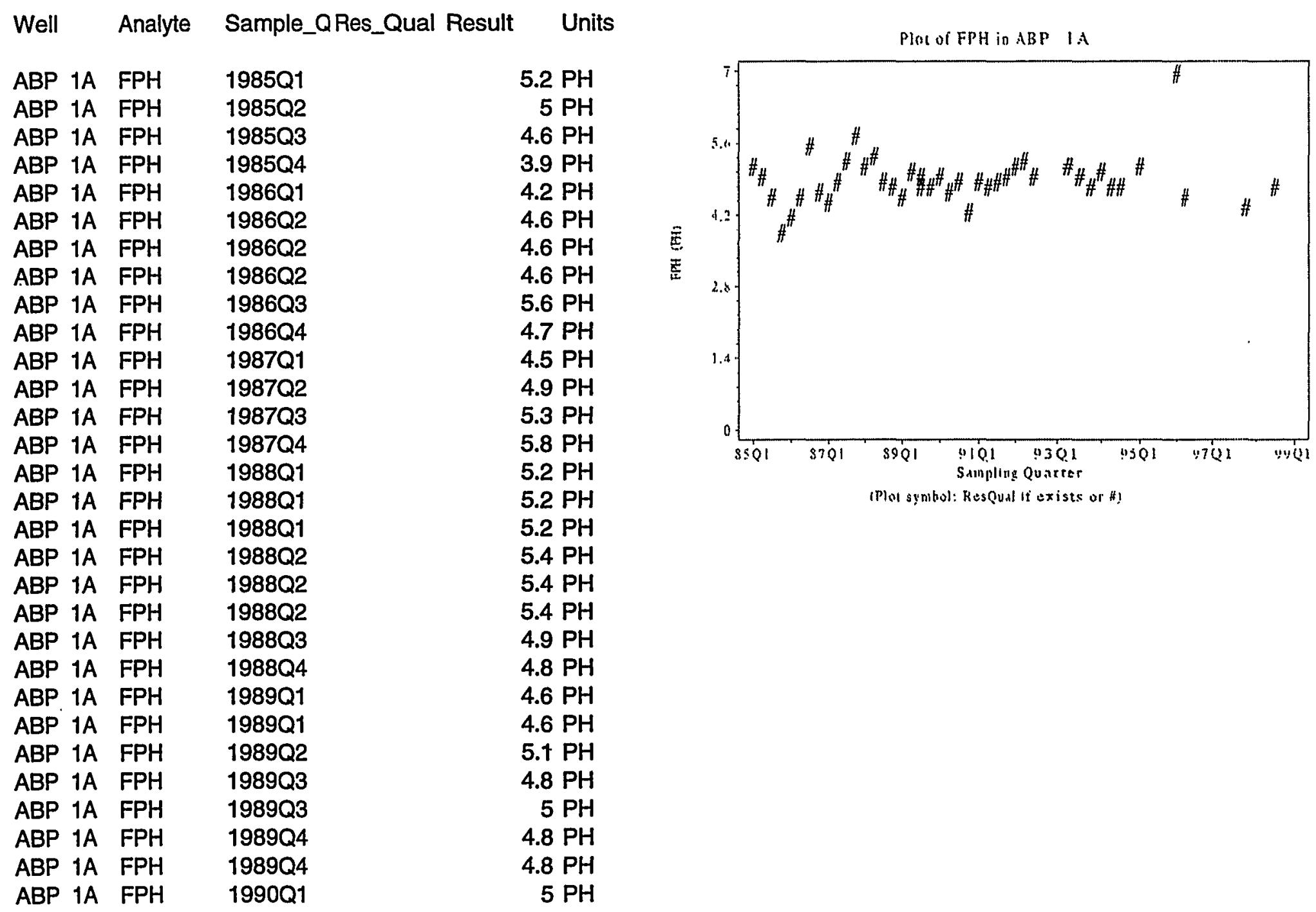




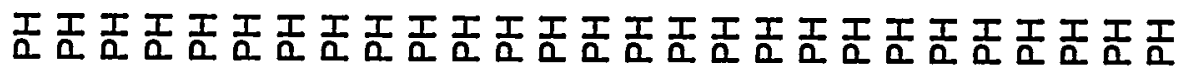

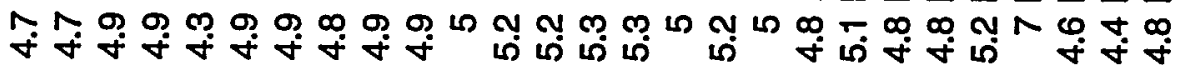

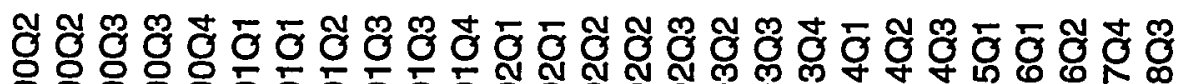
\%

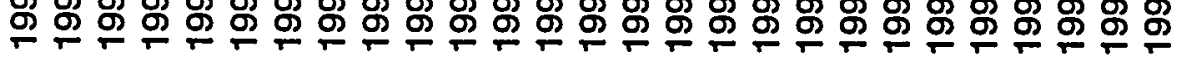

IIIIIIIIIIIIIIIIIIIIIIIIIIIIIIII

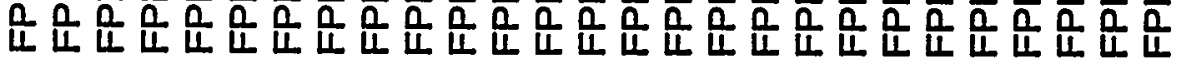
$\llbracket \leq \leq \leq \leq \leq \leq \leq \leq \leq \leq \leq \leq \leq \leq \leq \leq \leq \leq \leq \leq \leq \leq \leq \leq \leq \leq$

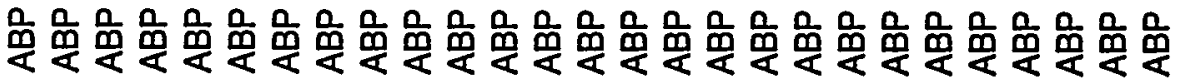




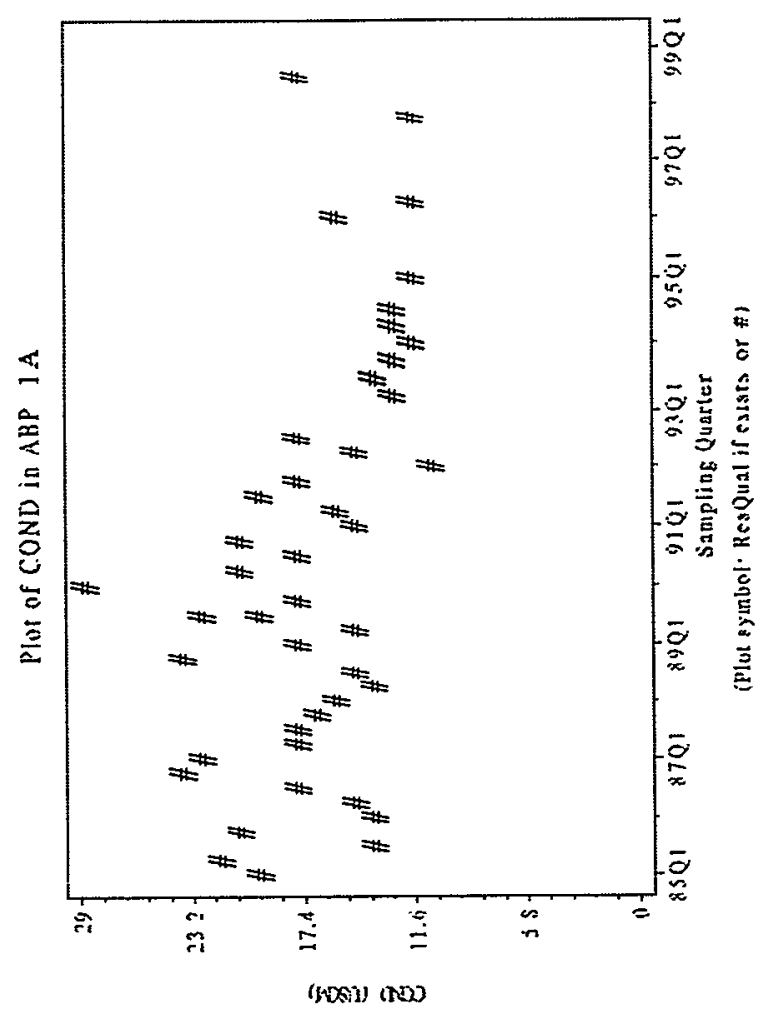

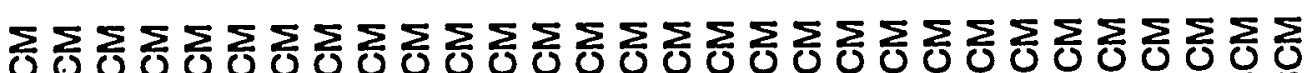

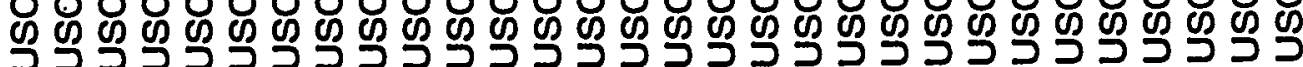

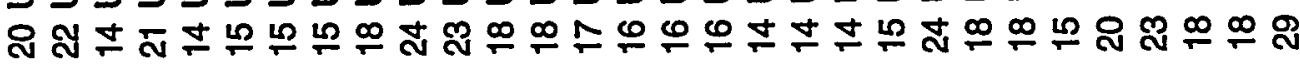

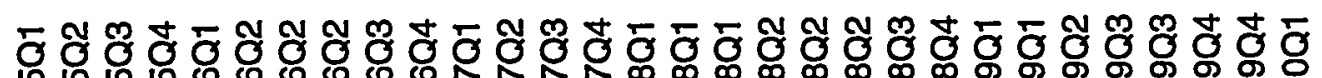
识识

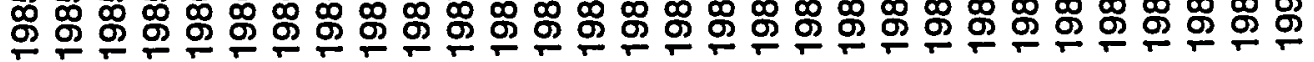

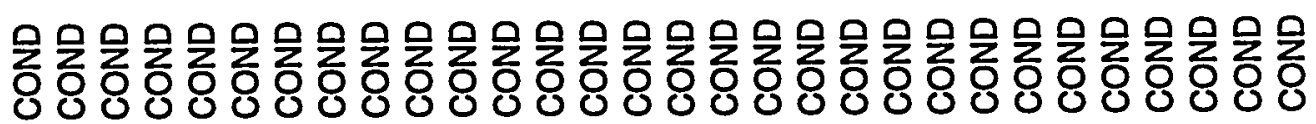
$\leq \leq \leq \leq \leq \leq \leq \leq \leq \leq \leq \leq \leq \leq \leq \leq \leq \leq \leq \leq \leq \leq \leq \leq \leq \leq \leq \leq \leq \leq$

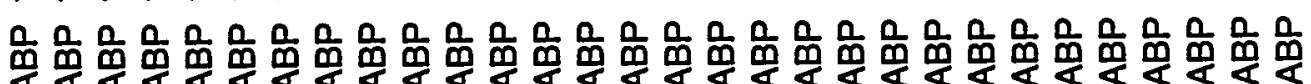




\begin{tabular}{|c|c|c|c|}
\hline$A B P$ 1A & COND & 1990Q2 & 21 USCM \\
\hline$A B P 1 A$ & COND & 1990Q2 & 21 USCM \\
\hline ABP $1 \mathrm{~A}$ & COND & $1990 Q 3$ & 18 USCM \\
\hline$A B P 1 A$ & COND & 1990Q3 & 18 USCM \\
\hline$A B P 1 A$ & COND & 1990Q4 & 21 USCM \\
\hline$A B P \quad 1 A$ & COND & 1991Q1 & 15 USCM \\
\hline ABP $1 A$ & COND & 1991Q1 & 15 USCM \\
\hline$A B P 1 A$ & COND & 1991Q2 & 16 USCM \\
\hline ABP $1 A$ & COND & 1991Q3 & 20 USCM \\
\hline$A B P 1 A$ & COND & 1991Q3 & 20 USCM \\
\hline$A B P 1 A$ & COND & 1991Q4 & 18 USCM \\
\hline$A B P 1 A$ & COND & 1992Q1 & 11 USCM \\
\hline$A B P \quad 1 A$ & COND & 1992Q1 & 11 USCM \\
\hline$A B P 1 A$ & COND & 1992Q2 & 15 USCM \\
\hline ABP $1 \mathrm{~A}$ & COND & 1992Q2 & 15 USCM \\
\hline$A B P \quad 1 A$ & COND & 1992Q3 & 18 USCM \\
\hline$A B P 1 A$ & COND & 1993Q2 & 13 USCM \\
\hline$A B P \quad 1 A$ & COND & 1993Q3 & 14 USCM \\
\hline$A B P 1 A$ & COND & 1993Q4 & 13 USCM \\
\hline$A B P 1 A$ & COND & 1994Q1 & 12 USCM \\
\hline$A B P 1 A$ & COND & 1994Q2 & 13 USCM \\
\hline ABP $1 \mathrm{~A}$ & COND & 1994Q3 & 13 USCM \\
\hline$A B P 1 A$ & COND & 1995Q1 & 12 USCM \\
\hline$A B P \quad 1 A$ & COND & 1996Q1 & 16 USCM \\
\hline$A B P 1 A$ & COND & 1996Q2 & 12 USCM \\
\hline$A B P \quad 1 A$ & COND & 1997Q4 & 12 USCM \\
\hline ABP & COND & 1998Q3 & 18 USCM \\
\hline
\end{tabular}



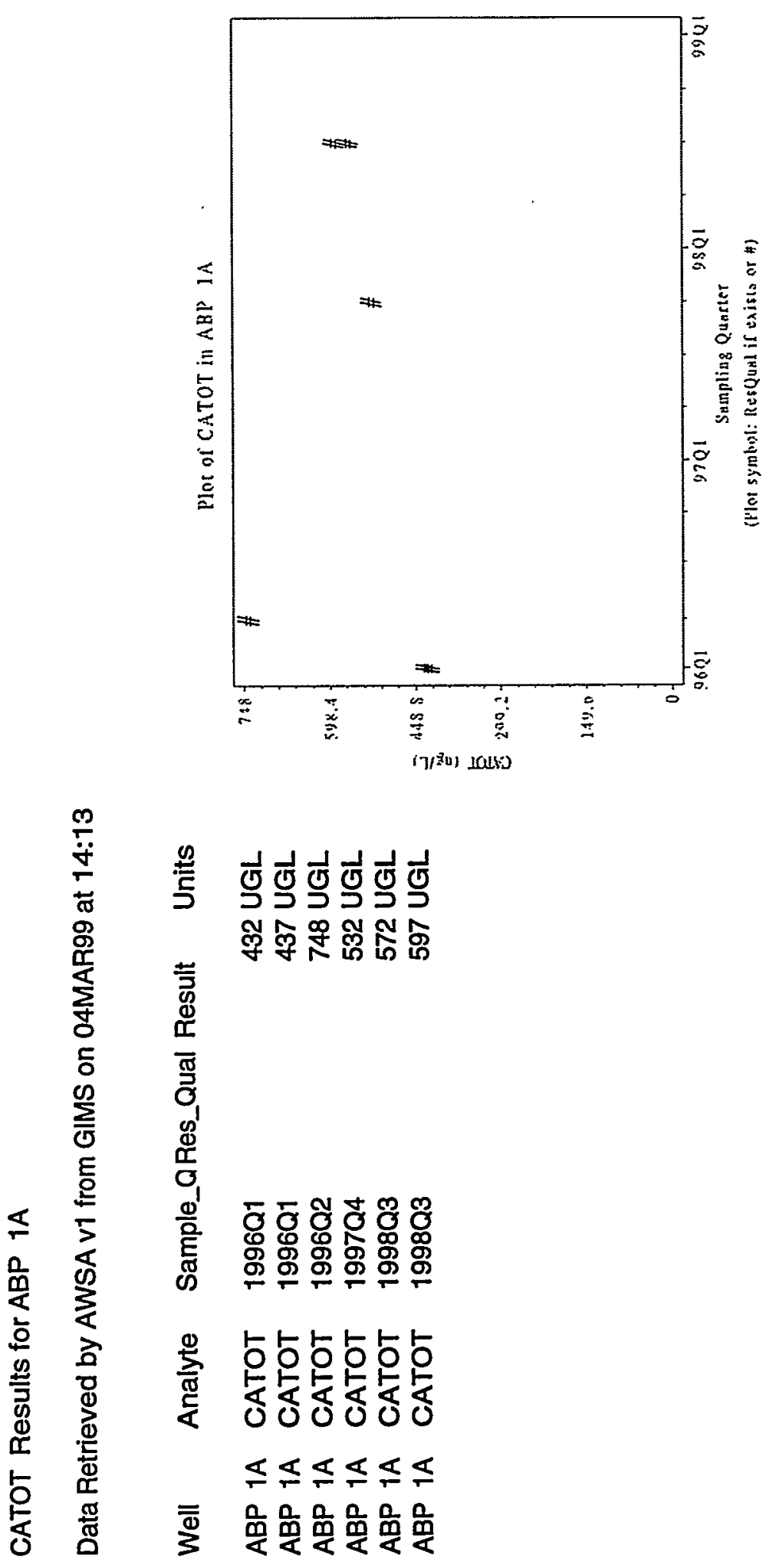
KTOT Results for ABP 1A

Data Retrieved by AWSA v1 from GIMS on 04MAR99 at 14:13

Well Analyte Sample_QRes_Qual Result Units

ABP 1A KTOT 1996Q1 J

ABP 1A KTOT 1996Q1 J

ABP 1A KTOT $1996 Q 2$ U

ABP 1A KTOT 1997Q4

ABP 1A KTOT 1998Q3

ABP 1A KTOT 1998Q3
217 UGL

217 UGL 2000 UGL 271 UGL 238 UGL 252 UGL

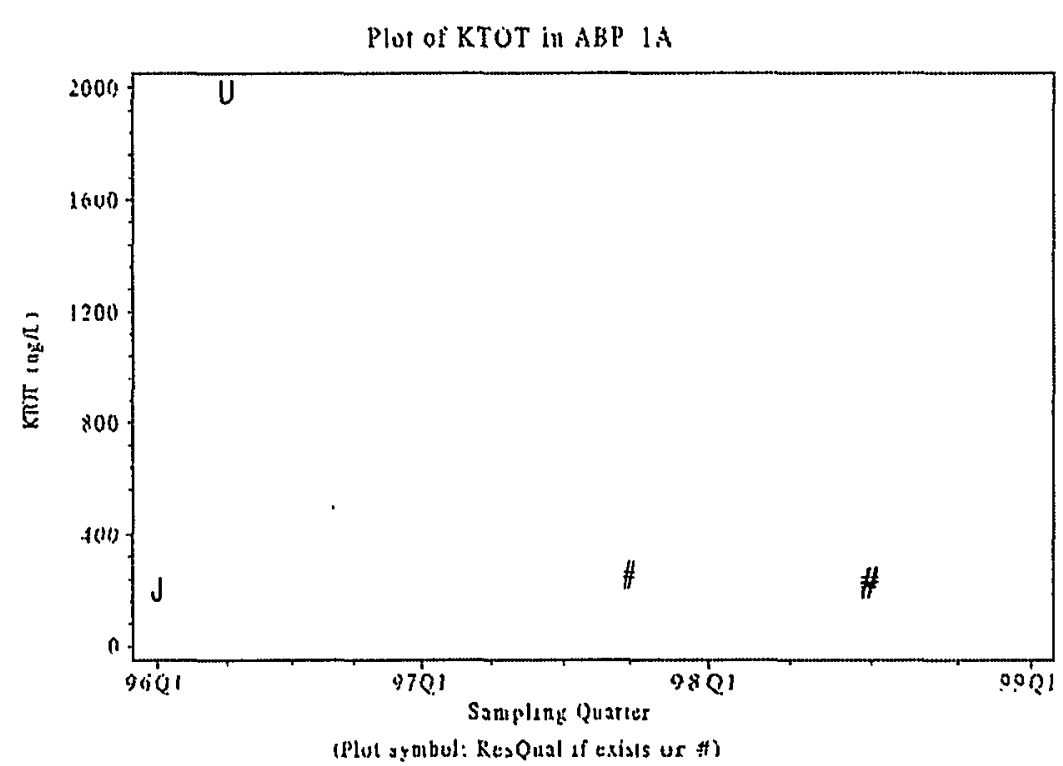


NATOT Results for ABP $1 \mathrm{~A}$

Data Retrieved by AWSA v1 from GIMS on 04MAR99 at 14:13

Well Analyte Sample_QRes_Qual Result Units

ABP 1A NATOT 1996Q1 958 UGL

ABP 1A NATOT 1996Q1 969 UGL

ABP IA NATOT 1996Q2 $1350 \mathrm{UGL}$

ABP 1A NATOT 1997Q4 1020 UGL

ABP 1A NATOT 1998Q3 856 UGL

ABP 1A NATOT 1998Q3 900 UGL 

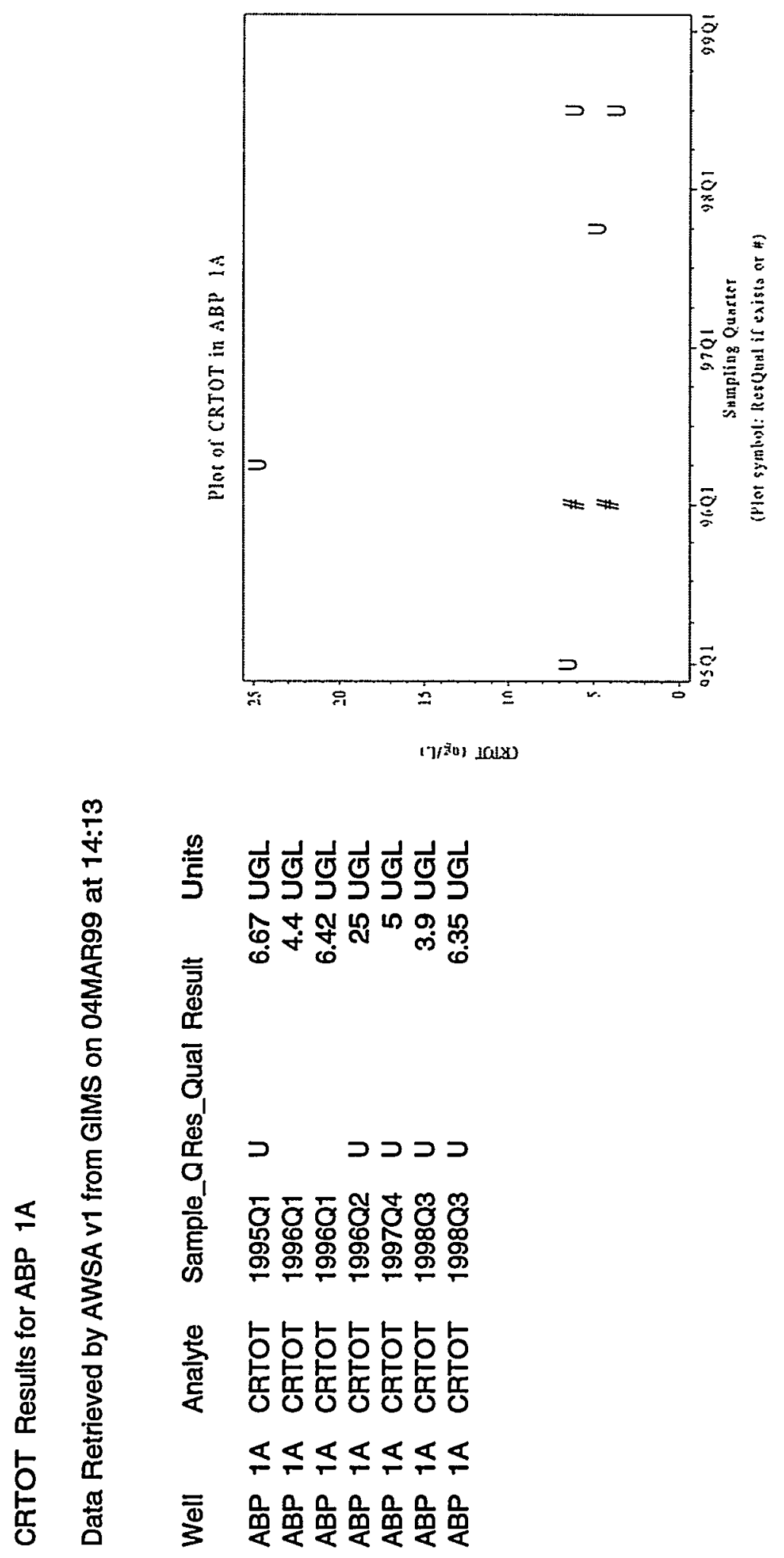
FETOT Results for ABP $1 \mathrm{~A}$

Data Retrieved by AWSA v1 from GIMS on 04MAR99 at 14:13

Well Analyte Sample_QRes_Qual Result Units

ABP 1A FETOT 1996Q1 J $\quad 64.2$ UGL

ABP 1A FETOT 1996Q1 J

ABP 1A FETOT 1996Q2 J 14.9 UGL

ABP 1A FETOT 1998Q3 $44 \mathrm{UGL}$

ABP 1A FETOT 1998Q3 52.2 UGL

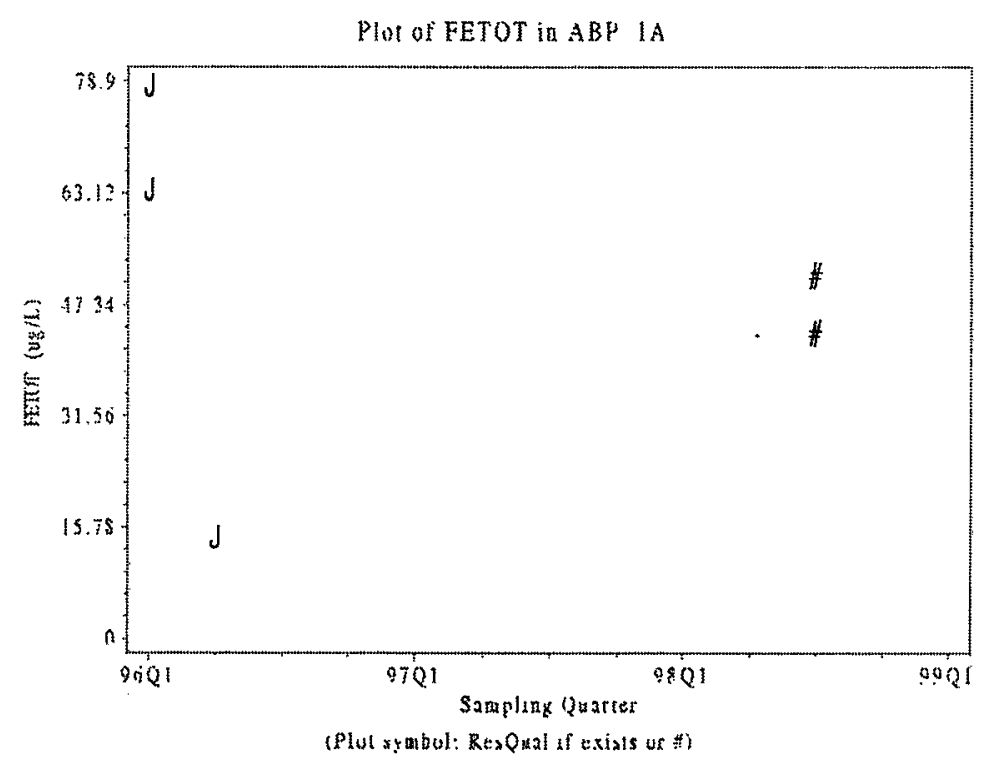


MNTOT Results for ABP 1A

Data Retrieved by AWSA v1 from GIMS on 04MAR99 at 14:13

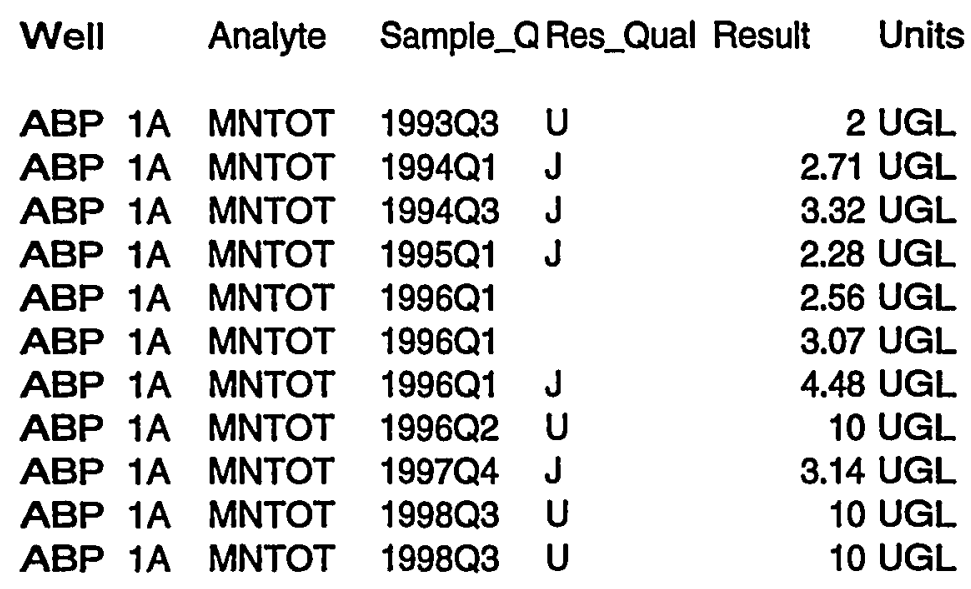

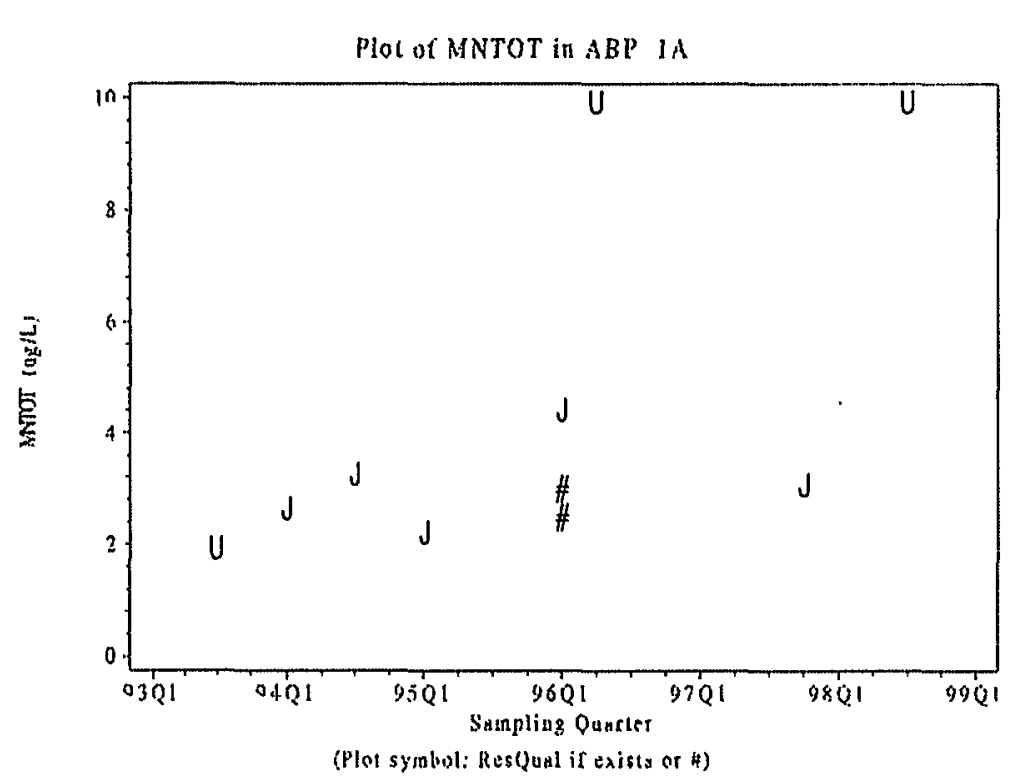


ALTOT Results for ABP $1 \mathrm{~A}$

Data Retrieved by AWSA v1 from GIMS on 04MAR99 at 14:13

Well Analyte Sample_QRes_Qual Result Units

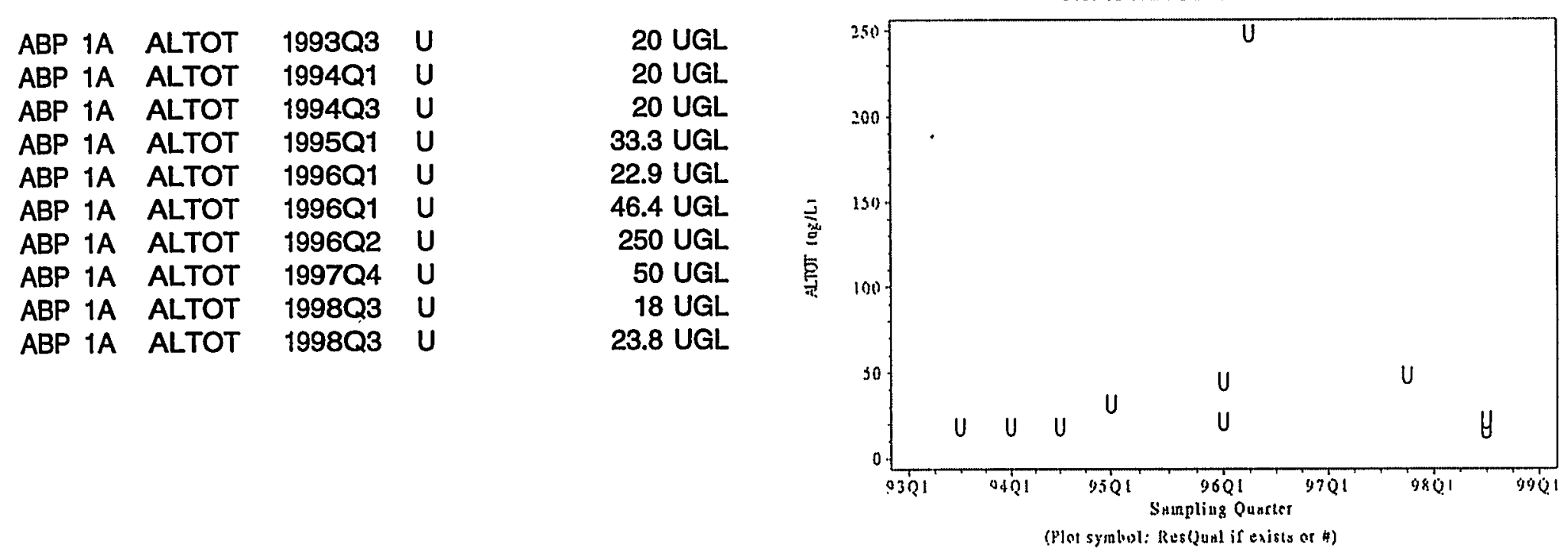



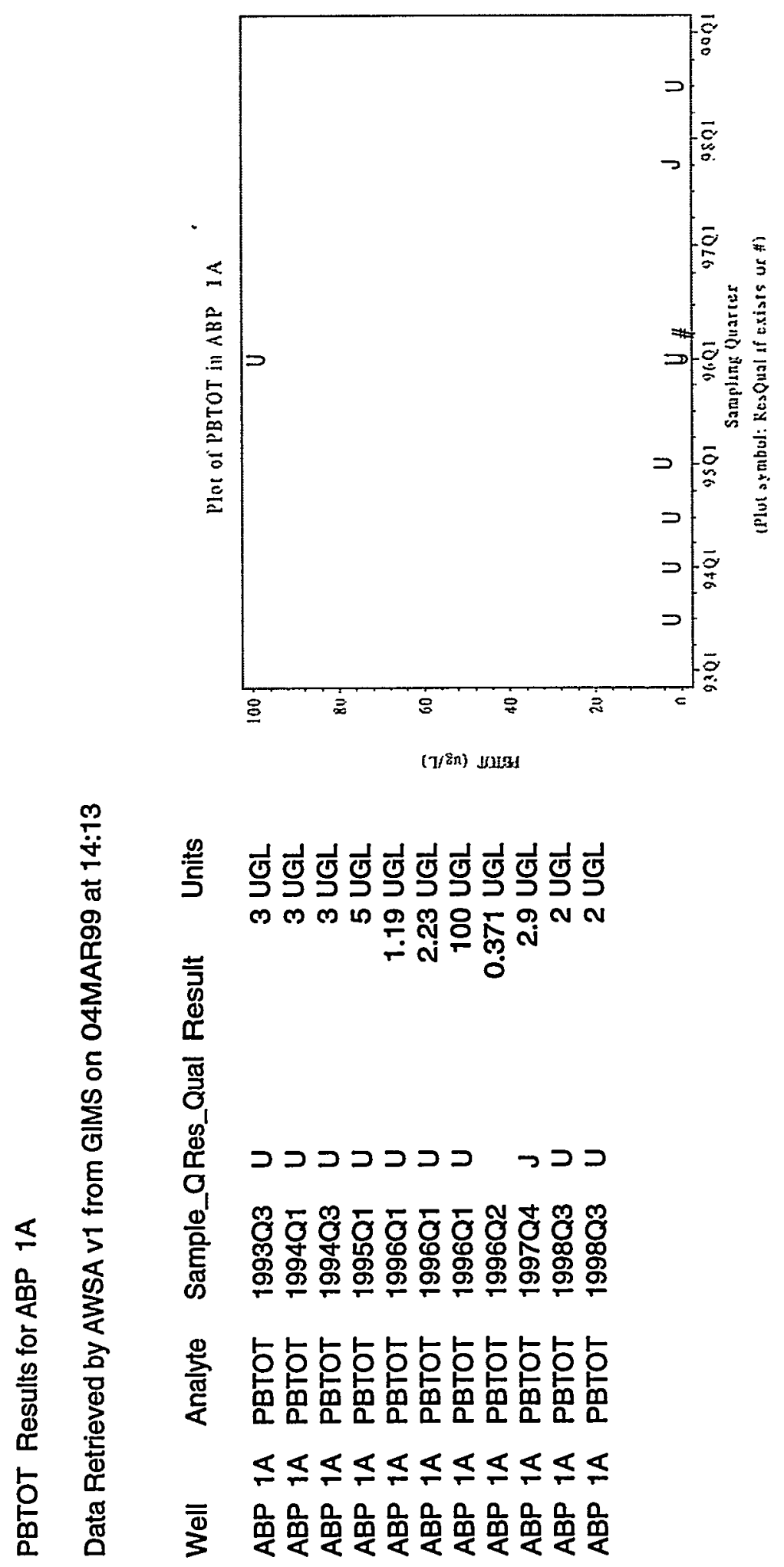
CL Results for ABP 1A

Data Retrieved by AWSA v1 from GIMS on 05MAR99 at 12:50

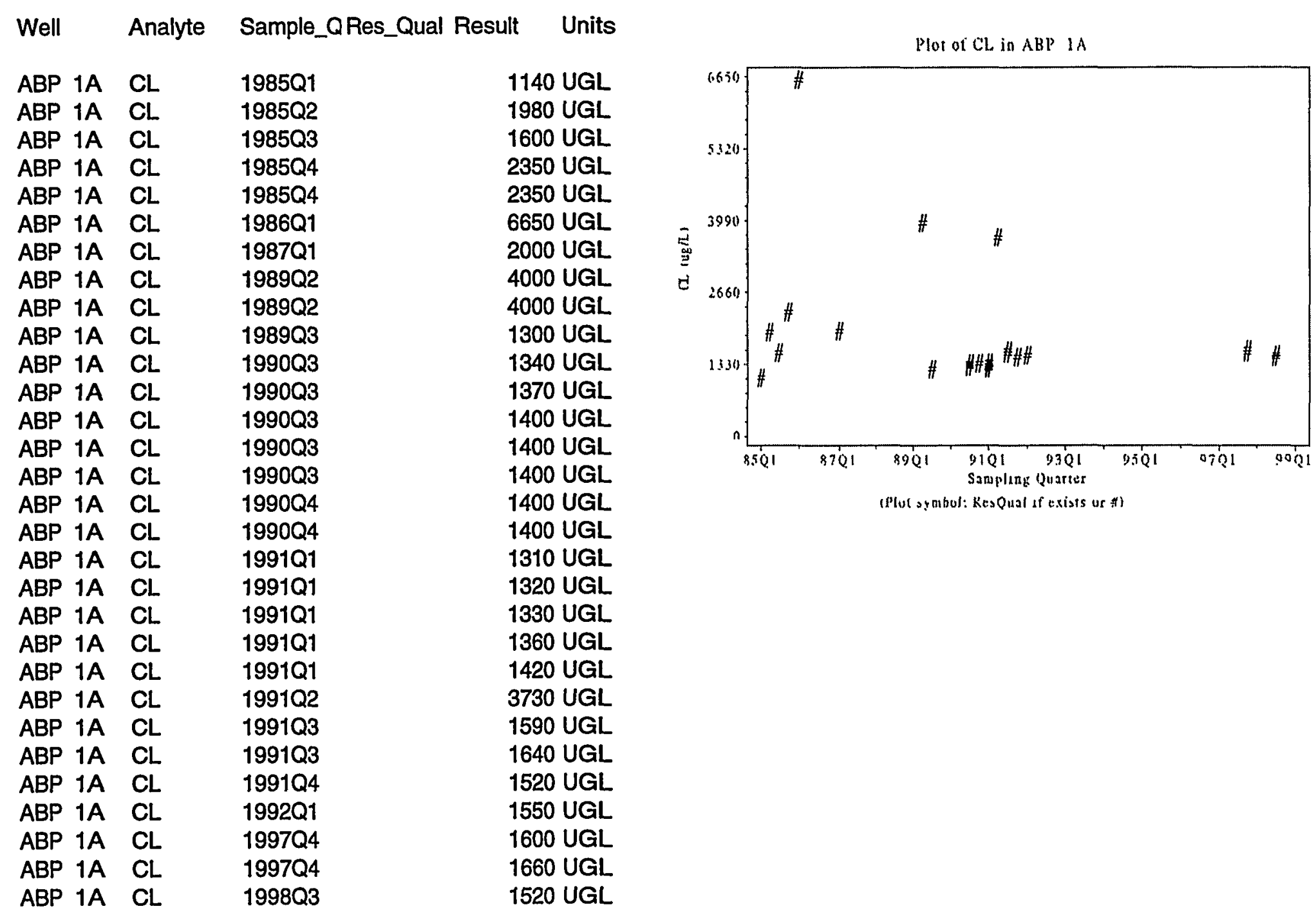


뭉
융

o

号

J

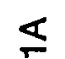

品 


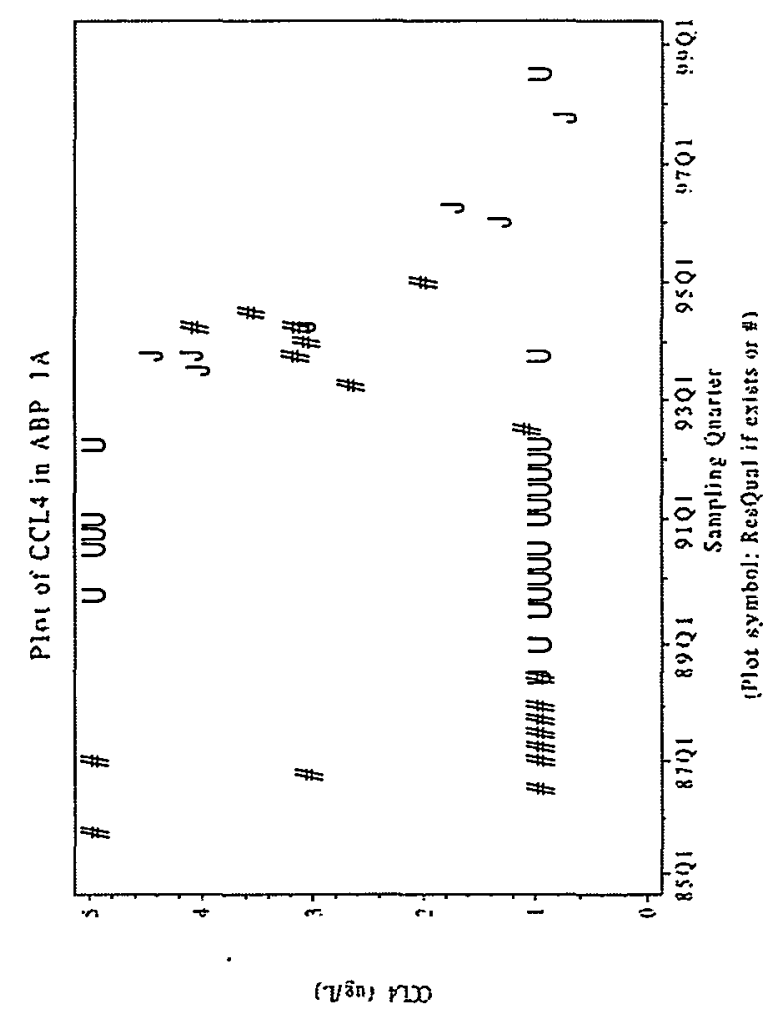

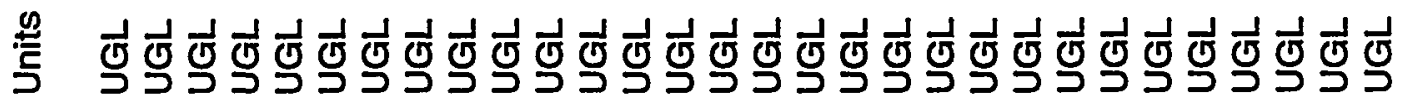
ம

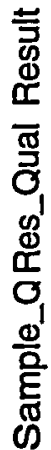

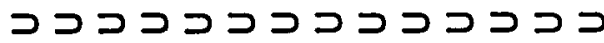

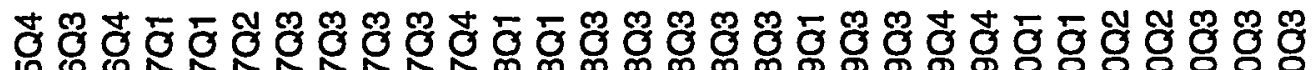

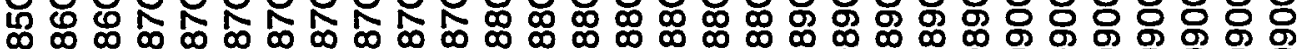

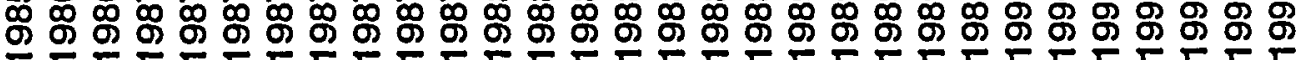

$\frac{\stackrel{9}{\frac{\pi}{\sigma}}}{\frac{\pi}{\alpha}}$

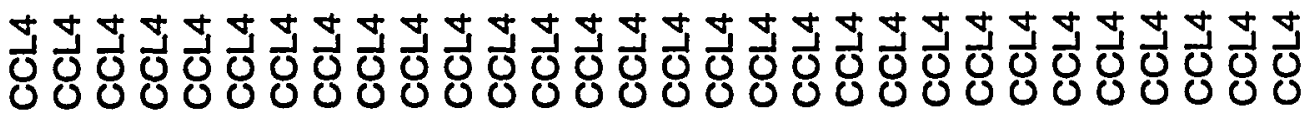

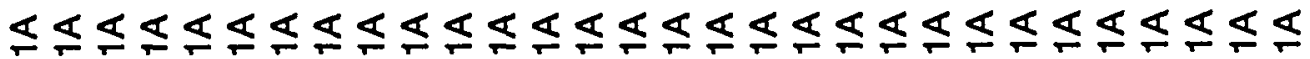

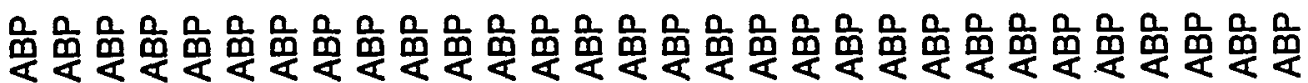




\begin{tabular}{|c|c|c|c|c|c|}
\hline ABP & $1 \mathrm{~A}$ & CCL4 & 1990Q3 & $\mathrm{U}$ & 5 UGL \\
\hline$A B P$ & $1 \mathrm{~A}$ & CCL4 & 1990Q3 & $\mathrm{u}$ & 5 UGL \\
\hline ABP & $1 \mathrm{~A}$ & CCL4 & 1990Q4 & $U$ & 5 UGL \\
\hline$A B P$ & $1 \mathrm{~A}$ & CCL4 & $1990 Q 4$ & $\mathrm{U}$ & 5 UGL \\
\hline$A B P$ & $1 \mathrm{~A}$ & CCL4 & 1991Q1 & $\mathrm{U}$ & $1 \mathrm{UGL}$ \\
\hline ABP & $1 \mathrm{~A}$ & CCL4 & 1991Q1 & $\mathbf{U}$ & 1 UGL \\
\hline ABP & $1 A$ & CCL4 & 1991Q1 & U & 1 UGL \\
\hline$A B P$ & $1 \mathrm{~A}$ & CCL4 & 1991Q1 & $\mathrm{U}$ & 5 UGL \\
\hline ABP & $1 \mathrm{~A}$ & CCL4 & 1991Q1 & $\mathrm{U}$ & 5 UGL \\
\hline ABP & $1 \mathrm{~A}$ & CCL4 & 1991Q2 & $\mathbf{U}$ & $1 \mathrm{UGL}$ \\
\hline ABP & $1 \mathrm{~A}$ & CCL4 & 1991Q3 & $U$ & 1 UGL \\
\hline ABP & $1 \mathrm{~A}$ & CCL4 & 1991Q3 & $\mathrm{U}$ & 1 UGL \\
\hline ABP & $1 \mathrm{~A}$ & CCL4 & 1991Q4 & $\mathbf{U}$ & 1 UGL \\
\hline ABP & $1 \mathrm{~A}$ & CCL4 & 1992Q1 & $\mathrm{U}$ & 1 UGI \\
\hline ABP & $1 \mathrm{~A}$ & CCL4 & 1992Q2 & $\mathrm{U}$ & $1 \mathrm{UGI}$ \\
\hline ABP & $1 \mathrm{~A}$ & CCL4 & 1992Q2 & $\mathrm{U}$ & $1 \mathrm{UG}$ \\
\hline ABP & $1 \mathrm{~A}$ & CCL4 & 1992Q2 & $U$ & $5 \mathrm{UGL}$ \\
\hline ABP & $1 \mathrm{~A}$ & CCL4 & 1992Q2 & $\mathbf{U}$ & 5 UGI \\
\hline ABP & $1 \mathrm{~A}$ & CCL4 & 1992Q3 & & 1.12 UGI \\
\hline ABP & $1 \mathrm{~A}$ & $\mathrm{CCL} 4$ & 1993Q2 & & 2.7 UGl \\
\hline ABP & $1 \mathrm{~A}$ & CCL4 & 1993Q3 & $J$ & 4.06 UG \\
\hline ABP & $1 A$ & CCL4 & 1993Q4 & & 3.2 UGI \\
\hline ABP & $1 A$ & CCL4 & 1993Q4 & $J$ & 4.12 UGL \\
\hline ABP & $1 \mathrm{~A}$ & CCL4 & 1993Q4 & $J$ & $4.48 \mathrm{UGL}$ \\
\hline ABP & $1 A$ & CCL4 & 1993Q4 & $\mathbf{U}$ & $1 U G$ \\
\hline ABP & $1 \mathrm{~A}$ & CCL4 & 1994Q1 & & $3.1 \mathrm{UG}$ \\
\hline ABP & $1 \mathrm{~A}$ & CCL4 & 1994Q2 & & $3.19 \mathrm{UG}$ \\
\hline ABP & $1 \mathrm{~A}$ & CCL4 & 1994Q2 & & 4.1 UGI \\
\hline ABP & $1 \mathrm{~A}$ & CCL4 & 1994Q2 & $J$ & 3.11 UG \\
\hline ABP & $1 \mathrm{~A}$ & $\mathrm{CCL} 4$ & 1994Q2 & $\mathbf{J}$ & $3.16 \mathrm{UG}$ \\
\hline ABP & $1 \mathrm{~A}$ & $\mathrm{CCL}_{4} 4$ & 1994Q3 & & $3.6 \mathrm{UG}$ \\
\hline ABP & $1 A$ & CCL4 & 1995Q1 & & 2.05 UG \\
\hline ABP & $1 \mathrm{~A}$ & CCL4 & 1996Q1 & $J$ & $1.36 \mathrm{UG}$ \\
\hline ABP & $1 \mathrm{~A}$ & CCL4 & 1996Q2 & $\mathrm{J}$ & $1.78 \mathrm{UGl}$ \\
\hline$A B P$ & $1 \mathrm{~A}$ & $\mathrm{CCL} 4$ & 1997Q4 & $J$ & 0.783 UGL \\
\hline ABP & $1 \mathrm{~A}$ & CCL4 & 1998Q3 & $\mathbf{U}$ & 1 UGI \\
\hline ABP & $1 \mathrm{~A}$ & CCL4 & 1998Q3 & $U$ & 1 UG \\
\hline
\end{tabular}


TCLEE Results for ABP 1A

Data Retrieved by AWSA v1 from GIMS on 04MAR99 at 14:13

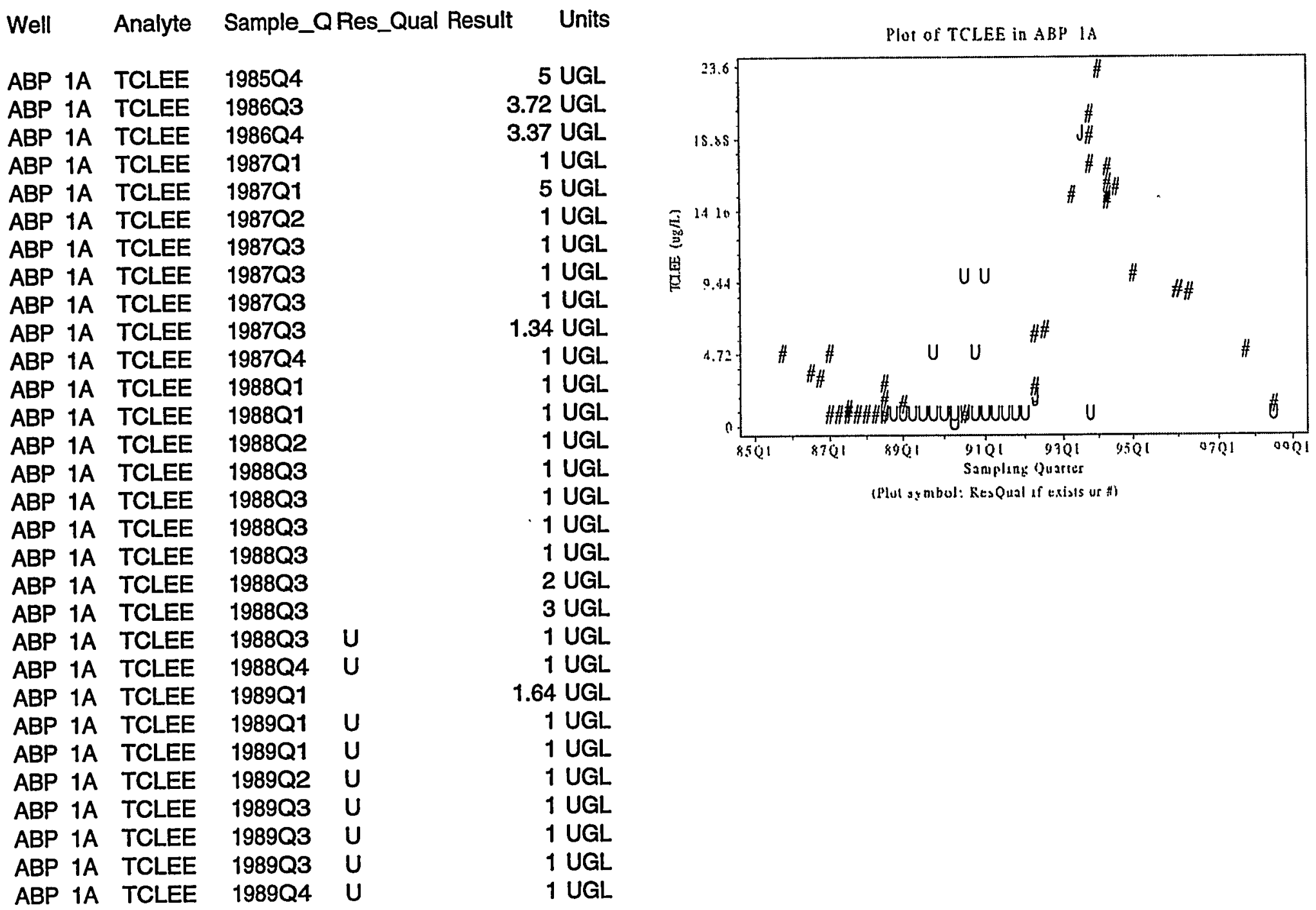




$\begin{array}{llll}\text { ABP 1A } & \text { TCLEE } & 1989 Q 4 & U \\ \text { ABP 1A } & \text { TCLEE } & 1989 Q 4 & U \\ \text { ABP 1A } & \text { TCLEE } & 1989 Q 4 & U \\ \text { ABP 1A } & \text { TCLEE } & 1990 Q 1 & U \\ \text { ABP 1A } & \text { TCLEE } & 1990 Q 1 & U \\ \text { ABP 1A } & \text { TCLEE } & 1990 Q 1 & U \\ \text { ABP 1A } & \text { TCLEE } & 1990 Q 2 & U \\ \text { ABP 1A } & \text { TCLEE } & 1990 Q 2 & U \\ \text { ABP 1A } & \text { TCLEE } & 1990 Q 2 & U \\ \text { ABP 1A } & \text { TCLEE } & 1990 Q 2 & U \\ \text { ABP 1A } & \text { TCLEE } & 1990 Q 3 & \\ \text { ABP 1A } & \text { TCLEE } & 1990 Q 3 & U \\ \text { ABP 1A } & \text { TCLEE } & 1990 Q 3 & U \\ \text { ABP 1A } & \text { TCLEE } & 1990 Q 3 & U \\ \text { ABP 1A } & \text { TCLEE } & 1990 Q 3 & U \\ \text { ABP 1A } & \text { TCLEE } & 1990 Q 3 & U \\ \text { ABP 1A } & \text { TCLEE } & 1990 Q 3 & U \\ \text { ABP 1A } & \text { TCLEE } & 1990 Q 4 & U \\ \text { ABP 1A } & \text { TCLEE } & 1990 Q 4 & U \\ \text { ABP 1A } & \text { TCLEE } & 1990 Q 4 & U \\ \text { ABP 1A } & \text { TCLEE } & 1991 Q 1 & U \\ \text { ABP 1A } & \text { TCLEE } & 1991 Q 1 & U \\ \text { ABP 1A } & \text { TCLEE } & 1991 Q 1 & U \\ \text { ABP 1A } & \text { TCLEE } & 1991 Q 1 & U \\ \text { ABP 1A } & \text { TCLEE } & 1991 Q 1 & U \\ \text { ABP 1A } & \text { TCLEE } & 1991 Q 2 & U \\ \text { ABP 1A } & \text { TCLEE } & 1991 Q 3 & U \\ \text { ABP 1A } & \text { TCLEE } & 1991 Q 3 & U \\ \text { ABP 1A } & \text { TCLEE } & 1991 Q 4 & U \\ \text { ABP 1A } & \text { TCLEE } & 1992 Q 1 & U \\ \text { ABP 1A } & \text { TCLEE } & 1992 Q 2 & \\ \text { ABP 1A } & \text { TCLEE } & 1992 Q 2 & \\ \text { ABP 1A } & \text { TCLEE } & 1992 Q 2 & \mathrm{~J} \\ \text { ABP 1A } & \text { TCLEE } & 1992 Q 2 & \mathrm{~J} \\ \text { ABP 1A } & \text { TCLEE } & 1992 Q 3 & \\ \text { ABP 1A } & \text { TCLEE } & 1993 Q 2 & \\ \text { ABP 1A } & \text { TCLEE } & 1993 Q 3 & \mathrm{~J}\end{array}$

1 UGL

1 UGL

5 UGL

1 UGL

1 UGL

1 UGL

0.4 UGL

1 UGL

1 UGL

1 UGL

1 UGL

1 UGL

1 UGL

1 UGL

1 UGL

10 UGL

10 UGL

1 UGL

5 UGL

5 UGL

1 UGL

1 UGL

1 UGL

10 UGL

10 UGL

1 UGL

1 UGL

1 UGL

$1 \mathrm{UGL}$

1 UGL

2.77 UGL

6.23 UGL

1.97 UGL

$2.16 \mathrm{UGL}$

6.52 UGL

15.4 UGL

19.4 UGL 


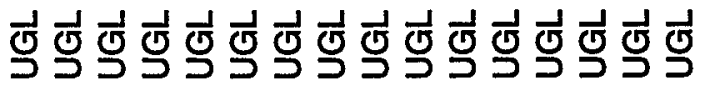

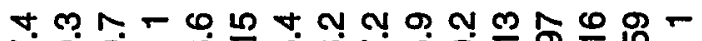
둉

$>>$

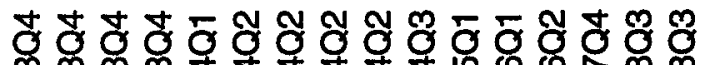

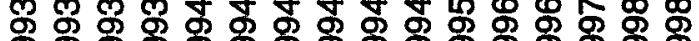

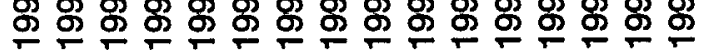

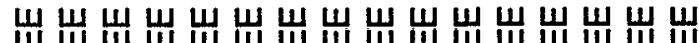
Ð

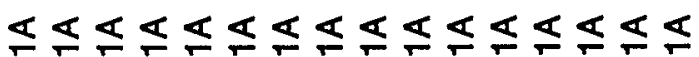

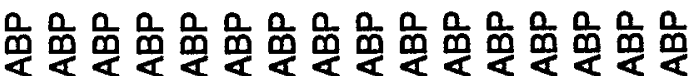


TRCLE Results for ABP 1A

Data Retrieved by AWSA v1 from GIMS on 04MAR99 at 14:13

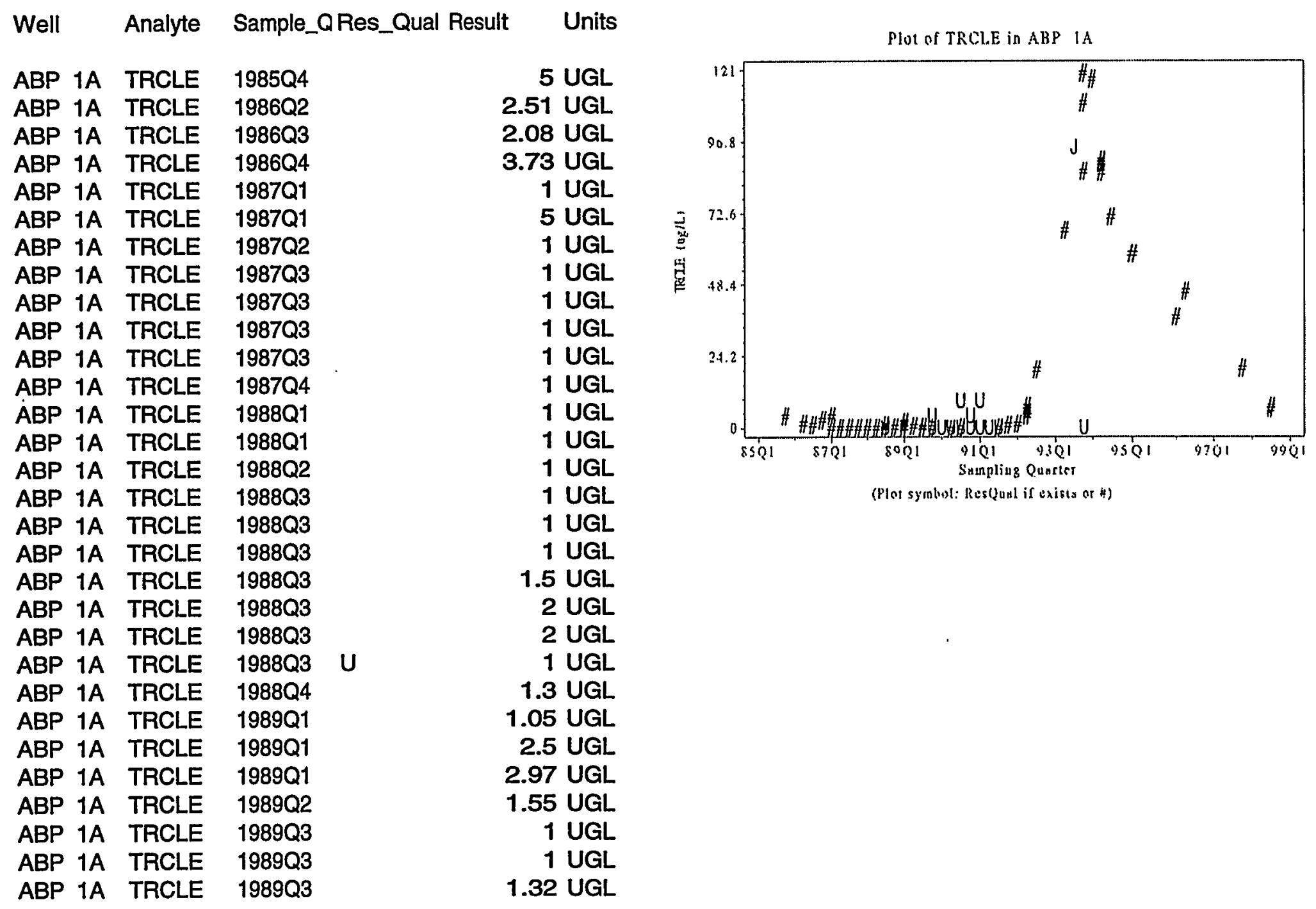




\begin{tabular}{|c|c|c|c|c|}
\hline$A B P \quad 1 A$ & TRCLE & 1989Q4 & & 1.07 UGL \\
\hline$A B P \quad 1 A$ & TRCLE & 1989Q4 & $U$ & $1 \mathrm{UGL}$ \\
\hline$A B P \quad 1 A$ & TRCLE & 1989Q4 & $U$ & $1 \mathrm{UGL}$ \\
\hline$A B P 1 A$ & TRCLE & 1989Q4 & $U$ & 5 UGL \\
\hline$A B P 1 A$ & TRCLE & 1990Q1 & $\mathrm{U}$ & $1 \mathrm{UGL}$ \\
\hline$A B P \quad 1 A$ & TRCLE & 1990Q1 & $U$ & $1 \mathrm{UGL}$ \\
\hline$A B P 1 A$ & TRCLE & 1990Q1 & $U$ & $1 \mathrm{UGL}$ \\
\hline$A B P \quad 1 A$ & TRCLE & 1990Q2 & & 0.58 UGL \\
\hline$A B P \quad 1 A$ & TRCLE & 1990Q2 & $U$ & $1 \mathrm{UGL}$ \\
\hline$A B P \quad 1 A$ & TRCLE & 1990Q2 & $\mathrm{U}$ & 1 UGL \\
\hline$A B P 1 A$ & TRCLE & 1990Q2 & $U$ & $1 \mathrm{UGL}$ \\
\hline$A B P \quad 1 A$ & TRCLE & 1990Q3 & & 1 UGL \\
\hline$A B P \quad 1 A$ & TRCLE & 1990Q3 & & $1 \mathrm{UGL}$ \\
\hline ABP IA & TRCLE & 1990Q3 & & $1.15 \mathrm{UGL}$ \\
\hline$A B P 1 A$ & TRCLE & 1990Q3 & U & 1 UGL \\
\hline ABP $1 A$ & TRCLE & 1990Q3 & $U$ & $1 \mathrm{UGL}$ \\
\hline$A B P 1 A$ & TRCLE & 1990Q3 & $U$ & 10 UGL \\
\hline ABP $1 A$ & TRCLE & 1990Q3 & $U$ & 10 UGL \\
\hline$A B P \quad 1 A$ & TRCLE & 1990Q4 & $\mathrm{U}$ & 1 UGL \\
\hline$A B P 1 A$ & TRCLE & 1990Q4 & $\mathbf{U}$ & 5 UGL \\
\hline$A B P \quad 1 A$ & TRCLE & $1990 Q 4$ & $\mathbf{U}$ & 5 UGL \\
\hline$A B P \quad 1 A$ & TRCLE & 1991Q1 & $\mathbf{U}$ & 1 UGL \\
\hline$A B P \quad 1 A$ & TRCLE & 1991Q1 & $\mathbf{U}$ & $1 \mathrm{UGL}$ \\
\hline ABP $1 A$ & TRCLE & 1991Q1 & $\mathbf{U}$ & $1 \mathrm{UGL}$ \\
\hline ABP $1 A$ & TRCLE & 1991Q1 & $\mathbf{U}$ & 10 UGL \\
\hline$A B P \quad 1 A$ & TRCLE & 1991Q1 & $\mathbf{U}$ & 10 UGL \\
\hline ABP $1 A$ & TRCLE & 1991Q2 & $\mathbf{U}$ & $1 \mathrm{UGL}$ \\
\hline$A B P \quad 1 A$ & TRCLE & 1991Q3 & & 1 UGL \\
\hline$A B P \quad 1 A$ & TRCLE & 1991Q3 & $U$ & $1 \mathrm{UGL}$ \\
\hline$A B P 1 A$ & TRCLE & 1991Q4 & & 2 UGL \\
\hline$A B P \quad 1 A$ & TRCLE & 1992Q1 & & 2.29 UGL \\
\hline$A B P 1 A$ & TRCLE & 1992Q2 & & 5.05 UGL \\
\hline$A B P \quad 1 A$ & TRCLE & 1992Q2 & & 5.37 UGL \\
\hline ABP $1 A$ & TRCLE & 1992Q2 & & 6.09 UGL \\
\hline$A B P \quad 1 A$ & TRCLE & 1992Q2 & & 8.28 UGL \\
\hline$A B P \quad 1 A$ & TRCLE & 1992Q3 & & 20.7 UGL \\
\hline ABP 1 & TRCLE & 1993Q2 & & 67.9 UGL \\
\hline
\end{tabular}




\begin{tabular}{|c|c|c|c|c|}
\hline$A B P 1 A$ & TRCLE & 1993Q3 & $J$ & 95.9 UGL \\
\hline$A B P 1 A$ & TRCLE & 1993Q4 & & 87.8 UGL \\
\hline ABP $1 \mathrm{~A}$ & TRCLE & 1993Q4 & & 111 UGL \\
\hline$A B P 1 A$ & TRCLE & 1993Q4 & & 121 UGL \\
\hline$A B P$ IA & TRCLE & 1993Q4 & $u$ & 1 UGL \\
\hline$A B P \quad 1 A$ & TRCLE & 1994Q1 & & 119 UGL \\
\hline$A B P 1 A$ & TRCLE & 1994Q2 & & 87.5 UGL \\
\hline$A B P 1 A$ & TRCLE & 1994Q2 & & 90.5 UGL \\
\hline$A B P 1 A$ & TRCLE & 1994Q2 & & 90.9 UGL \\
\hline ABP $1 A$ & TRCLE & 1994Q2 & & $91.6 \mathrm{UGL}$ \\
\hline$A B P \quad 1 A$ & TRCLE & 1994Q3 & & 72.4 UGL \\
\hline$A B P 1 A$ & TRCLE & 1995Q1 & & 59.9 UGL \\
\hline ABP 1A & TRCLE & 1996Q1 & & 38.4 UGL \\
\hline$A B P 1 A$ & TRCLE & 1996Q2 & & 47.2 UGL \\
\hline$A B P 1 A$ & TRCLE & 1997Q4 & & 21.1 UGL \\
\hline$A B P 1 A$ & TRCLE & 1998Q3 & & 7.24 UGL \\
\hline$A B P$ 1A & TRCLE & 1998Q3 & & 8.28 UGI \\
\hline
\end{tabular}


ALPHAG Results for ABP $1 \mathrm{~A}$

Data Retrieved by AWSA v1 from GIMS on 04MAR99 at 14:13

Well Analyte Sample_QRes_Qual Result Units

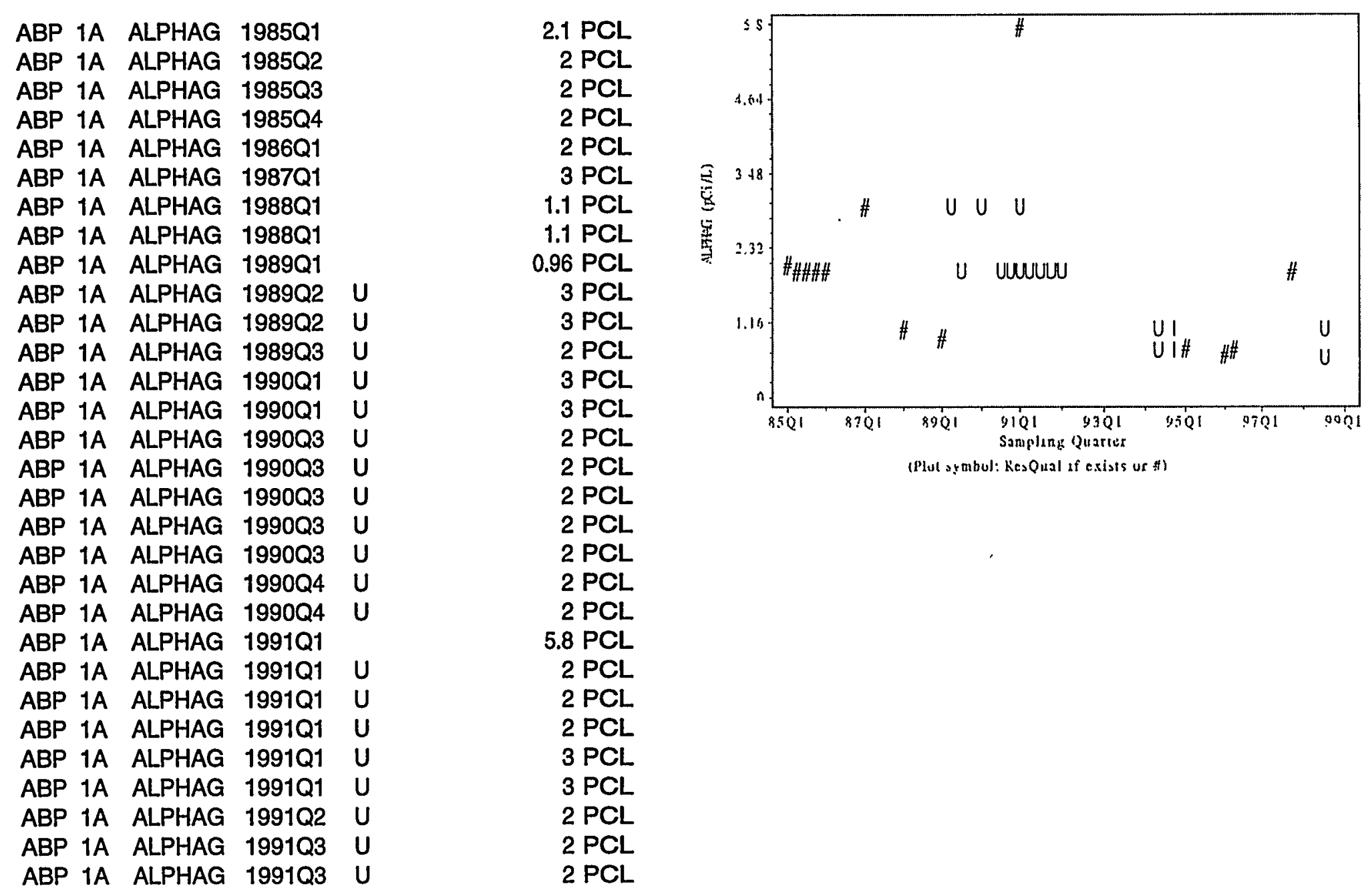




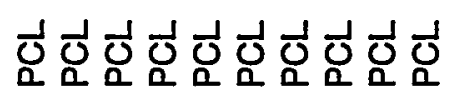

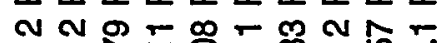

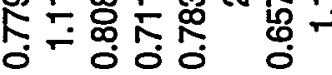

つ つ

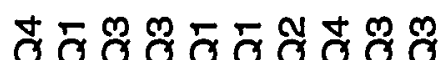

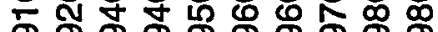

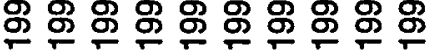

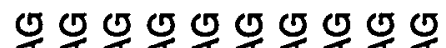

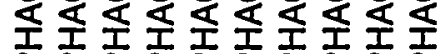

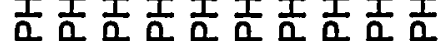

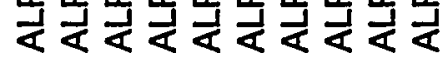
$\leqslant \leq \leqslant \leq \leqslant \leq \leqslant \leq \leqslant \leq$

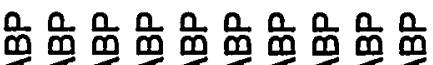

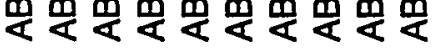


WELL ABP-4 DATA 


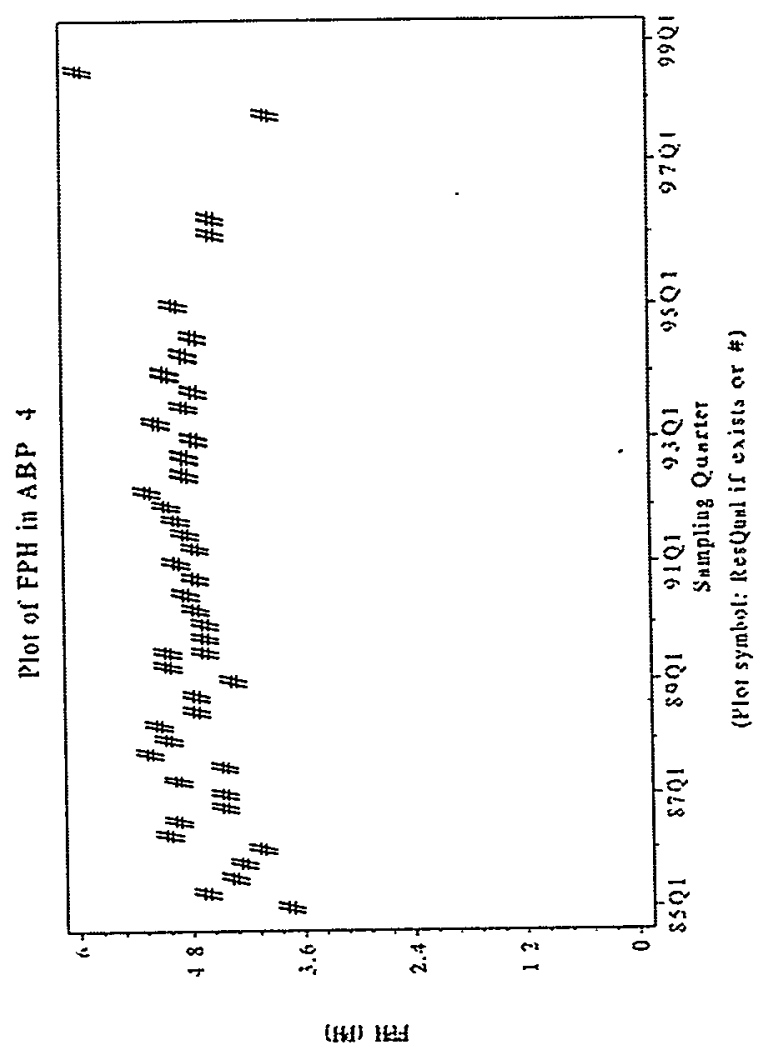

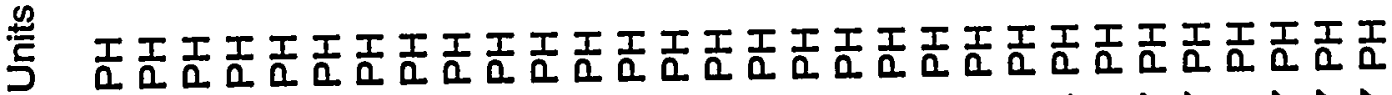

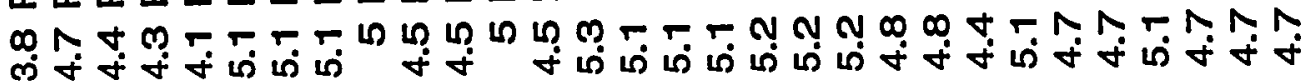

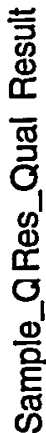

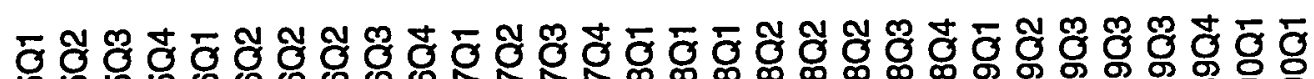

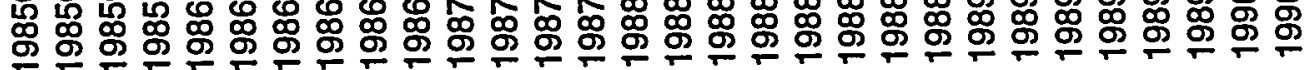

$\frac{\mathscr{Q}}{\frac{\pi}{\sigma}}$

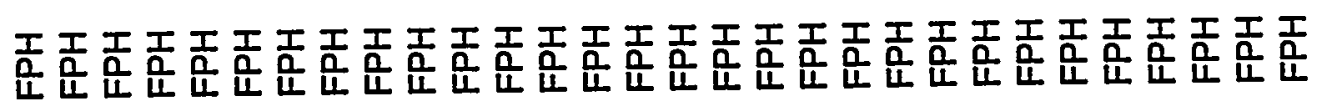

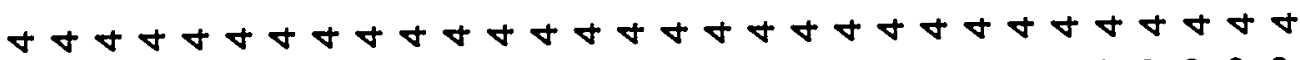

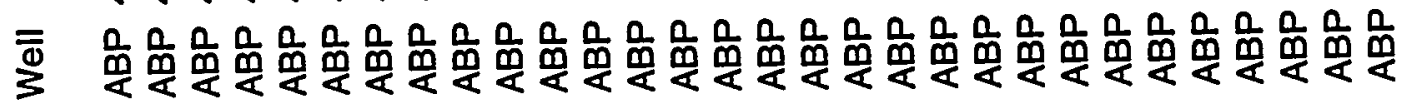




$\begin{array}{lllr}\text { ABP 4 } & \text { FPH } & 1990 Q 2 & 4.8 \mathrm{PH} \\ \text { ABP 4 } & \text { FPH } & 1990 Q 3 & 4.9 \mathrm{PH} \\ \text { ABP 4 } & \text { FPH } & 1990 Q 4 & 4.8 \mathrm{PH} \\ \text { ABP 4 } & \text { FPH } & 1990 Q 4 & 4.8 \mathrm{PH} \\ \text { ABP 4 } & \text { FPH } & 1991 Q 1 & 5 \mathrm{PH} \\ \text { ABP 4 } & \text { FPH } & 1991 Q 2 & 4.8 \mathrm{PH} \\ \text { ABP 4 } & \text { FPH } & 1991 Q 3 & 4.9 \mathrm{PH} \\ \text { ABP 4 } & \text { FPH } & 1991 Q 4 & 5 \mathrm{PH} \\ \text { ABP 4 } & \text { FPH } & 1992 Q 1 & 5.1 \mathrm{PH} \\ \text { ABP 4 } & \text { FPH } & 1992 Q 1 & 5.1 \mathrm{PH} \\ \text { ABP 4 } & \text { FPH } & 1992 Q 2 & 5.3 \mathrm{PH} \\ \text { ABP 4 } & \text { FPH } & 1992 Q 2 & 5.3 \mathrm{PH} \\ \text { ABP 4 } & \text { FPH } & 1992 Q 3 & 4.9 \mathrm{PH} \\ \text { ABP 4 } & \text { FPH } & 1992 Q 4 & 4.9 \mathrm{PH} \\ \text { ABP 4 } & \text { FPH } & 1993 Q 1 & 4.8 \mathrm{PH} \\ \text { ABP 4 } & \text { FPH } & 1993 Q 2 & 5.2 \mathrm{PH} \\ \text { ABP 4 } & \text { FPH } & 1993 Q 3 & 4.9 \mathrm{PH} \\ \text { ABP 4 } & \text { FPH } & 1993 Q 4 & 4.8 \mathrm{PH} \\ \text { ABP 4 } & \text { FPH } & 1994 Q 1 & 5.1 \mathrm{PH} \\ \text { ABP 4 } & \text { FPH } & 1994 Q 2 & 4.9 \mathrm{PH} \\ \text { ABP 4 } & \text { FPH } & 1994 Q 3 & 4.8 \mathrm{PH} \\ \text { ABP 4 } & \text { FPH } & 1995 Q 1 & 5 \mathrm{PH} \\ \text { ABP 4 } & \text { FPH } & 1996 Q 1 & 4.6 \mathrm{PH} \\ \text { ABP 4 } & \text { FPH } & 1996 Q 2 & 4.6 \mathrm{PH} \\ \text { ABP 4 } & \text { FPH } & 1997 Q 4 & 4 \mathrm{PH} \\ \text { ABP 4 } & \text { FPH } & 1998 Q 3 & 6 \mathrm{PH} \\ \text { ABP 4 } & \text { FPH } & 1998 Q 3 & 6 \mathrm{PH}\end{array}$


COND Results for ABP 4

Data Retrieved by AWSA v1 from GIMS on 04MAR99 at 10:55

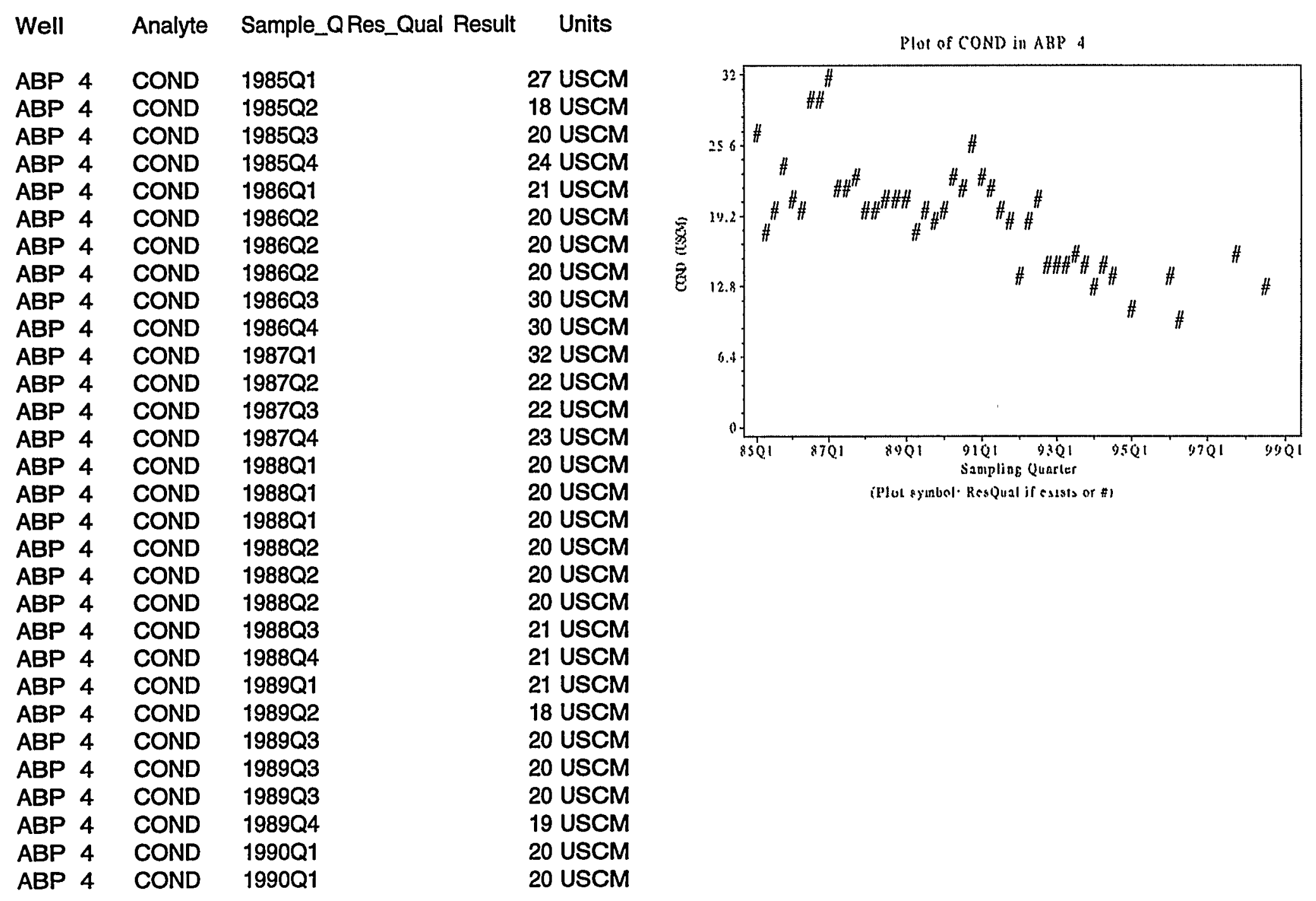




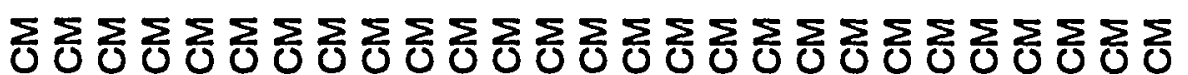
DS

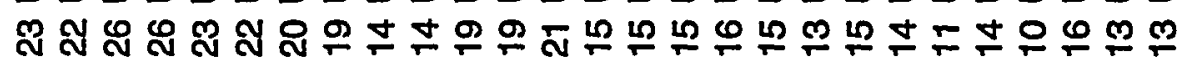

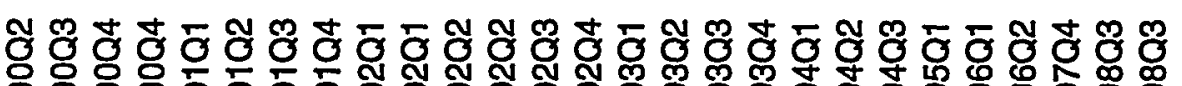

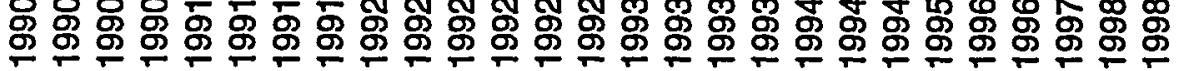

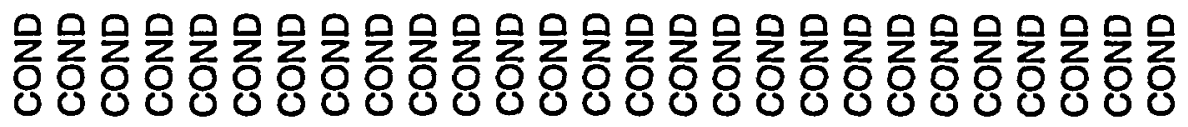

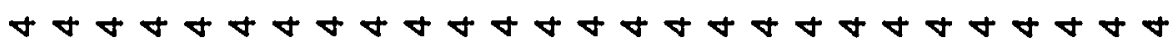

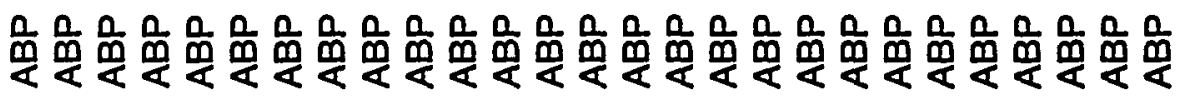


CATOT Results for ABP 4

Data Retrieved by AWSA v1 from GIMS on 04MAR99 at 14:13

\begin{tabular}{|c|c|c|c|c|}
\hline Well & Analyte & Sample_C & 2Res_Qual Resul & Units \\
\hline ABP 4 & САTOT & 1993Q1 & & 540 UGL \\
\hline ABP 4 & CATOT & 1993Q1 & & 599 UGL \\
\hline ABP 4 & САTOT & 1993Q1 & & 615 UGL \\
\hline ABP 4 & CATOT & 1993Q1 & & 634 UGL \\
\hline ABP 4 & САTOT & 1993Q1 & & 637 UGL \\
\hline ABP 4 & CATOT & 1996Q1 & & 503 UGL \\
\hline ABP 4 & CATOT & 1996Q1 & & 560 UGL \\
\hline ABP 4 & САTOT & 1996Q1 & $J$ & 399 UGL \\
\hline ABP 4 & CATOT & 1996Q1 & J & 425 UGL \\
\hline ABP 4 & СATOT & 1996Q1 & J & 426 UGL \\
\hline ABP 4 & САTOT & 1996Q2 & & 557 UGL \\
\hline ABP 4 & CATOT & 1997Q4 & & 477 UGL \\
\hline ABP 4 & CATOT & 1998Q3 & & 543 UGL \\
\hline ABP 4 & СATOT & 1998Q3 & & 645 UGL \\
\hline
\end{tabular}

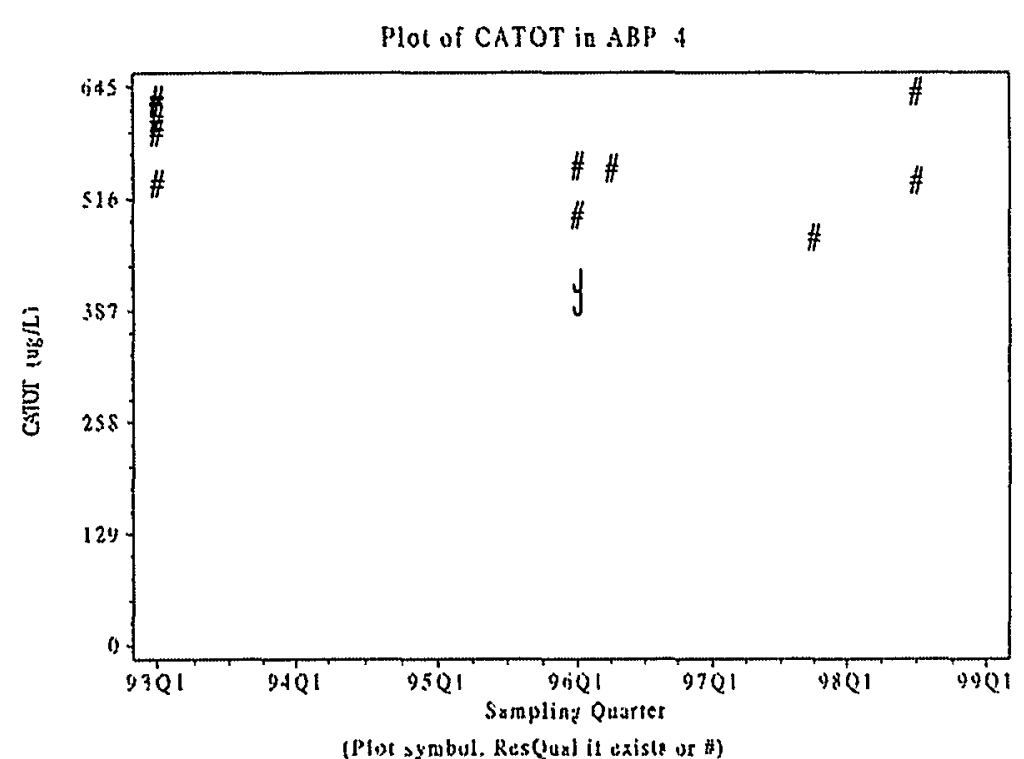


KTOT Results for ABP 4

Data Retrieved by AWSA v1 from GIMS on 04MAR99 at 14:13

\begin{tabular}{llll} 
Well & Analyte & \multicolumn{2}{c}{ Sample_QRes_Q } \\
& & & \\
ABP 4 & KTOT & $1993 Q 1$ & $\mathrm{~J}$ \\
ABP 4 & KTOT & $1993 Q 1$ & $\mathrm{U}$ \\
ABP 4 & KTOT & $1993 Q 1$ & $\mathrm{U}$ \\
ABP 4 & KTOT & $1993 Q 1$ & $\mathrm{U}$ \\
ABP 4 & KTOT & $1993 Q 1$ & $\mathrm{U}$ \\
ABP 4 & KTOT & $1996 Q 1$ & \\
ABP 4 & KTOT & $1996 Q 1$ & \\
ABP 4 & KTOT & $1996 Q 1$ & \\
ABP 4 & KTOT & $1996 Q 1$ & $\mathrm{~J}$ \\
ABP 4 & KTOT & $1996 Q 1$ & $\mathrm{~J}$ \\
ABP 4 & KTOT & $1996 Q 2$ & $\mathrm{U}$ \\
ABP 4 & KTOT & $1997 Q 4$ & \\
ABP 4 & KTOT & $1998 Q 3$ & \\
ABP 4 & KTOT & $1998 Q 3$ &
\end{tabular}

Units

519 UGL $500 \mathrm{UGL}$ 500 UGL 500 UGL 500 UGL 148 UGL 171 UGL 188 UGL 189 UGL 192 UGL 2000 UGL 177 UGL 193 UGL 196 UGL

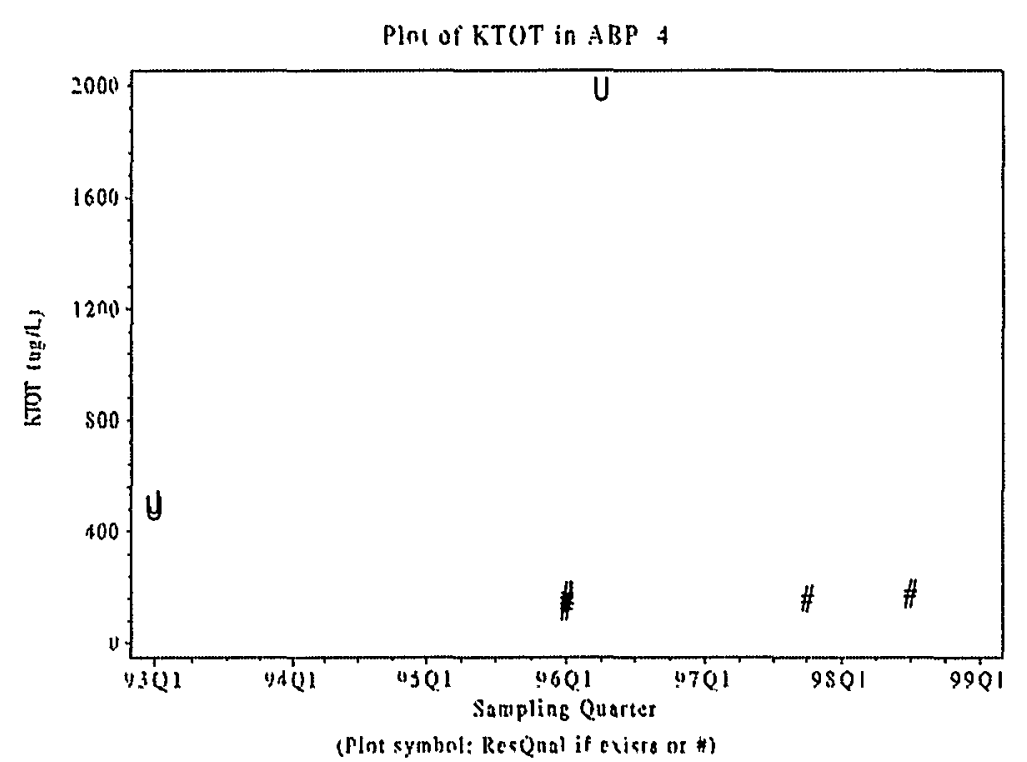


NATOT Results for ABP 4

Data Retrieved by AWSA v1 from GIMS on 04MAR99 at 14:13

$\begin{array}{llrr}\text { Well } & \text { Analyte } & \text { Sample_QRes_Qual Result . Units } \\ & & & \\ \text { ABP 4 } & \text { NATOT } & 1993 Q 1 & 851 \text { UGL } \\ \text { ABP 4 } & \text { NATOT } & 1993 Q 1 & 877 \text { UGL } \\ \text { ABP 4 } & \text { NATOT } & 1993 Q 1 & 881 \text { UGL } \\ \text { ABP 4 } & \text { NATOT } & 1993 Q 1 & 923 \text { UGL } \\ \text { ABP 4 } & \text { NATOT } & 1993 Q 1 & 938 \text { UGL } \\ \text { ABP 4 } & \text { NATOT } & 1996 Q 1 & 765 \text { UGL } \\ \text { ABP 4 } & \text { NATOT } & 1996 Q 1 & 812 \text { UGL } \\ \text { ABP 4 } & \text { NATOT } & 1996 Q 1 & 813 \text { UGL } \\ \text { ABP 4 } & \text { NATOT } & 1996 Q 1 & 900 \text { UGL } \\ \text { ABP 4 } & \text { NATOT } & 1996 Q 1 & 905 \text { UGL } \\ \text { ABP 4 } & \text { NATOT } & 1996 Q 2 & 1110 \text { UGL } \\ \text { ABP 4 } & \text { NATOT } & 1997 Q 4 & 946 \text { UGL } \\ \text { ABP 4 } & \text { NATOT } & 1998 Q 3 & 931 \text { UGL } \\ \text { ABP 4 } & \text { NATOT } & 1998 Q 3 & 945 \text { UGL }\end{array}$

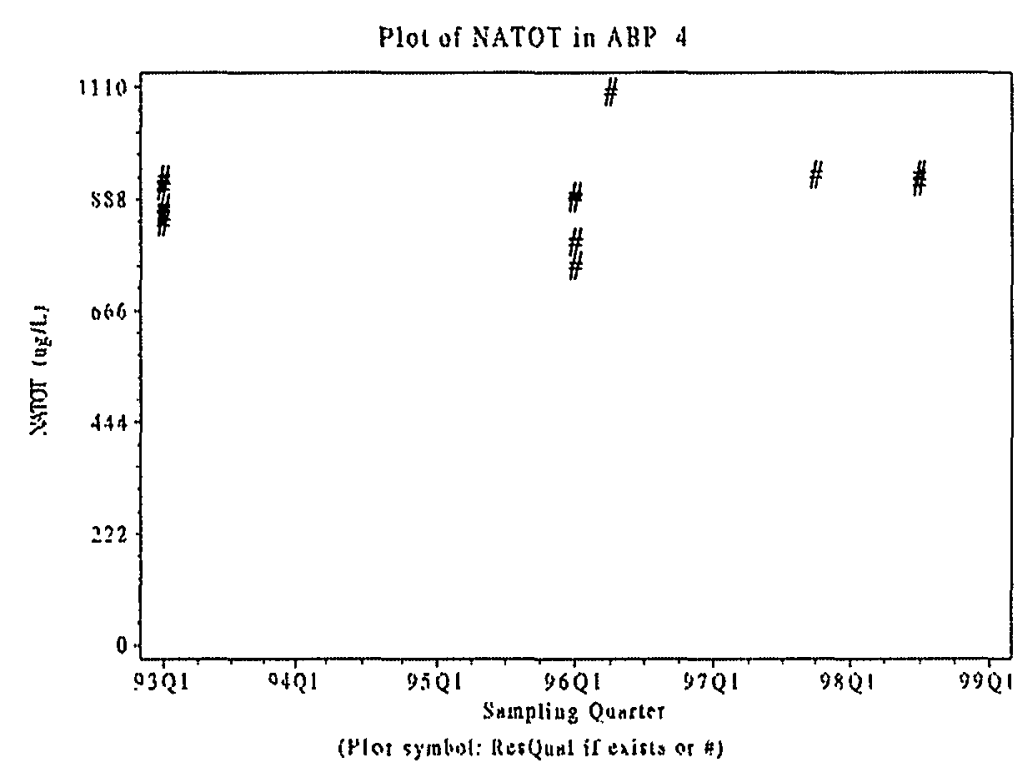



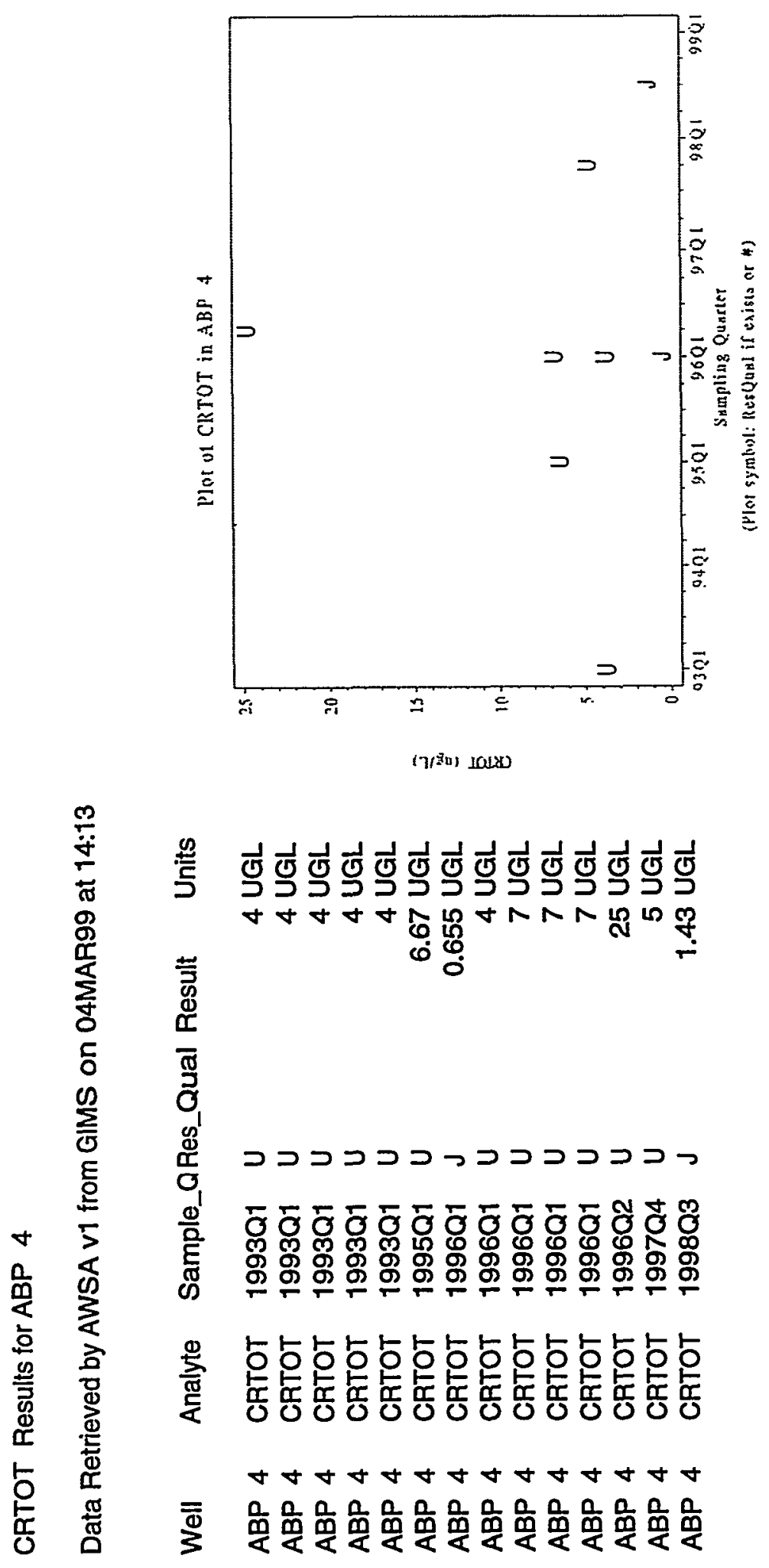

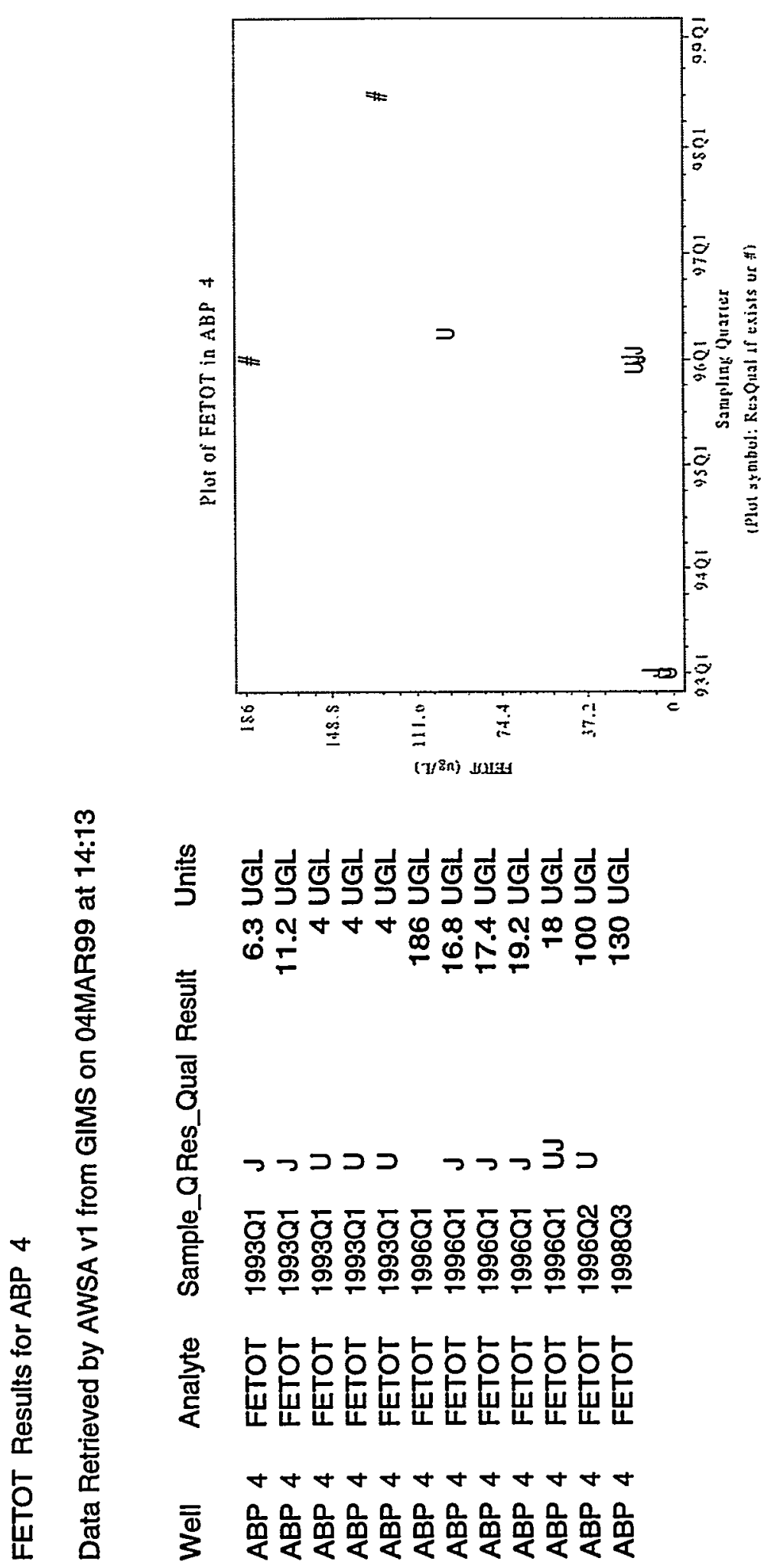


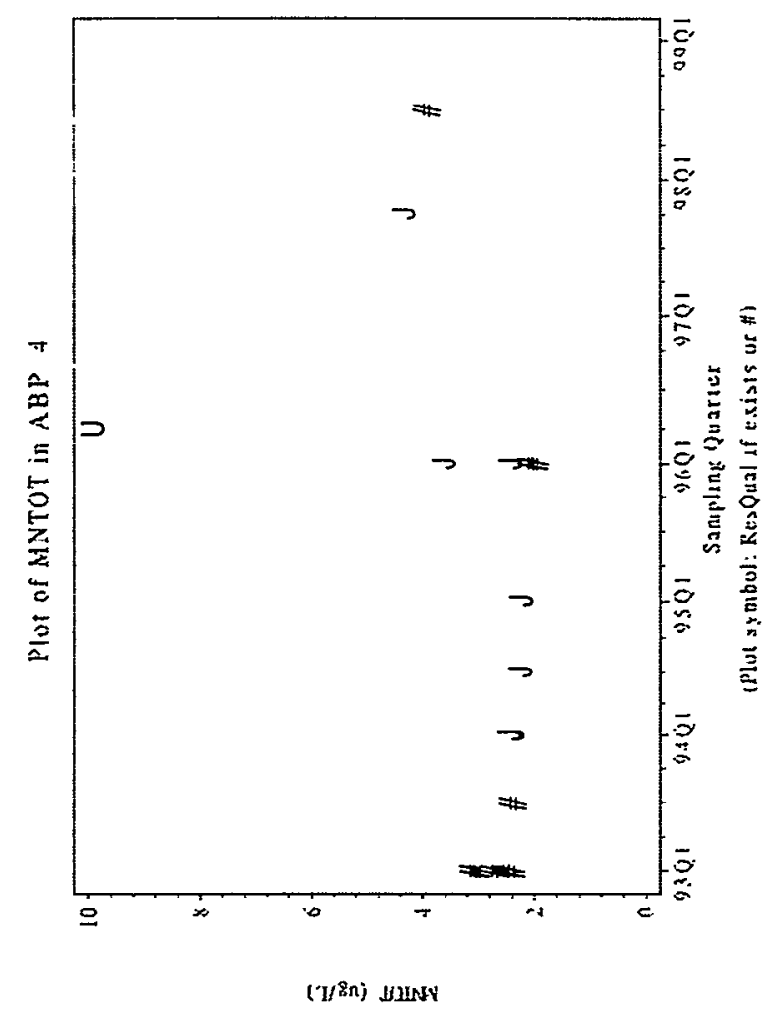

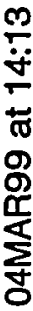

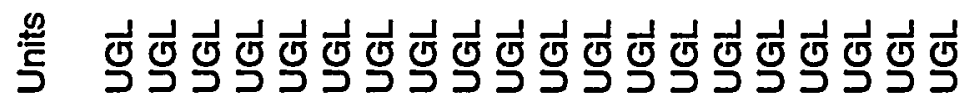
ஸी

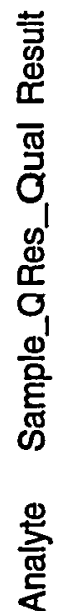
つつつつつ つつつつつ

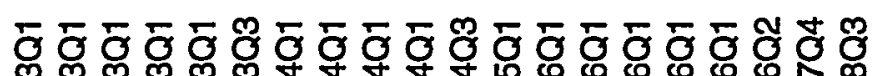
䨗

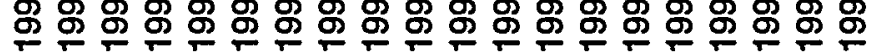

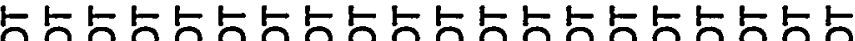

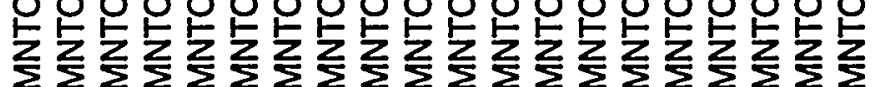


ALTOT Results for ABP 4

Data Retrieved by AWSA v1 from GIMS on 04MAR99 at 14:13

\begin{tabular}{llllr} 
Well & Analyte & \multicolumn{2}{c}{ Sample_QRes_Qual Result } & Units \\
& & & & \\
ABP 4 & ALTOT & $1992 Q 4$ & $U$ & 20 UGL \\
ABP 4 & ALTOT & $1993 Q 1$ & $U$ & 20 UGL \\
ABP 4 & ALTOT & $1993 Q 1$ & $U$ & 20 UGL \\
ABP 4 & ALTOT & $1993 Q 1$ & $U$ & 20 UGL \\
ABP 4 & ALTOT & $1993 Q 1$ & $U$ & 20 UGL \\
ABP 4 & ALTOT & $1993 Q 1$ & $U$ & 20 UGL \\
ABP 4 & ALTOT & $1993 Q 3$ & $U$ & 20 UGL \\
ABP 4 & ALTOT & $1994 Q 1$ & $U$ & 20 UGL \\
ABP 4 & ALTOT & $1994 Q 1$ & $U$ & 20 UGL \\
ABP 4 & ALTOT & $1994 Q 1$ & $U$ & 20 UGL \\
ABP 4 & ALTOT & $1994 Q 1$ & $U$ & 20 UGL \\
ABP 4 & ALTOT & $1994 Q 3$ & $U$ & 20 UGL \\
ABP 4 & ALTOT & $1995 Q 1$ & $U$ & 33.3 UGL \\
ABP 4 & ALTOT & $1996 Q 1$ & $U$ & 14.8 UGL \\
ABP 4 & ALTOT & $1996 Q 1$ & $U$ & 21.1 UGL \\
ABP 4 & ALTOT & $1996 Q 1$ & $U$ & 146 UGL \\
ABP 4 & ALTOT & $1996 Q 1$ & $U$ & 146 UGL \\
ABP 4 & ALTOT & $1996 Q 1$ & $U$ & 146 UGL \\
ABP 4 & ALTOT & $1996 Q 2$ & $U$ & 250 UGL \\
ABP 4 & ALTOT & $1997 Q 4$ & $U$ & 50 UGL \\
ABP 4 & ALTOT & $1998 Q 3$ & $J$ & 13.1 UGL
\end{tabular}


PBTOT Results for ABP 4

Data Retrieved by AWSA v1 from GIMS on 04MAR99 at 14:13

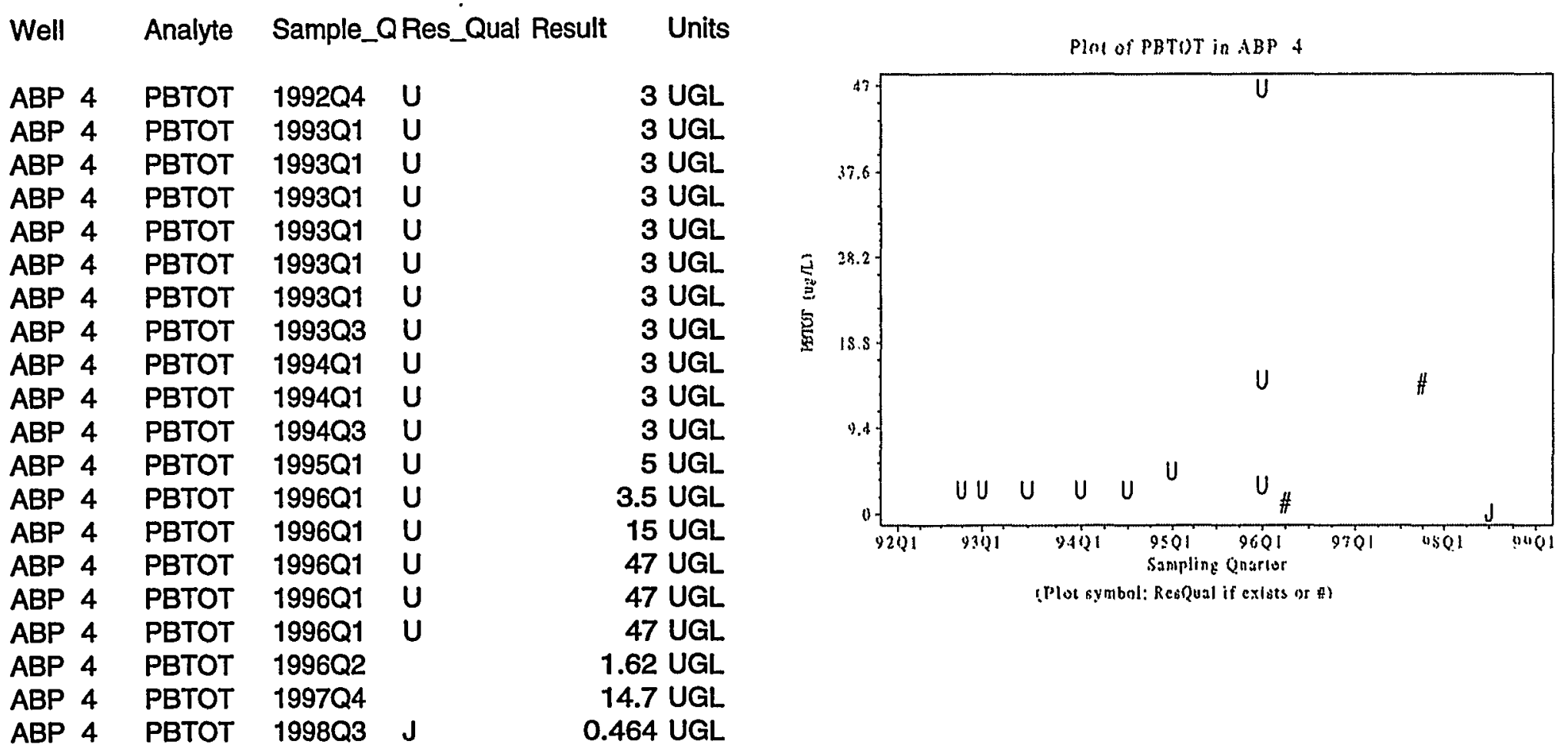



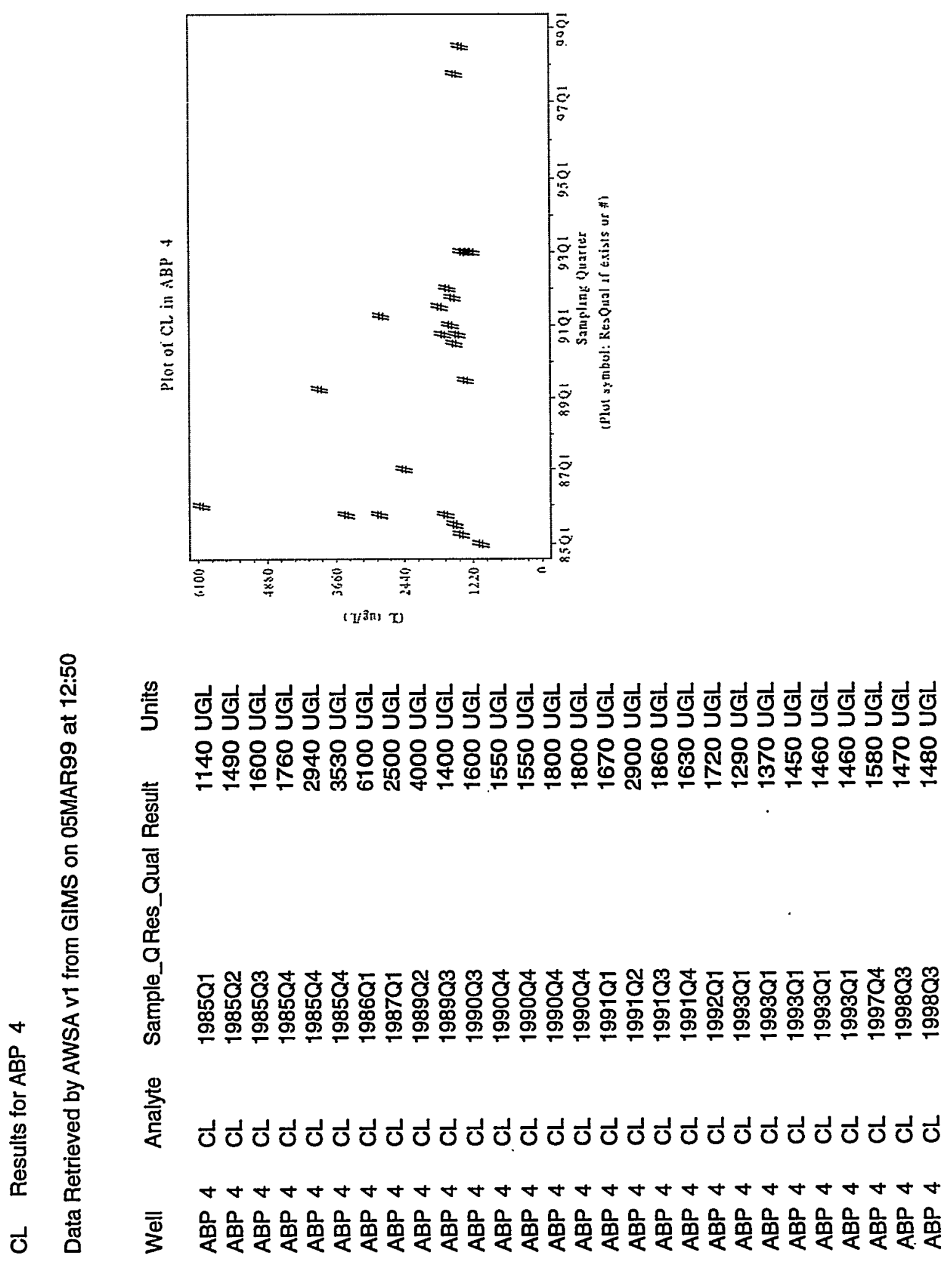
CCL4 Results for ABP 4

Data Retrieved by AWSA v1 from GIMS on 04MAR99 at 14:13

Well Analyte Sample_QRes_Qual Result Units

\begin{tabular}{|c|c|c|c|c|c|c|c|c|c|c|c|c|c|c|c|}
\hline ABP & 4 & CCL4 & 1985Q4 & & $5 \mathrm{UGL}$ & & $5 \cdot$ & $\#$ & $\#$ & $\mathrm{U}$ & $\bar{U}$ & UU & & & \\
\hline$A B P$ & 4 & CCL4 & 1985Q4 & & 5 UGL & & & & & & & & & & \\
\hline$A B P$ & 4 & CCL4 & 1986Q3 & & 2.49 UGL & & 41 & & & & & & & & \\
\hline$A B P$ & 4 & CCL4 & 1986Q4 & & $1 U G L$ & & $\left.{ }^{4}\right]$ & & & & & & & & \\
\hline$A B P$ & 4 & CCL4 & 1986Q4 & & $1 \mathrm{UGL}$ & & & & & & & & \# & & \\
\hline$A B P$ & 4 & CCL4 & 1987Q1 & & 1 UGL & 3 & 3. & & & 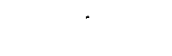 & & & & & \\
\hline$A B P$ & 4 & $\mathrm{CCL} 4$ & 1987Q1 & & 5 UGL & 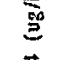 & & & $\#$ & & & $\#$ & & & \\
\hline ABP & 4 & CCL4 & 1987Q2 & & 1 UGL & $\vec{g}$ & 24 & & & & & & $\#$ & J & \\
\hline$A B P$ & 4 & CCL4 & 1987Q2 & & 1 UGL & & $\left.{ }^{2}\right]$ & & & & & & & J & \\
\hline$A B P$ & 4 & CCL4 & 1987Q3 & & $1 \mathrm{UGL}$ & & & & & & & & & y & \# \\
\hline$A B P$ & 4 & CCL4 & 1987Q4 & & 1 UGL & & 1. & & \#\#\#\# & H\# \# U U & U WU & JUNUUN & U & & \\
\hline$A B P$ & 4 & CCL4 & 1988Q1 & & $1 \mathrm{UGL}$ & & & & & & & & & & $\mathrm{J}$ \\
\hline$A B P$ & 4 & CCL4 & 1988Q1 & & 1 UGL & & & & & & & & & & \\
\hline ABP & 4 & CCL4 & 1988Q3 & & 1 UGL & & & 21 & 8721 & so? 21 & 9101 & 2301 & 9501 & $97 Q 1$ & 9901 \\
\hline ABP & 4 & CCL4 & 1989Q1 & $U$ & 1 UGL & & & & & & Snimpli & Qunriter & & & \\
\hline$A B P$ & 4 & CCL4 & $1989 Q 3$ & $U$ & 1 UGL & & & & & (Plo symplow & I: ResQu & 1 if exista ar & & & \\
\hline ABP & 4 & CCL4 & 1989Q3 & $U$ & 1 UGL & & & & & & & & & & \\
\hline ABP & 4 & CCL4 & 1989Q3 & U & 5 UGL & & & & & & & & & & \\
\hline$A B P$ & 4 & CCL4 & 1989Q3 & $U$ & 5 UGL & & & & & & & & & & \\
\hline ABP & 4 & CCL4 & 1990Q1 & & 0.73 UGL & & & & & & & & & & \\
\hline ABP & 4 & CCL4 & 1990Q1 & & $1.04 \mathrm{UGL}$ & & & & & & & & & & \\
\hline ABP & 4 & CCL4 & 1990Q1 & $\mathbf{U}$ & 1 UGL & & & & & & & & & & \\
\hline ABP & 4 & CCL4 & 1990Q1 & $U$ & 1 UGL & & & & & & & & & & \\
\hline ABP & 4 & CCL4 & 1990Q3 & U & 1 UGL & & & & & & & & & & \\
\hline$A B P$ & 4 & CCL4 & 1990Q4 & U & 5 UGL & & & & & & & & & & \\
\hline ABP & 4 & CCL4 & 1990Q4 & U & 5 UGL & & & & & & & & & & \\
\hline ABP & 4 & CCL4 & 1990Q4 & $\mathrm{U}$ & 5 UGL & & & & & & & & & & \\
\hline ABP & 4 & CCL4 & 1990Q4 & U & 5 UGL & & & & & & & & & & \\
\hline$A B F$ & 4 & CCL4 & 1991Q1 & U & $1 \mathrm{UGL}$ & & & & & & & & & & \\
\hline $\mathrm{AE}$ & 4 & CCL4 & 1991Q2 & $\mathbf{U}$ & 1 UGL & & & & & & & & & & \\
\hline
\end{tabular}




\begin{tabular}{|c|c|c|c|c|c|}
\hline ABP & 4 & CCL4 & 1991Q3 & $U$ & $1 \mathrm{UGL}$ \\
\hline ABP & 4 & CCL4 & 1991Q4 & U & $1 \mathrm{UGL}$ \\
\hline ABP & 4 & CCL4 & 1992Q1 & $U$ & $1 \mathrm{UGL}$ \\
\hline ABP & 4 & CCL4 & 1992Q2 & U & $1 U G$ \\
\hline ABP & 4 & CCL4 & 1992Q3 & U & $1 U G$ \\
\hline ABP & 4 & CCL4 & 1992Q3 & U & $1 \mathrm{UG}$ \\
\hline ABP & 4 & CCL4 & 1992Q3 & $U$ & $1 \mathrm{UGl}$ \\
\hline ABP & 4 & CCL4 & 1992Q3 & $U$ & 5 UGI \\
\hline ABP & 4 & CCL4 & 1992Q3 & $U$ & 5 UGI \\
\hline ABP & 4 & CCL4 & 1992Q4 & $U$ & $1 \mathrm{UGI}$ \\
\hline ABP & 4 & CCL4 & 1993Q1 & U & $1 \mathrm{UG}$ \\
\hline ABP & 4 & CCL4 & 1993Q1 & $U$ & $1 U G$ \\
\hline ABP & 4 & CCL4 & 1993Q1 & $U$ & 5 UG \\
\hline ABP & 4 & CCL4 & 1993Q1 & $U$ & 5 UG \\
\hline ABP & 4 & CCL4 & 1993Q2 & $U$ & $1 \mathrm{UG}$ \\
\hline ABP & 4 & CCL4 & 1993Q3 & UJ & $1 \mathrm{UG}$ \\
\hline ABP & 4 & CCL4 & 1993Q4 & $U$ & $1 \mathrm{UG}$ \\
\hline ABP & 4 & CCL4 & 1993Q4 & $U$ & 1 UGL \\
\hline ABP & 4 & CCL4 & 1994Q1 & & $2.4 \mathrm{UGL}$ \\
\hline ABP & 4 & CCL4 & 1994Q1 & & $2.4 \mathrm{UGL}$ \\
\hline ABP & 4 & CCL4 & 1994Q2 & & 3.3 UGL \\
\hline ABP & 4 & CCL4 & 1994Q3 & $u$ & $1 \mathrm{UGL}$ \\
\hline ABP & 4 & CCL4 & 1995Q1 & & $2.19 \mathrm{UGL}$ \\
\hline ABP & 4 & CCL4 & 1996Q1 & $J$ & $1.2 \mathrm{UGL}$ \\
\hline ABP & 4 & CCL4 & 1996Q1 & $J$ & $1.35 \mathrm{UGL}$ \\
\hline ABP & 4 & CCL4 & 1996Q1 & $\mathrm{J}$ & $1.48 \mathrm{UGL}$ \\
\hline ABP & 4 & CCL4 & 1996Q1 & $J$ & $1.88 \mathrm{UGL}$ \\
\hline ABP & 4 & CCL4 & 1996Q1 & $J$ & 2.17 UGL \\
\hline ABP & 4 & CCL4 & 1996Q2 & $J$ & 1.5 \\
\hline ABP & 4 & CCL4 & 1997Q4 & & 1.49 \\
\hline ABP & 4 & CCL4 & 1998Q3 & $J$ & 0.511 \\
\hline
\end{tabular}


TCLEE Results for ABP 4

Data Retrieved by AWSA v1 from GIMS on 04MAR99 at 14:13

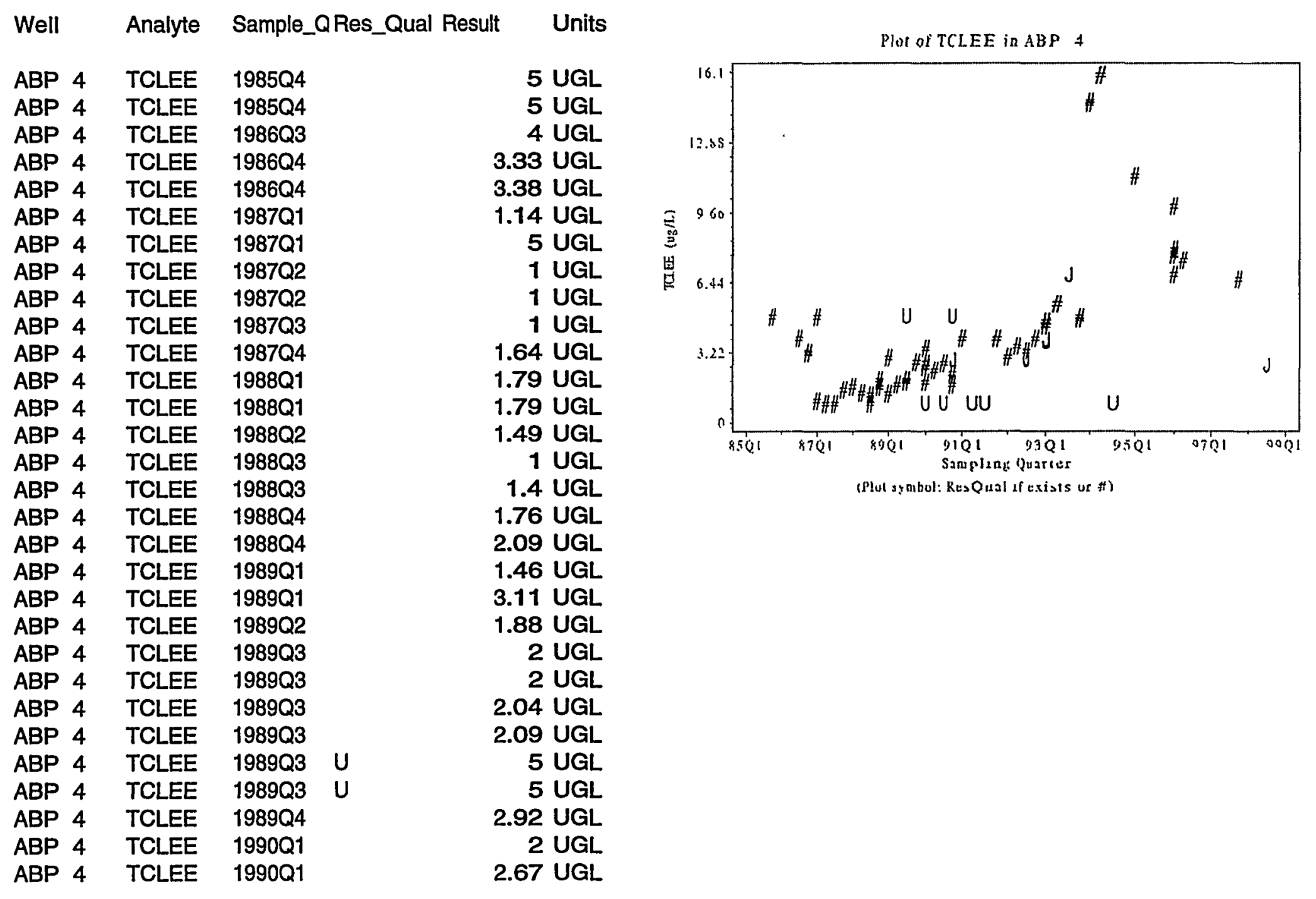




\begin{tabular}{|c|c|c|c|c|}
\hline ABP & TCLEE & 1990Q1 & & $2.71 \mathrm{UGL}$ \\
\hline ABP & TCLEE & 1990Q1 & & $2.87 \mathrm{UGL}$ \\
\hline ABP & TCLEE & 1990Q1 & & $3.54 \mathrm{UGL}$ \\
\hline ABP & TCLEE & 1990Q1 & $\mathbf{U}$ & 1 UGL \\
\hline ABP & TCLEE & 1990Q2 & & 2.53 UGL \\
\hline ABP & TCLEE & $1990 Q 3$ & & 2.86 UGL \\
\hline ABP & TCLEE & 1990Q3 & $\mathbf{U}$ & 1 UGL \\
\hline ABP & TCLEE & 1990Q4 & & 1.87 UGL \\
\hline ABP & TCLEE & 1990Q4 & & 2.39 UGL \\
\hline ABP & TCLEE & 1990Q4 & $J$ & 3 UGL \\
\hline ABP & TCLEE & 1990Q4 & $\mathbf{J}$ & 3 UGL \\
\hline ABP & TCLEE & 1990Q4 & $\mathbf{U}$ & 5 UGL \\
\hline ABP & TCLEE & 1990Q4 & $\mathbf{U}$ & 5 UGL \\
\hline ABP & TCLEE & 1991Q1 & & 4 UGL \\
\hline ABP & TCLEE & 1991Q2 & $\mathbf{U}$ & 1 UGL \\
\hline ABP & TCLEE & 1991Q3 & $U$ & 1 UGL \\
\hline ABP & TCLEE & 1991Q4 & & 4 UGL \\
\hline ABP & TCLEE & 1992Q1 & & 3.15 UGL \\
\hline ABP & TCLEE & 1992Q2 & & 3.64 UGL \\
\hline ABP & TCLEE & 1992Q3 & & 3.42 UGL \\
\hline ABP & TCLEE & 1992Q3 & & 3.43 UGL \\
\hline$A B P$ & TCLEE & 1992Q3 & & 3.43 UGL \\
\hline ABP & TCLEE & 1992Q3 & $J$ & 3 UGL \\
\hline ABP & TCLEE & 1992Q3 & $\mathbf{J}$ & 3.08 UGL \\
\hline ABP & TCLEE & 1992Q4 & & 4 UGL \\
\hline ABP & TCLEE & 1993Q1 & & 4.6 UGL \\
\hline ABP & TCLEE & 1993Q1 & & 4.8 UGL \\
\hline ABP & TCLEE & 1993Q1 & J & 3.84 UGL \\
\hline ABP & TCLEE & 1993Q1 & $J$ & 3.95 UGL \\
\hline ABP & TCLEE & 1993Q2 & & 5.6 UGL \\
\hline ABP & TCLEE & 1993Q3 & $\mathbf{J}$ & 6.91 UGL \\
\hline ABP & TCLEE & 1993Q4 & & 4.9 UGL \\
\hline ABP & TCLEE & 1993Q4 & & 5 UGL \\
\hline ABP & TCLEE & 1994Q1 & & 14.8 UGL \\
\hline ABP & TCLEE & 1994Q1 & & 14.9 UGL \\
\hline ABP & TCLEE & 1994Q2 & & 16.1 UGL \\
\hline ABP & TCLEE & 1994Q3 & $U$ & 1 UGL \\
\hline
\end{tabular}


ABP 4 TCLEE 1995Q1

ABP 4 TCLEE 1996Q1

ABP 4 TCLEE 1996Q1

ABP 4 TCLEE 1996Q1

ABP 4 TCLEE 1996Q1

ABP 4 TCLEE 1996Q1

ABP 4 TCLEE 1996Q2

ABP 4 TCLEE 1997Q4

ABP 4 TCLEE 1998Q3 J
$11.5 \mathrm{UGL}$

6.96 UGL

7.85 UGL

7.87 UGL

8.1 UGL

10.1 UGL

7.61 UGL

6.74 UGL

2.76 UGL 


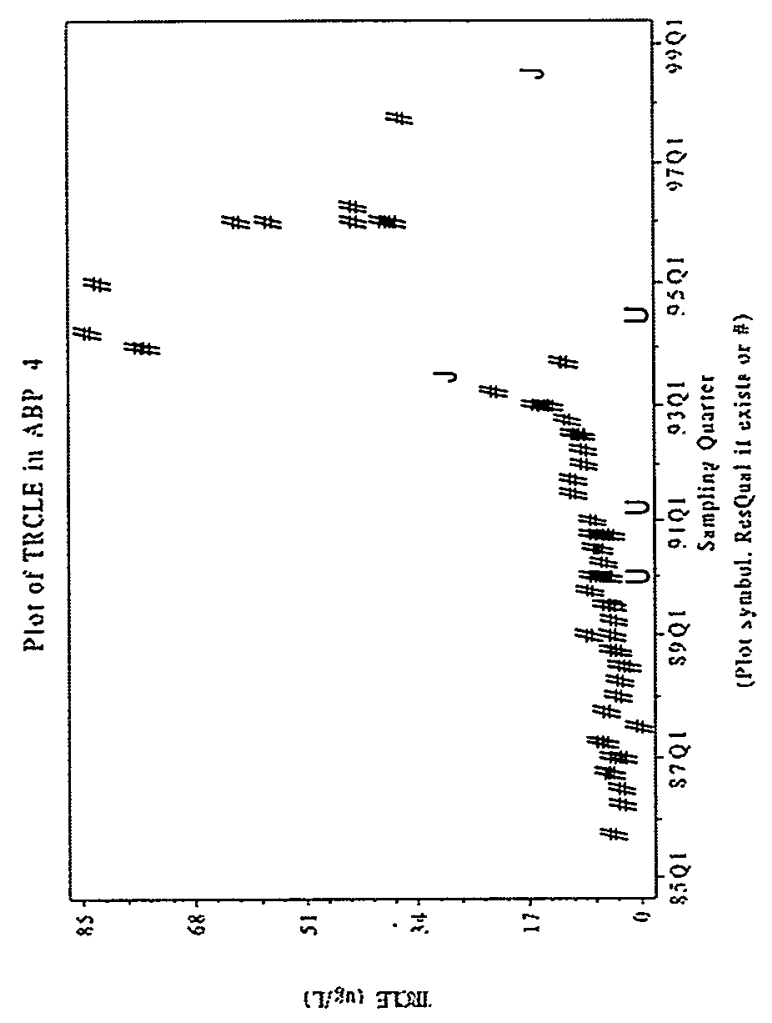

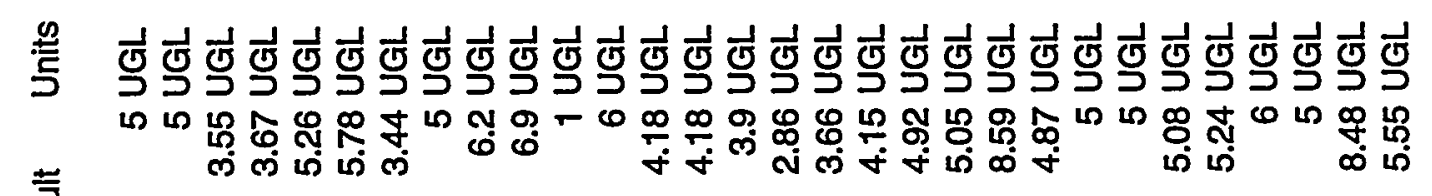

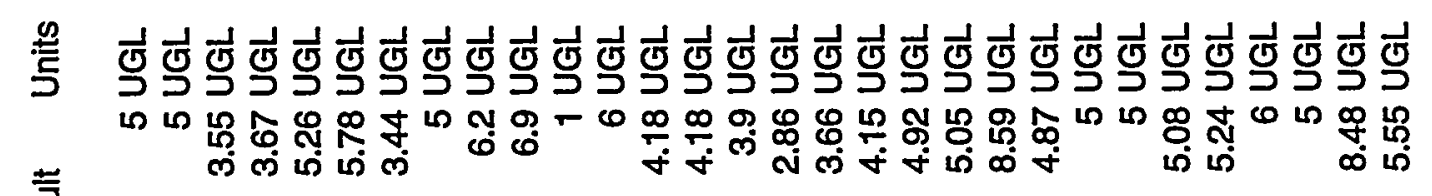

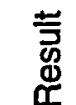
$\underline{\pi}$ $\overline{\widetilde{g}}$ $\stackrel{\mathscr{g}}{\mathbb{1}}$

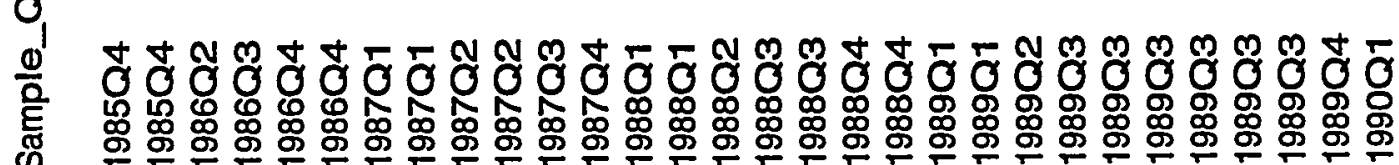

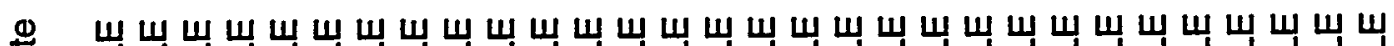
خ

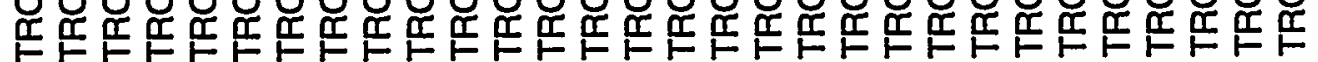

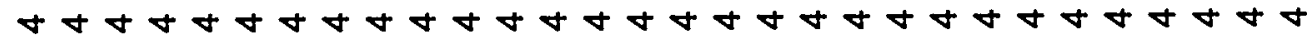

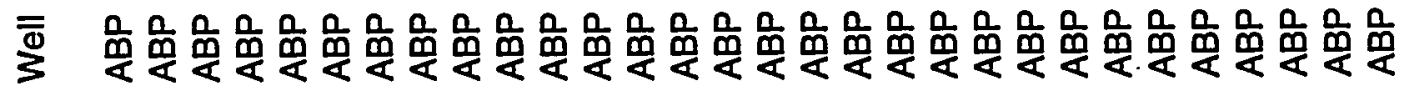




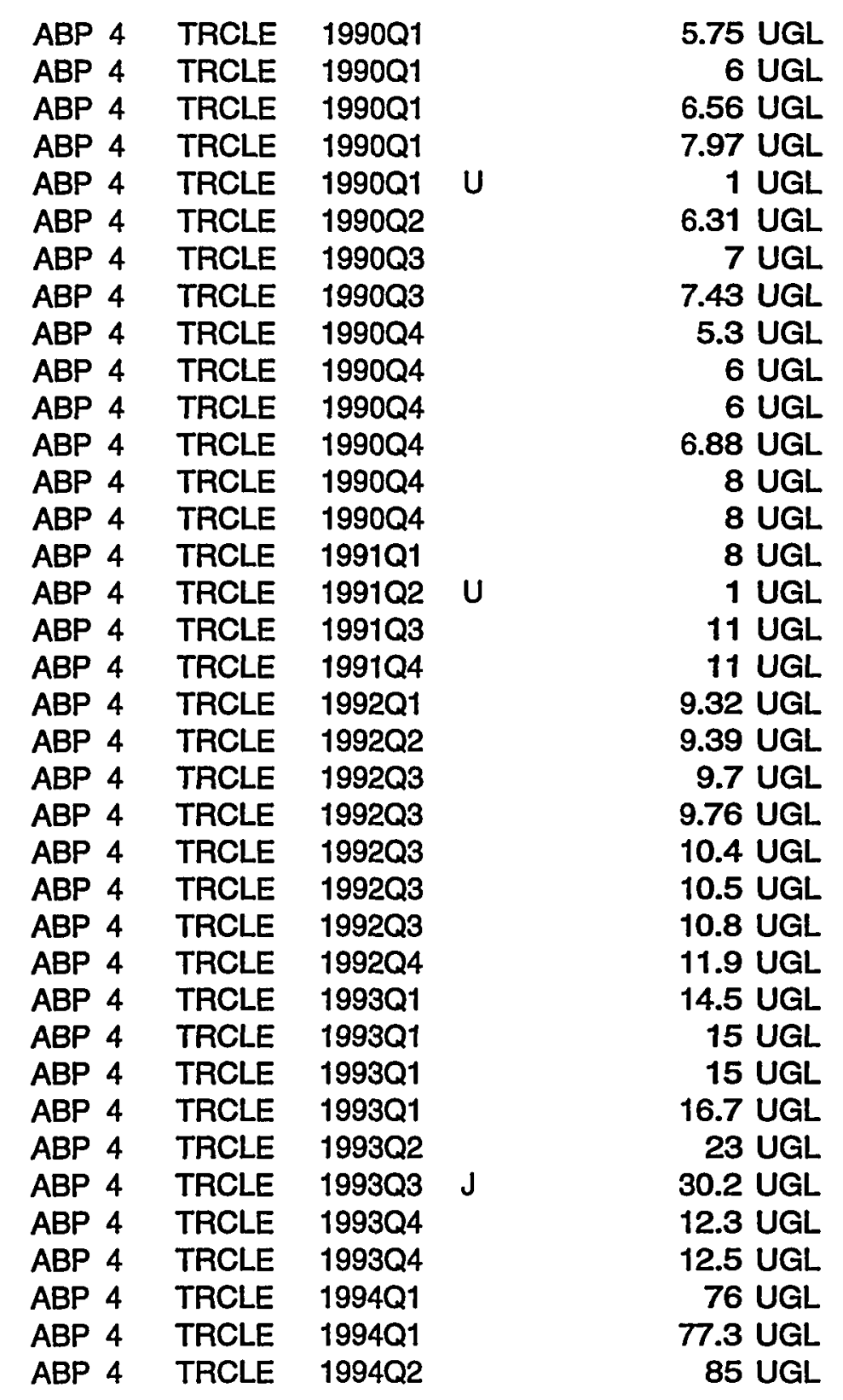




\begin{tabular}{|c|c|c|c|c|}
\hline ABP 4 & TRCLE & 1994Q3 & $u$ & 1 UGL \\
\hline ABP 4 & TRCLE & 1995Q1 & & 83.4 UGL \\
\hline ABP 4 & TRCLE & 1996Q1 & & 38.4 UGL \\
\hline ABP 4 & TRCLE & 1996Q1 & & 39.9 UGL \\
\hline ABP 4 & TRCLE & 1996Q1 & & 44.4 UGL \\
\hline ABP 4 & TRCLE & 1996Q1 & & 57.4 UGL \\
\hline ABP 4 & TRCLE & 1996Q1 & & 62.3 UGL \\
\hline ABP 4 & TRCLE & 1996Q2 & & 44.4 UGL \\
\hline $\mathrm{ABP} 4$ & TRCLE & 1997Q4 & & 37.2 UGL \\
\hline$A B P$ & TRCLE & $1998 \mathrm{Q} 3$ & $J$ & 16.7 UGL \\
\hline
\end{tabular}


ALPHAG Results for ABP 4

Data Retrieved by AWSA v1 from GIMS on 04MAR99 at 14:13

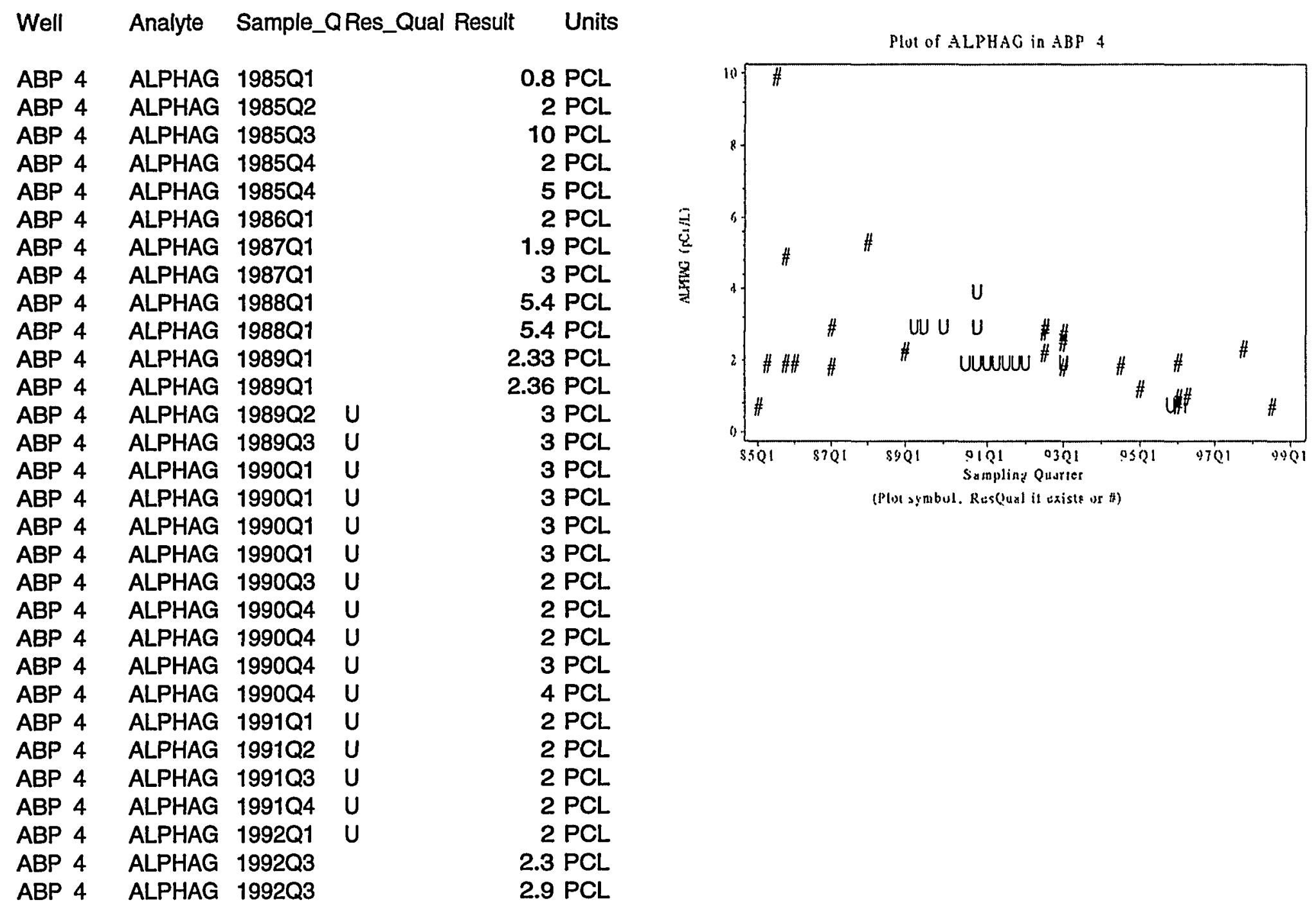




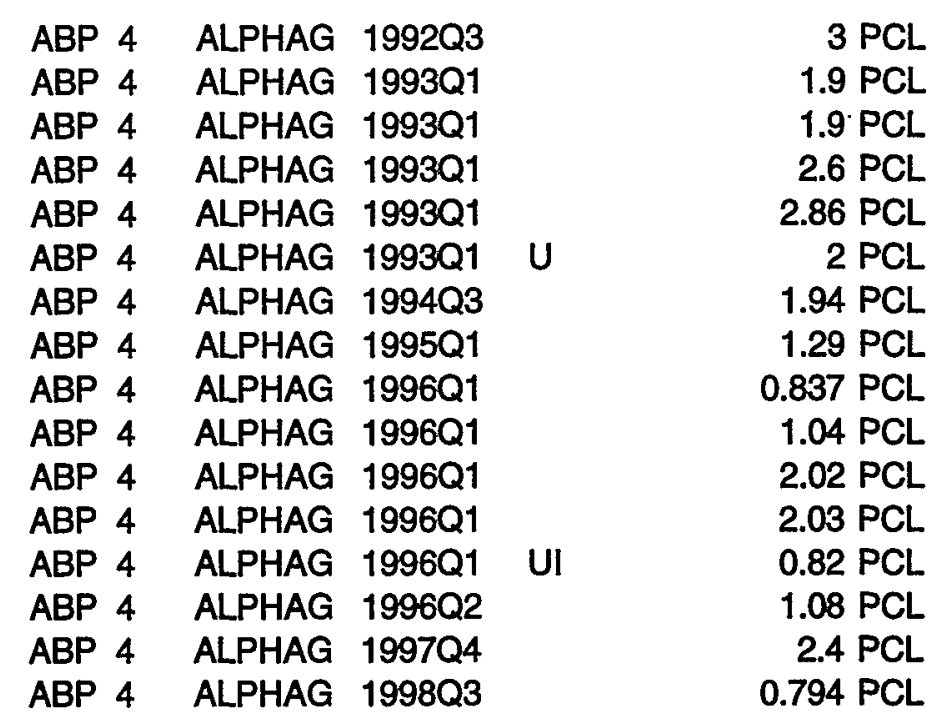




\section{WELL ARP-3 DATA}


FPH Results for ARP 3

Data Retrieved by AWSA v1 from GIMS on 05MAR99 at 13:20

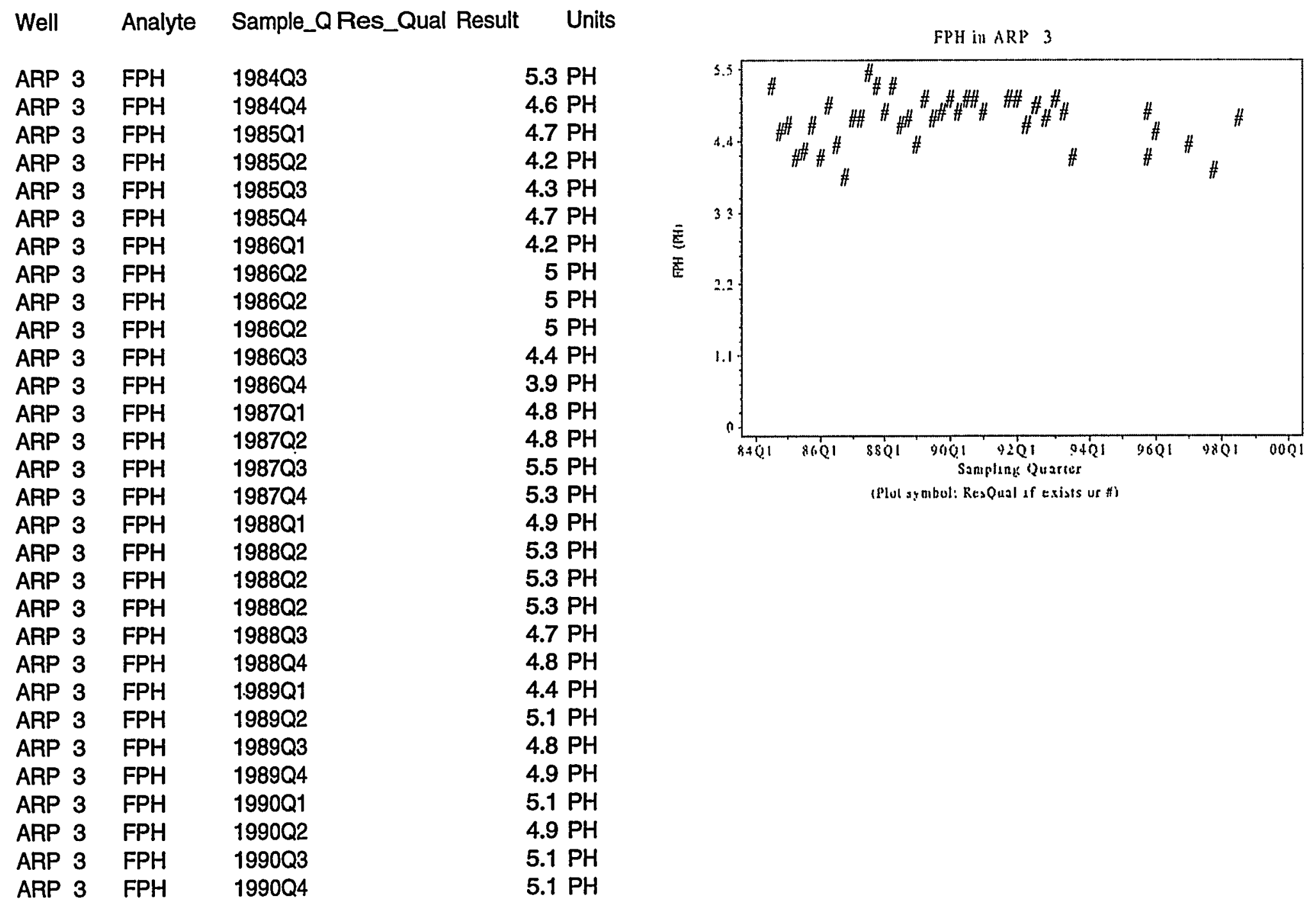




\begin{tabular}{|c|c|c|c|}
\hline ARP 3 & FPH & 1991Q1 & $4.9 \mathrm{PH}$ \\
\hline ARP 3 & FPH & 1991Q4 & $5.1 \mathrm{PH}$ \\
\hline ARP 3 & FPH & 1992Q1 & $5.1 \mathrm{PH}$ \\
\hline ARP 3 & FPH & 1992Q1 & $5.1 \mathrm{PH}$ \\
\hline ARP 3 & FPH & 1992Q2 & $4.7 \mathrm{PH}$ \\
\hline ARP 3 & FPH & 1992Q2 & $4.7 \mathrm{PH}$ \\
\hline ARP 3 & FPH & 1992Q3 & $5 \mathrm{PH}$ \\
\hline ARP 3 & FPH & $1992 Q 4$ & $4.8 \mathrm{PH}$ \\
\hline ARP 3 & FPH & 1993Q1 & $5.1 \mathrm{PH}$ \\
\hline ARP 3 & FPH & 1993Q2 & $4.9 \mathrm{PH}$ \\
\hline ARP 3 & FPH & 1993Q3 & 4.2 $\mathrm{PH}$ \\
\hline ARP 3 & FPH & $1995 Q 4$ & $4.2 \mathrm{PH}$ \\
\hline ARP 3 & FPH & 1995Q4 & $4.9 \mathrm{PH}$ \\
\hline ARP 3 & FPH & 1996Q1 & $4.6 \mathrm{PH}$ \\
\hline ARP 3 & FPH & 1997Q1 & $4.4 \mathrm{PH}$ \\
\hline ARP 3 & FPH & 1997Q4 & $4 \mathrm{PH}$ \\
\hline ARP 3 & FPH & 1998Q3 & $4.8 \mathrm{PH}$ \\
\hline
\end{tabular}


COND Results for ARP 3

Data Retrieved by AWSA v1 from GIMS on 05MAR99 at 13:20

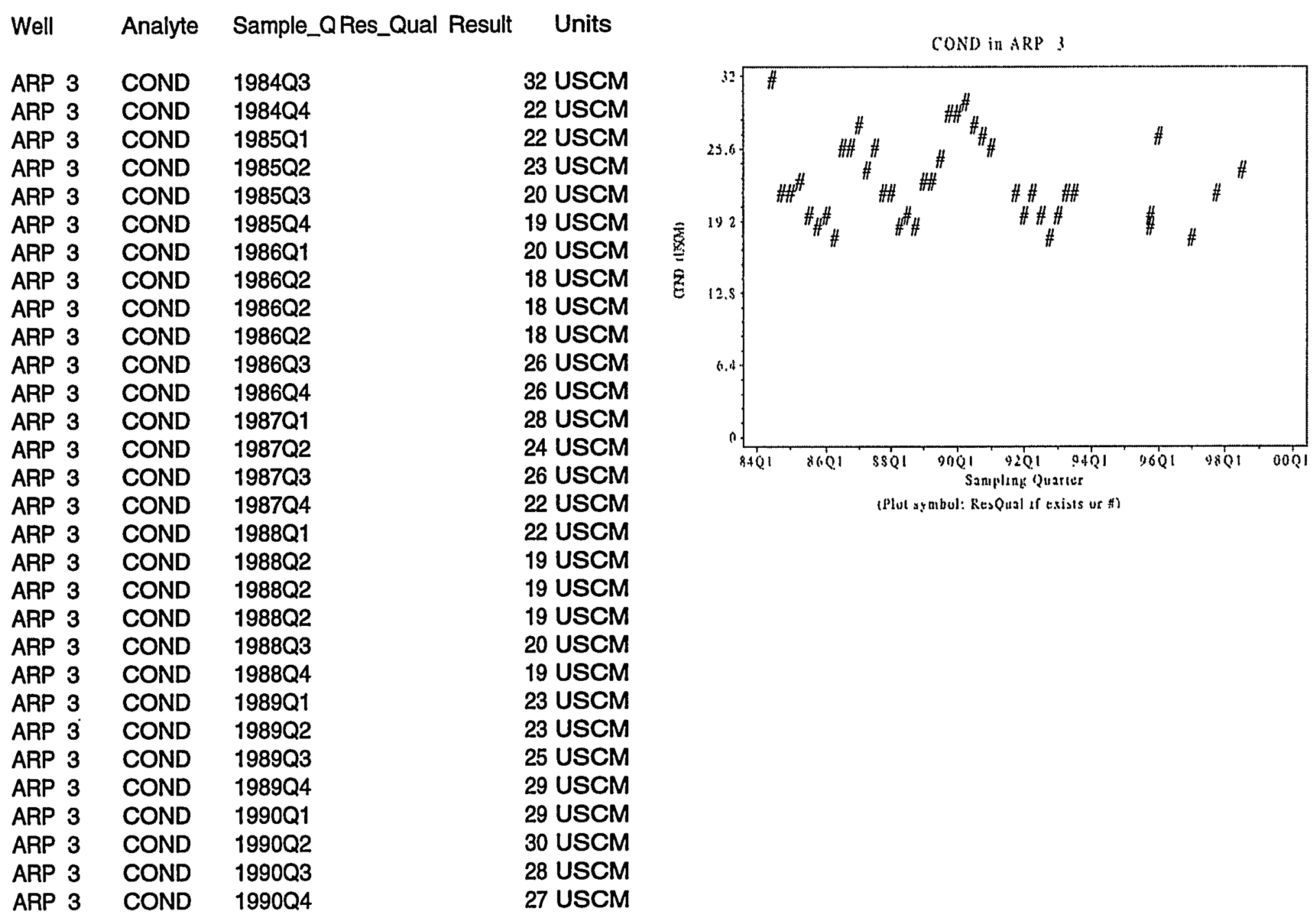




$\begin{array}{llll}\text { ARP 3 } & \text { COND } & 1991 Q 1 & 26 \text { USCM } \\ \text { ARP 3 } & \text { COND } & 1991 Q 4 & 22 \text { USCM } \\ \text { ARP 3 } & \text { COND } & 1992 Q 1 & 20 \text { USCM } \\ \text { ARP 3 } & \text { COND } & 1992 Q 1 & 20 \text { USCM } \\ \text { ARP 3 } & \text { COND } & 1992 Q 2 & 22 \text { USCM } \\ \text { ARP 3 } & \text { COND } & 1992 Q 2 & 22 \text { USCM } \\ \text { ARP 3 } & \text { COND } & 1992 Q 3 & 20 \text { USCM } \\ \text { ARP 3 } & \text { COND } & 1992 Q 4 & 18 \text { USCM } \\ \text { ARP 3 } & \text { COND } & 1993 Q 1 & 20 \text { USCM } \\ \text { ARP 3 } & \text { COND } & 1993 Q 2 & 22 \text { USCM } \\ \text { ARP 3 } & \text { COND } & 1993 Q 3 & 22 \text { USCM } \\ \text { ARP 3 } & \text { COND } & 1995 Q 4 & 19 \text { USCM } \\ \text { ARP 3 } & \text { COND } & 1995 Q 4 & 20 \text { USCM } \\ \text { ARP 3 } & \text { COND } & 1996 Q 1 & 27 \text { USCM } \\ \text { ARP 3 } & \text { COND } & 1997 Q 1 & 18 \text { USCM } \\ \text { ARP 3 } & \text { COND } & 1997 Q 4 & 22 \text { USCM } \\ \text { ARP 3 } & \text { COND } & 1998 Q 3 & 24 \text { USCM }\end{array}$


CATOT Results for ARP 3

Data Retrieved by AWSA v1 from GIMS on 05MAR99 at 12:50

Well Analyte Sample_QRes_Qual Result Units ARP $3 \quad$ CATOT 1993Q1 355 UGL

ARP 3 CATOT 1995Q4 441 UGL

ARP 3 CATOT 1995Q4 483 UGL

ARP 3 CATOT 1997Q4 373 UGL

ARP 3 CATOT 1998Q3 521 UGL

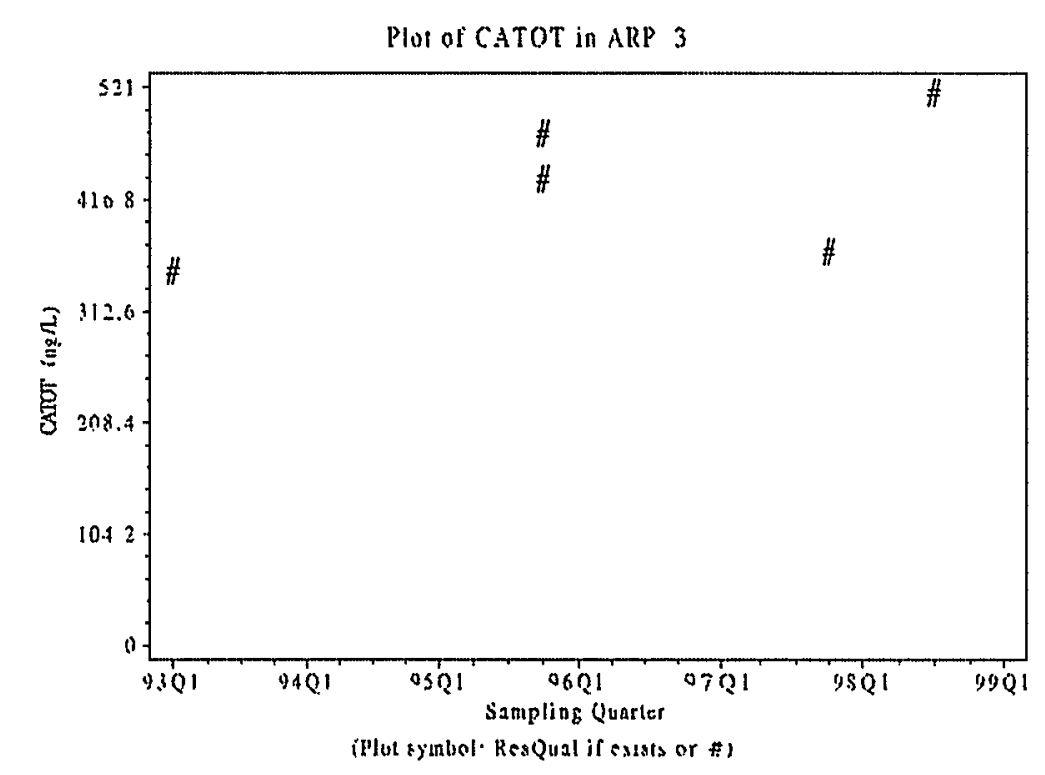


KTOT Results for ARP 3

Data Retrieved by AWSA v1 from GIMS on 05MAR99 at 12:50

\section{Well Analyte Sample_QRes_Qual Result Units}

ARP 3 KTOT 1993Q1 U 500 UGL

ARP 3 KTOT $1995 \mathrm{Q} 4 \mathrm{~J} \quad 149 \mathrm{UGL}$

ARP 3 KTOT 1995Q4 J $206 \mathrm{UGL}$

ARP 3 KTOT 1997Q4 174 UGL

$\begin{array}{lll}\text { ARP } 3 & \text { KTOT } 1998 Q 3 & 217 \text { UGL }\end{array}$

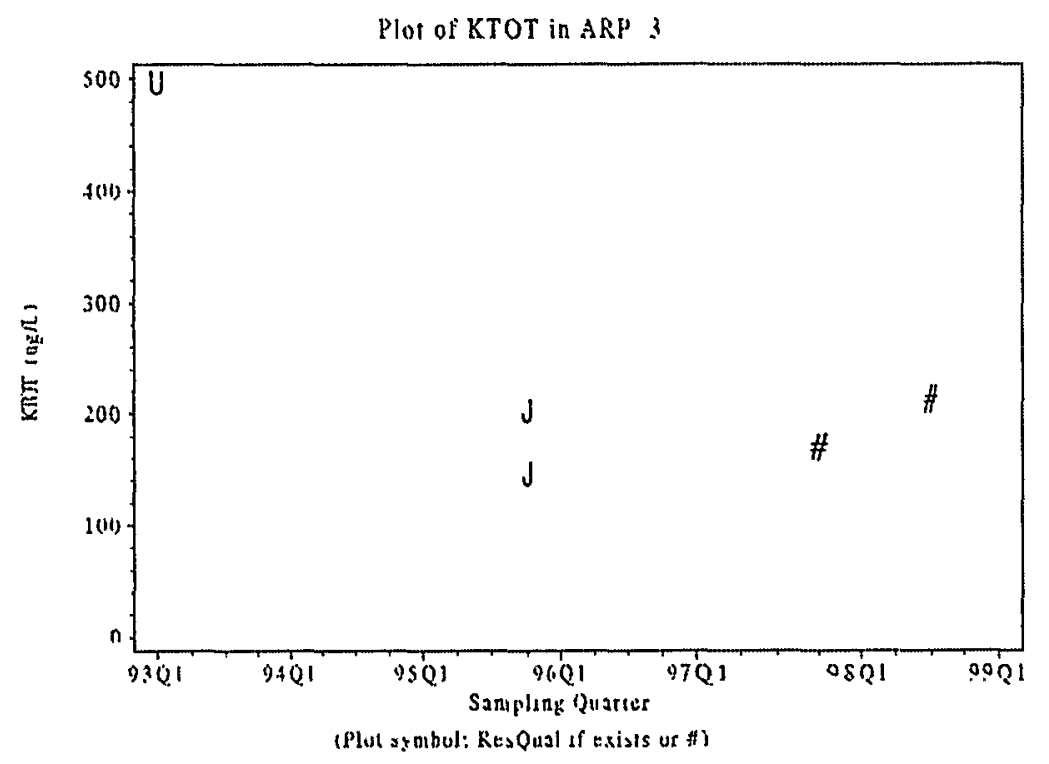


NATOT Results for ARP 3

Data Retrieved by AWSA v1 from GIMS on 05MAR99 at 12:50

$\begin{array}{llll}\text { Well } & \text { Analyte } & \text { Sample_QRes_Qual Result } & \text { Units } \\ & & & \\ \text { ARP 3 } & \text { NATOT } & 1993 Q 1 & 1970 \text { UGL } \\ \text { ARP 3 } & \text { NATOT } & \text { 1995Q4 } & 1770 \text { UGL } \\ \text { ARP 3 } & \text { NATOT } & \text { 1995Q4 } & 1790 \text { UGL } \\ \text { ARP 3 } & \text { NATOT } & \text { 1997Q4 } & \text { 2030 UGL } \\ \text { ARP 3 } & \text { NATOT } & 1998 Q 3 & 2570 \text { UGL }\end{array}$

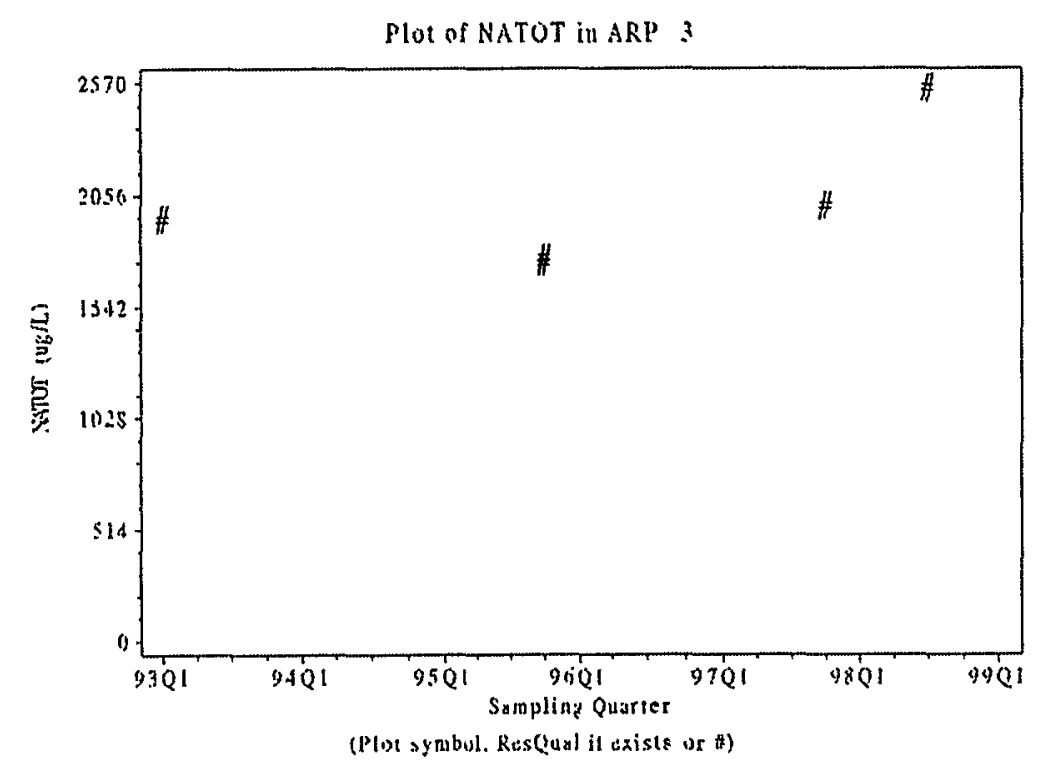



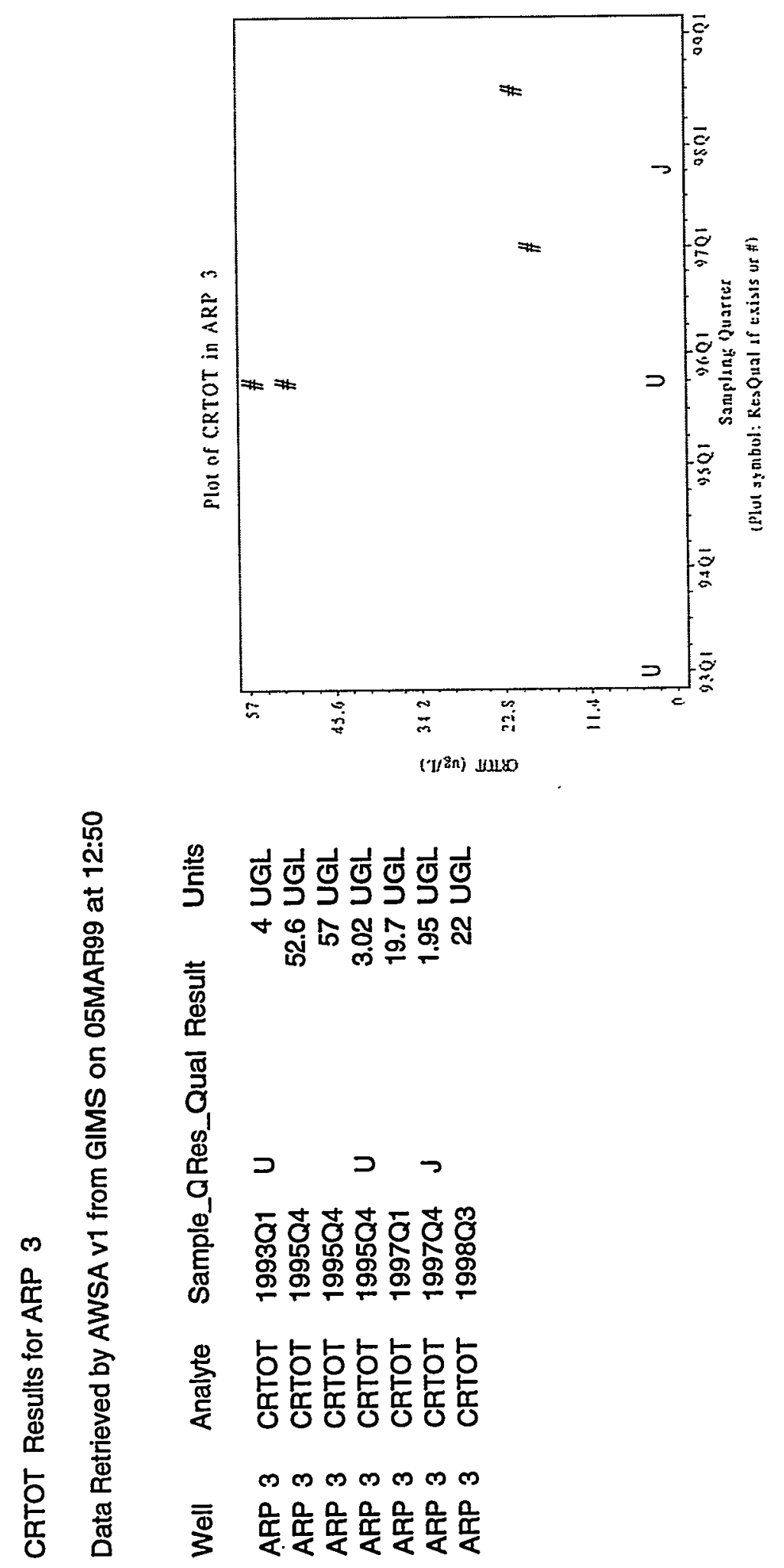


\section{FETOT Results for ARP 3}

Data Retrieved by AWSA v1 from GIMS on 05MAR99 at 12:50

Well Analyte Sample_QRes_Qual Result Units

ARP 3 FETOT 1993Q1

ARP 3 FETOT 1995Q4

ARP 3 FETOT 1995Q4

ARP 3 FETOT 1995Q4

ARP 3 FETOT 1997Q1

ARP 3 FETOT $1998 Q 3$

65.2 UGL

84.7 UGL

$7440 \mathrm{UGL}$

8400 UGL

586 UGL

5850 UGL

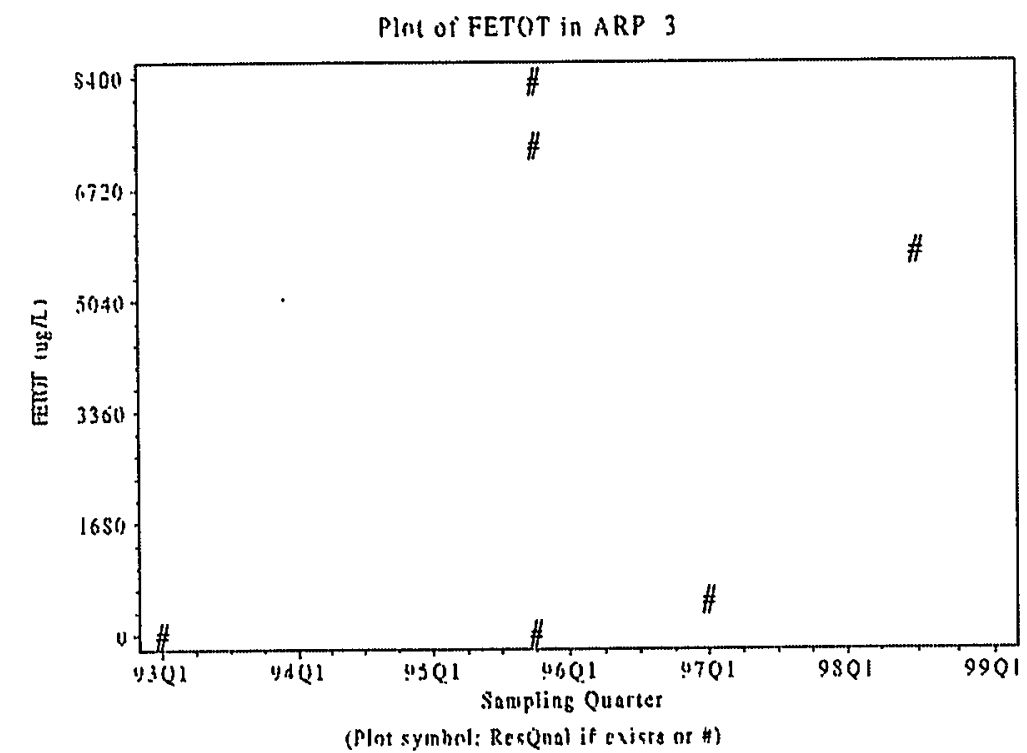


MNTOT Results for ARP 3

Data Retrieved by AWSA V1 from GIMS on 05MAR99 at 12:50

\begin{tabular}{|c|c|c|c|c|}
\hline Well & Analyte & Sample_C & Res_Qual Result & Units \\
\hline ARP 3 & MNTOT & 1993Q1 & & 7.36 UGL \\
\hline ARP 3 & MNTOT & 1995Q4 & & 5.32 UGL \\
\hline ARP 3 & MNTOT & 1995Q4 & & $40.5 \mathrm{UGL}$ \\
\hline ARP 3 & MNTOT & 1996Q1 & & 24.3 UGL \\
\hline ARP 3 & MNTOT & 1997Q1 & & 8.4 UGL \\
\hline ARP 3 & MNTOT & 1997Q4 & 了 & $6.59 \mathrm{UGL}$ \\
\hline ARP 3 & MNTOT & $1998 Q 3$ & & 44.2 UGL \\
\hline
\end{tabular}

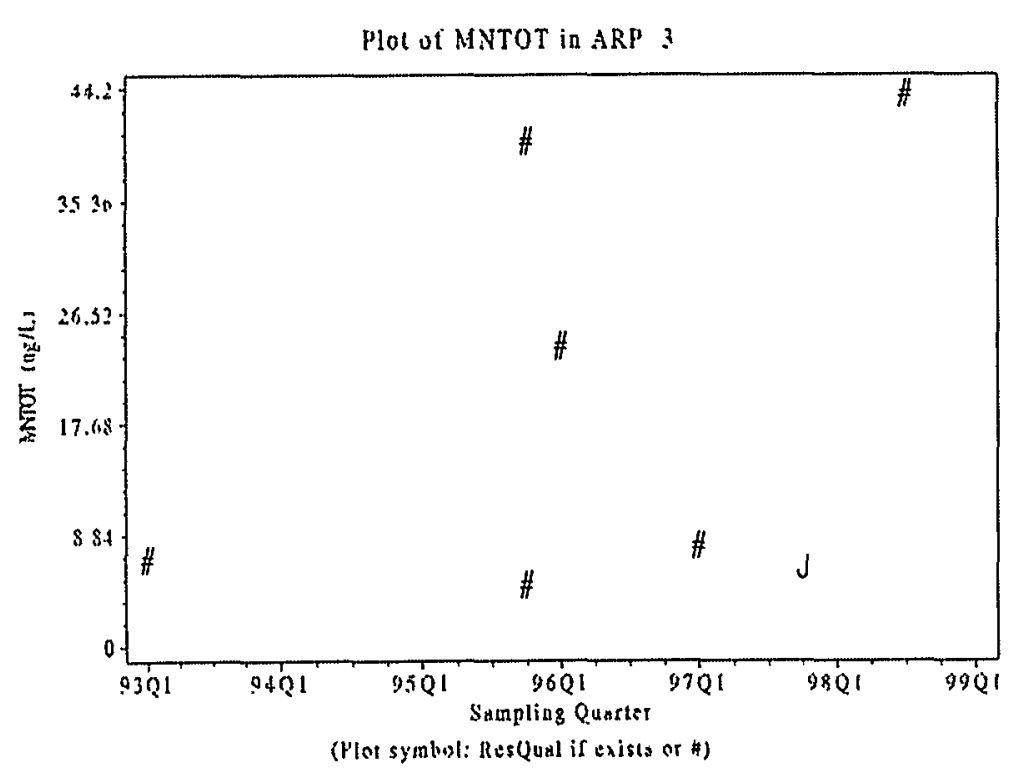


ALTOT Results for ARP 3

Data Retrieved by AWSA v1 from GIMS on 05MAR99 at 12:50

Well Analyte Sample_QRes_Qual Result Units

ARP 3 ALTOT 199301

ARP 3 ALTOT $1993 \mathrm{Q} 3$ UJ $20 \mathrm{UGL}$

ARP 3 ALTOT 1995Q4 31.2 UGL

ARP 3 ALTOT 1995Q4 1540 UGL

ARP 3 ALTOT 1995Q4 2950 UGL

ARP 3 ALTOT 1996Q1 J 1380 UGL

ARP 3 ALTOT 1996Q1 J 1530 UGL

ARP 3 ALTOT 1997Q1 $106 \mathrm{UGL}$

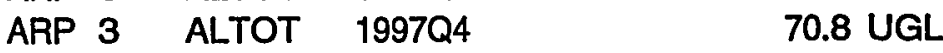

ARP 3 ALTOT 1998Q3 1290 UGL

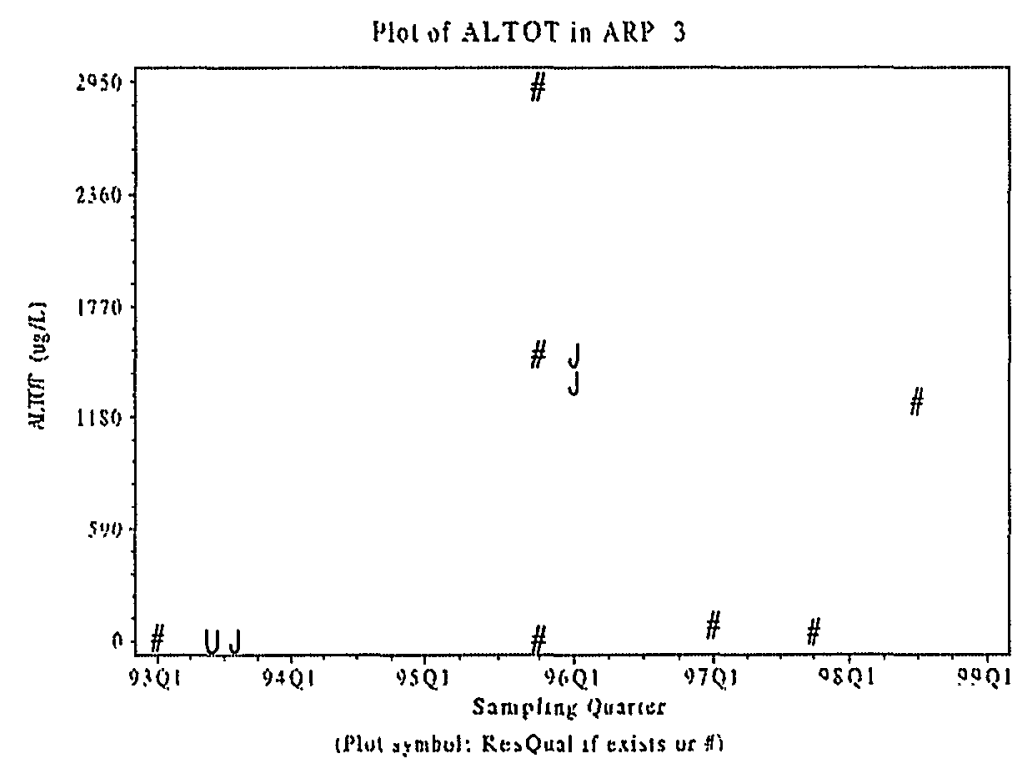



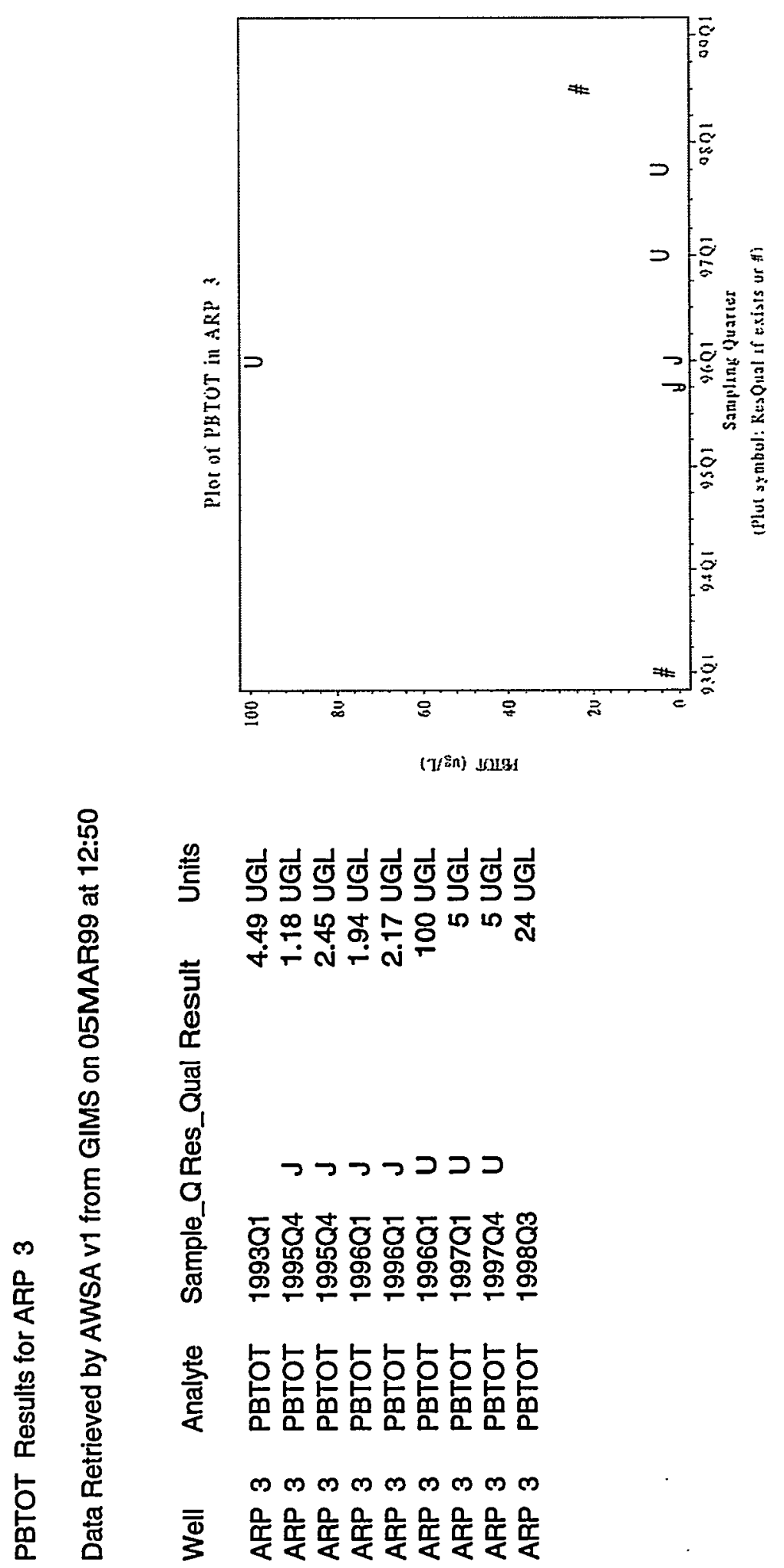
CL Results for ARP 3

Data Retrieved by AWSA v1 from GIMS on 05MAR99 at 12:50

Well Analyte Sample_QRes_Qual Result Units

\begin{tabular}{|c|c|c|c|}
\hline ARP & $\mathrm{CL}$ & 1984Q3 & $3000 \mathrm{UGL}$ \\
\hline ARP & $\mathrm{CL}$ & $1984 Q 4$ & $3100 \mathrm{UGL}$ \\
\hline ARP 3 & $\mathrm{CL}$ & 1984Q4 & 3500 UGL \\
\hline ARP 3 & $\mathrm{CL}$ & 1985Q1 & 2000 UGL \\
\hline ARP 3 & CL & 1985Q2 & 2730 UGL \\
\hline ARP 3 & CL & 1986Q1 & 6900 UGL \\
\hline ARP 3 & CL & 1986Q1 & 6900 UGL \\
\hline ARP 3 & CL & 1987Q1 & 3100 UGL \\
\hline ARP 3 & $\mathrm{CL}$ & 1989Q1 & 2900 UGL \\
\hline ARP 3 & $\mathrm{CL}$ & 1989Q2 & 7000 UGL \\
\hline ARP 3 & $\mathrm{CL}$ & 1991Q1 & 3250 UGL \\
\hline ARP 3 & $\mathrm{CL}$ & 1993Q1 & 2540 UGL \\
\hline ARP 3 & $\mathrm{CL}$ & 1997Q1 & 2430 UGL \\
\hline ARP 3 & $\mathrm{CL}$ & 1997Q1 & 2470 UGL \\
\hline ARP & $\mathrm{CL}$ & 1997Q4 & 2570 UGL \\
\hline ARP & $\mathrm{CL}$ & 1997Q4 & $2580 \cup G$ \\
\hline & C & 1998Q3 & 2640 \\
\hline
\end{tabular}

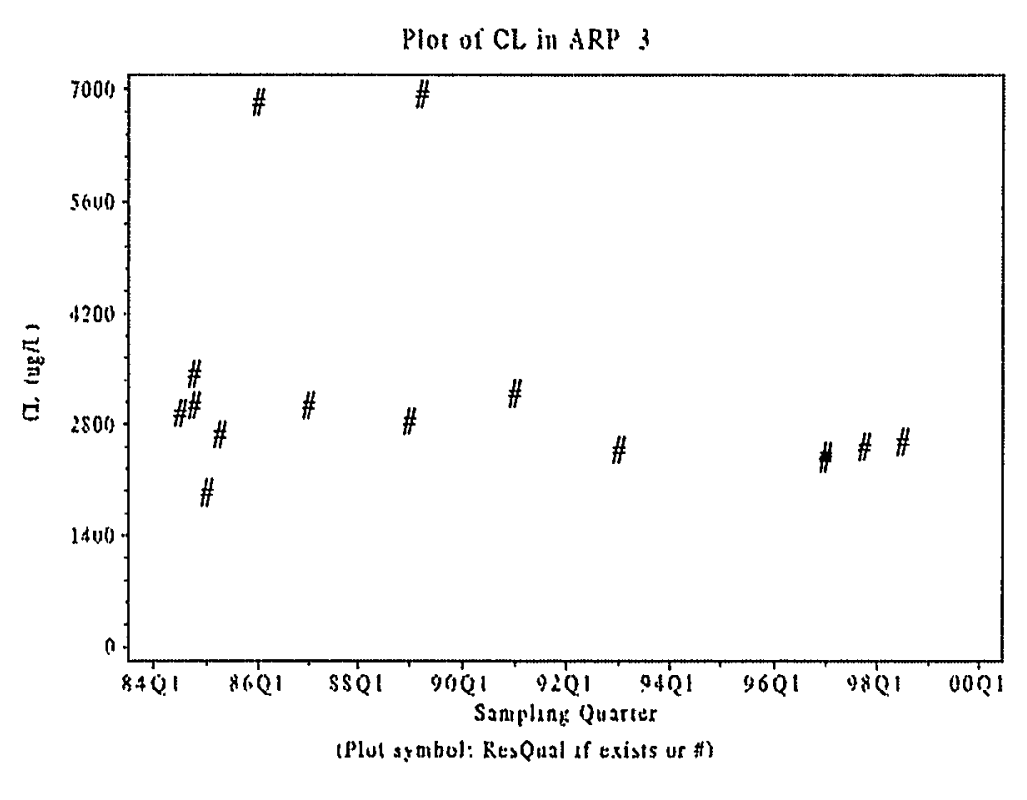


CCL4 Results for ARP 3

Data Retrieved by AWSA v1 from GIMS on 05MAR99 at 12:50

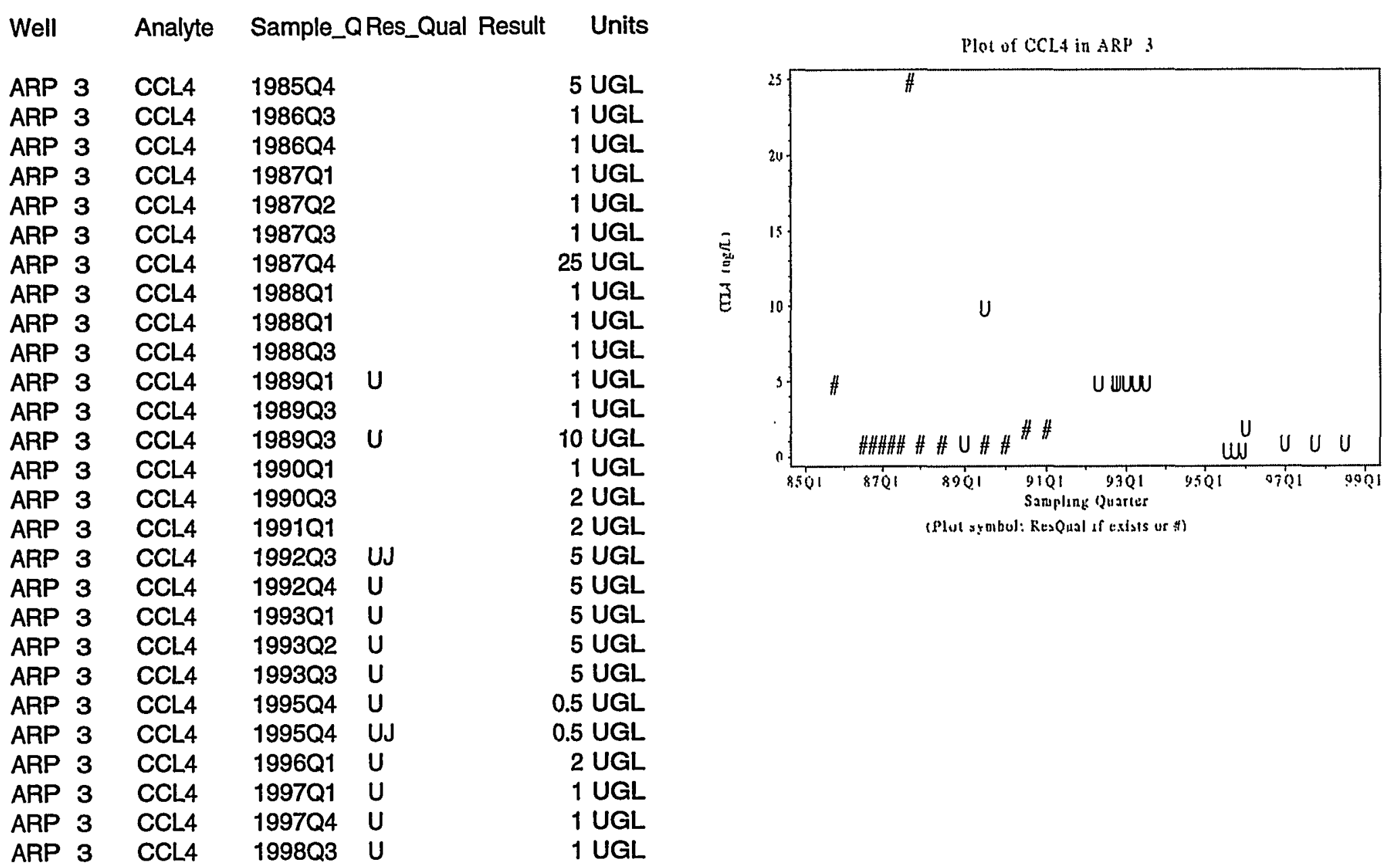


TCLEE Results for ARP 3

Data Retrieved by AWSA v1 from GIMS on 05MAR99 at 12:50

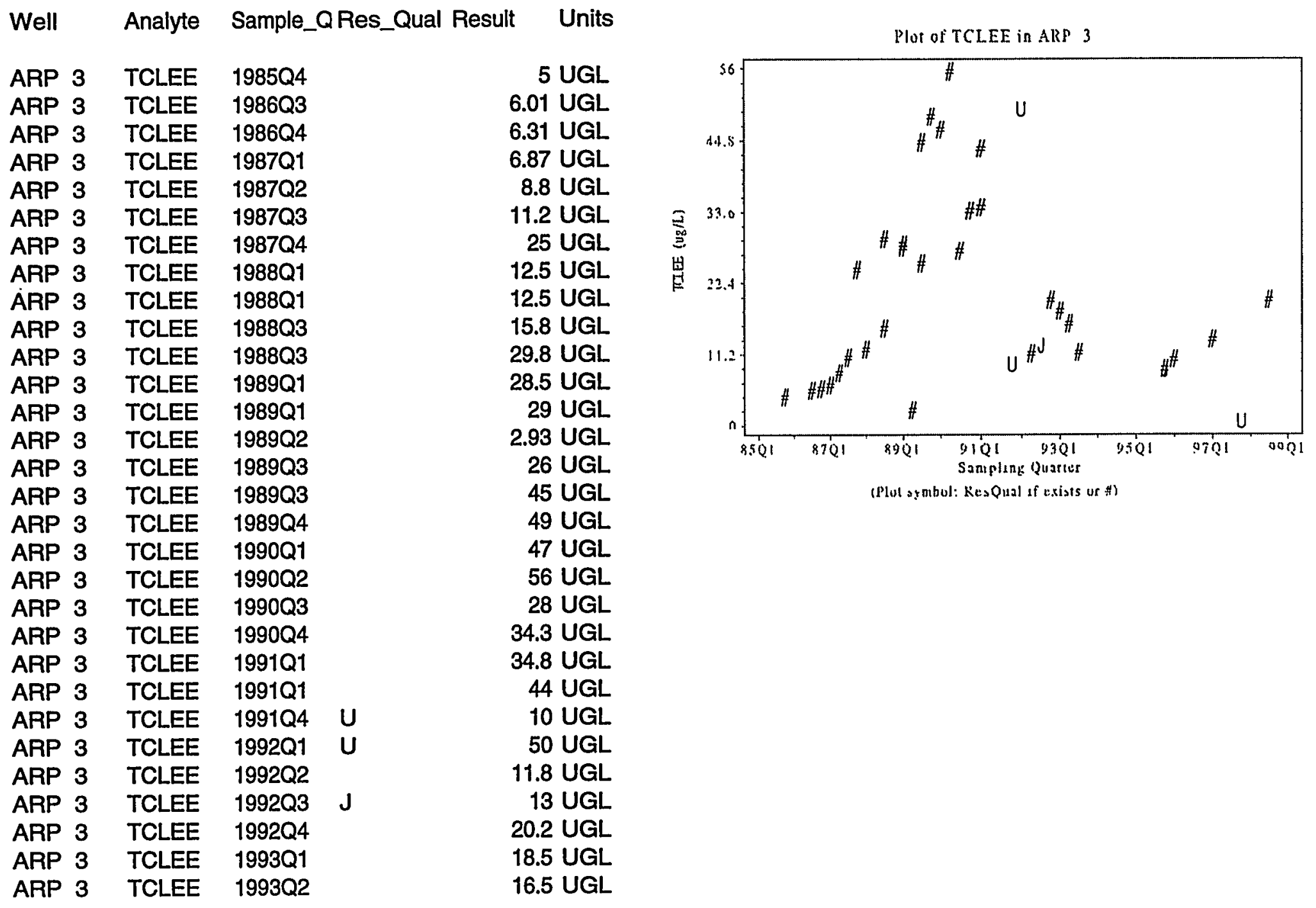


ARP 3 TCLEE 1993 Q 3

ARP 3 TCLEE 1995Q4

ARP 3 TCLEE 1995Q4

ARP 3 TCLEE 1996Q1

ARP $3 \quad$ TCLEE 1997Q1

ARP 3 TCLEE 1997Q4

ARP 3 TCLEE 1998Q3
12 UGL

9.4 UGL

9.1 UGL

10.9 UGL

14 UGL

$1 \mathrm{UGL}$

20.2 UGL 
TRCLE Results for ARP 3

Data Retrieved by AWSA v1 from GIMS on 05MAR99 at 12:50

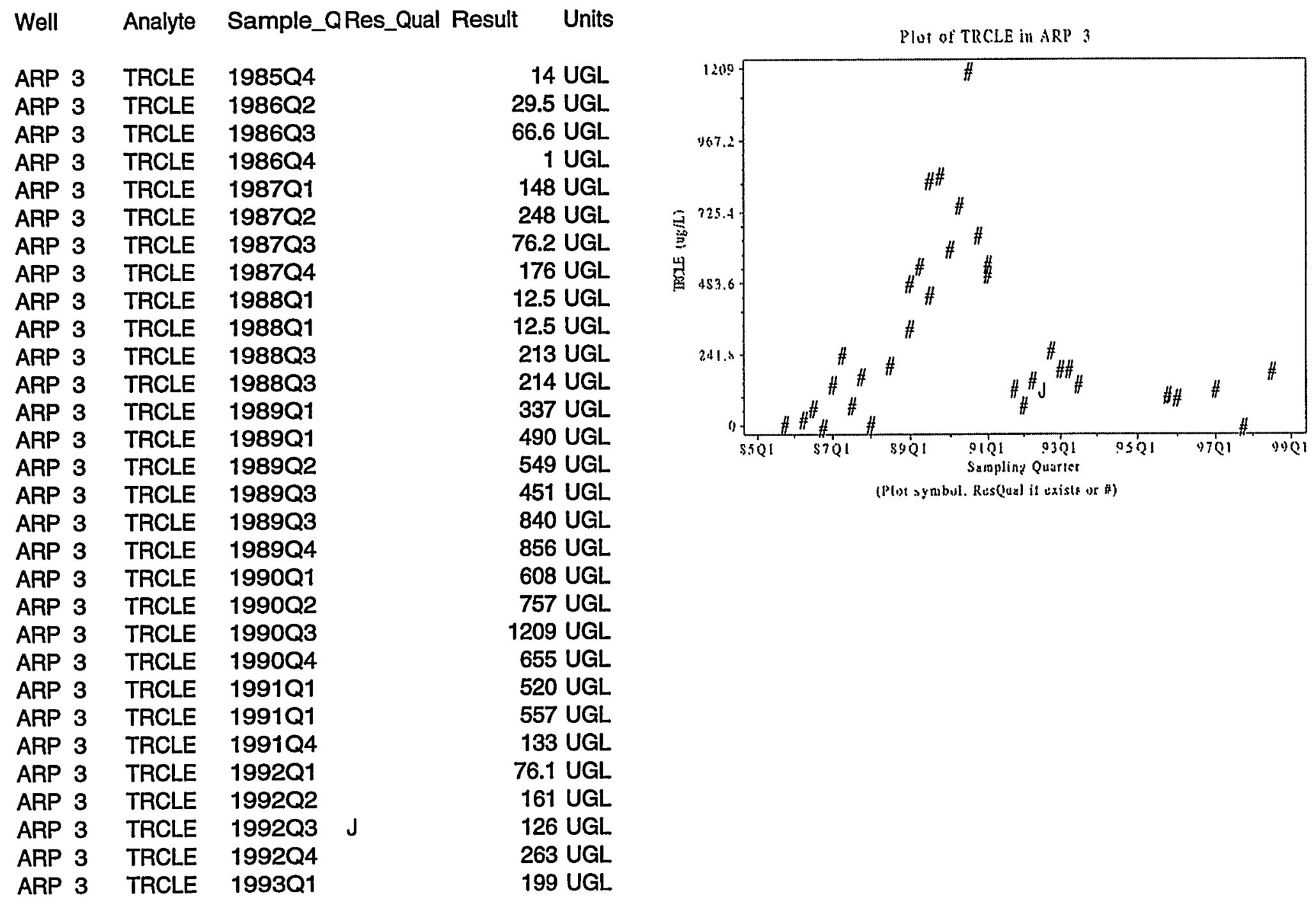


ARP 3 TRCLE 1993Q2

ARP 3 TRCLE 1993Q3

ARP 3 TRCLE 1995Q4

ARP 3 TRCLE 1995Q4

ARP 3 TRCLE 1996Q1

ARP 3 TRCLE 1997Q1

ARP 3 TRCLE 1997Q4

ARP 3 TRCLE 1998Q3

200 UGL

148 UGL

111 UGL

108 UGL

101 UGL

130 UGL

1.02 UGL

191 UGL 


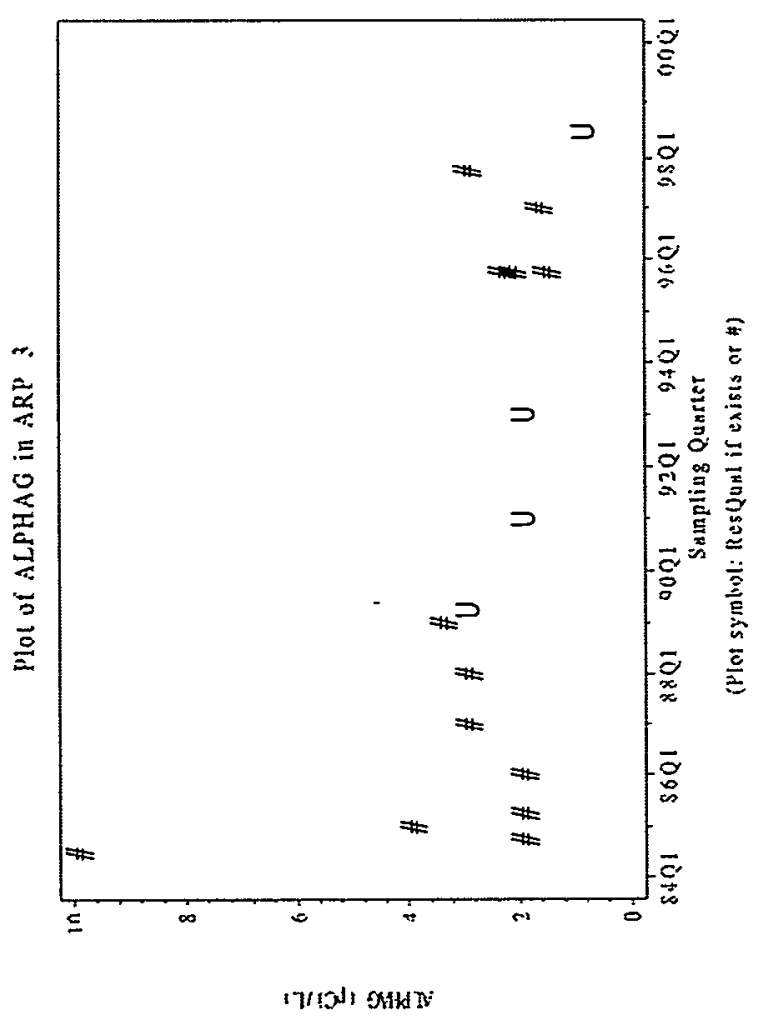

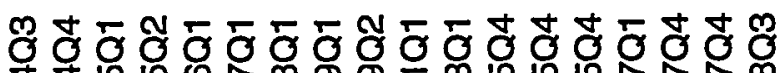

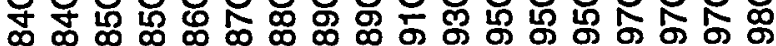

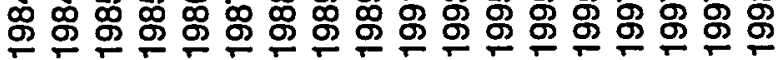

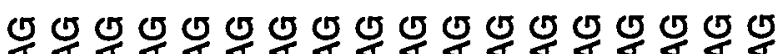

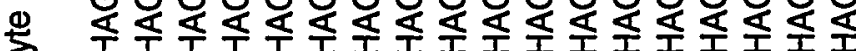

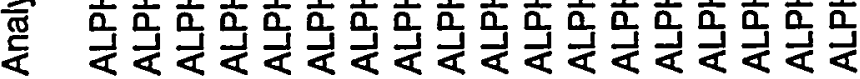

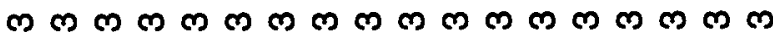

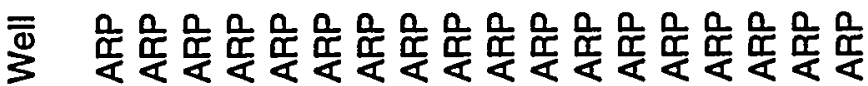


WELL BGO-33C DATA 


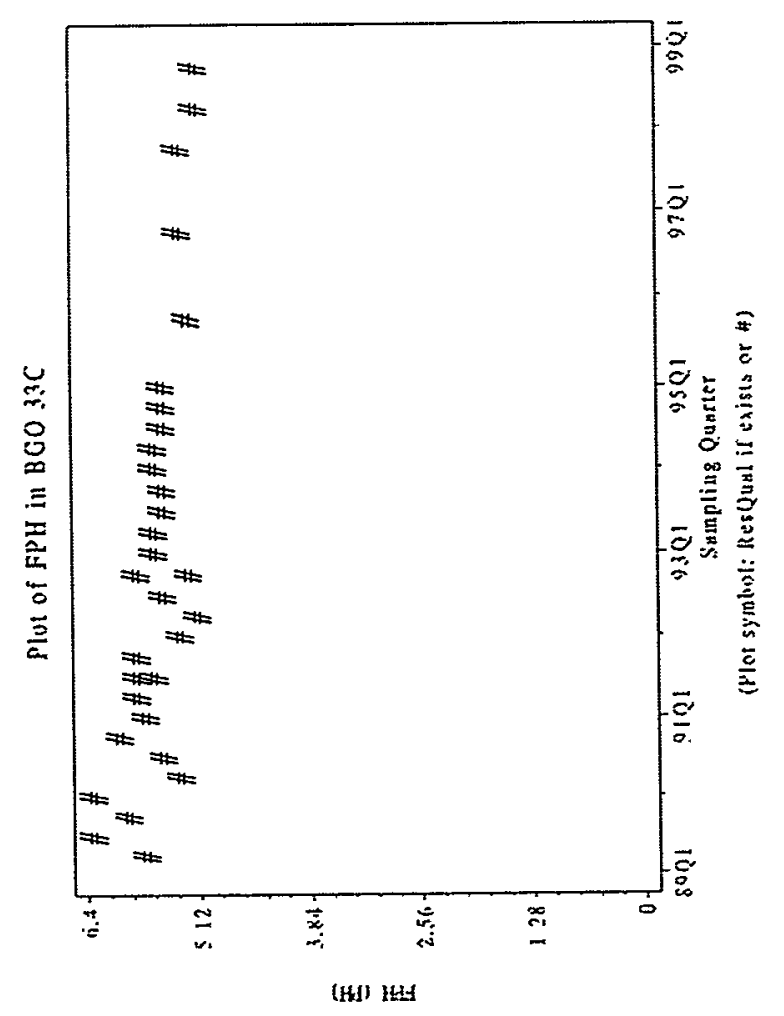

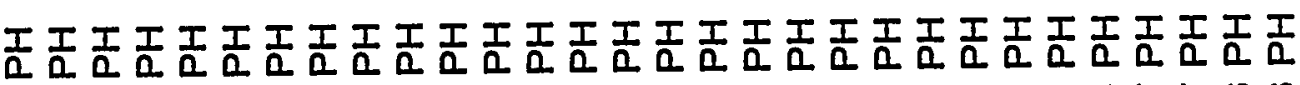

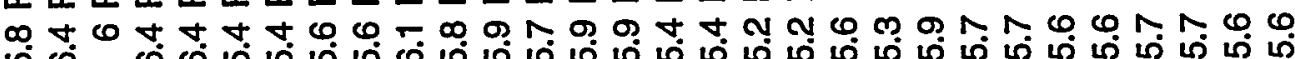
เด

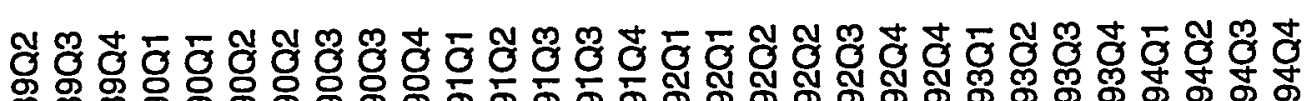
: \% :

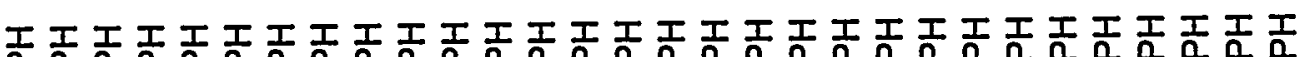

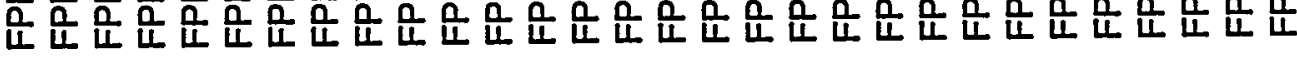

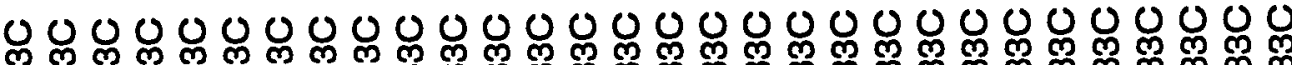
四

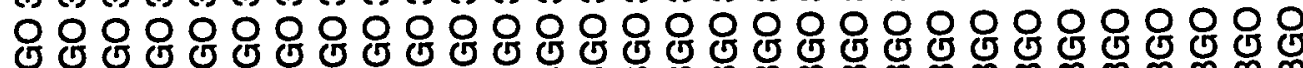

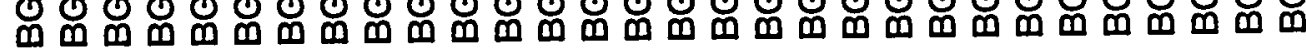


BGO $33 \mathrm{C} \mathrm{FPH}$ BGO $33 \mathrm{C}$ FPH

BGO $33 \mathrm{C} \mathrm{FPH}$

BGO $33 \mathrm{C}$ FPH

BGO $33 \mathrm{C} \mathrm{FPH}$

BGO $33 \mathrm{C}$ FPH

BGO $33 \mathrm{C}$ FPH
1995Q1

1995Q4

1996Q4

1997Q4

1997Q4

1998Q2

1998Q4
5.6 PH

$5.3 \mathrm{PH}$

$5.4 \mathrm{PH}$

$5.4 \mathrm{PH}$

$5.4 \mathrm{PH}$

$5.2 \mathrm{PH}$

$5.2 \mathrm{PH}$ 


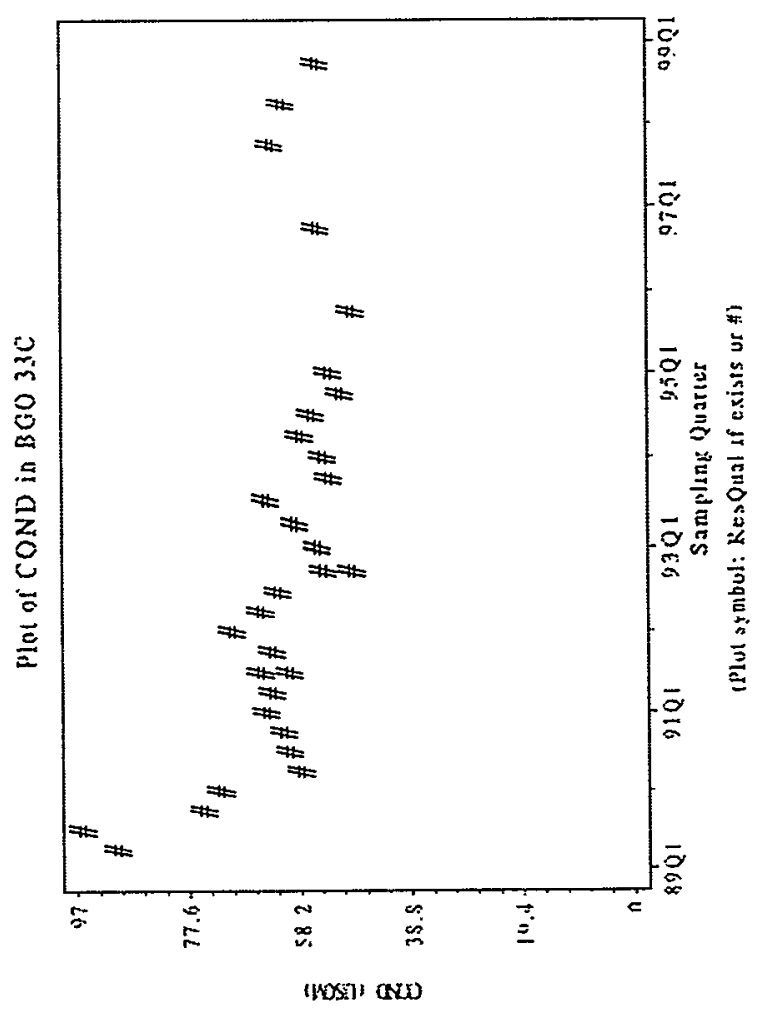

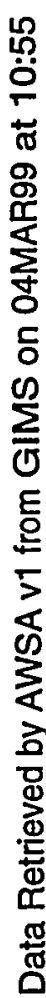

$\stackrel{0}{5}$

$\sum_{0} \sum_{0} \sum_{0} \sum_{0} \sum_{0} \sum_{0} \sum_{0} \sum_{0} \sum_{0} \sum_{0} \sum_{0} \sum_{0} \sum_{0} \sum_{0} \sum_{0} \sum_{0} \sum_{0} \sum_{0} \sum_{0} \sum_{0} \sum_{0} \sum_{0} \sum_{0} \sum_{0} \sum_{0} \sum_{0} \sum_{0} \sum_{0} \sum_{0} \sum_{0}$

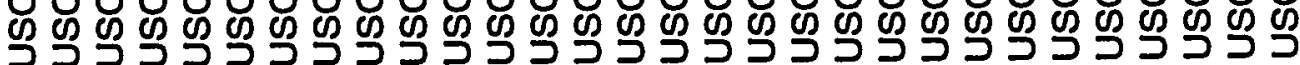

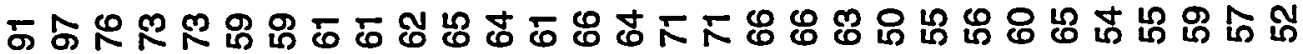

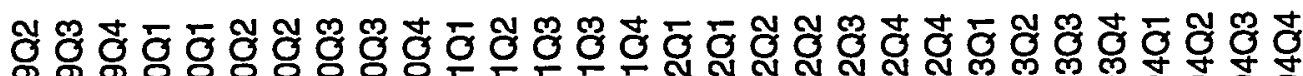
\%

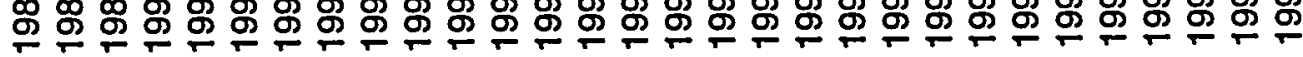

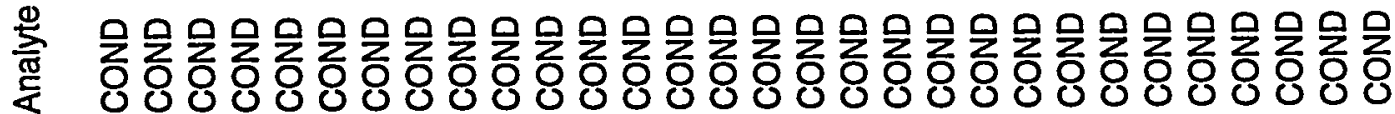
00000000000000000000000000000

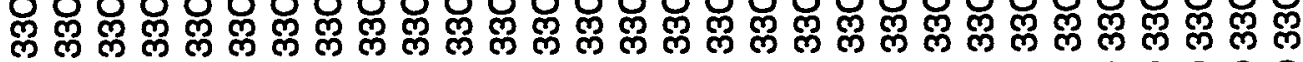

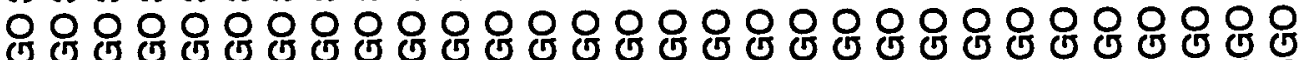

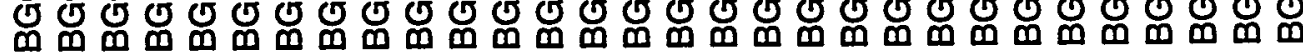




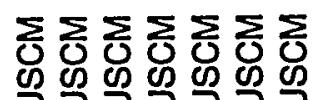

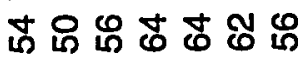

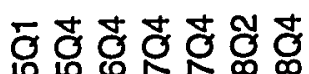

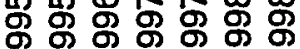

으무우요 우우 우

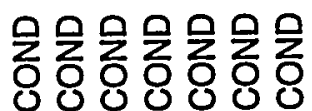

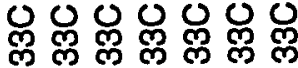
앙엉엉엉ㅇㅇㅇㅇㅇ 品品品品 


\section{CATOT Results for BGO 33C}

Data Retrieved by AWSA v1 from GIMS on 04MAR99 at 14:13

\begin{tabular}{|c|c|c|c|}
\hline Analyte & Sample_ & Res_Qual Result & Units \\
\hline BGO 33 C CATOT & 1992Q4 & & 5090 UGL \\
\hline BGO $33 \mathrm{C}$ САТОT & 1993Q1 & & 4760 UGL \\
\hline BGO 33C CATOT & 1993Q2 & J & 4680 UGL \\
\hline BGO $33 \mathrm{C}$ CATOT & 1993Q3 & & 6740 UGL \\
\hline BGO 33C CATOT & $1993 Q 3$ & & 6910 UGL \\
\hline BGO 33C CATOT & 1993Q4 & & 4610 UGL \\
\hline BGO 33C CATOT & 1994Q1 & & 4240 UGL \\
\hline BGO 33С CATOT & 1994Q2 & & 4370 UGL \\
\hline BGO $33 \mathrm{C}$ CATOT & 1994Q3 & & 4250 UGL \\
\hline BGO 33C CATOT & 1994Q4 & & 4000 UGL \\
\hline BGO 33C CATOT & 1994Q4 & & 4070 UGL \\
\hline BGO 33C CATOT & 1994Q4 & & 4610 UGL \\
\hline BGO $33 \mathrm{C}$ CATOT & 1994Q4 & & 4660 UGL \\
\hline BGO 33С CATOT & 1994Q4 & & 4770 UGL \\
\hline BGO 33C CATOT & 1995Q1 & & 4380 UGL \\
\hline BGO 33C CATOT & 1998Q2 & & 5280 UGL \\
\hline BGO 33C CATOT & 1998Q4 & & 2970 UGL \\
\hline
\end{tabular}

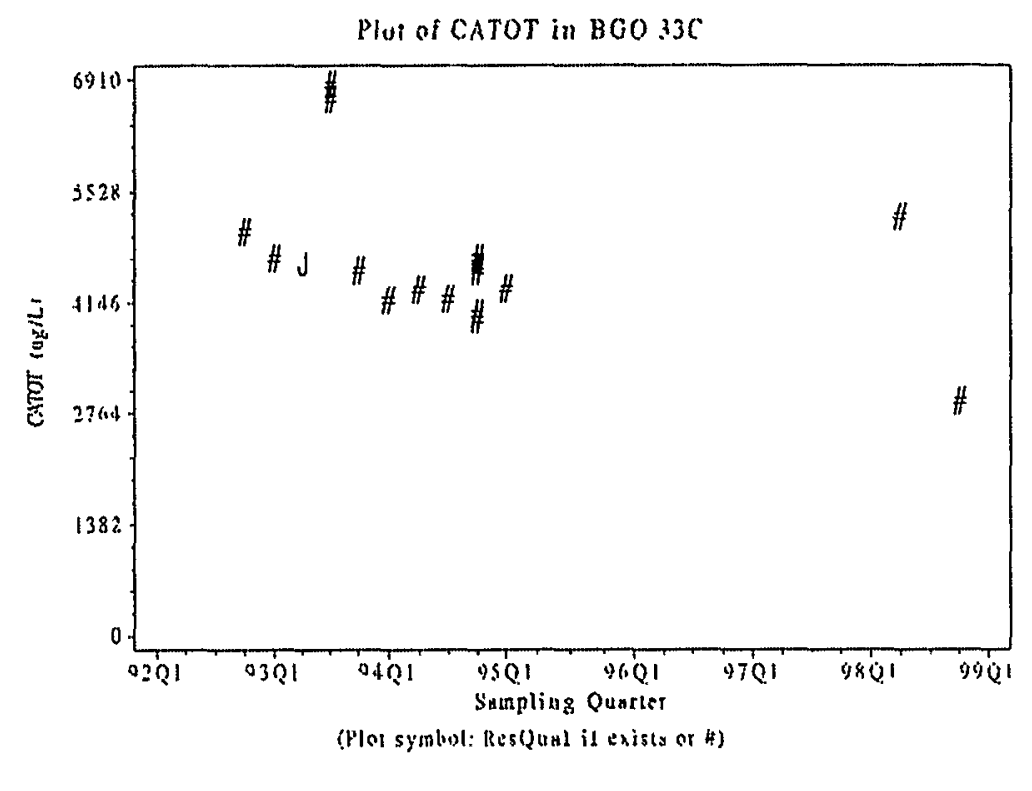


KTOT Results for BGO 33C

Data Retrieved by AWSA v1 from GIMS on 04MAR99 at 14:13

\begin{tabular}{lll} 
Well & Analyte & \multicolumn{2}{c}{ Sample_QRes } \\
& & \\
BGO 33C KTOT & $1992 Q 4$ & $U$ \\
BGO 33C KTOT & $1993 Q 1$ & $U$ \\
BGO 33C KTOT & $1993 Q 2$ & $U$ \\
BGO 33C KTOT & $1993 Q 3$ & \\
BGO 33C KTOT & $1993 Q 3$ & \\
BGO 33C KTOT & $1993 Q 4$ & $U$ \\
BGO 33C KTOT & $1994 Q 1$ & $J$ \\
BGO 33C KTOT & $1994 Q 2$ & $U$ \\
BGO 33C KTOT & $1994 Q 3$ & \\
BGO 33C KTOT & $1994 Q 4$ & $J$ \\
BGO 33C KTOT & $1994 Q 4$ & $U$ \\
BGO 33C KTOT & $1994 Q 4$ & $U$ \\
BGO 33C KTOT & $1994 Q 4$ & $U$ \\
BGO 33C KTOT & $1994 Q 4$ & $U$ \\
BGO 33C KTOT & $1995 Q 1$ & $J$ \\
BGO 33C KTOT & $1998 Q 2$ & \\
BGO 33C KTOT & $1998 Q 4$ &
\end{tabular}

Plot of KTOT in BOO $33 \mathrm{C}$

500 UGL 500 UGL 500 UGL 511 UGL 534 UGL 500 UGL 504 UGL 500 UGL 596 UGL 533 UGL 500 UGL 500 UGL 500 UGL 500 UGL 505 UGL 538 UGL 419 UGL

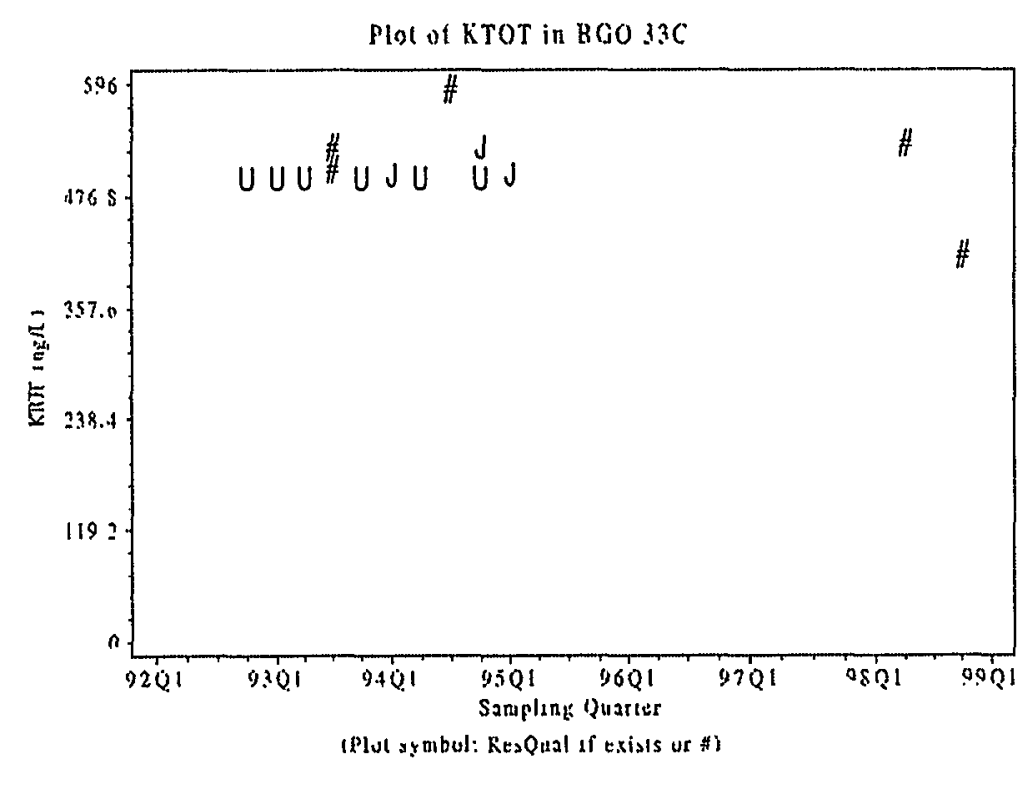


NATOT Results for BGO $33 \mathrm{C}$

Data Retrieved by AWSA v1 from GIMS on 04MAR99 at 14:13

Well Analyte Sample_QRes_Qual Result Units

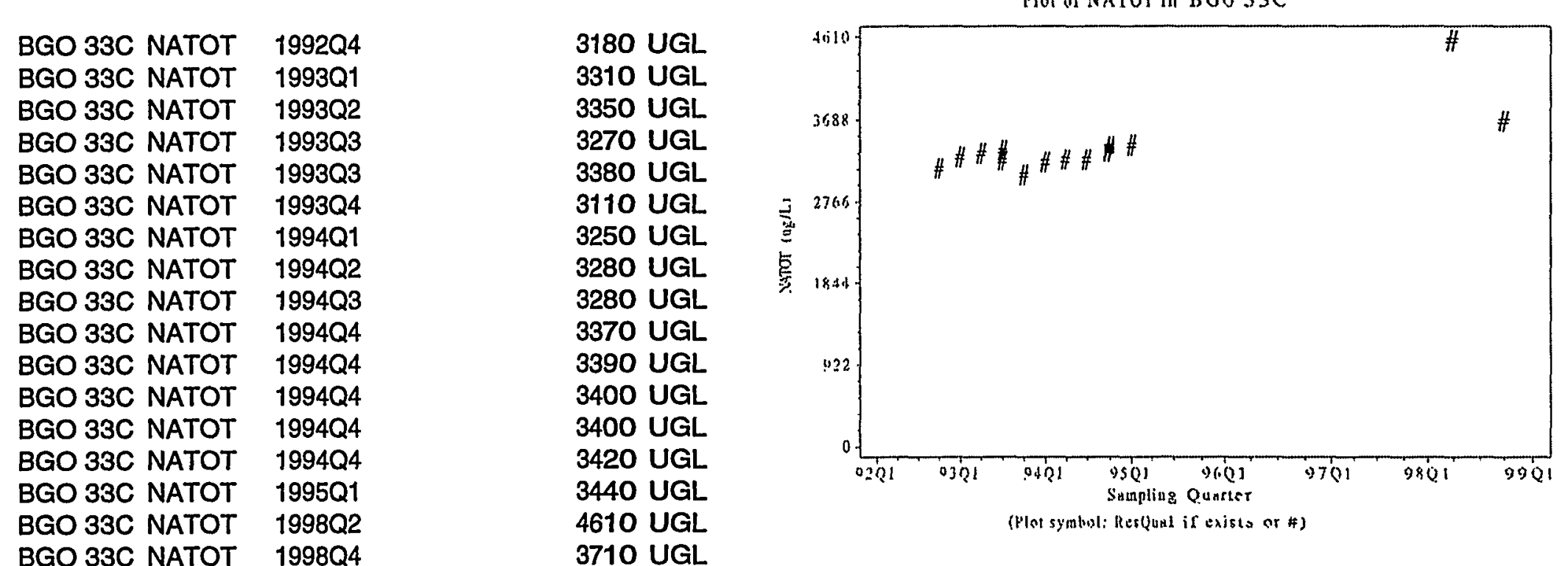


CRTOT Results for BGO 33C

Data Retrieved by AWSA v1 from GIMS on 04MAR99 at 14:13

$\begin{array}{llll}\text { Well } & \text { Analyte } & \text { Sample_QRes_Qual Result } & \text { Units } \\ & & & \\ \text { BGO 33C CRTOT } & 1992 Q 4 & U & 4 \text { UGL } \\ \text { BGO 33C CRTOT } & 1993 Q 1 & U & 4 \text { UGL } \\ \text { BGO 33C CRTOT } & 1993 Q 2 & U & 4 \text { UGL } \\ \text { BGO 33C CRTOT } & 1993 Q 3 & U & 4 \text { UGL } \\ \text { BGO 33C CRTOT } & 1993 Q 3 & U & 4 \text { UGL } \\ \text { BGO 33C CRTOT } & 1993 Q 4 & U & 4 \text { UGL } \\ \text { BGO 33C CRTOT } & 1994 Q 1 & U & 4 \text { UGL } \\ \text { BGO 33C CRTOT } & 1994 Q 2 & U & 4 \text { UGL } \\ \text { BGO 33C CRTOT } & 1994 Q 3 & U & 4 \text { UGL } \\ \text { BGO 33C CRTOT } & 1994 Q 4 & U & 4 \text { UGL } \\ \text { BGO 33C CRTOT } & 1994 Q 4 & U & 4 \text { UGL } \\ \text { BGO 33C CRTOT } & 1994 Q 4 & U & 4 \text { UGL } \\ \text { BGO 33C CRTOT } & 1994 Q 4 & U & 4 \text { UGL } \\ \text { BGO 33C CRTOT } & 1994 Q 4 & U & 4 \text { UGL } \\ \text { BGO 33C CRTOT } & 1995 Q 1 & U & 6.67 \text { UGL } \\ \text { BGO 33C CRTOT } & 1995 Q 4 & J & 1.18 \text { UGL } \\ \text { BGO 33C CRTOT } & 1996 Q 4 & U & 2.29 \text { UGL } \\ \text { BGO 33C CRTOT } & 1997 Q 4 & J & 2.93 \text { UGL } \\ \text { BGO 33C CRTOT } & 1998 Q 2 & J & 2.02 \text { UGL } \\ \text { BGO 33C CRTOT } & 1998 Q 4 & J & 1.46 \text { UGL }\end{array}$

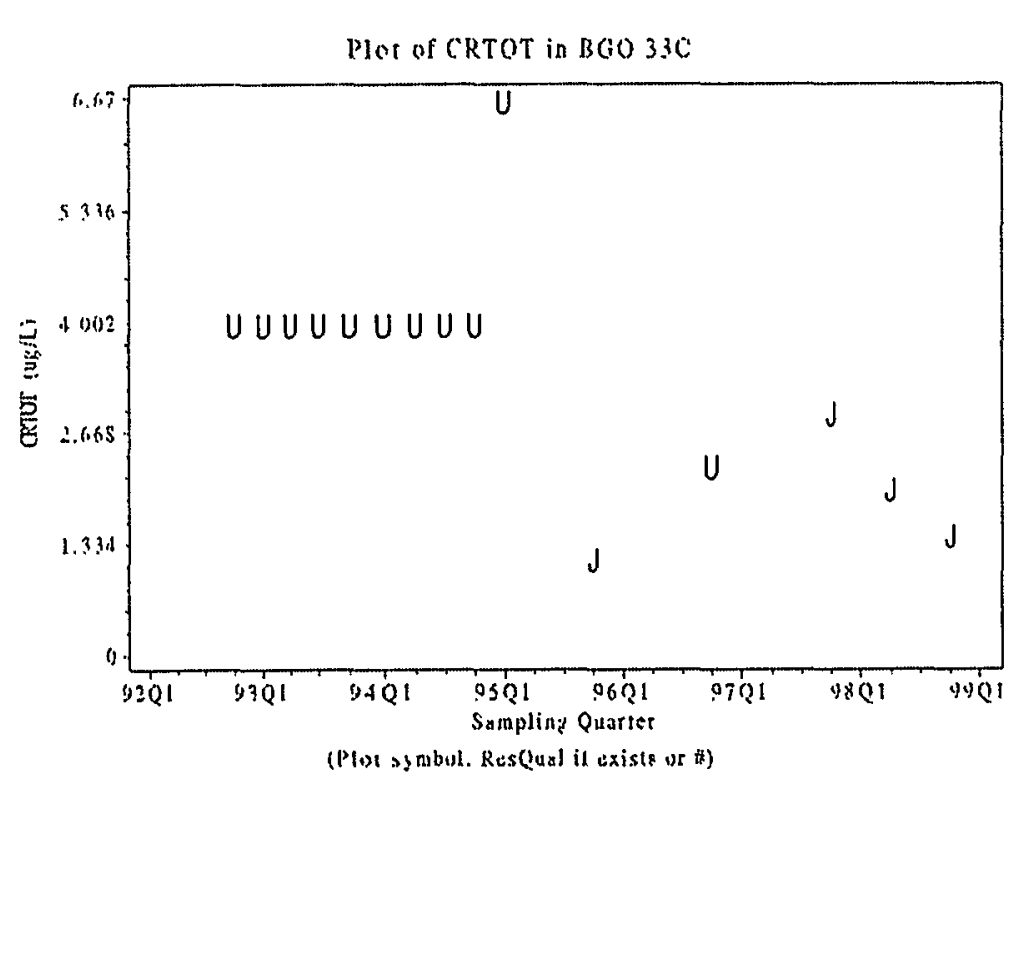


FETOT Results for BGO $33 \mathrm{C}$

Data Retrieved by AWSA v1 from GIMS on 04MAR99 at 14:13

\begin{tabular}{|c|c|c|c|c|}
\hline Well & Analyte & \multicolumn{2}{|c|}{ Sample_QRes_Qual Result } & Units \\
\hline hO $33 \mathrm{C}$ & FETOT & 1992Q4 & & 10.3 UGI \\
\hline GO $33 \mathrm{C}$ & FETOT & 1993Q1 & & 12 UGL \\
\hline GO $33 \mathrm{C}$ & FETOT & 1993Q2 & $U$ & 4 UGL \\
\hline GO $33 \mathrm{C}$ & FETOT & 1993Q3 & & 4.95 UGL \\
\hline GO 33C & FETOT & 1993Q3 & & 5.03 UGL \\
\hline GO $33 \mathrm{C}$ & FETOT & 1993Q4 & & 7.74 UGL \\
\hline $3 \mathrm{GO} 33 \mathrm{C}$ & FETOT & 1994Q1 & & 8.36 UGL \\
\hline $3 \mathrm{GO} 33 \mathrm{C}$ & FETOT & 1994Q2 & $J$ & 6.37 UGL \\
\hline BGO 33C & FETOT & 1994Q3 & & 17.3 UGL \\
\hline BGO $33 \mathrm{C}$ & FETOT & 1994Q4 & & 16.9 UGL \\
\hline BGO 33C & FETOT & 1994Q4 & & 39.3 UGL \\
\hline BGO 33C & FETOT & 1994Q4 & $J$ & 33.8 UGL \\
\hline BGO $33 C$ & FETOT & 1994Q4 & $U$ & 4 UGL \\
\hline BGO 33C & FETOT & 1994Q4 & $U$ & 4 UGL \\
\hline BGO 33C & FETOT & 1995Q1 & $J$ & 5.96 UGL \\
\hline BGO 33C & FETOT & 1998Q2 & $J$ & 32.1 UGL \\
\hline GO $33 C$ & FETOT & 1998Q4 & $U$ & 50 UG \\
\hline
\end{tabular}

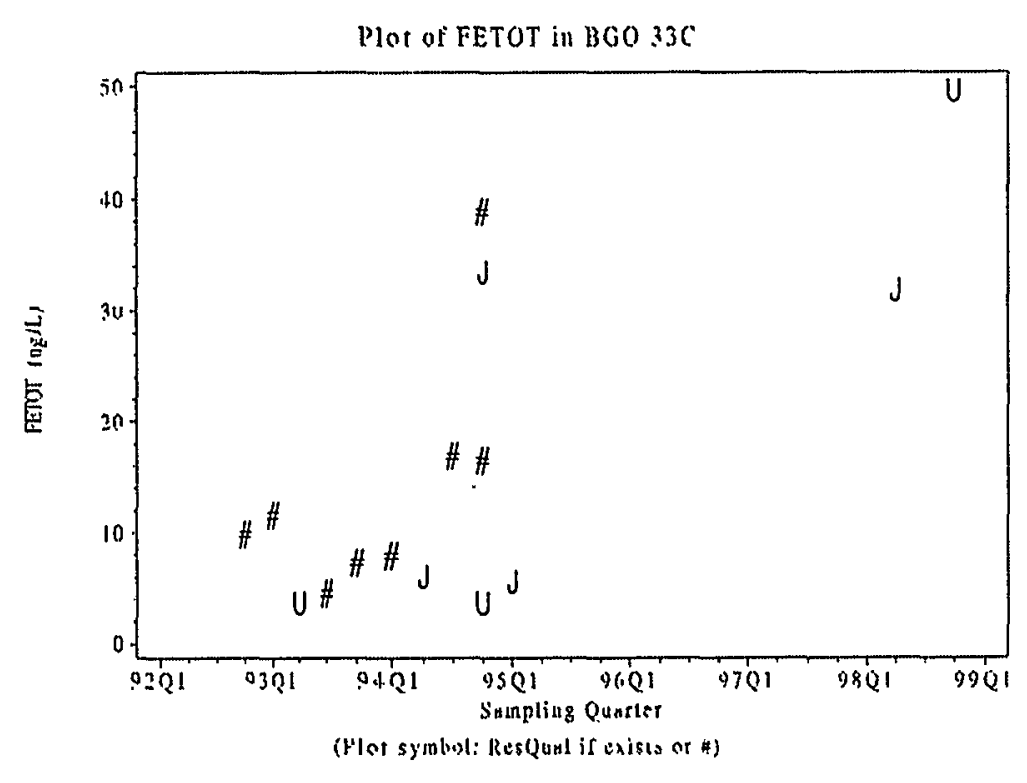


MNTOT Results for BGO 33C

Data Retrieved by AWSA v1 from GIMS on 04MAR99 at 14:13

Well Analyte Sample_QRes_Qual Result Units

BGO 33C MNTOT 1992Q4

BGO 33C MNTOT 1993Q1

BGO 33C MNTOT 1993Q2

BGO 33C MNTOT 1993Q3

BGO 33C MNTOT 1993Q3

BGO 33C MNTOT 1993Q4

BGO 33C MNTOT 1994Q1

BGO 33C MNTOT 1994Q2

BGO 33C MNTOT $1994 Q 3$

BGO 33C MNTOT 1994Q4

BGO 33C MNTOT 1994Q4

BGO 33C MNTOT 1994Q4

BGO 33C MNTOT 1994Q4

BGO 33C MNTOT 1994Q4

BGO 33C MNTOT 1995Q1

BGO 33C MNTOT 1998Q2

BGO 33C MNTOT 1998Q4

31.8 UGL

30.2 UGL

29.2 UGL

32.5 UGL

33.3 UGL

29.3 UGL

29 UGL

30.2 UGL

32.1 UGL

$31.1 \mathrm{UGL}$

31.3 UGL

33.1 UGL

33.6 UGL

33.8 UGL

34 UGL

45.3 UGL

Plot of ANTOT in $B G O, 33 C$

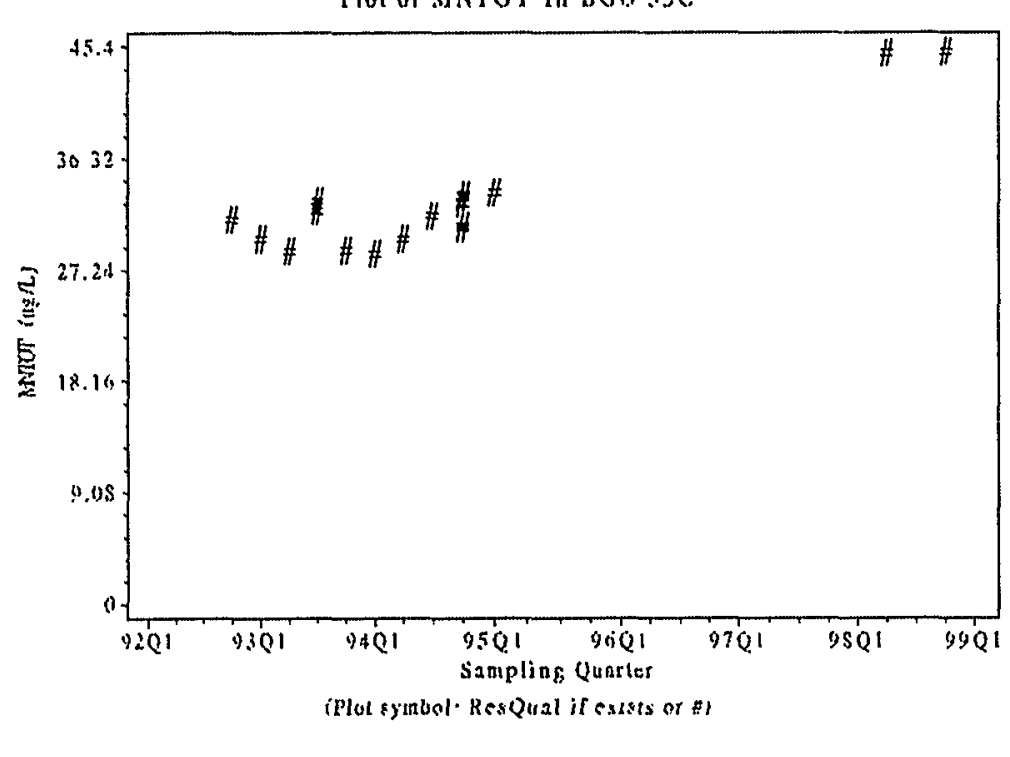


ALTOT Results for BGO 33C

Data Retrieved by AWSA v1 from GIMS on 04MAR99 at 14:13

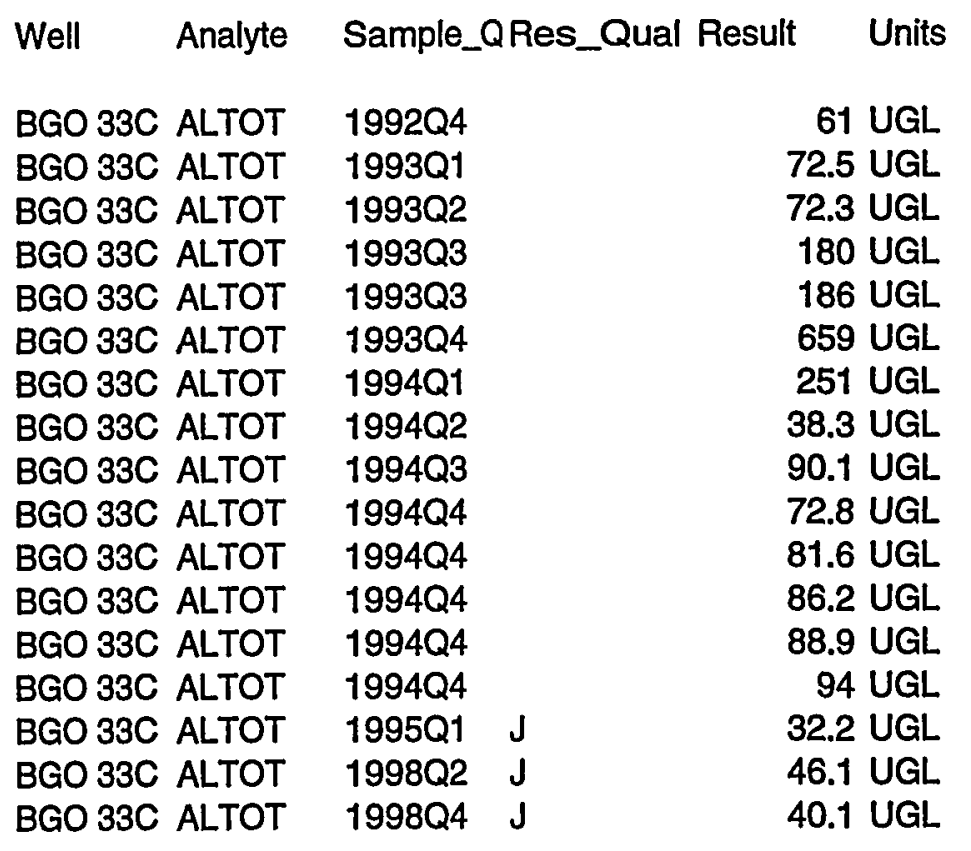

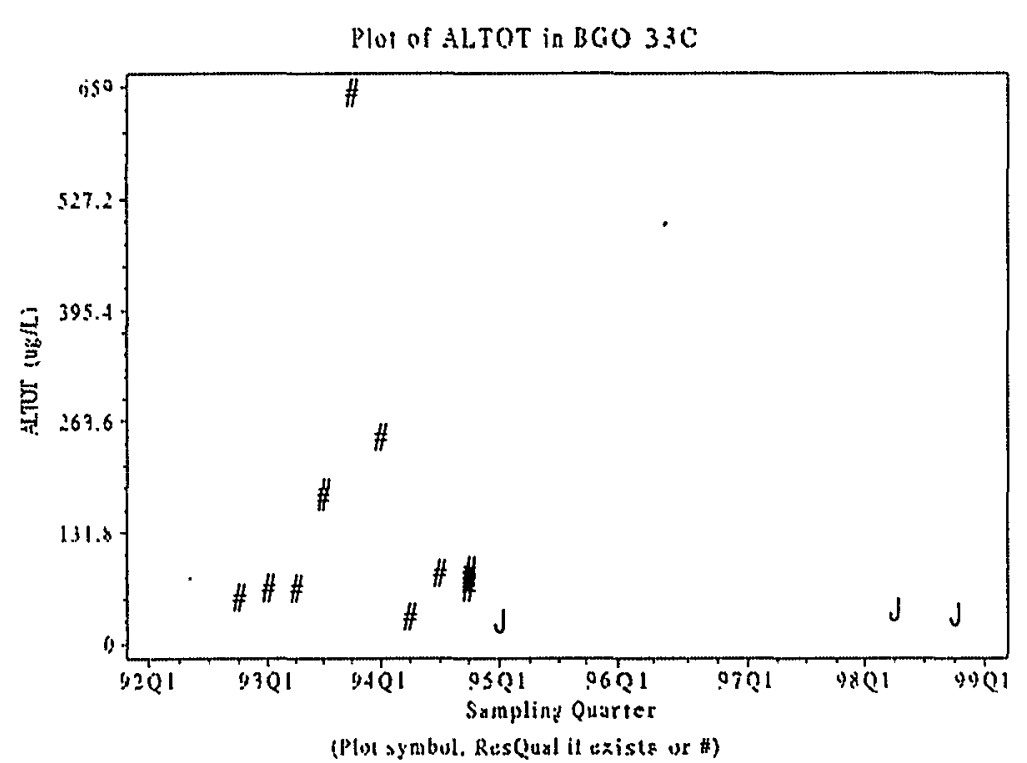


PBTOT Results for BGO 33C

Data Retrieved by AWSA v1 from GIMS on 04MAR99 at 14:13

\begin{tabular}{|c|c|c|c|}
\hline Analyte & \multicolumn{2}{|c|}{ Sample_QRes_Qual Result } & Units \\
\hline BGO 33C PBTOT & 1992Q4 & $u$ & 3 UG \\
\hline BGO 33C PBTOT & 1993Q1 & $\mathbf{U}$ & 3 UGL \\
\hline BGO 33C PBTOT & 1993Q2 & $\mathbf{U}$ & 3 UGL \\
\hline BGO 33C PBTOT & 1993Q3 & $\mathbf{U}$ & 3 UGL \\
\hline BGO 33C PBTOT & 1993Q3 & $\mathbf{U}$ & 3 UGL \\
\hline BGO 33C PBTOT & 1993Q4 & $\mathbf{U}$ & 3 UGL \\
\hline GO 33C PBTOT & 1994Q1 & U & 3 UGL \\
\hline ЗGO $33 \mathrm{C}$ РВTOT & 1994Q2 & U & 3 UGL \\
\hline BGO 33С РВTOT & 1994Q3 & U & 3 UGL \\
\hline BGO 33С РВТOT & 1994Q4 & $\mathrm{U}$ & 3 UGL \\
\hline BGO 33C PBTOT & 1994Q4 & $\mathbf{U}$ & 3 UGL \\
\hline BGO 33C PBTOT & 1994Q4 & $U$ & 3 UGL \\
\hline BGO 33C PBTOT & 1994Q4 & $U$ & 3 UGL \\
\hline BGO 33C РВTOT & 1994Q4 & U & 3 UGL \\
\hline BGO 33C РВTOT & 1995Q1 & U & 5 UGL \\
\hline BGO 33C РВTOT & 1995Q1 & $U$ & 5 UGL \\
\hline BGO 33C PBTOT & 1995Q4 & U & 5 UGL \\
\hline BGO 33C PBTOT & 1996Q4 & $u$ & 2.54 UGL \\
\hline BGO 33C PBTOT & 1997Q4 & $U$ & 5 UGL \\
\hline BGO 33C PBTOT & 1998Q2 & $J$ & 3.07 UGL \\
\hline GO 33С РВTOT & 1998Q4 & U & 5 UG \\
\hline
\end{tabular}

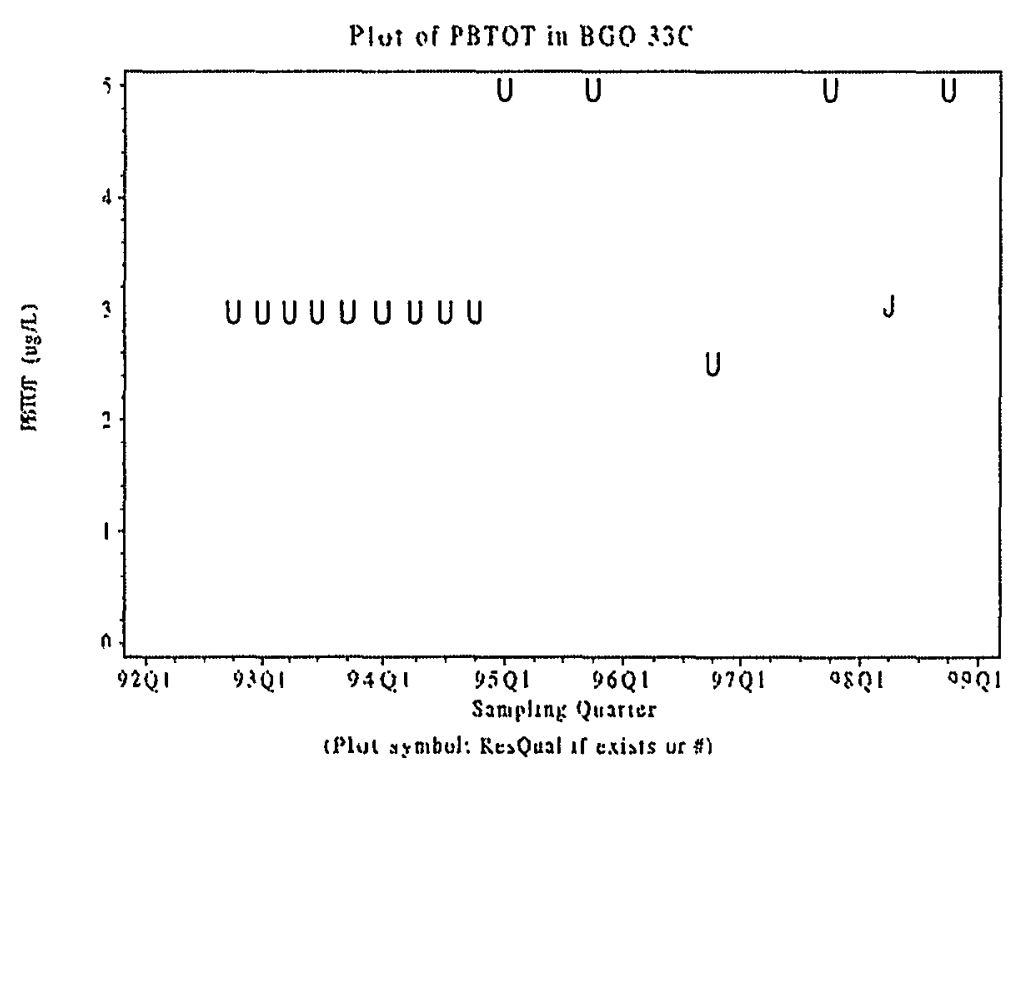




\section{Results for BGO 33C}

Data Retrieved by AWSA v1 from GIMS on 05MAR99 at 12:50

Well Analyte Sample_QRes_Qual Result Units

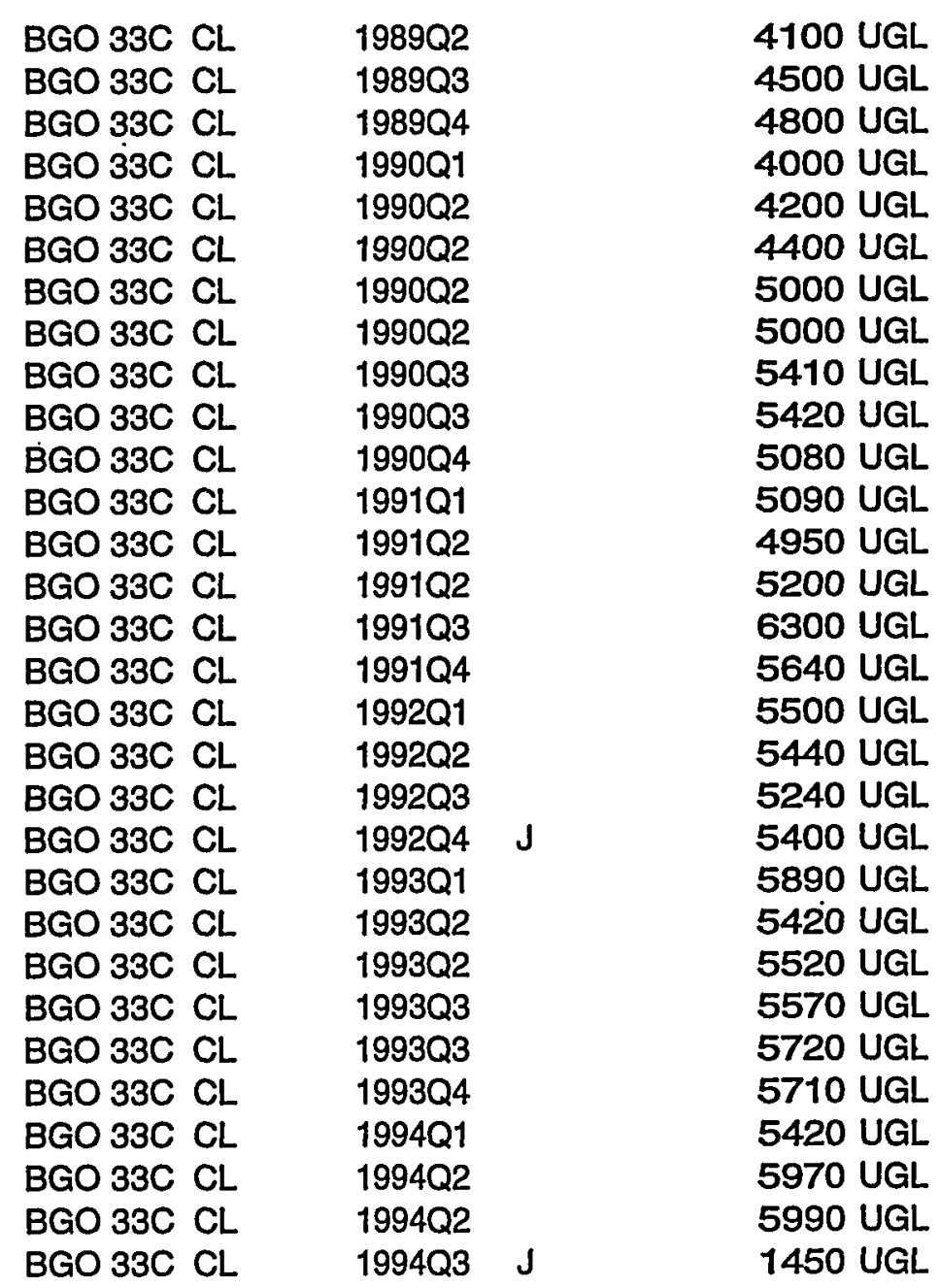

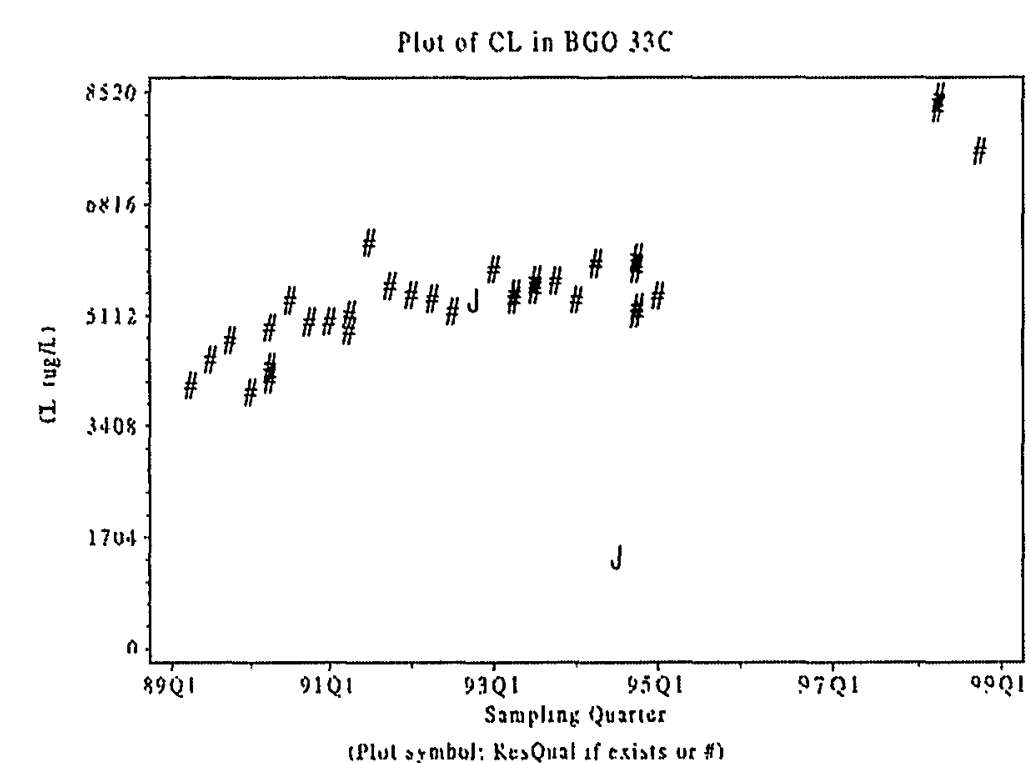




$\begin{array}{lll}\text { BGO 33C CL } & 1994 Q 4 & 5200 \mathrm{UGL} \\ \text { BGO 33C CL } & 1994 Q 4 & 5210 \mathrm{UGL} \\ \text { BGO 33C CL } & 1994 Q 4 & 5300 \mathrm{UGL} \\ \text { BGO 33C CL } & 1994 Q 4 & 5890 \mathrm{UGL} \\ \text { BGO 33C CL } & 1994 Q 4 & 6070 \mathrm{UGL} \\ \text { BGO 33C CL } & 1995 \mathrm{U} 1 & 5480 \mathrm{UGL} \\ \text { BGO 33C CL } & 1998 Q 2 & 8350 \mathrm{UGL} \\ \text { BGO 33C CL } & 1998 Q 2 & 8520 \mathrm{UGL} \\ \text { BGO 33C CL } & 1998 Q 4 & 7700 \mathrm{UGL}\end{array}$


CCL4 Results for BGO 33C

Data Retrieved by AWSA v1 from GIMS on 04MAR99 at 14:13

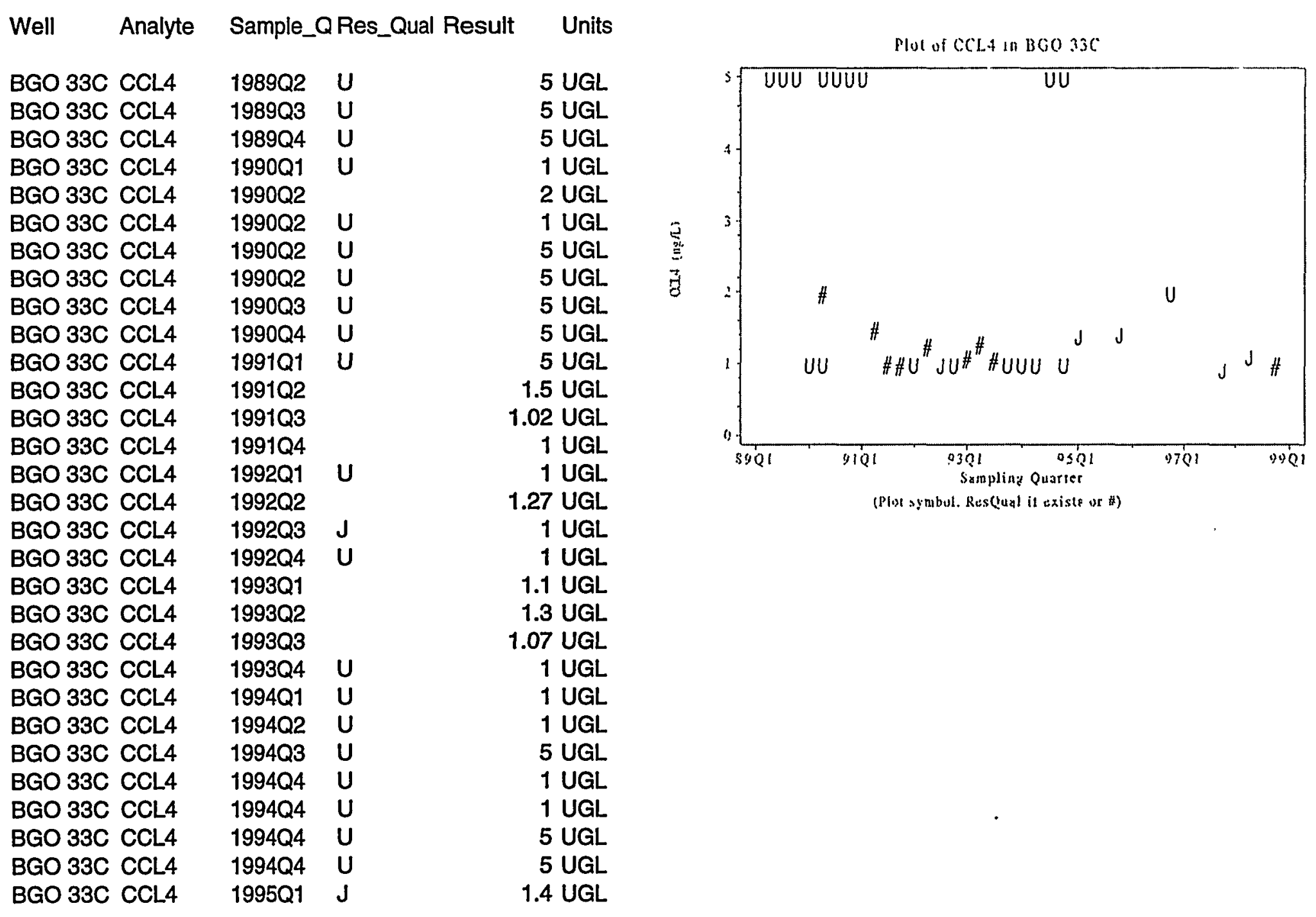


BGO 33C CCL4 $1995 \mathrm{Q} 4$

BGO 33C CCL4 1996Q4 U

BGO 33C CCL4 1997Q4

BGO 33C CCL4 1998Q2

BGO 33C CCL4 1998Q4
1.43 UGL

2 UGL $0.933 \mathrm{UGL}$

$1.11 \mathrm{UGL}$ 1 UGL 
TCLEE Results for BGO 33C

Data Retrieved by AWSA v1 from GIMS on 04MAR99 at 14:13

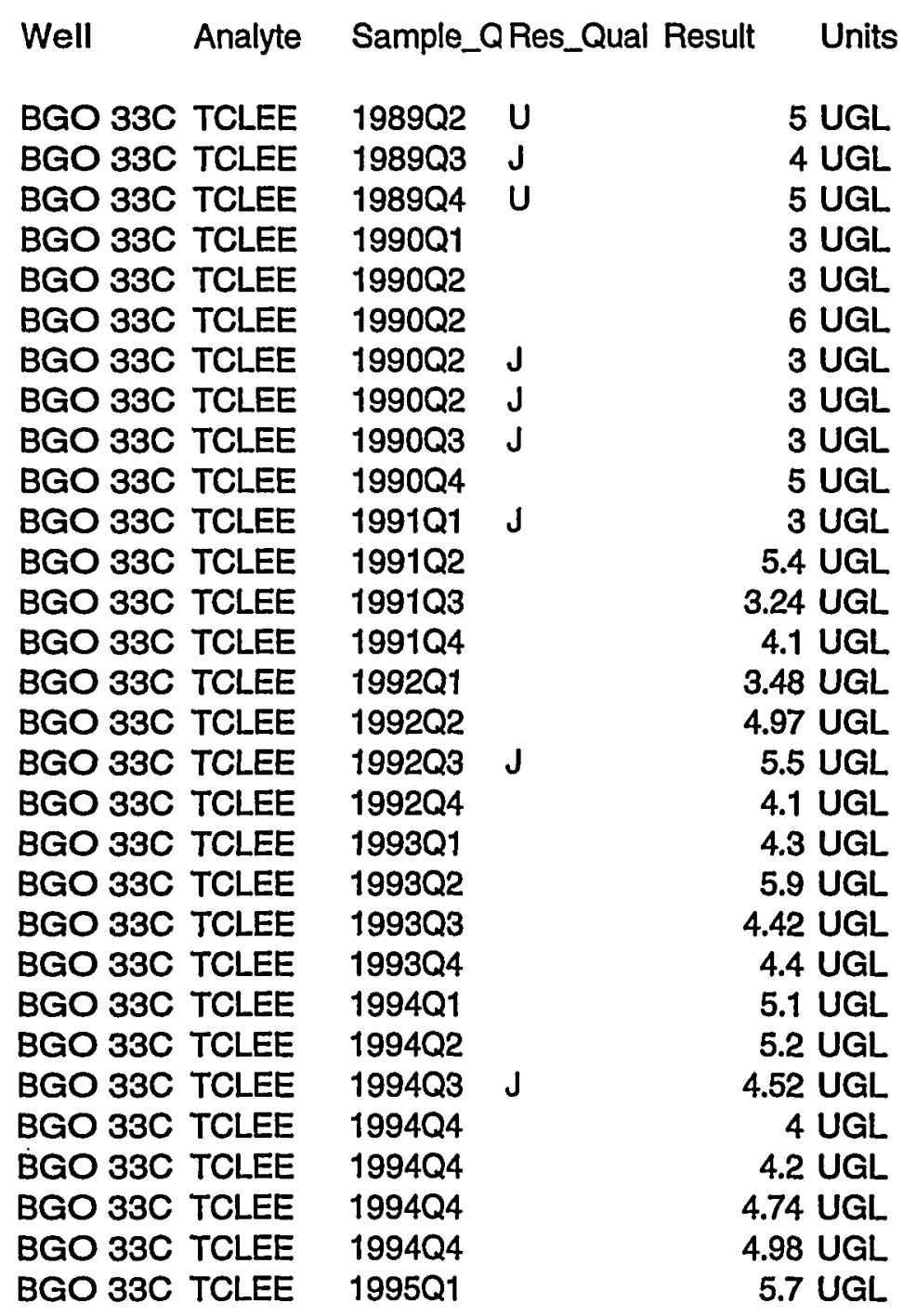

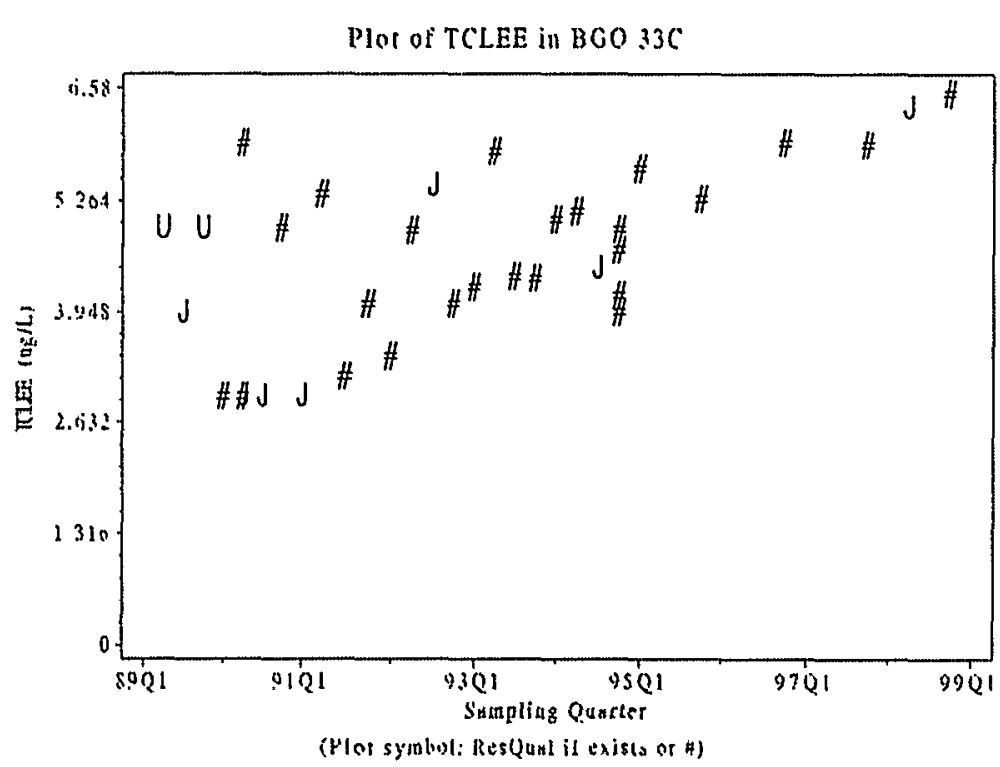


BGO 33C TCLEE 1995Q4

BGO 33C TCLEE 1996Q4

BGO 33C TCLEE 1997Q4

BGO 33C TCLEE 1998Q2

BGO 33C TCLEE 1998Q4
5.34 UGL

6 UGL

5.98 UGL

6.42 UGL

$6.58 \mathrm{UGL}$ 


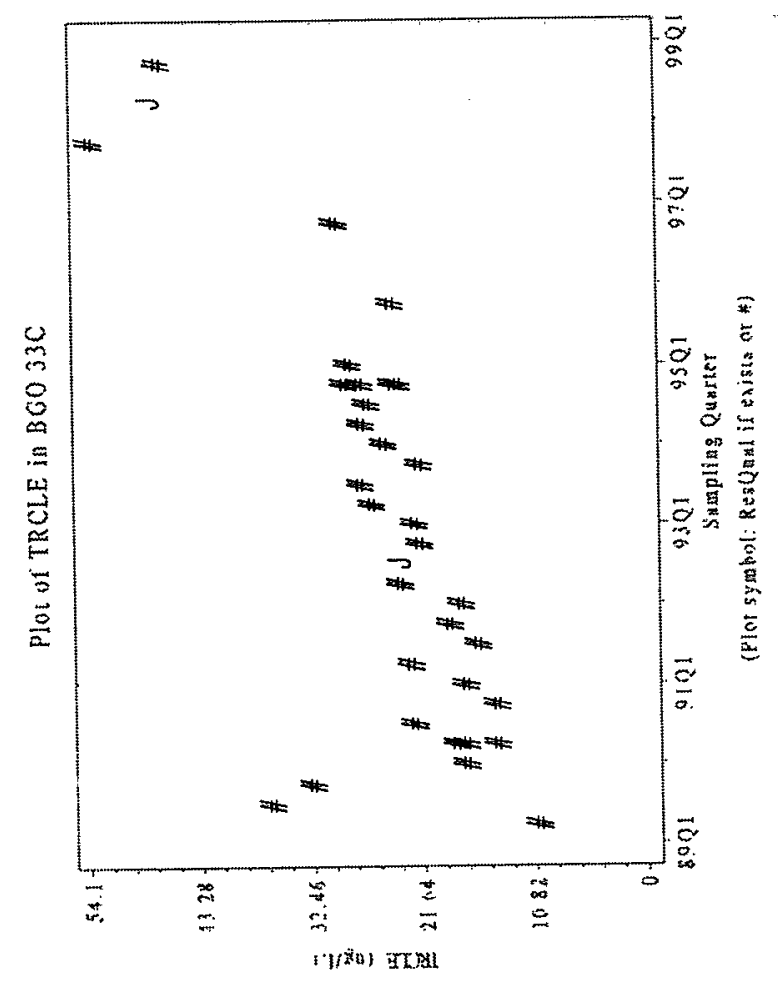

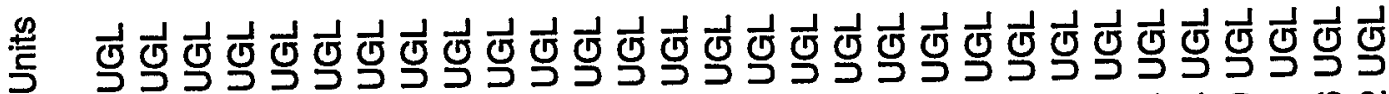

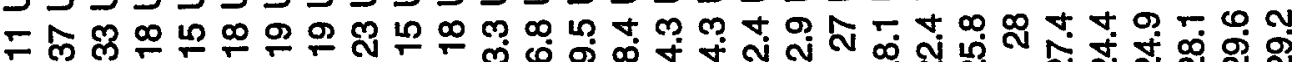

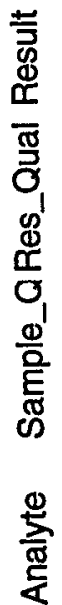
กู่

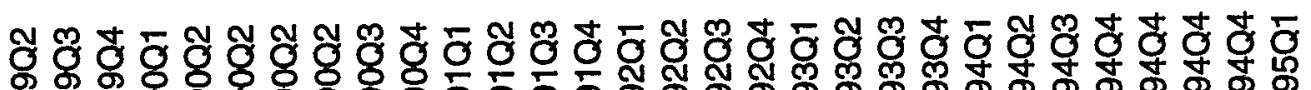

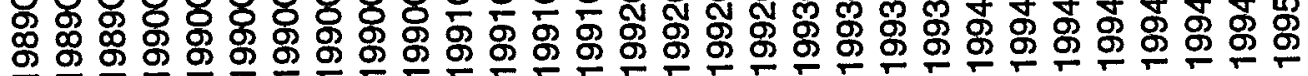

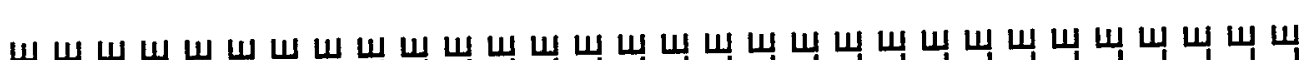

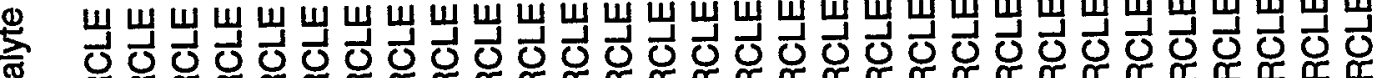

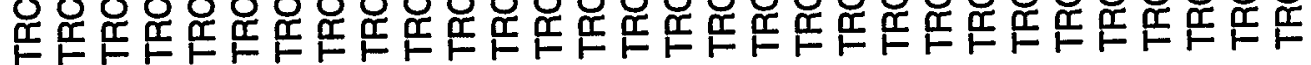

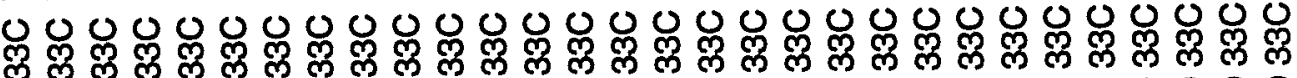

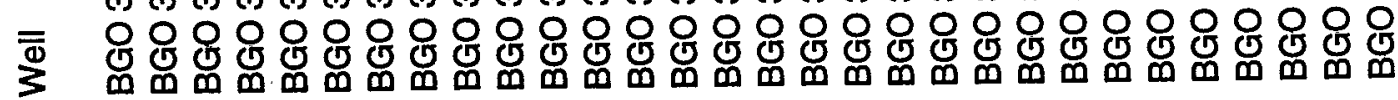


BGO 33C TRCLE 1995Q4

BGO 33C TRCLE 1996Q4

BGO 33C TRCLE 1997Q4

BGO 33C TRCLE 1998Q2

BGO 33C TRCLE 1998Q4
25 UGL

30.4 UGL

54.1 UGL

48.2 UGL

47.4 UGL 
ALPHAG Results for BGO 33C

Data Retrieved by AWSA v1 from GIMS on 04MAR99 at 14:13

Well Analyte Sample_QRes_Qual Result Units

BGO 33C ALPHAG 1989Q2 U

BGO 33C ALPHAG 1989Q3 U

BGO 33C ALPHAG 1989Q4

BGO 33C ALPHAG 1989Q4 U

BGO 33C ALPHAG 1990Q1 U

BGO 33C ALPHAG 1990Q1 U

BGO 33C ALPHAG 1990Q1 U

BGO 33C ALPHAG 1990Q2 U

BGO 33C ALPHAG 1990Q2 U

BGO 33C ALPHAG 1990Q2 U

BGO 33C ALPHAG 1990Q2 U

BGO 33C ALPHAG 1990Q2 U

BGO 33C ALPHAG 1990Q3 U

BGO 33C ALPHAG 1990Q4 U

BGO 33C ALPHAG 1991Q1 U

BGO 33C ALPHAG 1991Q2 U

BGO 33C ALPHAG 1991Q3 U

BGO 33C ALPHAG 1991Q4 U

BGO 33C ALPHAG 1992Q1 U

BGO 33C ALPHAG $1992 Q 2 U$

BGO 33C ALPHAG $199203 \quad U$

BGO 33C ALPHAG 1992Q4 U

BGO 33C ALPHAG 1993Q1 U

BGO 33C ALPHAG 199302

BGO 33C ALPHAG 1993Q3

BGO 33C ALPHAG 1993Q4

BGO 33C ALPHAG 1994Q1 UI

BGO 33C ALPHAG 1994Q2

BGO 33C ALPHAG 1994Q3

BGO 33C ALPHAG 1994Q4
$3 \mathrm{PCL}$
$4 \mathrm{PCL}$
3.4 PCL
$2 \mathrm{PCL}$
$2 \mathrm{PCL}$
$2 \mathrm{PCL}$
$3 \mathrm{PCL}$
$1 \mathrm{PCL}$
$2 \mathrm{PCL}$
$2 \mathrm{PCL}$
$2 \mathrm{PCL}$
$2 \mathrm{PCL}$
$2 \mathrm{PCL}$
$2 \mathrm{PCL}$
$3 \mathrm{PCL}$
$2 \mathrm{PCL}$
2 PCL
2 PCL
$2 \mathrm{PCL}$
$2 \mathrm{PCL}$
$2 \mathrm{PCL}$
$2 \mathrm{PCL}$
2 PCL
$2.04 \mathrm{PCL}$
$2 \mathrm{PCL}$
3.33 PCL
$0.4 \mathrm{PCL}$
2.66 PCL
9.28 PCL

$0.917 \mathrm{PCL}$ 
BGO 33C ALPHAG 1994Q4 J BGO 33C ALPHAG 1994Q4 U BGO 33C ALPHAG 1994Q4 UI BGO 33C ALPHAG 1995Q1 UI BGO 33C ALPHAG 1995Q4 UI BGO 33C ALPHAG 1996Q4 BGO 33C ALPHAG 1997Q4 BGO 33C ALPHAG 1997Q4 BGO 33C ALPHAG 1998Q2 BGO 33C ALPHAG 1998Q4
3.15 PCL

$0 \mathrm{PCL}$

$0.2 \mathrm{PCL}$

$1.09 \mathrm{PCL}$

$0.809 \mathrm{PCL}$

$1.43 \mathrm{PCL}$

$1.13 \mathrm{PCL}$

$1.6 \mathrm{PCL}$

$1.08 \mathrm{PCL}$

$1.73 \mathrm{PCL}$ 
TRITIU Results for BGO 33C

Data Retrieved by AWSA v1 from GIMS on 05MAR99 at 13:25

Well Analyte Sample_QRes_Qual Result Units

\begin{tabular}{|c|c|c|}
\hline BGO 33C TRITIU & 1989Q2 & 4400 PCML \\
\hline BGO 33C TRITIU & 1989Q2 & 4400 PCML \\
\hline BGO 33C TRITIU & 1989Q2 & 5255.1 PCML \\
\hline BGO 33C TRITIU & 1989Q3 & 5000 PCML \\
\hline BGO 33C TRITIU & 1989Q4 & 5400 PCML \\
\hline BGO 33C TRITIU & 1989Q4 & 6100 PCML \\
\hline BGO 33C TRITIU & 1990Q1 & 570 PCML \\
\hline BGO 33C TRITIU & 1990Q1 & 580 PCML \\
\hline BGO 33C TRITIU & $1990 Q 1$ & 7160 PCML \\
\hline BGO 33C TRITIU & 1990Q2 & 5200 PCML \\
\hline BGO 33C TRITIU & 1990Q2 & 5400 PCML \\
\hline BGO 33C TRITIU & 1990Q2 & 5900 PCML \\
\hline BGO 33C TRITIU & 1990Q2 & 7190 PCML \\
\hline BGO 33C TRITIU & 1990Q2 & 7280 PCML \\
\hline BGO 33C TRITIU & 1990Q3 & 5400 PCML \\
\hline BGO 33C TRITIU & 1990Q4 & 5100 PCML \\
\hline BGO 33C TRITIU & 1991Q1 & 5400 PCML \\
\hline BGO 33C TRITIU & 1991Q2 & 6360 PCML \\
\hline BGO 33C TRITIU & 1991Q3 & 6270 PCML \\
\hline BंGO 33C TRITIU & 1991Q4 & 6840 PCML \\
\hline BGO 33C TRITIU & 1992Q1 & 7570 PCML \\
\hline BGO 33C TRITIU & 1992Q2 & 8020 PCML \\
\hline BGO 33C TRITIU & 1992Q3 & 7400 PCML \\
\hline BGO 33C TRITIU & 1992Q4 & 6890 PCML \\
\hline BGO 33C TRITIU & 1993Q1 & 6790 PCML \\
\hline BGO 33C TRITIU & 1993Q2 & 7220 PCML \\
\hline BGO 33C TRITIU & 1993Q3 & 8170 PCML \\
\hline BGO 33C TRITIU & 1993Q4 & 8010 PCML \\
\hline BGO 33C TRITIU & 1994Q1 & 8200 PCML \\
\hline BGO 33C TRITIU & 1994Q2 & 8780 PCML \\
\hline
\end{tabular}

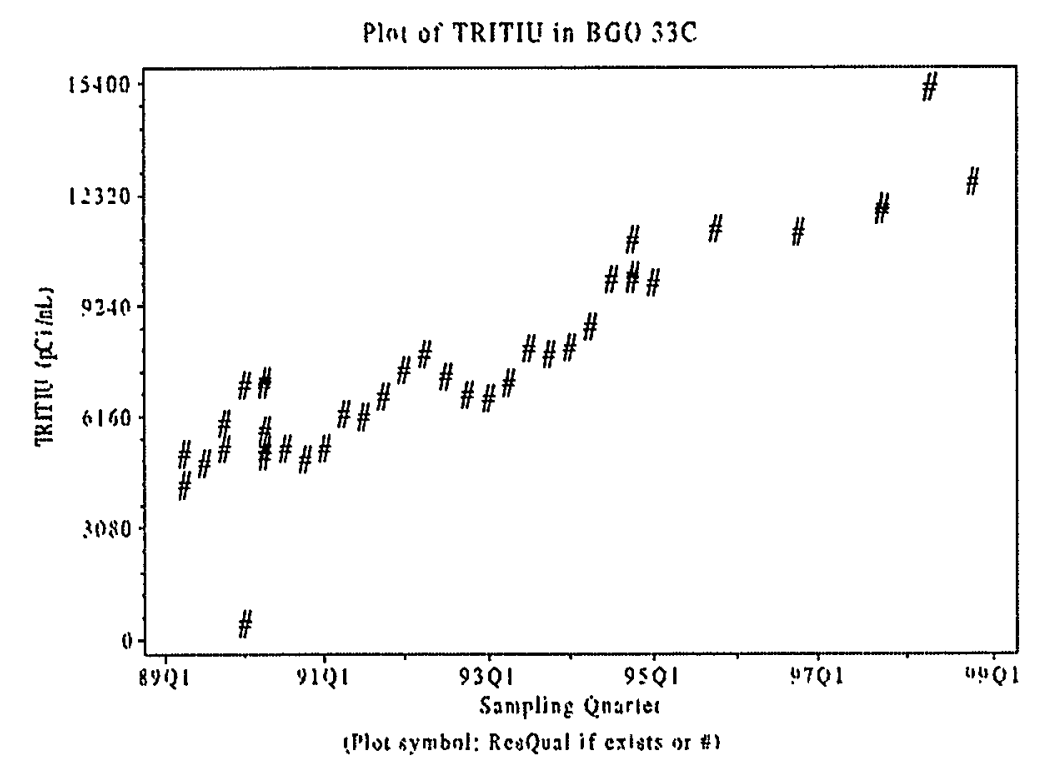


BGO 33C TRITIU 1994Q3

BGO 33C TRITIU 1994Q4

BGO 33C TRITIU 1994Q4

BGO 33C TRITIU 1994Q4

BGO 33C TRITIU 1994Q4

BGO 33C TRITIU 1994Q4

BGO 33C TRITIU 1995Q1

BGO 33C TRITIU 1995Q4

BGO 33C TRITIU 1996Q4

BGO 33C TRITIU 1997Q4

BGO 33C TRITIU 1997Q4

BGO 33C TRITIU 1998Q2

BGO 33C TRITIU 1998Q4
10100 PCML

10100 PCML

$10200 \mathrm{PCML}$

10200 PCML

11200 PCML

11200 PCML

10000 PCML

11500 PCML

11400 PCML

12000 PCML

12100 PCML

15400 PCML

12800 PCML 

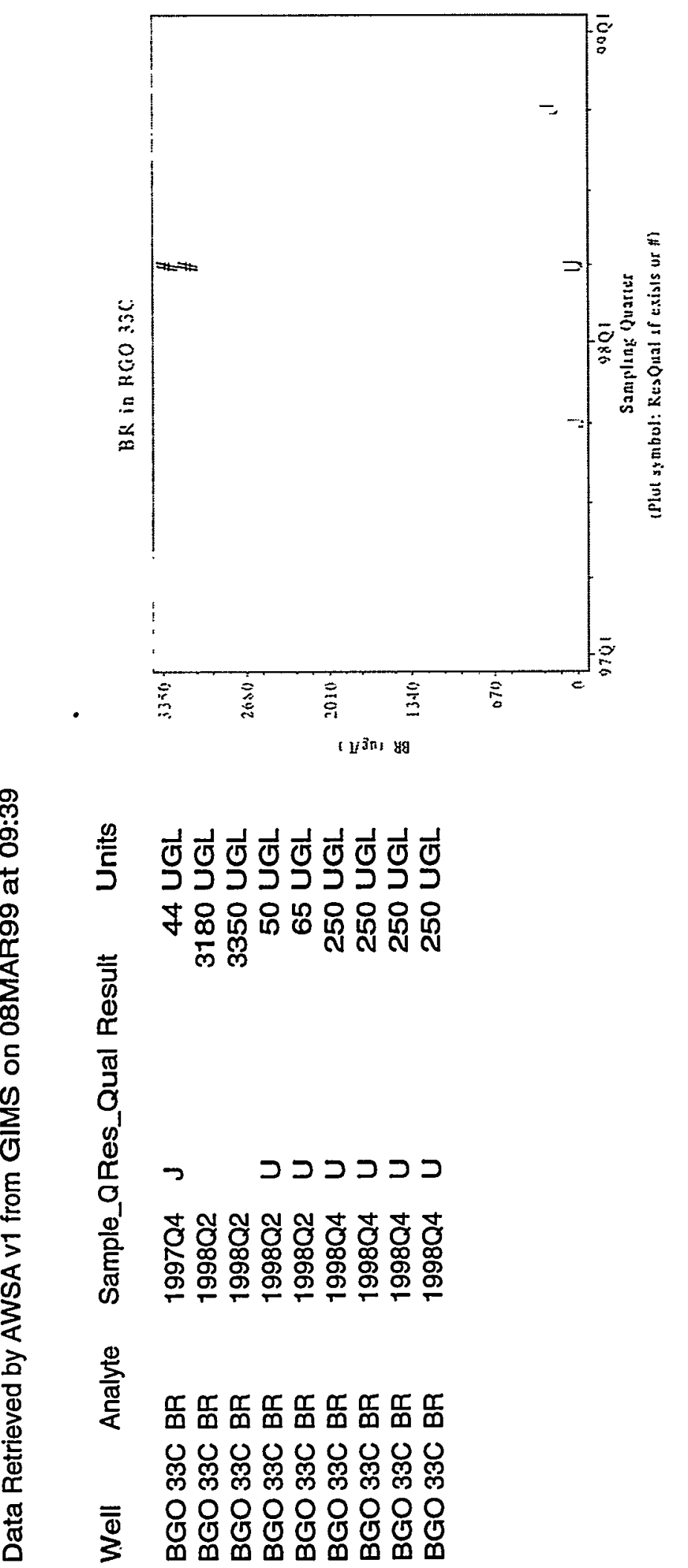


\section{APPENDIX B}

\section{STATISTICAL ANALYSIS OF PWMS GROUNDWATER SAMPLES}




\section{WESTINGHOUSE SAVANNAH RIVER COMPANY Savannah River Technology Center}

SRT-SCS-99-004

February 23, 1999

To:

C.C. Beardsley, 730-2B

From:

S. P. Harris, 773-42A

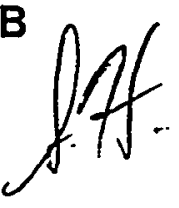

Statistical Analysis of PWMS Groundwater Samples

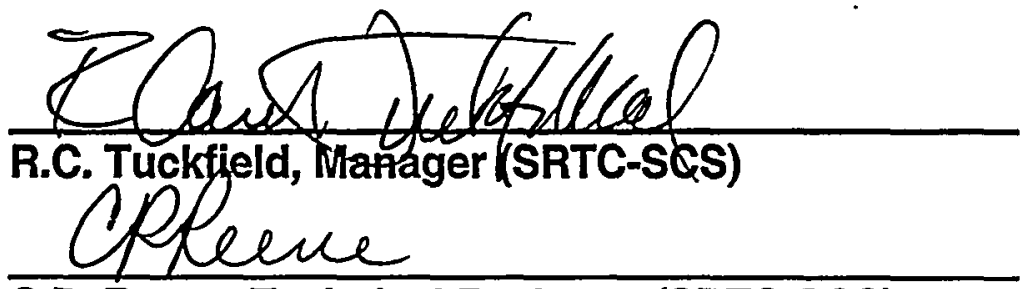

C.P. Reeve, Technical Reviewer (SRTC-SCS)

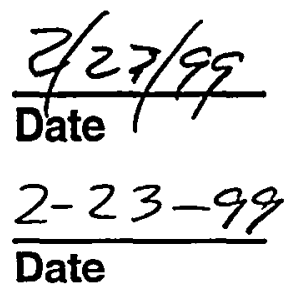

\section{Summary}

A statistical evaluation was done for testing the Purge Water Management System (PWMS) technology using data from routine groundwater monitoring samples of four wells equipped with PWMS tanks in 1997. The data included results from both influent and effluent samples from the PWMS. The wells were designated $A B P$ 1A, ABP 4, ARP 3 and $B G O 33 C$. There was no statistical difference in before or after means for ABP $1 A$, ABP 4 and ARP 3 with $95 \%$ confidence. However, there was a statistical difference with $95 \%$ confidence between the before and after means for BGO 33C. This difference can be traced to five of the fifteen analytes under study. As a result, the influent data were examined for these five analytes. Statistical analysis indicates that only one analyte (total recoverable Manganese) remains problematic. Both practical importance as well as statistical significance should be used in interpreting the statistical results.

\section{Data}

Data were extracted from the GIMS database on analytes for the following wells: ABP 1A, ABP 4 and ARP 3 and BGO 33C. The data included results from both influent and effluent samples from the PWMS. The results collected on the same day were averaged to eliminate serial correlation in the data and the resulting 
data were treated as independent. Data from up to four sampling days were used for the before data if available. Non-detectable results were replaced by half the detection limit for the statistical analysis.

\section{Statistical Analysis}

All calculations were done using SAS Ver. 6.12. An F-test was first examined for equality of variance before deciding which $t$-statistic to use. The t-test for equal variances was used if the variances tested to be equal using the before and after data. Likewise, the t-test for unequal variances was used if the variances did not test to be equal using the before and after data. The t-tests are shown in Tables 1a through 1d for wells $A B P 1 A, A B P 4$ and ARP 3 and BGO $33 C$, respectively. The full analyte names are included in Appendix 1 and the data on which these ttests were based are included in Appendix 2.

Over all $k$ analytes, there is no difference between before or after means for $A B P$ $1 \mathrm{~A}, \mathrm{ABP} 4$ and ARP 3 . The individual $p$-values are greater than $\alpha / k=0.05 / 15=0.0033$ for each analyte. Therefore, the collective sets of before and after means are not statistically different with $95 \%$ confidence using the Bonferroni inequality assuming independence between the analytes. However, the before and after values are different for BGO 33C. This difference can be traced to five of the fifteen analytes under study: CATOT, COND, FETOT, MNTOTand NATOT. Also, KTOT was added to the list for consideration. The influent data for these six analytes were gathered from GIMS and shown in Appendix 3. Statistical analysis indicates that only one analyte (total recoverable Manganese) remains problematic (Table 1e). The before and after values for BGO 33C: MNTOT are included in Chart 1.

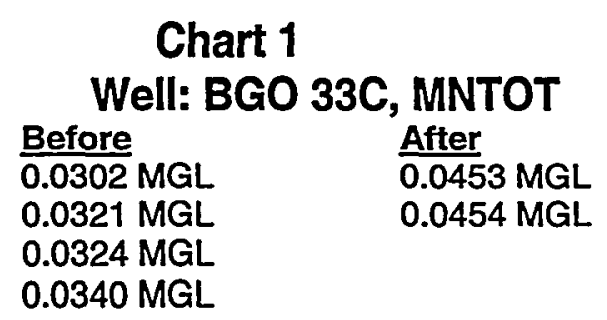

Tables 1a to 1e should be examined for practical importance as well as statistical significance. Typically more data are needed when comparing two groups for equality of means. The power curve in Plot 1 shows that the difference in means would have to be approximately 3 sigma to be. detected a difference with 0.80 probability (power) and $95 \%$ confidence. This represents a best case scenario where the variances are assumed to be equal for both before and after samples and the samples consist of four data points in one sample and two in the other. However, going further back in time was not considered to be practical because of groundwater flow. Additional samples were not available for the "after" data set. 
Table 1a

Well: ABP 1A

PWMS Influent \& Effluent Samples

Analyte

\begin{tabular}{|c|c|c|c|c|c|c|}
\hline$\frac{\text { Code }}{\text { ALPHAG }}$ & $\frac{\text { Units }}{\mathrm{PCL}}$ & $\frac{\text { After }}{1.568}$ & $\frac{\text { Before }}{0.694}$ & $\frac{\mathrm{t} \text {-Test }}{4.73}$ & & $\frac{\mathrm{p}}{0.009^{*}}$ \\
\hline ALTOT & MGL & 0.026 & 0.042 & -0.60 & 3 & 0.59 \\
\hline CATOT & MGL & 0.541 & 0.591 & -0.31 & 2 & 0.78 \\
\hline CCL4 & MGL & 0.0011 & 0.0022 & -1.38 & 4 & 0.24 \\
\hline CL & MGL & 1.607 & 2.104 & -0.61 & 4 & 0.57 \\
\hline COND & USCM & 14.0 & 13.25 & 0.35 & 3 & 0.75 \\
\hline CRTOT & MGL & 0.0029 & 0.0071 & -1.17 & 3 & 0.33 \\
\hline FETOT & MGL & 0.069 & 0.043 & 0.52 & 1 & 0.70 \\
\hline FPH & PH & 4.40 & 5.40 & -0.82 & 3 & 0.47 \\
\hline KTOT & MGL & 0.244 & 0.609 & -0.93 & 1 & 0.52 \\
\hline MNTOT & MGL & 0.0034 & 0.0035 & -0.15 & 4 & 0.89 \\
\hline NATOT & MGL & 0.935 & 1.157 & -1.10 & 2 & 0.38 \\
\hline PBTOT & MGL & 0.0078 & 0.0054 & 0.36 & 4 & 0.74 \\
\hline & MC & 0.0030 & 0.0111 & -2.97 & 4 & $0.041^{*}$ \\
\hline LE & M & 0.0138 & 0.0545 & -3.45 & 4 & $0.026^{*}$ \\
\hline
\end{tabular}

Table 1b

Well: ABP 4

PWMS Influent \& Effluent Samples

Analyte

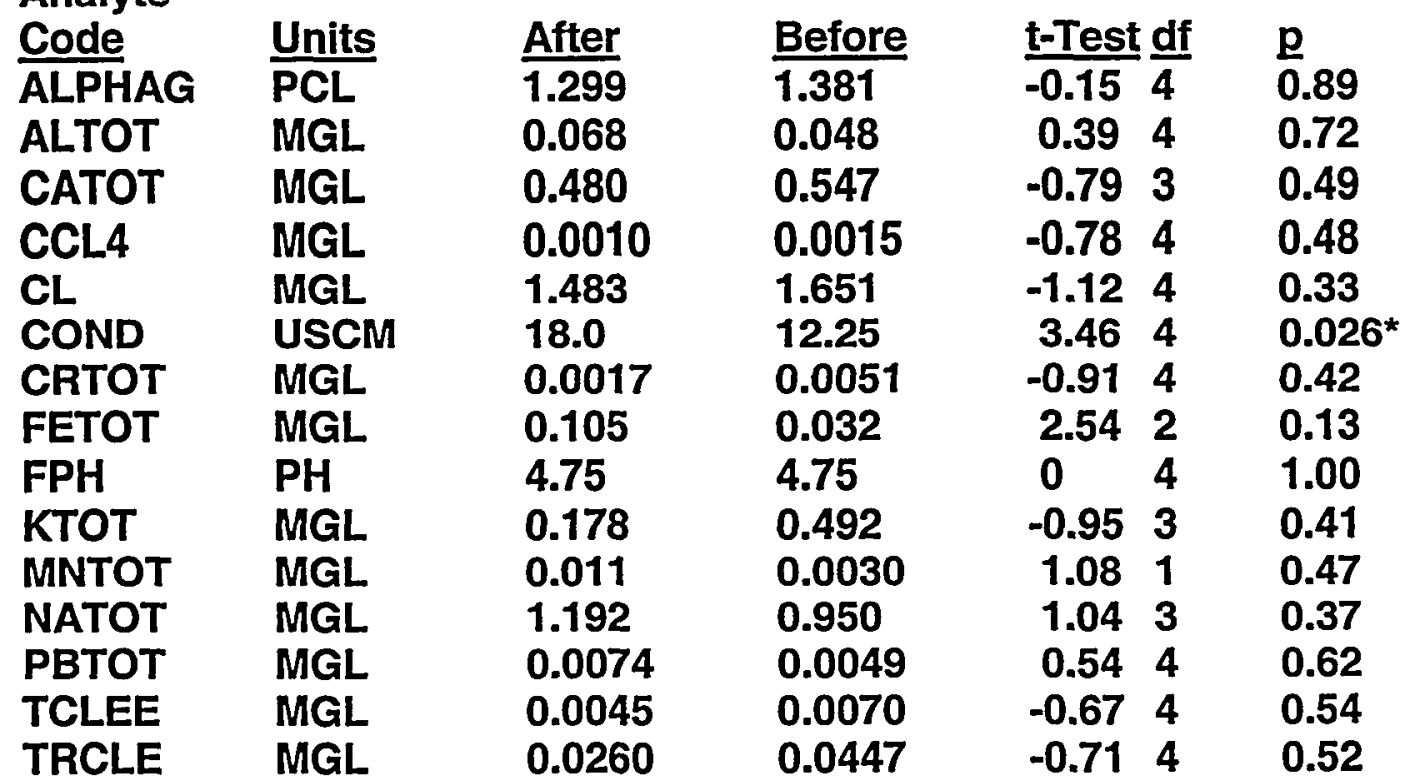

* Differences individually significant at the $5 \%$ level 
Table 1c

Page 4

Well: ARP 3

PWMS Influent \& Effluent Samples

Analyte

\begin{tabular}{llllrll} 
Code & Units & After & Before & t-Test & df & \multicolumn{1}{c}{ p } \\
\cline { 2 - 2 } & PCL & 2.987 & 1.646 & 1.91 & $\frac{0.13}{4}$ & \\
ALTOT & MGL & 0.672 & 0.959 & -0.53 & 3 & 0.63 \\
CATOT & MGL & 0.447 & 0.426 & 0.29 & 3 & 0.79 \\
CCL4 & MGL & 0.00056 & 0.0005 & 0.22 & 4 & 0.83 \\
CL & MGL & 2.603 & 3.810 & -1.12 & 3 & 0.34 \\
COND & USCM & 22.0 & 21.00 & 0.22 & 3 & 0.84 \\
CRTOT & MGL & 0.0114 & 0.0195 & -0.43 & 4 & 0.69 \\
FETOT & MGL & 2.970 & 2.164 & 1.19 & 3 & 0.86 \\
FPH & PH & 4.10 & 4.53 & -1.27 & 3 & 0.29 \\
KTOT & MGL & 0.200 & 0.202 & -0.05 & 3 & 0.96 \\
MNTOT & MGL & 0.0234 & 0.0196 & 0.31 & 4 & 0.78 \\
NATOT & MGL & 2.260 & 1.843 & 2.08 & 3 & 0.13 \\
PBTOT & MGL & 0.0131 & 0.0060 & 1.17 & 4 & 0.31 \\
TCLEE & MGL & 0.0180 & 0.0109 & 3.89 & 4 & $0.018^{*}$ \\
TRCLE & MGL & 0.1748 & 0.1125 & 4.96 & 4 & $0.008^{*}$
\end{tabular}

Table 1d

Well: BGO 33C

PWMS Influent \& Effluent Samples

Analyte

Code

ALPHAG

Units

After

$\frac{\text { Before }}{1378}$

t-Test df $\quad$ p

ALTOT

PCL

1.777

1.378

0.147

0.061

CATOT

CCL4

CL

MGL

5.120

4.339

MGL

0.00194

0.00133

8.629

4.630

63.0

CRTOT

USCM

0.0028

53.00

MGL

0.269

0.0019

MGL

0.011

PH

5.33

FPH

MGL

1.737

MNTOT

MGL

0.0503

5.48

0.418

4.294

PBTOT

MGL

0.0322

3.349

0.0062

0.0019

$\begin{array}{ll}\text { TCLEE } & \text { MGL } \\ \text { TRCLE } & \text { MGL }\end{array}$

0.0061

0.0054

0.0472

0.0278

0.414

0.70

$\begin{array}{lll}2.56 & 3 & 0.08\end{array}$

11.5830 .001 *

$\begin{array}{lll}0.60 & 1 & 0.65\end{array}$

$\begin{array}{lll}1.68 & 3 & 0.19\end{array}$

5.745

$0.002^{*}$

1.204

0.30

37.953

$0.00^{*}$

$-1.355$

0.23

$7.37 \quad 3$

$0.005^{\star}$

10.403

10.363

$0.002^{\star}$

1.151

$0.002^{*}$

1.514

0.46

0.20

$\begin{array}{lll}4.19 & 4 & 0.014\end{array}$

* Differences individually significant at the $5 \%$ level 


\section{Table 1e}

Well: BGO 33C

PWMS Influent \& Effluent Samples(IE)

PWMS Influent Samples Only (I)

IE Statistics were obtained from Table 1d

\begin{tabular}{|c|c|c|c|c|c|}
\hline $\begin{array}{l}\text { Analyte } \\
\text { Code }\end{array}$ & Units & After & Before & $\underline{\mathrm{t} \text {-Test }}$ df & $\mathbf{p}$ \\
\hline $\begin{array}{l}\text { CATOT(IE) } \\
\text { CATOT(I) }\end{array}$ & $\begin{array}{l}\text { MGL } \\
\text { MGL }\end{array}$ & $\begin{array}{l}5.120 \\
4.125\end{array}$ & $\begin{array}{l}4.339 \\
4.339\end{array}$ & $\begin{array}{r}11.583 \\
-0.191\end{array}$ & $\begin{array}{l}0.001^{*} \\
0.883\end{array}$ \\
\hline $\begin{array}{l}\text { COND(IE) } \\
\text { COND(I) }\end{array}$ & $\begin{array}{l}\text { USCM } \\
\text { USCM }\end{array}$ & $\begin{array}{l}63.0 \\
62.0\end{array}$ & $\begin{array}{l}53.00 \\
53.00\end{array}$ & $\begin{array}{ll}5.74 & 5 \\
3.12 & 3\end{array}$ & $\begin{array}{l}0.002^{\star} \\
0.053\end{array}$ \\
\hline $\begin{array}{l}\text { FETOT(IE) } \\
\text { FETOT(I) }\end{array}$ & $\begin{array}{l}\text { MGL } \\
\text { MGL }\end{array}$ & $\begin{array}{l}0.269 \\
0.041\end{array}$ & $\begin{array}{l}0.011 \\
0.011\end{array}$ & $\begin{array}{r}37.953 \\
4.16 \quad 4\end{array}$ & $\begin{array}{l}0.00^{\star} \\
0.014^{*}\end{array}$ \\
\hline $\begin{array}{l}\text { KTOT(IE) } \\
\text { KTOT(I) }\end{array}$ & $\begin{array}{l}\text { MGL } \\
\text { MGL }\end{array}$ & $\begin{array}{l}1.737 \\
0.479\end{array}$ & $\begin{array}{l}0.418 \\
0.418\end{array}$ & $\begin{array}{ll}7.37 & 3 \\
0.48 & 3\end{array}$ & $\begin{array}{l}0.005^{*} \\
0.655\end{array}$ \\
\hline $\begin{array}{l}\text { MNTOT(IE) } \\
\text { MNTOT(I) }\end{array}$ & $\begin{array}{l}\text { MGL } \\
\text { MGL }\end{array}$ & $\begin{array}{l}0.0503 \\
0.0454\end{array}$ & $\begin{array}{l}0.0322 \\
0.0322\end{array}$ & $\begin{array}{l}10.403 \\
11.294\end{array}$ & $\begin{array}{l}0.002^{*} \\
0.0004^{\star}\end{array}$ \\
\hline $\begin{array}{l}\text { NATOT(IE) } \\
\text { NATOT(I) }\end{array}$ & $\begin{array}{l}\text { MGL } \\
\text { MGL }\end{array}$ & $\begin{array}{l}4.294 \\
4.160\end{array}$ & $\begin{array}{l}3.349 \\
3.349\end{array}$ & $\begin{array}{ll}10.36 & 3 \\
1.79 & 1\end{array}$ & $\begin{array}{l}0.002^{*} \\
0.322\end{array}$ \\
\hline
\end{tabular}

* Differences individually significant at the $5 \%$ level 


\section{Plot 1}

Page 6

Power curve for testing the difference in means between two samples assuming 4 data points in one sample and 2 data points in the second sample.

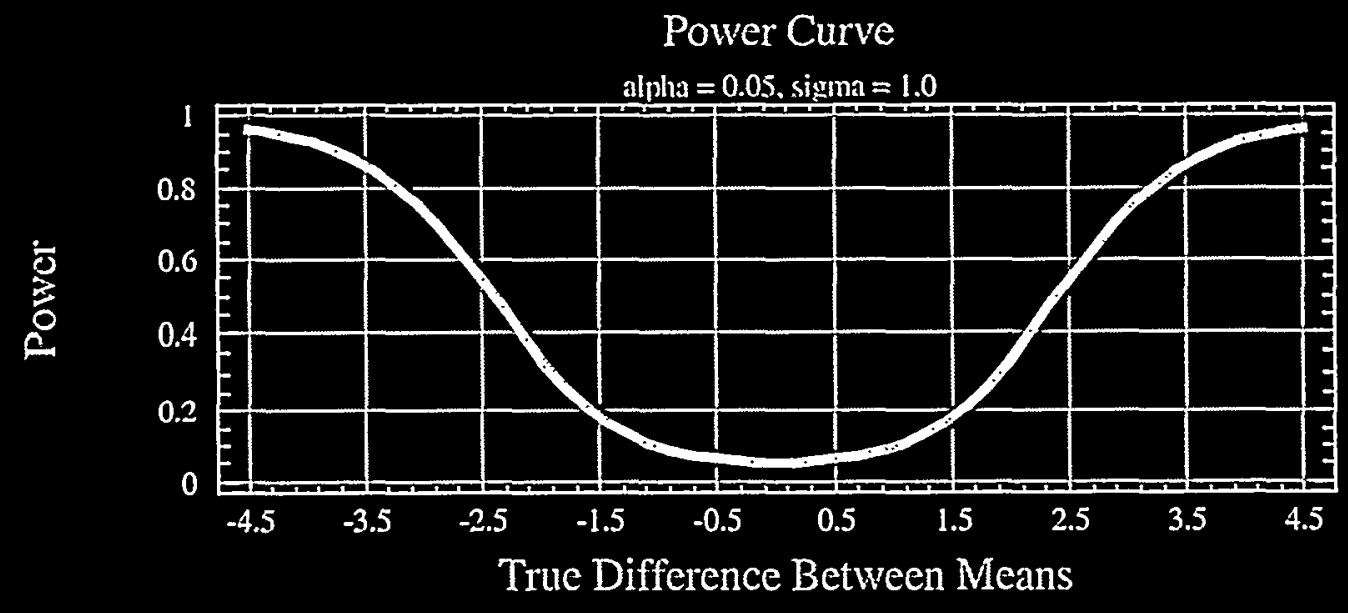




\section{Appendix 1}

Analyte Codes and Full Names

\begin{tabular}{|l|l|}
\hline Analyte & Full Name \\
\hline & \\
\hline ALPHAG & Gross alpha \\
\hline ALTOT & Aluminum total recoverable \\
\hline CATOT & Calcium total recoverable \\
\hline CCL4 & Carbon tetrachloride \\
\hline CL & Chloride \\
\hline COND & Specific conductance \\
\hline CRTOT & Chromium total recoverable \\
\hline FETOT & Iron total recoverable \\
\hline KTOT & Potassium total recoverable \\
\hline MNTOT & Manganese total recoverable \\
\hline NATOT & Sodium total recoverable \\
\hline PBTOT & Lead total recoverable \\
\hline FPH & Field pH \\
\hline TCLEE & Tetrachloroethylene \\
\hline TRCLE & Trichloroethylene \\
\hline
\end{tabular}


SRT-SCS-99-004

February 23, 1999

Page 8

Appendix 2

Average Results by Date Including Up To a Maximum of Four "Before" Sampling Days

Sdate: Sample Date

TYPE B: Before PWMS, A: After PWMS

Avg: Analyte Average

$\mathrm{N}$ : Number of results including below detects

PropD: Proportion of data above detect

\begin{tabular}{|c|c|c|c|c|c|c|c|}
\hline Well & Analyte & SDate & TYPE & Avg & Units & $\mathbf{N}$ & PropD \\
\hline ABP IA & ALPHAG & 7-Jul-94 & $B$ & 0.47225 & PCL & 2 & 0 \\
\hline ABP 1A & ALPHAG & 18-Jan-95 & $B$ & 0.808 & PCL & 1 & 1 \\
\hline ABP 1A & ALPHAG & 13-Mar-96 & $B$ & 0.711 & PCL & 1 & 1 \\
\hline ABP IA & ALPHAG & 3-Apr-96 & $B$ & 0.783 & $\mathrm{PCL}$ & 1 & 1 \\
\hline ABP 1A & ALPHAG & 11-Nov-97 & $A$ & 1.805 & PCL & 2 & 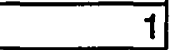 \\
\hline ABP 1A & ALPHAG & 13-Aug-98 & $A$ & 1.331083 & PCL & 5 & 0.4 \\
\hline ABP 1A & ALTOT & $7-J u l-94$ & $B$ & 0.01 & MGL & 1 & 0 \\
\hline ABP 1A & ALTOT & 18-Jan-95 & $B$ & 0.01665 & MGL & 1 & 을 \\
\hline ABP 1A & ALTOT & 13-Mar-96 & $B$ & 0.017325 & MGL & 2 & 0 \\
\hline ABP $1 \mathrm{~A}$ & ALTOT & 3-Apr-96 & $B$ & 0.125 & MGL & 1 & 0 \\
\hline ABP 1A & ALTOT & 11-Nov-97 & 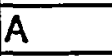 & 0.025 & MGL & 2 & 0 \\
\hline ABP 1A & ALTOT & 13-Aug-98 & $A$ & 0.026175 & MGL & 5 & 1 \\
\hline ABP 1A & CATOT & 13-Mar-96 & $B$ & 0.4345 & MGL & 2 & 1 \\
\hline ABP 1A & CATOT & 3-Apr-96 & $B$ & 0.748 & MGL & 1 & 1 \\
\hline ABP 1A & CATOT & 11-Nov-97 & $A$ & 0.5055 & MGL & 2 & 1 \\
\hline ABP 1A & CATOT & 13-Aug-98 & $A$ & 0.576583 & MGL & 5 & 1 \\
\hline ABP 1A & $\mathrm{CCL4}$ & $7-$ Jul-94 & $B$ & 0.0036 & MGL & 1 & 1 \\
\hline ABP 1A & CCL4 & 18-Jan-95 & $B$ & 0.00205 & MGL & 1 & 1 \\
\hline ABP 1A & CCL4 & 13-Mar-96 & $B$ & 0.00136 & MGL & 1 & 1 \\
\hline ABP 1A & CCL4 & 3-Apr-96 & $B$ & 0.00178 & MGL & 1 & 1 \\
\hline ABP 1A & $\mathrm{CCL} 4$ & 11-Nov-97 & $A$ & 0.000778 & MGL & 2 & 1 \\
\hline ABP 1A & $\overline{C C L 4}$ & 13-Aug-98 & $\bar{A}$ & 0.0015 & MGL & 4 & 0 \\
\hline ABP 1A & $\mathrm{CL}$ & 17-May-91 & $B$ & 3.73 & MGL & 1 & 1 \\
\hline ABP 1A & $\mathrm{CL}$ & 19-Jul-91 & $B$ & 1.615 & MGL & 2 & 1 \\
\hline ABP 1A & $\mathrm{CL}$ & 1-Nov-91 & $B$ & 1.52 & MGL & 1 & 1 \\
\hline ABP 1A & $\overline{C L}$ & 11-Jan-92 & $B$ & 1.55 & MGL & 1 & 1 \\
\hline ABP 1A & $\overline{C L}$ & 11-Nov-97 & A & 1.636667 & MGL & 3 & 1 \\
\hline ABP 1A & $\mathrm{CL}$ & 13-Aug-98 & $\mathrm{A}$ & 1.5775 & MGL & 5 & 1 \\
\hline ABP 1A & COND & 7-Jul-94 & $B$ & 13 & USCM & 1 & 1 \\
\hline ABP 1A & COND & 18-Jan-95 & $B$ & 12 & USCM & 1 & 1 \\
\hline ABP 1A & COND & 13-Mar-96 & $B$ & 16 & USCM & 1 & 1 \\
\hline ABP 1A & COND & 3-Apr-96 & $B$ & 12 & USCM & 1 & 1 \\
\hline ABP 1A & COND & 11-Nov-97 & $A$ & 14 & USCM & 2 & 1 \\
\hline ABP 1A & CRTOT & 18-Jan-95 & $B$ & 0.003335 & $M G L$ & 1 & 0 \\
\hline ABP $1 \mathrm{~A}$ & CRTOT & 13-Mar-96 & $B$ & 0.00541 & MGL & 2 & 1 \\
\hline ABP 1A & CRTOT & 3-Apr-96 & $B$ & 0.0125 & MGL & 1 & 0 \\
\hline ABP 1A & CRTOT & 11-Nov-97 & A & 0.0025 & MGL & 2 & 0 \\
\hline ABP 1A & CRTOT & 13-Aug-98 & $\mathrm{A}$ & 0.003273 & $\overline{M G L}$ & 5 & 0.6 \\
\hline ABP 1A & FETOT & 13-Mar-96 & $B$ & 0.07155 & MGL & 2 & 1 \\
\hline ABP 1A & FETOT & 3-Apr-96 & $B$ & 0.0149 & $M G L$ & 1 & 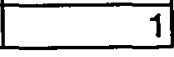 \\
\hline
\end{tabular}




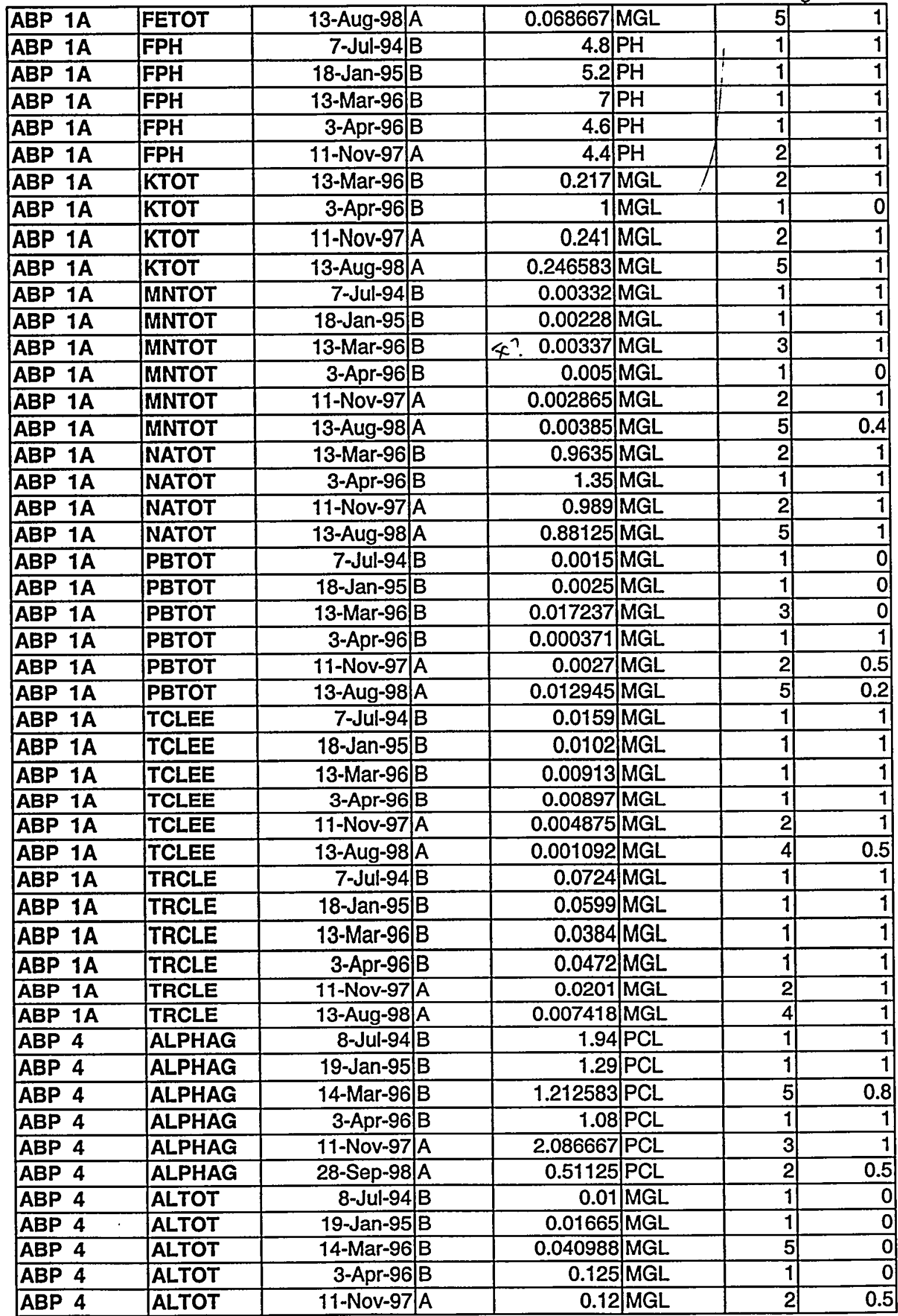


Page 10

\begin{tabular}{|c|c|c|c|c|c|c|c|}
\hline$\widehat{A B P} 4$ & ALTOT & 28-Sep-98|A & & 0.0162 & $\widehat{M G L}$ & 2 & 1 \\
\hline$\overline{A B P} 4$ & CATOT & 22-Jan-93 & $B$ & 0.610083 & MGL & 5 & 1 \\
\hline$\overline{A B P} 4$ & CATOT & 14-Mar-96 & $B$ & 0.474083 & $M G L$ & 5 & 1 \\
\hline \begin{tabular}{|l|l}
$A B P \quad 4$ \\
\end{tabular} & CATOT & 3-Apr-96 & $B$ & 0.557 & MGL & 1 & 1 \\
\hline \begin{tabular}{|l|l} 
ABP 4 \\
\end{tabular} & CATOT & 11-Nov-97 & $A$ & 0.388 & MGL & 2 & 1 \\
\hline$\overline{\mathrm{ABP}} 4$ & CATOT & 28-Sep-98 & $A$ & 0.571333 & MGL & 3 & 1 \\
\hline$\overline{A B P} 4$ & CCL4 & 8-Jul-94 E & $B$ & 0.0005 & $\overline{M G L}$ & 1 & 0 \\
\hline \begin{tabular}{|ll} 
ABP 4 \\
\end{tabular} & CCL4 & 19-Jan-95 & $B$ & 0.00219 & MGL & 1 & 1 \\
\hline $\begin{array}{l}\text { ABP } 4 \\
\end{array}$ & $\mathrm{CCL} 4$ & 14-Mar-96 & $B$ & 0.001684 & MGL & 5 & 1 \\
\hline 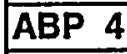 & CCL4 & 3-Apr-96 & $B$ & 0.0015 & MGL & 1 & 1 \\
\hline ABP 4 & $\mathrm{CCL} 4$ & 11-Nov-97 & $A$ & 0.00147 & MGL & 2 & 1 \\
\hline $\begin{array}{l}\text { ABP } 4 \\
\end{array}$ & CCL4 & 28-Sep-98 & $\mathrm{A}$ & 0.000523 & MGL & 2 & 1 \\
\hline$\overline{A B P \quad 4}$ & $\overline{\mathrm{CL}}$ & 19-Jul-91 & $B$ & 1.86 & MGL & 1 & 1 \\
\hline \begin{tabular}{|l|l}
$\mathrm{ABP}$ & 4 \\
\end{tabular} & $\mathrm{CL}$ & $1-$ Nov-91 & $B$ & 1.63 & MGL & 1 & 1 \\
\hline \begin{tabular}{|l}
$A B P$ \\
\end{tabular} & $\mathrm{CL}$ & 11-Jan-92 & $B$ & 1.72 & MGL & 1 & 1 \\
\hline \begin{tabular}{|l}
$\mathrm{ABP}$ \\
\end{tabular} & $\mathrm{CL}$ & 22-Jan-93 & $B$ & 1.393333 & $\mathrm{MGL}$ & 5 & 1 \\
\hline ABP 4 & $\mathrm{CL}$ & 11-Nov-97 & $A$ & 1.535 & $\overline{M G L}$ & 2 & 1 \\
\hline ABP 4 & $\mathrm{CL}$ & 28-Sep-98 & $A$ & 1.43 & $M G L$ & 3 & 1 \\
\hline$\overline{A B P \quad 4}$ & COND & 8-Jul-94 & $B$ & 14 & USCM & 1 & 1 \\
\hline ABP 4 & COND & 19-Jan-95 & $B$ & 11 & USCM & 1 & 1 \\
\hline ABP 4 & COND & 14-Mar-96 & $B$ & 14 & USCM & 1 & 1 \\
\hline$\overline{A B P} 4$ & COND & 3-Apr-96 & $B$ & 10 & USCM & 1 & 1 \\
\hline$\overline{A B P} 4$ & COND & 11-Nov-97 & $A$ & 19 & USCM & 2 & 1 \\
\hline ABP 4 & COND & 28-Sep-98 & $A$ & 17 & USCM & 2 & 1 \\
\hline \begin{tabular}{|l} 
ABP 4 \\
\end{tabular} & CRTOT & 22-Jan-93 & $B$ & 0.002 & MGL & 5 & 0 \\
\hline \begin{tabular}{|l} 
ABP 4 \\
\end{tabular} & CRTOT & 19-Jan-95 & $B$ & 0.003335 & $M G L$ & 1 & 0 \\
\hline \begin{tabular}{|l}
$\mathrm{ABP} \quad 4$ \\
\end{tabular} & CRTOT & 14-Mar-96 & $B$ & 0.002414 & MGL & 5 & 0.2 \\
\hline$\overline{A B P} 4$ & CRTOT & 3-Apr-96 & $B$ & 0.0125 & MGL & 1 & 0 \\
\hline ABP 4 & CRTOT & 11-Nov-97 & A & 0.00198 & MGL & 2 & 0.5 \\
\hline ABP 4 & CRTOT & 28-Sep-98 & $A$ & 0.00136 & MGL & 2 & 1 \\
\hline$\overline{A B P ~ 4}$ & FETOT & 22-Jan-93 & $B$ & 0.00425 & MGL & 5 & 0.4 \\
\hline ABP 4 & FETOT & 14-Mar-96 & $B$ & 0.0436 & MGL & 5 & 0.8 \\
\hline ABP 4 & FETOT & 3-Apr-96 & $B$ & 0.05 & MGL & 1 & 0 \\
\hline$\overline{A B P} 4$ & FETOT & 28-Sep-98 & $A$ & 0.1054 & MGL & 2 & 1 \\
\hline ABP 4 & FPH & 8-Jul-94 & $B$ & 4.8 & $\widehat{\mathrm{PH}}$ & 1 & 1 \\
\hline$\overline{A B P \quad 4}$ & FPH & 19-Jan-95 & $B$ & & 5 & 1 & 1 \\
\hline $\begin{array}{l}\text { ABP } 4 \\
\end{array}$ & FPH & 14-Mar-96 & $B$ & 4.6 & $\mathrm{PH}$ & 1 & 1 \\
\hline$\overline{A B P 4}$ & FPH & 3-Apr-96 & $B$ & 4.6 & $\mathrm{PH}$ & 1 & 1 \\
\hline$\overline{A B P \quad 4}$ & FPH & 11-Nov-97 & $A$ & 3.8 & $3 \mathrm{PH}$ & 2 & 1 \\
\hline ABP 4 & FPH & 28-Sep-98 & $\bar{A}$ & 5.7 & $\mathrm{PH}$ & 2 & 1 \\
\hline$\overline{A B P \quad 4}$ & KTOT & 22-Jan-93 & 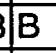 & 0.294833 & MGL & 5 & 0.2 \\
\hline$\overline{A B P} 4$ & KTOT & 14-Mar-96 & $B$ & 0.17975 & MGL & 5 & 1 \\
\hline ABP 4 & КTOT & 3-Apr-96 & $B$ & & $1 \mathrm{MGL}$ & 1 & 0 \\
\hline$\overline{A B P} 4$ & KTOT & 11-Nov-97 & $A$ & 0.1645 & \begin{tabular}{|l|l|} 
MGL \\
\end{tabular} & 2 & 1 \\
\hline$\overline{A B P} 4$ & КTOT & 28-Sep-98 & A & 0.191 & I MGL & 3 & 1 \\
\hline$\overline{A B P} 4$ & MNTOT & 8-Jul-94 & $B$ & 0.00233 & 3 MGL & 1 & 1 \\
\hline$\overline{A B P \quad 4}$ & MNTOT & 19-Jan-95 & $B$ & 0.00232 & MGL & 1 & 1 \\
\hline$\overline{A B P} 4$ & MNTOT & 14-Mar-96 & $B$ & 0.00251 & $1 \mathrm{MGL}$ & 5 & 1 \\
\hline$\overline{A B P} 4$ & MNTOT & 3-Apr-96 & $B$ & 0.005 & $5 \mid \mathrm{MGL}$ & 1 & 0 \\
\hline
\end{tabular}




\begin{tabular}{|c|c|c|c|c|c|}
\hline$\widehat{A B P} 4$ & MNTOT & 11-Nov-97|A & $0.018655 / M G L$ & 2 & 1 \\
\hline$\overline{A B P} 4$ & MNTOT & 28-Sep-98|A & $0.003695 / M G L$ & 2 & 1 \\
\hline ABP 4 & NATOT & 22-Jan-93 $\mathrm{B}$ & $0.8915 \mathrm{MGL}$ & 5 & 1 \\
\hline ABP 4 & NATOT & \begin{tabular}{l|l} 
14-Mar-96 & B
\end{tabular} & \begin{tabular}{l|l}
0.849583 & $M G L$ \\
\end{tabular} & 5 & 1 \\
\hline ABP 4 & NATOT & \begin{tabular}{l|l|}
$3-A p r-96$ & B \\
\end{tabular} & \begin{tabular}{l|l}
1.11 & $\mathrm{MGL}$ \\
\end{tabular} & 1 & 1 \\
\hline ABP 4 & NATOT & 11-Nov-97|A & \begin{tabular}{l|l|}
1.468 & MGL \\
\end{tabular} & 2 & 1 \\
\hline ABP 4 & NATOT & 28-Sep-98|A & $0.915 \mid \mathrm{MGL}$ & 3 & 1 \\
\hline ABP 4 & PBTOT & \begin{tabular}{|l|l|} 
8-Jul-94 & B \\
\end{tabular} & $\begin{array}{l}0.0015 \\
\end{array}$ & 1 & 0 \\
\hline ABP 4 & PBTOT & 19-Jan-95 B & $0.0025 \mathrm{MGL}$ & 1 & 0 \\
\hline ABP 4 & PBTOT & 14-Mar-96 $\mathrm{B}$ & $\begin{array}{ll}0.014063 \mathrm{MGL} \\
\end{array}$ & 5 & 0 \\
\hline ABP 4 & PBTOT & \begin{tabular}{|c|c|}
$3-A p r-96$ & B \\
\end{tabular} & \begin{tabular}{l|l|}
0.00162 & $M G L$ \\
\end{tabular} & 1 & 1 \\
\hline$\widehat{A B P} 4$ & PBTOT & \begin{tabular}{ll|}
$11-N o v-97$ & $A$ \\
\end{tabular} & $0.007755 \mathrm{MGL}$ & 2 & 1 \\
\hline ABP 4 & PBTOT & 28-Sep-98 $A$ & \begin{tabular}{l|l}
0.007032 & $M G L$ \\
\end{tabular} & 2 & 1 \\
\hline ABP 4 & TCLEE & \begin{tabular}{|l|l|}
$8-J u l-94$ & B \\
\end{tabular} & $\begin{array}{l}0.0005 \mathrm{MGL} \\
\end{array}$ & 1 & 0 \\
\hline ABP 4 & TCLEE & \begin{tabular}{l|l|} 
19-Jan-95 & $\mathrm{B}$ \\
\end{tabular} & \begin{tabular}{l|l}
0.0115 & $M G L$ \\
\end{tabular} & 1 & 1 \\
\hline$\overline{A B P} 4$ & TCLEE & 14-Mar-96 B & \begin{tabular}{l|l}
0.00833 & $M G L$ \\
\end{tabular} & 5 & 1 \\
\hline$\widehat{A B P ~} 4$ & TCLEE & \begin{tabular}{|c|c|}
$3-A p r-96$ & B \\
\end{tabular} & $0.00761 / \mathrm{MGL}$ & 1 & 1 \\
\hline$\overline{\text { ABP } 4}$ & TCLEE & $\begin{array}{ll}11-N o v-97 & A \\
\end{array}$ & \begin{tabular}{l|l|}
0.00642 & $M G L$ \\
\end{tabular} & 2 & 1 \\
\hline$\widehat{A B P 4}$ & TCLEE & 28-Sep-98 & 0.002645 MGL & 2 & 1 \\
\hline$\widehat{\text { ABP } 4}$ & TRCLE & \begin{tabular}{ll|l|}
$8-J u l-94$ & $B$ \\
\end{tabular} & \begin{tabular}{l|l}
0.0005 & $\mathrm{MGL}$ \\
\end{tabular} & 1 & 0 \\
\hline$\overline{\mathrm{ABP} 4}$ & TRCLE & 19-Jan-95B & $0.0834 \mathrm{MGL}$ & 1 & 1 \\
\hline ABP 4 & TRCLE & 14-Mar-96 & $0.050375 \mathrm{MGL}$ & 5 & 1 \\
\hline$\overline{\mathrm{ABP}} 4$ & TRCLE & \begin{tabular}{l|l|}
$3-A p r-96$ & $B$ \\
\end{tabular} & $0.0444 \mathrm{MGL}$ & 1 & 1 \\
\hline$\widehat{A B P 4}$ & TRCLE & \begin{tabular}{l|l}
$11-N o v-97$ & $A$
\end{tabular} & \begin{tabular}{l|l|}
0.0358 & $\mathrm{MGL}$ \\
\end{tabular} & 2 & 1 \\
\hline $\begin{array}{l}\text { ABP } 4 \\
\end{array}$ & TRCLE & 28-Sep-98 & \begin{tabular}{l|l}
0.0161 & MGL \\
\end{tabular} & 2 & 1 \\
\hline ARP 3 & ALPHAG & 20-Jan-93 B & $1 / P C L$ & 1 & 0 \\
\hline ARP 3 & ALPHAG & 10-Nov-95|B & $1.58 \mid \mathrm{PCL}$ & 1 & 1 \\
\hline ARP 3 & ALPHAG & 12-Dec-95 $\mathrm{B}$ & $2.285 \mathrm{PCL}$ & 2 & 1 \\
\hline ARP 3 & ALPHAG & \begin{tabular}{ll|} 
27-Feb-97 \\
\end{tabular} & \begin{tabular}{l|l}
1.72 & $\mathrm{PCL}$ \\
\end{tabular} & 1 & 1 \\
\hline ARP 3 & ALPHAG & \begin{tabular}{l|l} 
12-Nov-97 & $A$ \\
\end{tabular} & 3.93|PCL & 3 & 1 \\
\hline ARP 3 & ALPHAG & 13-Aug-98 $\mathrm{A}$ & $2.04325 \mid \mathrm{PCL}$ & 2 & 0.5 \\
\hline ARP 3 & ALTOT & \begin{tabular}{l|l}
$10-N o v-95$ & $B$ \\
\end{tabular} & 2.245 MGL & 2 & 1 \\
\hline ARP 3 & ALTOT & 12-Dec-95 & $0.0312 \mathrm{MGL}$ & 1 & 1 \\
\hline$\overline{\text { ARP } 3}$ & ALTOT & 15-Jan-96|B & 1.455 MGL & 2 & 1 \\
\hline ARP 3 & ALTOT & \begin{tabular}{l|l}
$27-F e b-97$ & $B$ \\
\end{tabular} & \begin{tabular}{l|l}
0.106 & $M G L$ \\
\end{tabular} & 1 & 1 \\
\hline ARP 3 & ALTOT & \begin{tabular}{l|l}
$12-N o v-97$ & $A$ \\
\end{tabular} & \begin{tabular}{l|l}
0.6804 & $M G L$ \\
\end{tabular} & 2 & 1 \\
\hline$\overline{\text { ARP } 3}$ & ALTOT & 13-Aug-98 A & 0.663 MGL & 2 & 1 \\
\hline ARP 3 & CATOT & 20-Jan-93 & 0.355 MGL & 1 & 1 \\
\hline ARP 3 & CATOT & 10-Nov-95 B & \begin{tabular}{l|l|l|}
0.483 & $M G L$ \\
\end{tabular} & 1 & 1 \\
\hline$\widehat{\text { ARP } 3}$ & CATOT & 12-Dec-95|B & $0.441 / \mathrm{MGL}$ & 1 & 1 \\
\hline ARP 3 & CATOT & 12-Nov-97 $A$ & $\begin{array}{l}0.3815 \mathrm{MGL} \\
\end{array}$ & 2 & 1 \\
\hline ARP 3 & CATOT & 13-Aug-98 $\mathrm{A}$ & \begin{tabular}{l|l}
0.5115 & $\mathrm{MGL}$ \\
\end{tabular} & 2 & 1 \\
\hline$\widehat{\text { ARP 3 }}$ & CCL4 & 10-Nov-95|B & $0.00025 \mathrm{MGL}$ & 1 & 0 \\
\hline ARP 3 & CCL4 & 12-Dec-95 B & $0.00025 \mathrm{MGL}$ & 1 & 0 \\
\hline ARP 3 & CCL4 & \begin{tabular}{l|l} 
15-Jan-96 & $\bar{B}$ \\
\end{tabular} & \begin{tabular}{l|l}
0.001 & $M G L$ \\
\end{tabular} & 1 & 0 \\
\hline$\overline{\text { ARP } 3}$ & CCL4 & 27-Feb-97|B & $0.0005 \mathrm{MGL}$ & 1 & 0 \\
\hline ARP 3 & CCL4 & 12-Nov-97|A & $0.00062 \mathrm{MGL}$ & 2 & 0.5 \\
\hline ARP 3 & CCL4 & 13-Aug-98 A & \begin{tabular}{l|l}
0.0005 & $M G L$ \\
\end{tabular} & 2 & 0 \\
\hline$\overline{\text { ARP } 3}$ & CL & 2-Apr-89|B & $7 \mid \mathrm{MGL}$ & 1 & 1 \\
\hline
\end{tabular}


SRT-SCS-99-004

February 23, 1999

Page 12

\begin{tabular}{|c|c|c|c|c|c|c|}
\hline ARP 3 & $\mathrm{CL}$ & 22-Jan-91|B & & \begin{tabular}{l|l|}
3.25 & $M G L$ \\
\end{tabular} & 1 & 1 \\
\hline ARP 3 & $\mathrm{CL}$ & 20-Jan-93 $\mid \bar{B}$ & $B$ & $2.54 \mathrm{MGL}$ & 1 & 1 \\
\hline \begin{tabular}{|l|} 
ARP 3 \\
\end{tabular} & $\mathrm{CL}$ & 27-Feb-97|B & $B$ & \begin{tabular}{l|l|}
2.45 & $M G L$ \\
\end{tabular} & 2 & 1 \\
\hline ARP 3 & $\mathbf{C L}$ & 12-Nov-97 $\mid \bar{A}$ & $A$ & \begin{tabular}{l|l}
2.6 & $\mathrm{MGL}$ \\
\end{tabular} & 3 & 1 \\
\hline \begin{tabular}{|l} 
ARP 3 \\
\end{tabular} & $\mathrm{CL}$ & 13-Aug-98/A & $A$ & 2.605 MGL & 2 & 1 \\
\hline ARP 3 & COND & 10-Nov-95 & $B$ & 20 USCM & 1 & 1 \\
\hline ARP 3 & COND & 12-Dec-95|B & $B$ & \begin{tabular}{l|l}
19 & USCM \\
\end{tabular} & 1 & 1 \\
\hline ARP 3 & COND & 15-Jan-96 & $B$ & 27USCM & 1 & 1 \\
\hline ARP 3 & COND & 27-Feb-97|B & $B$ & 18 USCM & 1 & 1 \\
\hline ARP 3 & COND & 12-Nov-97 & $\mathrm{A}$ & \begin{tabular}{|l|l|}
22 & USCM \\
\end{tabular} & 2 & 1 \\
\hline ARP 3 & CRTOT & 20-Jan-93 B & $B$ & \begin{tabular}{l|l|l}
0.002 & $M G L$ \\
\end{tabular} & 1 & 0 \\
\hline ARP 3 & CRTOT & 10-Nov-95 & $\mathrm{B}$ & \begin{tabular}{l|l|}
0.0548 & MGL \\
\end{tabular} & 2 & 1 \\
\hline ARP 3 & CRTOT & 12-Dec-95 E & $\mathrm{B}$ & $\begin{array}{l}0.00151 \\
\end{array}$ & 1 & 0 \\
\hline ARP 3 & CRTOT & 27-Feb-97|E & $B$ & $\begin{array}{l}0.0197 \\
\end{array}$ & 1 & 1 \\
\hline ARP 3 & CRTOT & 12-Nov-97 & $A$ & $0.008825 \mathrm{MGL}$ & 2 & 1 \\
\hline ARP 3 & CRTOT & 13-Aug-98 $A$ & $\mathrm{~A}$ & 0.013925 MGL & 2 & 1 \\
\hline ARP 3 & FETOT & 20-Jan-93 E & $B$ & \begin{tabular}{l|l}
0.0652 & $M G L$ \\
\end{tabular} & 1 & 1 \\
\hline ARP 3 & FETOT & 10-Nov-95 & $B$ & \begin{tabular}{r|r|}
7.92 & $\mathrm{MGL}$ \\
\end{tabular} & 2 & 1 \\
\hline ARP 3 & FETOT & 12-Dec-95 E & $B$ & $0.0847 \mathrm{MGL}$ & 1 & 1 \\
\hline ARP 3 & FETOT & 27-Feb-97 & $B$ & \begin{tabular}{l|l|l|}
0.586 & $M G L$ \\
\end{tabular} & 1 & 1 \\
\hline ARP 3 & FETOT & 13-Aug-98 & $A$ & \begin{tabular}{l|l|}
2.97 & $M G L$ \\
\end{tabular} & 2 & 1 \\
\hline ARP 3 & FPH & 10-Nov-95 & $B$ & \begin{tabular}{l|l|}
4.9 & $\mathrm{PH}$ \\
\end{tabular} & 1 & 1 \\
\hline ARP 3 & FPH & 12-Dec-95 E & $\mathrm{B}$ & \begin{tabular}{l|l|}
4.2 & $\mathrm{PH}$ \\
\end{tabular} & 1 & 1 \\
\hline ARP 3 & FPH & 15-Jan-96 E & $\mathrm{B}$ & \begin{tabular}{|l|l|}
4.6 & $\mathrm{PH}$ \\
\end{tabular} & 1 & 1 \\
\hline ARP 3 & FPH & 27-Feb-97|E & $\mathrm{B}$ & $4.4 \overline{\mathrm{PH}}$ & 1 & 1 \\
\hline ARP 3 & FPH & 12-Nov-97 & A & 4.1) $\mathrm{PH}$ & 2 & 1 \\
\hline ARP 3 & KTOT & 20-Jan-93 & $\mathrm{B}$ & \begin{tabular}{l|l}
0.25 & $\mathrm{MGL}$ \\
\end{tabular} & 1 & 0 \\
\hline ARP 3 & KTOT & 10-Nov-95 & $B$ & $\begin{array}{l}0.206 \\
\end{array}$ & 1 & 1 \\
\hline ARP 3 & KTOT & 12-Dec-95 & $B$ & $\begin{array}{l}0.149 \\
\end{array}$ & 1 & 1 \\
\hline ARP 3 & KTOT & 12-Nov-97 & $A$ & \begin{tabular}{|l|l|}
0.197 & $M G L$ \\
\end{tabular} & 2 & 1 \\
\hline ARP 3 & KTOT & 13-Aug-98 & $A$ & \begin{tabular}{l|l}
0.2025 & $\mathrm{MGL}$ \\
\end{tabular} & 2 & 1 \\
\hline ARP 3 & MNTOT & 10-Nov-95 & $B$ & \begin{tabular}{l|l}
0.0405 & $M G L$ \\
\end{tabular} & 1 & 1 \\
\hline \begin{tabular}{|l} 
ARP 3 \\
\end{tabular} & MNTOT & 12-Dec-95 & $\mathrm{B}$ & \begin{tabular}{l|l|}
0.00532 & $\mathrm{MGL}$ \\
\end{tabular} & 1 & 1 \\
\hline ARP 3 & MNTOT & 15-Jan-96 & $B$ & $0.0243 \mathrm{MGL}$ & 1 & 1 \\
\hline ARP 3 & MNTOT & 27-Feb-97 & $B$ & $0.0084 \mathrm{MGL}$ & 1 & 1 \\
\hline ARP 3 & MNTOT & 12-Nov-97 & $A$ & $0.021395 \mathrm{MGL}$ & 2 & 1 \\
\hline ARP 3 & MNTOT & 13-Aug-98 & $A$ & $\begin{array}{l}0.02532 \mathrm{MGL} \\
\end{array}$ & 2 & 1 \\
\hline ARP 3 & NATOT & 20-Jan-93 & $B$ & \begin{tabular}{l|l}
1.97 & $M G L$ \\
\end{tabular} & 1 & 1 \\
\hline ARP 3 & NATOT & 10-Nov-95 & $B$ & 1.79|MGL & 1 & 1 \\
\hline ARP 3 & NATOT & 12-Dec-95 & $\mathrm{B}$ & 1.77/MGL & 1 & 1 \\
\hline ARP 3 & NATOT & 12-Nov-97 & A & \begin{tabular}{l|l|l|}
2.015 & $M G L$ \\
\end{tabular} & 2 & 1 \\
\hline ARP 3 & NATOT & 13-Aug-98 & $A$ & $\begin{array}{l}2.505 \\
M G L \\
\end{array}$ & 2 & 1 \\
\hline ARP 3 & PBTOT & 10-Nov-95 & $B$ & $0.00245 \mathrm{MGL}$ & 1 & 1 \\
\hline ARP 3 & PBTOT & 12-Dec-95 & $\bar{B}$ & $0.00118 \mathrm{MGL}$ & 1 & 1 \\
\hline \begin{tabular}{|l} 
ARP 3 \\
\end{tabular} & PBTOT & 15-Jan-96 & $\mathrm{B}$ & $0.018037 \mathrm{MGL}$ & 3 & 0.6667 \\
\hline \begin{tabular}{|l} 
ARP 3 \\
\end{tabular} & PBTOT & 27-Feb-97 & $B$ & \begin{tabular}{l|l|}
0.0025 & $M G L$ \\
\end{tabular} & 1 & 0 \\
\hline ARP 3 & PBTOT & 12-Nov-97 & $A$ & \begin{tabular}{l|l}
0.0137 & $\mathrm{MGL}$ \\
\end{tabular} & 2 & 0.5 \\
\hline ARP 3 & PBTOT & 13-Aug-98 & $A$ & $0.0125 \mathrm{MGL}$ & 2 & 0.5 \\
\hline ARP 3 & TCLEE & 10-Nov-95 & $B$ & \begin{tabular}{l|l}
0.0091 & $M G L$ \\
\end{tabular} & 11 & 1 \\
\hline
\end{tabular}


Page 13

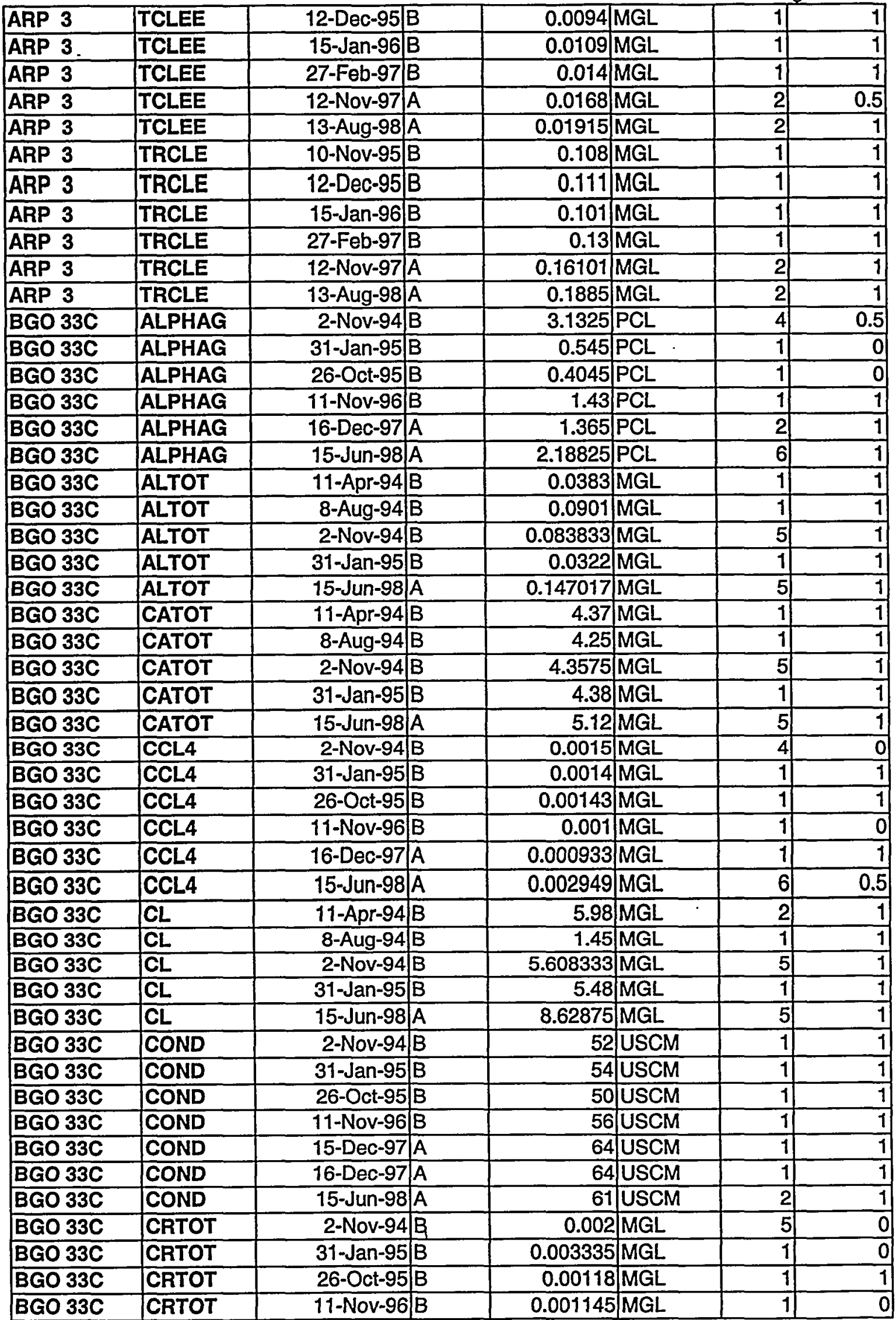


SRT-SCS-99-004

February 23, 1999

Page 14

\begin{tabular}{|c|c|c|c|c|c|}
\hline BGO 33C & CRTOT & 16-Dec-97 A & $0.00293 \mathrm{MGL}$ & 1 & 1 \\
\hline BGO 33C & CRTOT & 15-Jun-98|A & $0.00275 \mathrm{MGL}$ & 5 & 1 \\
\hline BGO 33C & FETOT & 11-Apr-94 $\mathrm{B}$ & \begin{tabular}{l|l|}
0.00637 & $M G L$ \\
\end{tabular} & 1 & 1 \\
\hline BGO 33C & FETOT & \begin{tabular}{|c|c|} 
8-Aug-94 & B \\
\end{tabular} & $0.0173 \mathrm{MGL}$ & 1 & 1 \\
\hline BGO 33C & FETOT & 2-Nov-94 B & \begin{tabular}{l|l}
0.016 & $M G L$ \\
\end{tabular} & 5 & 0.6 \\
\hline BGO 33C & FETOT & 31-Jan-95 $\mathrm{B}$ & $\begin{array}{l}0.00596 \mathrm{MGL} \\
\end{array}$ & 1 & 1 \\
\hline BGO 33C & FETOT & 15-Jun-98 $\mathrm{A}$ & $0.26935 \mathrm{MGL}$ & 5 & 1 \\
\hline BGO 33C & FPH & \begin{tabular}{l|l|} 
2-Nov-94 & B \\
\end{tabular} & $5.6 \mid \mathrm{PH}$ & 1 & 1 \\
\hline BGO 33C & FPH & \begin{tabular}{ll|l}
$31-J a n-95$ & $B$ \\
\end{tabular} & 5.6 $\mathrm{PH}$ & 1 & 1 \\
\hline BGO 33C & FPH & 26-Oct-95 B & \begin{tabular}{l|l|}
5.3 & $\mathrm{PH}$ \\
\end{tabular} & 1 & 1 \\
\hline BGO 33C & FPH & \begin{tabular}{l|l}
$11-N o v-96$ & $\mathrm{~B}$ \\
\end{tabular} & \begin{tabular}{l|l|l|}
5.4 & $\mathrm{PH}$ \\
\end{tabular} & 1 & 1 \\
\hline BGO 33C & FPH & 15-Dec-97 $A$ & \begin{tabular}{l|l|}
5.4 & $\mathrm{PH}$ \\
\end{tabular} & 1 & 1 \\
\hline BGO 33C & FPH & \begin{tabular}{l|l} 
16-Dec-97 & $\mathrm{A}$ \\
\end{tabular} & $5.4 \overline{\mathrm{PH}}$ & 1 & 1 \\
\hline BGO 33C & FPH & 15-Jun-98 A & \begin{tabular}{l|l|l}
5.2 & $\mathrm{PH}$ \\
\end{tabular} & 2 & 1 \\
\hline BGO 33C & KTOT & \begin{tabular}{l|l|}
$11-A p r-94$ & $B$ \\
\end{tabular} & \begin{tabular}{l|l|}
0.25 & MGL \\
\end{tabular} & 1 & 0 \\
\hline BGO 33C & KTOT & $\begin{array}{l}\text { 8-Aug-94 } \\
\end{array}$ & $0.596 \mid \mathrm{MGL}$ & 1 & 1 \\
\hline BGO 33C & KTOT & 2-Nov-94|B & $0.32075 \mathrm{MGL}$ & 5 & 0.2 \\
\hline BGO 33C & KTOT & \begin{tabular}{l|l|}
$31-J a n-95$ & $B$ \\
\end{tabular} & $0.505 \mathrm{MGL}$ & 1 & 1 \\
\hline BGO 33C & KTOT & 15-Jun-98 A & \begin{tabular}{l|l|l|}
1.737167 & $\mathrm{MGL}$ \\
\end{tabular} & 5 & 1 \\
\hline BGO 33C & MNTOT & 11-Apr-94 & $0.0302 \mathrm{MGL}$ & 1 & 1 \\
\hline BGO 33C & MNTOT & \begin{tabular}{l|l|}
$8-A u g-94$ & $B$ \\
\end{tabular} & $\begin{array}{l}0.0321 \\
\end{array}$ & 1 & 1 \\
\hline BGO 33C & MNTOT & \begin{tabular}{l|l|}
$2-N o v-94$ & $B$ \\
\end{tabular} & \begin{tabular}{l|l|}
0.03235 & $\mathrm{MGL}$ \\
\end{tabular} & 5 & 1 \\
\hline BGO 33C & MNTOT & 31-Jan-95 $\mathrm{B}$ & \begin{tabular}{l|l|}
0.034 & $\mathrm{MGL}$ \\
\end{tabular} & 1 & 1 \\
\hline BGO 33C & MNTOT & \begin{tabular}{l|l}
$15-J u n-98$ & $A$
\end{tabular} & \begin{tabular}{l|l|}
0.050258 & $\mathrm{MGL}$ \\
\end{tabular} & 5 & 1 \\
\hline BGO 33C & NATOT & 11-Apr-94 & 3.28 $\mathrm{MGL}$ & 1 & 1 \\
\hline BGO 33C & NATOT & 8-Aug-94|B & \begin{tabular}{l|l|l|}
3.28 & $\mathrm{MGL}$ \\
\end{tabular} & 1 & 1 \\
\hline BGO 33C & NATOT & 2-Nov-94 B & 3.395833 MGL & 5 & 1 \\
\hline BGO 33C & NATOT & \begin{tabular}{l|l|}
$31-J a n-95$ & $\mathrm{~B}$ \\
\end{tabular} & \begin{tabular}{l|l|l|}
3.44 & $M G L$ \\
\end{tabular} & 1 & 1 \\
\hline BGO 33C & NATOT & 15-Jun-98 $A$ & 4.294167 $\mathrm{MGL}$ & 5 & 1 \\
\hline BGO 33C & PBTOT & \begin{tabular}{|l|l|} 
2-Nov-94 & B \\
\end{tabular} & $\begin{array}{ll}0.0015 & M G L \\
\end{array}$ & 5 & 0 \\
\hline BGO 33C & PBTOT & 31-Jan-95|B & \begin{tabular}{l|l}
0.0025 & $M G L$ \\
\end{tabular} & 2 & 0 \\
\hline BGO 33C & PBTOT & \begin{tabular}{l|l|}
$26-O c t-95$ & $B$ \\
\end{tabular} & \begin{tabular}{l|l|}
0.0025 & $M G L$ \\
\end{tabular} & 1 & 0 \\
\hline BGO 33C & PBTOT & 11-Nov-96 B & \begin{tabular}{l|l|}
0.00127 & $M G L$ \\
\end{tabular} & 1 & 0 \\
\hline BGO 33C & PBTOT & 16-Dec-97|A & $0.0025 \mathrm{MGL}$ & 1 & 0 \\
\hline BGO 33C & PBTOT & 15-Jun-98 $A$ & $0.00987 \mathrm{MGL}$ & 5 & 1 \\
\hline$B G O 33 C$ & TCLEE & \begin{tabular}{l|l|} 
2-Nov-94 & B \\
\end{tabular} & \begin{tabular}{|l|l|}
0.00448 & MGL \\
\end{tabular} & 4 & 1 \\
\hline BGO 33C & TCLEE & \begin{tabular}{l|l}
$31-J a n-95$ & $\mathrm{~B}$ \\
\end{tabular} & \begin{tabular}{l|l}
0.0057 & $\mathrm{MGL}$ \\
\end{tabular} & 1 & 1 \\
\hline BGO 33C & TCLEE & 26-Oct-95 $\mathrm{B}$ & $0.00534 / \mathrm{MGL}$ & 1 & 1 \\
\hline BGO 33C & TCLEE & \begin{tabular}{l|l}
$11-N o v-96$ & $B$ \\
\end{tabular} & \begin{tabular}{ll|l}
0.006 & $M G L$ \\
\end{tabular} & 1 & 1 \\
\hline BGO 33C & TCLEE & $\begin{array}{ll}\text { 16-Dec-97 } \mathrm{A} \\
\end{array}$ & \begin{tabular}{|c|c|}
0.00598 & $M G L$ \\
\end{tabular} & 1 & 1 \\
\hline BGO 33C & TCLEE & $\begin{array}{ll}15-J u n-98 & A \\
\end{array}$ & $0.006305 \mathrm{MGL}$ & 6 & 1 \\
\hline BGO 33C & TRCLE & 2-Nov-94 B & $0.02675 \mathrm{MGL}$ & 4 & 1 \\
\hline BGO 33C & TRCLE & 31-Jan-95 B & \begin{tabular}{l|l}
0.0292 & $\mathrm{MGL}$ \\
\end{tabular} & 1 & 1 \\
\hline BGO 33C & TRCLE & $\begin{array}{lll}26-0 c t-95 & \mathrm{~B} \\
\end{array}$ & $0.025 \mathrm{MGL}$ & 1 & 1 \\
\hline BGO 33C & TRCLE & \begin{tabular}{l|l}
$11-N o v-96$ & $\mathrm{~B}$ \\
\end{tabular} & \begin{tabular}{l|l}
0.0304 & $\mathrm{MGL}$ \\
\end{tabular} & 1 & 1 \\
\hline BGO 33C & TRCLE & 16-Dec-97|A & \begin{tabular}{l|l}
0.0541 & $\mathrm{MGL}$ \\
\end{tabular} & 1 & 1 \\
\hline BGO 33C & TRCLE & 15-Jun-98|A & \begin{tabular}{l|l|}
0.040242 & $\mathrm{MGL}$ \\
\end{tabular} & 5 & 1 \\
\hline
\end{tabular}




\section{Appendix 3}

Page 15

Well: BGO 33C, Influent Sample Results Only

S_Date: Sample Date

S_Time: Sample Time

Detect: $1=$ Above Detect, $0=$ Below Detect

\begin{tabular}{|l|l|l|r|r|r|l|r|}
\hline Stream & Well & Analyte & S_Date & S_Time & Result & Units & Detected \\
\hline influent & BGO 33C & CATOT & 22-Dec-98 & $10: 06$ AM & 2.97 & MGL & 1 \\
\hline influent & BGO 33C & CATOT & 15-Jun-98 & $10: 08$ AM & 5.28 & MGL & 1 \\
\hline influent & BGO 33C & COND & 15-Jun-98 & $10: 08$ AM & 62 & USCM & 1 \\
\hline influent & BGO 33C & FETOT & 22-Dec-98 & $10: 06$ AM & 0.05 & MGL & 0 \\
\hline influent & BGO 33C & FETOT & $15-J u n-98$ & $10: 08$ AM & 0.0321 & MGL & 1 \\
\hline influent & BGO 33C & KTOT & $22-D e c-98$ & $10: 06$ AM & 0.419 & MGL & 1 \\
\hline influent & BGO 33C & KTOT & $15-J u n-98$ & $10: 08$ AM & 0.538 & MGL & 1 \\
\hline influent & BGO 33C & MNTOT & 22-Dec-98 & $10: 06$ AM & 0.0454 & MGL & 1 \\
\hline influent & BGO 33C & MNTOT & 15-Jun-98 & $10: 08$ AM & 0.0453 & MGL & 1 \\
\hline influent & BGO 33C & NATOT & 22-Dec-98 & $10: 06$ AM & 3.71 & MGL & 1 \\
\hline influent & BGO 33C & NATOT & 15-Jun-98 & $10: 08$ AM & 4.61 & MGL & 1 \\
\hline
\end{tabular}

